\title{
Power Amplifier for Magnetic Resonance Imaging using Unconventional Cartesian Feedback Loop
}

\author{
Von der Fakultät für Ingenieurwissenschaften, \\ Abteilung Elektrotechnik und Informationstechnik \\ der Universität Duisburg-Essen \\ zur Erlangung des akademischen Grades
}

Doktor der Ingenieurwissenschaften (Dr.-Ing.)

genehmigte Dissertation

von

Ashraf Abuelhaija

aus

Amman, Jordanien

Gutachter: Prof. Dr.-Ing. Klaus Solbach

Gutachter: Prof. Dr.-Ing. Stefan van Waasen

Tag der mündlichen Prüfung: 08.06.2016 



\section{ACKNOWLEDGEMENTS}

Allah says in the Quran: "And my success is not but through Allah. Upon him I have relied, and to Him I return". (Chapter 11, Verse 88)

I would like to take advantage of this opportunity to express my gratitude to my supervisor Prof. Dr-Ing. Klaus Solbach. I appreciate all his endless support, the ideas he contributed and the time he invested throughout the preparation of my $\mathrm{PhD}$. It has been an honor for me to be one of his students.

I am also thankful to Prof. Dr-Ing. Stephan van Waasen for being my second supervisor.

I would like to thank all my colleagues in HFT institute at University of Duisburg-Essen for their academic and personal support.

I extend my heartfelt thanks to my family in Jordan for their love, sacrifice, patience, encouragement, support and sincere prayers. I am deeply indebted to them.

Last but not least, I would like to express my gratitude and appreciation to all my friends, and to those who supported me for their continuous encouragement and help throughout my research.

Ashraf Abuelhaija

Duisburg, Germany

June, 2016 



\section{ABSTRACT}

Ultra-high field Magnetic Resonance Imaging (MRI) scanners (3T and higher) provide improved performance compared to lower field MRI scanners but because of RF magnetic flux $\left(B_{1}\right)$ inhomogeneity inside the patient's body, Multi-channel parallel RF transmission has been developed to allow $B_{1}$ optimization by, e.g., the RF shimming technique which requires a good decoupling of the RF coil elements to suppress induction of currents by neighbor coils so that the RF coil currents can be adjusted independently. However, current variation in array coils occurs also due to load change, degrading the shimming performance and leading to mismatch between the RF coil and the feed cable.

In this thesis, a near-magnet power amplifier (PA) is proposed without circulator output which employs a new concept of coil current sensing to allow control of the coil current. This power amplifier uses an unconventional Cartesian feedback loop (FBL) to compensate for current variation in an array coil by controlling the output voltage of the power amplifier. The particular property of the FBL is its capability to generate dynamic loop gain which can improve error compensation at high power level and system stability at low power level. To be still effective at low power, the near-magnet power amplifier is designed to present an ultra-low output impedance to the balun of the coil which suppresses coil current induced by mutual coupling from neighbor coils.

The power amplifier circuit design and characterization are discussed in detail by simulation in addition to a detailed analysis of the ultra-low output impedance with respect to coil decoupling. The design of the FBL is described and the performance evaluation is presented by simulation for the coil loading effect, coupling effect and PA nonlinearity. Experimental prototypes of PA and FBL have been fabricated in an MRI research project and measured performance supports simulation results. 



\section{ZUSAMMENFASSUNG}

Ultrahochfeld Magnetresonanztomographiegeräte (MRT, 3T und höher) bieten im Vergleich zu MRT-Geräten mit geringeren Feldstärken verbesserte Leistungen, erfordern jedoch aufgrund der in Körper der Patienten auftretenden $B_{1}$ Inhomogenität weitere Methoden der Optimierung. Hierfür wurde eine RF-Mehrkanal-Technik entwickelt, um die einzelnen RF-Spulen unabhängig voneinander aussteuern zu können. Allerdings wird diese Methode dadurch limitiert, dass Nachbarspulen Ströme induzieren können und durch Änderung der elektrischen Last ebenfalls eine Variation der Spulenimpedanz auftritt, die die Effizienz des Shimmings verringert und zu einer Fehlanpassung der RF-Spule führt.

Diese Arbeit beschreibt einen Leistungsverstärker der nahe am MR-Magneten angeordnet ist und daher ohne Zirkulator-Ausgang auskommen muss und der ein neues Konzept zur Abtastung der Spulenströme und somit zur Steuerung dieser bietet. Dieser Verstärker nutzt eine unkonventionelle kartesische Rückkopplungsschleife (FBL) zur Kompensation der Stromvariationen in einem Spulenelement durch Steuerung der Ausgangsspannung des Leistungsverstärkers. Die besondere Eigenschaft des FBL ist seine Möglichkeit, eine aussteuerungsabhängige Schleifenverstärkung zu generieren, die bei hoher Leistung die Fehlerkorrektur und bei niedriger Leistung die Systemstabilität verbessern kann. Um bei niedriger Leistung trotzdem effizient zu bleiben, wird der Leistungsverstärker so konzipiert, dass sein Ausgang eine ultra-niedrige Impedanz bezogen auf den Balun der Spulen darstellt. Dies unterdrückt den durch Verkopplung verursachten induzierten Strom in benachbarten Spulen.

Der Entwurf und die Charakterisierung der Verstärkerschaltung werden detailliert behandelt und mit Simulationen verdeutlicht, während außerdem eine detaillierte Analyse der ultra-niedrigen Ausgangsimpedanz im Zusammenhang mit der Spulenentkopplung durchgeführt wird. Der Entwurf der FBL wird beschrieben und eine Evaluation der Funktionsfähigkeit wird durch Simula- 
tionen zum Effekt der Lastimpedanz, der Spulen-Verkopplung und der Verstärker-Nichtlinearität durchgeführt. Experimentelle Prototypen des Verstärkers und des FBL wurden im Rahmen eines MRT-Forschungsprojekt hergestellt, so dass schließlich eine messtechnische Bestätigung der Simulationsergebnisse ermöglicht wurde. 


\section{CONTENTS}

List of Figures $\quad$ xiii

List of Tables $\quad$ xix

List of Acronyms $\quad$ xxi

1 INTRODUCTION

1.1 Background ............................ 1

1.2 Objectives of the Thesis . . . . . . . . . . . . . . . 3

1.3 Organization of the Thesis ................... 4

2 Magnetic Resonance Imaging System 7

2.1 Short History of MRI . . . . . . . . . . . . . . . . 7

2.2 MRI System Hardware $\ldots \ldots \ldots \ldots$

2.2 .1 Introduction . . . . . . . . . . . . . . . 8

2.2 .2 The Magnet . . . . . . . . . . . . . . . . . 8

2.2.3 The Gradient Coils . . . . . . . . . . . . . . . . . . . 9

2.2 .4 RF Coils . . . . . . . . . . . . . . . . . . 10

2.2 .5 RF Power Amplifiers . . . . . . . . . . . . . . . . . . . 10

$2.2 .6 \mathrm{~T} / \mathrm{R}$ Switch $\ldots \ldots \ldots \ldots \ldots \ldots$

2.3 Parallel RF Transmission . . . . . . . . . . . . . . . . . . . 11

2.4 High Q-factor MR Coils . . . . . . . . . . . . . . . . . . . . 14 
2.4.1 Loading Effect . . . . . . . . . . . . . . . . . . . 16

2.4 .2 Coupling Effect . . . . . . . . . . . . . . . . 17

2.4.3 Equivalent Circuit Model of Coupled Meander Coils . . . . . . . . . . 21

3 Conventional Rf Power Amplifiers for MrI 23

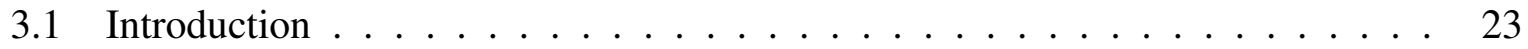

3.2 The Architecture of RFPAs for MRI . . . . . . . . . . . . . . . . 24

3.3 RFPA Classes . . . . . . . . . . . . . . . . . . . . . . . 24

3.4 Push-Pull Amplifier . . . . . . . . . . . . . . . . . . . 26

3.5 Specifications of RFPAs $\ldots \ldots \ldots \ldots \ldots \ldots$

3.5.1 Specifications in Time Domain . . . . . . . . . . . . . . . 31

3.5.2 Specifications in Frequency Domain . . . . . . . . . . . . . . 33

3.5.3 Specifications in Power Domain . . . . . . . . . . . . . . . 41

3.6 Near-Magnet Power Amplifier . . . . . . . . . . . . . . . . . . . . . . . . . 44

3.6 .1 Introduction . . . . . . . . . . . . . . . 44

3.6.2 Power Amplifier with Built-in Coil Current Sensing Concept . . . . . . 44

3.6.3 Design of a $1 \mathrm{~kW}$ power amplifier . . . . . . . . . . . 50

3.6.4 Characterization of the $1 \mathrm{~kW}$ power amplifier . . . . . . . . 58

3.6.5 Stability Analysis . . . . . . . . . . . . . . . 60

3.6.6 Output Impedance of the Power Amplifier . . . . . . . . . . . . . . . . 64

4 FEedBACK SYSTEM

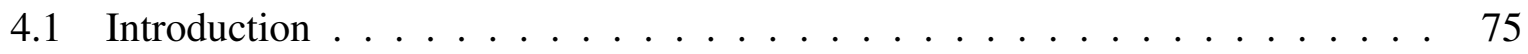

4.2 A brief history of Feedback . . . . . . . . . . . . 76

4.3 Basic Feedback System . . . . . . . . . . . . . . . . . . . . . . 76

4.4 Conventional Cartesian Feedback Loop . . . . . . . . . . . . . . . . . 80

5 Unconventional Cartesian FeEdback 83

5.1 Concept of Unconventional Cartesian Feedback Loop . . . . . . . . . . . . . . 83

5.2 System Architecture and Simulation Setup . . . . . . . . . . . . . . . . . . 84

5.3 Stability Analysis . . . . . . . . . . . . . . . . 85 
5.4 Experimental Prototype . . . . . . . . . . . . . . . . . . . . . . . . . . 89

5.5 Performance Evaluation of Feedback Loop . . . . . . . . . . . . . . . . . . . . 92

5.5.1 Loading Effect . . . . . . . . . . . . . . . . . . . . 92

5.5.2 Coupling Effect . . . . . . . . . . . . . . . . . . . 94

5.5 .3 Linearization . . . . . . . . . . . . . . . . 95

5.5.4 FBL Effect on Output Impedance . . . . . . . . . . . . . . . . . 96

6 Conclusion 99

A Appendix A: PA Prototype 101

B Appendix B: PA Characteristics $\quad 103$

C Appendix C: Experimental investigation of coil-PA interaction 105

$\begin{array}{ll}\text { Bibliography } & \mathbf{1 1 0}\end{array}$ 



\section{LIST OF FIGURES}

2.1 Block diagram of a MRI scanner. . . . . . . . . . . . . . . . 9

2.2 TR switch with preamplifier for $7 \mathrm{~T}$ system $(81.5 \mathrm{~mm} \times 42.5 \mathrm{~mm})$. . . . . . 11

2.3 TR switch using double face MSL hybrid couplers for 7T system (62 $\mathrm{mm} \times$ $62 \mathrm{~mm}$ ). (a) The $1 \mathrm{st}$ face, (b) the 2 nd face. . . . . . . . . . . . . . . . . 11

2.4 Quadrature excitation for RF body coil. . . . . . . . . . . . . . . 12

2.5 Two-channel RF shimming system. . . . . . . . . . . . . . . . . . 12

2.6 Static RF shimming. . . . . . . . . . . . . . . . . . 13

2.7 Dynamic RF shimming. . . . . . . . . . . . . . . . . . . . . 14

2.8 (a) MSL element, (b) Meander element, (c) Meander element with dielectric. 15

2.9 Simulated $|H|$ fields in a mid-sagittal section for (a) MSL element, (b) Meander element, (c) Meander element with dielectric. . . . . . . . . . . . . . . 15

2.10 Simulated $\left|B_{1}^{+}\right|$fields in a mid-transversal section for (a) MSL element, (b) Meander element, (c) Meander element with dielectric. . . . . . . . . . . . . 16

2.11 Simulation setup in HFSS software for single meander coil with a homogeneous phantom above the coil and RF shield below it . . . . . . . . . . . . . . 17

2.12 Demonstration of the loading effect. (a) Reflection coefficient $\left(S_{11}\right)$ vs. frequency as function of distance while the coil is matched at the Larmor frequency, (b) reflection coefficient $\left(S_{11}\right)$ vs. frequency as function of distance while the coil is matched at resonant frequency, (c) the corresponding reflection coefficient $(\Gamma)$ for the first case on the Smith chart, (d) the corresponding reflection coefficient ( $\Gamma)$ for the second case on the Smith chart. . . . . . . . . . . . . . . . . 18 
2.13 Series resonant circuit behaviour of meander coil. . . . . . . . . . . . . . 18

2.14 Simulation setup in HFSS software for two-coupled meander coils $\ldots \ldots$. . . . 19

2.15 T-equivalent network model for two coupled coil array elements. (a) Two active elements array, (b) one active element (left) and one matched terminated element

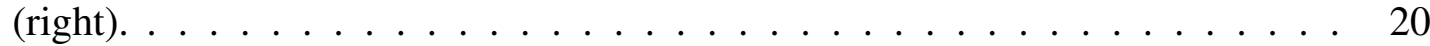

2.16 Reflection coefficient of a matched coil due to mutual coupling as function of power into the second coil. . . . . . . . . . . . . . . . 21

2.17 Equivalent circuit model of two-parallel meander coils. . . . . . . . . . . . . 22

$2.18 \mathrm{~S}_{11}$ and $\mathrm{S}_{21}$ based on ADS simulation and EM simulation. . . . . . . . 22

3.1 Block diagram of RFPA architecture. . . . . . . . . . . . . . . . . . 24

3.2 Operating points of an enhancement mode FET for different classes. . . . . . . 26

3.3 Block diagram of Push-Pull amplifier. . . . . . . . . . . . . . . . . . 27

3.4 Output characteristic with output wave forms for push-pull amplifier operating in class B mode. . . . . . . . . . . . . . . . . . . . . . . . . . . . 28

3.5 Output characteristic with output wave forms for push-pull amplifier operating in class $\mathrm{AB}$ mode. . . . . . . . . . . . . . . . . . . . . . . 29

3.6 (a) Push-pull amplifier using balanced center-tapped transformers, (b) ideal

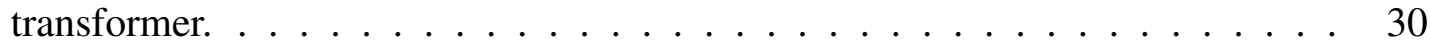

3.7 Ideal pulse waveform vs. actual pulse waveform. . . . . . . . . . . . 33

3.8 Second order nonlinearity. (a) Second order transfer characteristic, (b) time domain characteristic, (c) frequency domain characteristic. . . . . . . . . . . 36

3.9 Third order nonlinearity. (a) Third order transfer characteristic, (b) time domain characteristic, (c) frequency domain characteristic. . . . . . . . . . . . . . 38

3.10 Typical (AM/AM) and (AM/PM) curves. . . . . . . . . . . . . . . . 42

3.11 Representation of gain distortion and phase distortion in a given pulse sequence. 42

3.12 Circuit diagram for the new concept of power amplifier with built-in coil current sensing. . . . . . . . . . . . . . . . . . . 45

3.13 Voltage and current definitions for a lossless transmission line of length $l$, terminated by a load $Z_{2} \ldots \ldots \ldots \ldots \ldots \ldots$ 
3.14 The influence of attenuation $(\alpha=0.23 \mathrm{~dB} / \mathrm{m})$ in (multiple) half-wave cable on the ratio of the load voltage to the input voltage with load impedance $Z_{2}=25+\mathrm{j} \cdot 25 \Omega$. (a) Magnitude, (b) phase. . . . . . . . . . . . . . . . . . 50

3.15 Reflection coefficient calculated after lossless and lossy (multiple) half-wave cable in the range between $(\lambda / 2)$ and $100 *(\lambda / 2)$ and load impedance $Z_{2}=$ $25+\mathrm{j} \cdot 25 \Omega$.

3.16 Simulation setup in ADS software for 2-stage $1 \mathrm{~kW}$ power amplifier for 7T MRI. 52

3.17 Load pull simulation setup for the driver stage transistor (MRF6V2010). . . . . 52

3.18 Load pull simulation results for the MRF6V2010 transistor. (a) Region of load impedances covered by load tuner, (b) contours of equal power delivered with step size $0.5 \mathrm{~dB} \ldots \ldots \ldots \ldots \ldots$. . . . . . . . . . . . . . . . 53

3.19 Load pull simulation setup for the final stage transistor (BLF188XR). . . . . . 54

3.20 Load pull simulation results for BLF188XR transistor. (a) Region of load impedances covered by load tuner, (b) contours of equal power delivered with step size $2 \mathrm{~dB} \ldots \ldots \ldots \ldots \ldots \ldots \ldots$

3.21 (a) Symmetrical coaxial balun, (b) symmetrical balun equivalent circuit. . . . . 55

3.22 E-field pattern for balun coaxial cable. (a) First mode, (b) second mode. . . . . 56

3.23 The characteristics of the PA by different operating points. (a) Output power, (b) gain, (c) phase shift, (d) efficiency, (e) gain vs frequency at bias voltages $\mathrm{Dr}=3.3 \mathrm{~V}$ and $\mathrm{PA}=2 \mathrm{~V} \ldots \ldots \ldots \ldots \ldots$

3.24 Output power vs. load impedance. (a) At maximum drive power, (b) when driven for output power of $500 \mathrm{~W}$ with $50 \Omega$ load impedance. . . . . . . . . . 59

3.25 Transfer characteristic for fundamental, 2nd-order and 3rd-order signals of a single test signal. . . . . . . . . . . . . . . . . . . . 60

3.26 Transfer characteristic for fundamental and 3rd-order signals of a two test signals. 60

3.27 The Stability analysis technique utilizes a sinusoidal small-signal current source $i_{\text {in }}$ with frequency $f_{\mathrm{s}} \ldots \ldots \ldots \ldots \ldots \ldots$

3.28 Unstable region in the reflection coefficient plane for output power of $60 \mathrm{dBm}$ at $298 \mathrm{MHz}$ 
3.29 Simulated (circles) and identified (solid line) frequency response $H(\mathrm{j} \omega)$ for $\Gamma_{\mathrm{L}}=0.9 \angle 180^{\circ}$. (a) Magnitude, (b) phase, (c) associated pole-zero map $(\times$ :poles, $\bigcirc$ :zeros $) \ldots \ldots \ldots \ldots \ldots$

3.30 Simulated (circles) and identified (solid line) frequency response $H(\mathrm{j} \omega)$ for $\Gamma_{\mathrm{L}}=0.9 \angle 180^{\circ}$ with bandstop filters. (a) Magnitude, (b) phase, (c) associated pole-zero map $(\times$ :poles,$\bigcirc$ :zeros $) \ldots \ldots \ldots \ldots$

3.31 A schematic of a single receive element and preamplifier combination. . . . . 65

3.32 Ultra-low output impedance PA feeding a matched MR coil. . . . . . . . . . 65

3.33 The DC output characteristic for single transistor of BLF188XR . . . . . . . . 66

3.34 An output matching network in the last amplification stage. . . . . . . . . 66

3.35 Reflection coefficients for reference planes 1-4. . . . . . . . . . . . . 67

3.36 (a) Conventional transmit chain setup, (b) connection without circulator. . . . . 68

3.37 (a) Magnitude of output voltage for both setups under the sweep of the real reflection coefficient between -2 and 2, (b) simulated and measured output voltage at $500 \mathrm{~W}$ peak power (at $50 \Omega$ load) for direct connection setup under the sweep of the real reflection coefficient between -1 and $1 \ldots \ldots 68$

3.38 PA load dependence of (a) output power, (b) output voltage. . . . . . . . . . 69

3.39 Coupling measurement setup using two decoupled pick-up loops placed over a meander coil terminated by the PA. . . . . . . . . . . . . . . . . . 70

3.40 Measured coupling through coil with different coil terminations. . . . . . . . . 71

$3.41|H|$ in mid-transversal section for coupled meander coils as seen in FDTD simulator. (a) The second element is matched terminated, (b) the second element is terminated by the PA output impedance. . . . . . . . . . . . . . . . 71

3.42 Measurement setup for "hot S22" . . . . . . . . . . . . . . . . . 72

3.43 Simulated PA reflection coefficient $\left(\Gamma_{\mathrm{PA}}\right)$ as function of $(\mathrm{a})$ reverse power $(0 \mathrm{~W}$ $\longrightarrow 200 \mathrm{~W}),(\mathrm{b})$ forward power $(0 \mathrm{~W} \longrightarrow 1 \mathrm{~kW}) . \ldots \ldots 73$

3.44 The output reflection coefficient of HPA calculated after lossy (multiple) halfwave cable in the range between $0 \lambda$ and $100 \lambda \ldots \ldots$. . . . . . . . 74

4.1 Direct negative Feedback loop. . . . . . . . . . . . . . . . . . . . 77

4.2 Return ratio test circuit. . . . . . . . . . . . . . . . . . . 78 
4.3 Direct negative FBL with BPF. . . . . . . . . . . . . . . . . 78

4.4 Stability analysis diagrams for: direct negative Feedback loop (left) and direct negative Feedback loop with BPF (right). . . . . . . . . . . . . . . . 79

4.5 Conventional Cartesian Feedback loop with comparison at RF. . . . . . . . . . 81

4.6 Conventional Cartesian Feedback loop with extra forward path. . . . . . . . . . 81

5.1 Unconventional Cartesian Feedback loop. . . . . . . . . . . . . . . . . . . 83

5.2 Block diagram of simulation setup for one meander coil with unconventional Cartesian Feedback loop power amplifier. . . . . . . . . . . . . . . . . 85

5.3 Loop gain diagram of the open loop transfer function for different loop bandwidth. (a) Magnitude, (b) phase. . . . . . . . . . . . . . . . . . . . . 86

5.4 Stable (green color) and unstable (red color) regions for Pin $=0 \mathrm{dBm}$, Pout= $57 \mathrm{dBm}$ and loop bandwidth equal to (a) $1 \mathrm{MHz}$, (b) $500 \mathrm{kHz}$, (c) $100 \mathrm{kHz}$. . . 86

5.5 Simplified block diagram for unconventional Cartesian feedback loop. . . . . . 87

5.6 Dynamic loop gain as a function of input power for unconventional Cartesian feedback loop. (a) Magnitude, (b) phase. . . . . . . . . . . . . . . . 88

5.7 Loop gain as a function of input power for (a) unconventional Cartesian feedback loop, (b) conventional Cartesian feedback loop. . . . . . . . . . . . . . . . . . 88

5.8 Instability regions in the reflection coefficient plane $(-2 \leq \Gamma \leq 2)$ at $\mathrm{Pin}=0 \mathrm{dBm}$ (left), Pin $=-10 \mathrm{dBm}$ (middle) and Pin $=-20 \mathrm{dBm}$ (right) for (a) conventional Cartesian FBL, (b) unconventional Cartesian FBL. . . . . . . . . . . . . .

5.9 The prototype of our unconventional Cartesian feedback loop control unit including the pre-amplifier. . . . . . . . . . . . . . . . . . 91

5.10 Measured and simulated loop gain. (a) Magnitude, (b) phase. . . . . . . . . . . 91

5.11 Variation of power amplifier output voltage $V_{\text {out }}$ by sweeping phantom distance $d$. (a) When the coil is matched at Larmor frequency without FBL, (b) when the coil is matched at each new resonant frequency without FBL, (c) when the coil is matched at Larmor frequency with FBL, (d) when the coil is matched at each new resonant frequency with FBL . . . . . . . . . . . . . . . . . . 93

5.12 Normalized output voltage in complex domain for different coil loading situations. (a) When the coil is matched at Larmor frequency, (b) when the coil is matched at each new resonance frequency. 
5.13 Normalized output voltage in the complex domain for different coupling power in $\mathrm{dBm}$ when the phase of the second generator is set to (a) $0^{\circ}$, (b) $180^{\circ} \ldots 95$

5.14 Power amplifier transfer characteristic with and without feedback loop. (a) Magnitude in $\mathrm{dBm},(\mathrm{b})$ phase, $(\mathrm{c})$ gain. . . . . . . . . . . . . . . . . . . 96

5.15 Comparison of PA output voltage using the ULOI PA and using the ULOI PA in combination with the FBL at (a) Pin $=0 \mathrm{dBm}$, (b) Pin= $-10 \mathrm{dBm}$, (c) Pin= -20 $\mathrm{dBm},(\mathrm{d}) \mathrm{Pin}=-30 \mathrm{dBm}$

A.1 PA board with capacitor bank. . . . . . . . . . . . . . . . . . 101

A.2 High power amplifier board with the voltage probes $\left(U_{\mathrm{I}}\right.$ and $\left.U_{\mathrm{U}}\right)$ and $\lambda / 4$ transmission line in the output section. . . . . . . . . . . . . . . . . 102

B.1 Measured PA transfer characteristic . . . . . . . . . . . . . . . . . 103

B.2 Measured PA frequency response for a drive level in the linear region. . . . . 103

B.3 Measured PA output impedance as a function of frequency between $290 \mathrm{MHz}$

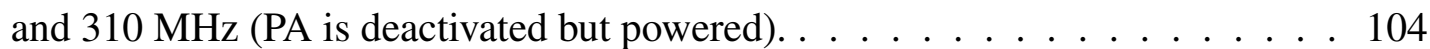

B.4 Measured third-order intercept due to intermodulation distortion of two test signals $200 \mathrm{kHz}$ apart. . . . . . . . . . . . . . . . . . . . 104

C.1 Measurement setup for coil reflection coefficient and coil current measurements. (a) Meander dipole coil approaches the phantom in steps, (b) meander coil seen from the back with balun and cable connections to the input and a voltage probe at the balun input $\left(\right.$ representing $\left.U_{\mathrm{I}}\right) \ldots \ldots \ldots$. . . . . . . . . . . 105

C.2 Magnetic current probe at meander dipole feed point . . . . . . . . . . 106

C.3 Demonstration of coil reflection coefficient variation due to coil loading with phantom distance varying from $1 \mathrm{~cm}$ to $20 \mathrm{~cm}$. . . . . . . . . . . . . . 106

C.4 Comparison of coil current variation due to coil loading using the magnetic current probe (blue) and the probe voltage $U_{\mathrm{I}}$ at the PA-Output (green). In red: $U_{\text {I with correction due to FBL } \ldots \ldots \ldots} \ldots \ldots$. . . . . . . . . . . . . 107 


\section{LIST OF TABLES}

3.1 Pulse parameter definitions. . . . . . . . . . . . . . . . . . 34

3.2 Isolation measured for different types of termination. . . . . . . . . . 70

5.1 Components of power amplifier and feedback loop. . . . . . . . . . . . . 91

5.2 Error $E_{\mathrm{R}}$ for output voltage magnitude and phase with and without feedback loop. 94

5.3 Error $E_{\mathrm{R}}$ for output voltage magnitude and phase due coupling effect with and without feedback loop. . . . . . . . . . . . . . . . . . . . . 95 



\section{LIST OF ACRONYMS}

\begin{tabular}{ll} 
ADC & Analog-to-Digital Converter \\
ADS & Advanced Design System \\
AM-AM & Amplitude Modulation-to-Amplitude Modulation \\
AM-PM & Amplitude Modulation-to-Phase Modulation \\
AMCAD & Advanced Modeling for Computer-Aided Design \\
BPF & Band Pass Filter \\
CAD & Computer-Aided Design \\
CW & Continuous Wave \\
DAC & Digital-to-Analog Converter \\
EM & Electromagnetic \\
FBL & Feedback Loop \\
FEM & Finite Element Method \\
fMRI & functional Magnetic Resonance Imaging \\
GBWP & Gain-Bandwidth Product \\
HB & Harmonic Balance \\
HFSS & High Frequency Structural Simulator \\
IMD & Intermodulation Distortion \\
IF & Intermediate Frequency \\
LDMOS & Laterally Diffused Metal Oxide Semiconductor \\
LO & Local Oscillator \\
MC & Microcontroller \\
MLSC & Microstrip Short-Circuited Stub \\
MRI & Magnetic Resonance Imaging \\
\hline
\end{tabular}


MSL Microstrip Line

NMR Nuclear Magnetic Resonance

OC Open Circuit

PA Power Amplifier

PTFE Polytetrafluoroethylene

RF Radio Frequency

RR Return Ratio

SC Short Circuit

SENSE SENSitivity Encoding

SNR Signal-to-Noise Ratio

T/R Transmit/Receive

TIAMO Time Interleaved Acquisition of Modes

TOI Third-Order Intercept

ULOI_PA Ultra Low Output Impedance Power Amplifier

VHF Very High Frequency 


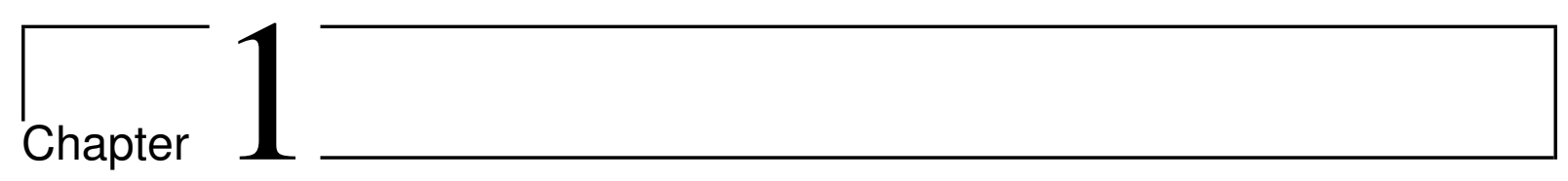

\section{INTRODUCTION}

\subsection{Background}

MRI scanners are considered useful devices for clinical diagnosis. They can produce highresolution images of any part in human body. Unlike computed tomography (CT) scanners and X-rays, MRI doesn't use ionizing radiation. This feature gives MRI scanners preference over other scanners because they don't cause harm to patients. In addition, MRI scanners have the capability for producing images in any of the three orthogonal planes (axial, coronal, and sagittal), as well as in oblique orientation without the need of the patient being scanned to move. Three-dimentional (3D) images of human organs are obtainable as well.

Medical imaging using the MRI technique relies heavily on the hydrogen atoms $(H)$ since the human body is mainly made up of water. Once the patient is placed inside the scanner, the hydrogen nuclei (protons) align parallel to the external magnetic field produced from the scanner magnet. RF coils in the scanner are responsible to excite the protons by sending circular polarized $(\mathrm{CP})$ radio frequency pulses of magnetic flux $\left(B_{1}^{+}\right)$at a frequency matching the resonant frequency of the protons. The excited protons in turn emit the signal back to the receive coils from where the signal is analysed and the image is created.

Since the early days of the medical use of MRI, rapid developments have been noted especially in the trend of the utilized magnetic field strength. MRI scanners employing higher magnetic field strength have proven the capability to increase the obtainable SNR which leads to higher image resolution. However, the higher the magnetic field strength being applied to the patient's body, the higher the excitation frequency is required. As a consequence, the wavelength of the exciting RF signal decreases and becomes comparable in size with the human organs. This 
decrement in wavelength causes strong RF interference, diffraction and attenuation effects which disturb the RF field distribution inside the human tissue by creating inhomogeneous RF transmit $\left(B_{1}^{+}\right)$field that might lead to signal voids in the field of view.

In a conventional single-channel RF system, one RF exciter is utilized to drive the body coil in quadrature to ensure the circular polarized excitation inside the human body. Once high magnetic field strength is used, it is desirable to increase the number of RF transmit channels: Parallel imaging that employs multi-transmit parallel RF transmission technology has been developed to overcome the inhomogeneity problem by, e.g., applying the RF shimming technique. This technology increases the degrees of freedom which are necessary to manipulate the RF excited signal in such away that a homogeneous excitation is obtained. In addition, parallel imaging has the capability to accelerate MR data acquisition, which in turn reduces the scan time.

An optimal RF shimming of the transmit $B_{1}$-field inside the human tissue requires a good isolation between the RF coil elements in order to drive the RF channels independently. Several decoupling methods have been proposed to eliminate strong electromagnetic coupling among the elements in coil arrays. In [1], a robust decoupling network has been implemented and inserted between the power amplifiers and the transmit array to achieve ideal decoupling. However, this concept shows only a good decoupling performance between the array element ports and cannot eliminate induced coil currents generated due to the mutual coupling when the coils have the characteristic of series resonance circuit. In [2], the overlap decoupling technique is used to decouple two adjacent loop-type coil elements in an array. However, overlap decoupling decreases parallel imaging performance by increasing the geometry (g-) factor in the overlapped area. Purely reactive decoupling methods have been presented to compensate for the mutual coupling between two adjacent coil elements [3,4]. All these decoupling techniques are focused on the elimination of the reactive component of the mutual impedance between the adjacent coil elements.

However, loading the MR coils by a sample which is specified as lossy dielectric (complex permitivity), makes the mutual impedance to contain a resistive component in addition to reactive component. The resistive component appears due to the conductivity of the sample which creates a common current paths between two coupled elements within the sample. In $[5,6]$ the concept of parasitic decoupling elements is proposed to compensate for both resistive and reactive components of the mutual impedance between two coupled RF coil elements. It generates magnetic flux equal in amount but oppositely oriented to the one generated by the passive element due to mutual coupling. 
Another solution for the coupling issue in transmit arrays has been proposed in [7]. The coils are driven through power amplifiers (PA), which behave as current source, so the current is impressed in the coil without deviation through induction by neighbouring coils. For that reason, the last stage transistor is matched in an unusual way such that the power amplifier output presents a very high impedance. Thus, the induced current is suppressed effectively. Such power amplifier can be realized by a high-voltage MOSFET to have high output impedance which enables current source operation over a wide loading range. It is assumed in this concept, that the coil can be represented as a series resonant circuit. Current-source power amplifiers are considered to be the best option in case isolation between array coils has the priority over the amount of power delivered.

In $[8,9]$, an ultra-low output impedance RF power amplifier for 3T and 7T MRI systems has been suggested, respectively. Through a suitable matching network between the coil and the last stage transistor, maximum power can be delivered to the matched load. Simultaneously, through an appropriate design for the output match of the power amplifier to present low impedance to the coil, the induced current in the coil highly reduces. This requires that the coil matching network is not only used to up-transform the low coil impedance to the transmission line impedance but also to present the coil as a parallel resonant circuit in order to improve the inter-coil isolation of a transmit array by letting the power amplifier impedance "short-circuit" the induced coil voltage.

Another approach is proposed with the use of a negative feedback (Cartesian Feedback) in the power amplifier [10,11], when the coil current is measured directly or indirectly and its deviation due to mutual coupling or load change is reduced by a closed loop compensation to a minimum. With this concept, the power amplifier can be used with the conventional match for optimum power and the coil matched to the line impedance. The use of Cartesian Feedback changes the over-all behavior of the power amplifier and lets it behave as current source. This creates a high impedance in series with the coil without loss of transmit efficiency. An additional massive study using Cartesian feedback loop amplifiers in order to reduce the electromagnetic coupling among the array elements has been done by $[12,13]$.

\subsection{Objectives of the Thesis}

This thesis introduces a new concept of a power amplifier for use in a near-magnet transmit array. The concept combines the use of an ultra-low output impedance power amplifier with a 
Cartesian feedback loop and an MR coil. The thesis tries to show how the output impedance of the power amplifier can suppress mutual coupling effects in the MR coil and how the feedback loop and the MR coil can be designed to compensate coil mismatch due to load variation and mutual coupling.

The thesis concentrates on the RF design aspects and relies on simulation, in most part based on the nonlinear transistor models supplied by the manufacturer of the active elements. In the research project ${ }^{1}$ leading to the effort described in this thesis a team at the department of High Frequency Technology, Duisburg-Essen University has designed, built and tested a 32channel transmit array using the results of the presented simulations and experimental prototypes. In the process, various modifications have been introduced into prototypes so that a one-toone verification of simulation and realized circuit has not been possible (and not required). Nevertheless, several results of realized amplifiers are presented in the appendix in order to support the simulation study.

\subsection{Organization of the Thesis}

This thesis consists of six chapters, including this introduction chapter. Chapter 2 provides background on MR system hardware and describes in detail the functionality of the main components that compose the MRI scanner. The conventional single-channel RF system as well as a multi-channel RF system are explained. A comparison between three recently developed high Q-factor MR coils in terms of $H$-field distribution and localized sensitivity is presented. In addition, coupling between the coils and mismatch of the loaded coils are considered a significant challenge for RF coil designers.

Chapter 3 talks about RF power amplifiers for MRI. A PA utilizing transistors in push-pull configuration is explained in detail showing their advantages. PAs used in MRI systems have certain specifications in time domain, frequency domain and power domain which are presented next. The concept of a near-magnet power amplifier with built-in coil current sensing is developed and verified theoretically. A prototype of such an ultra-low output impedance power amplifier utilizing the new current sensing concept is discussed in detail with PA circuit design and characterization by simulation and with a detailed analysis of the ultra-low output impedance with respect to coil decoupling. Some (non-complete) experimental verification of the amplifier

\footnotetext{
${ }^{1}$ MRexcite, ECR grant Agreement n.291903
} 
design is found in the appendix.

Chapter 4 studies the utilization of negative feedback loop concepts in combination with a PA in a system, starting from the direct negative feedback and introducing the conventional Cartesian feedback. The stability of the feedback systems and methods of test are of particular concern.

In chapter 5, the concept of a near-magnet PA with an unconventional Cartesian feedback is introduced and analyzed with respect to its stability and its ability to compensate for the output voltage variation due to mutual coupling and mismatch as well as capability to compensate for PA nonlinearity. The design of a prototype of an unconventional Cartesian feedback loop is described and its performance tested by simulation for coil loading effect, coupling effect and PA nonlinearity. Again, some experimental data are found in the appendix to verify the basic theoretical results. 



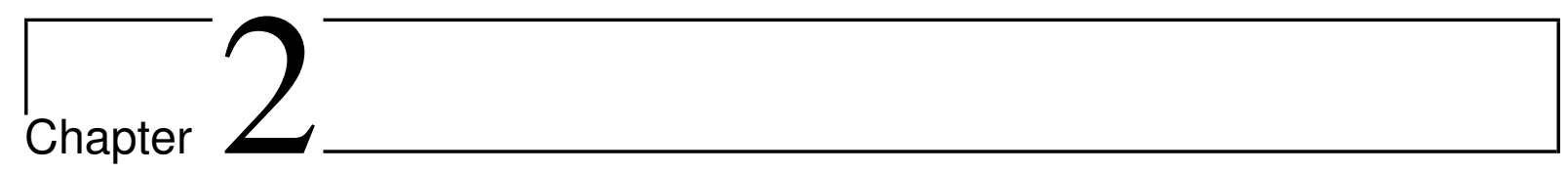

\section{MAGNetic Resonance IMAGing}

\section{SYSTEM}

\subsection{Short History of MRI}

When the history of MRI is studied, we should go back in the history and mention the mathematician and physicist Jean-Baptiste Joseph Fourier (1768-1830), who is known for his investigation of Fourier series and Fourier transform. And without Fourier transform, MR images could not be created. His mathematical transformation method developed for analysis of heat transfer [14] was first used for magnetic resonance signal analysis and image reconstruction by Richard Ernst in 1975.

In 1882, Nikola Tesla (1856-1943) discovered the rotating magnetic field [15]. Sir Joseph Larmor (1857-1942), who was the first to calculate the rate of energy radiation from an accelerating electron and the first to explain the splitting of the spectral lines in a magnetic field by the oscillation of electrons [16], became most famous in the MRI field due to the equation that holds his name-The Larmor equation. Isidor Rabi (1898-1988) studied the magnetic properties of atomic nuclei, and in 1938 he succeeded in determining the magnetic moments of the nuclei [17]. In 1946, Felix Bloch (1905-1983) and Edward Purcell (1912-1997) independently discovered the nuclear magnetic resonance (NMR) phenomenon $[18,19]$. They found that when nuclei are placed in a magnetic field, they absorb energy at a specific resonance frequency based on the Larmor equation. Nuclei re-emit this energy when they return to the original state. In 1950, Erwin L. Hahn discovered the spin-echo method [20]. In 1971, Raymond Damadian discovered the idea for detecting cancer in tissue by nuclear magnetic resonance [21]. He figured out that 
tumor tissue has longer relaxation times than normal tissue. In 1974, Paul C. Lauterbur and Peter Mansfield described, with their independent work, the use of magnetic field gradients for spatial localization of NMR signals. With this discovery, they put the foundation for Magnetic Resonance Imaging (MRI). In 1973, Paul Lauterbur produced the first 2D NMR images, while in 1977 Raymond Damadian produced MR image of the whole body. The first transverse NMR image through a human head was reported by Hugh Clow and Ian R. Young in 1978 [22]. In 1981, Hawkes and his colleagues produced the first NMR imaging of the heart [23].

In the early 1980's, the imaging technique of MRI had been introduced in clinical application using $0.15 \mathrm{~T}$ scanner.

The development of functional magnetic resonance imaging (fMRI) caused much excitement among researchers by the early 1990's. It is a technique used to obtain functional imaging for the study of the regions of the brain responsible for certain activities.

Nowadays, imaging by MR scanners has become routine in many hospitals and imaging centres to diagnose most ailments of the brain, musculoskeletal problems, most spinal conditions/injuries, female pelvic problems, prostate problems, and many other health problems or diseases.

\subsection{MRI System Hardware}

\subsubsection{Introduction}

MRI scanners are considered one of the best examples in medical application that employ RF technology. They benefits from the interaction between three different systems (magnet, gradient coils, and radio frequency system) to generate an MRI images. The radio frequency (RF) system is responsible to stimulate the nuclei of the body tissue by transmitting pulsed magnetic RF fields. Furthermore, it receives the MR signal that is induced by the stimulated nuclei. This system consists of RF transmitter and RF receiver. The RF transmitter contains a frequency synthesizer, the digital envelope modulator of RF frequencies, a high power amplifier, and an RF coil. The $\mathrm{RF}$ receiver contains an RF coil, a preamplifier, a filter, and analog-to-digital converter (ADC).

\subsubsection{The Magnet}

The magnet is considered the most expensive component in MRI scanners. It is responsible for generating a strong longitudinal static magnetic field, $B_{0}$ [Tesla], (directed along the $z$ axis). Most MRI scanners use superconducting magnets which consists of superconducting wires 


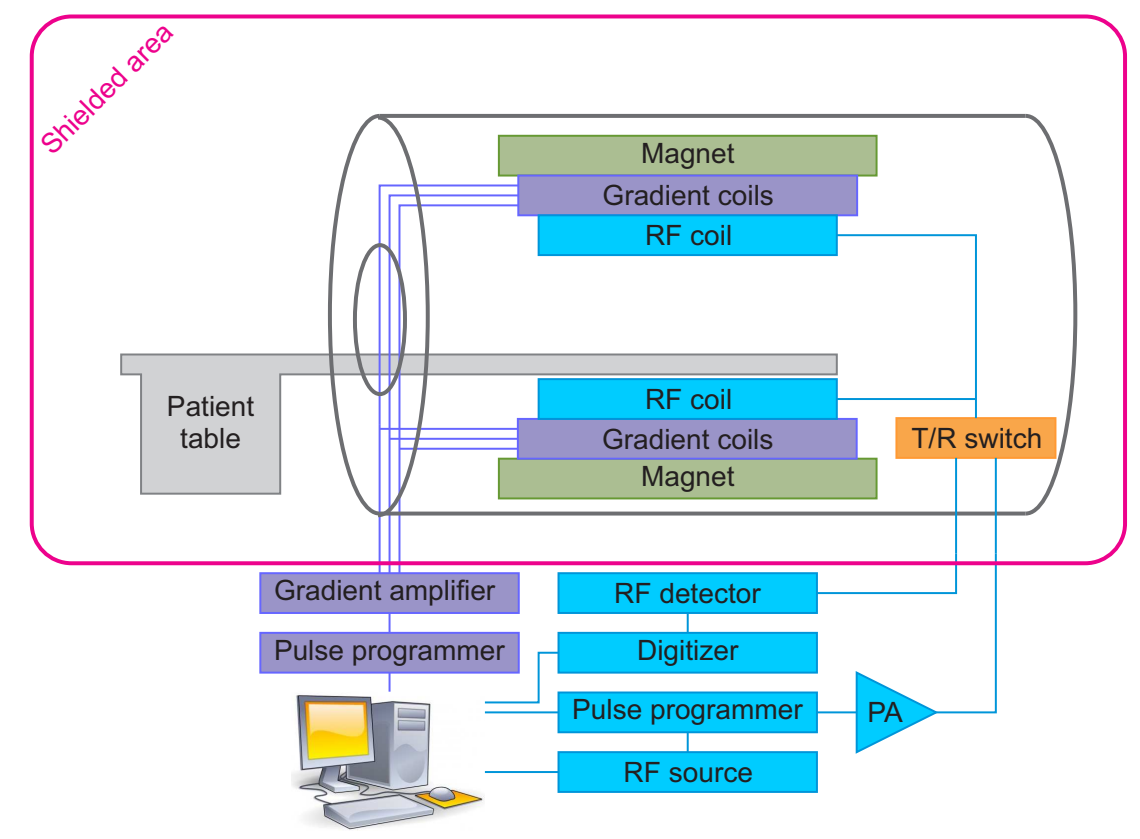

Figure 2.1: Block diagram of a MRI scanner.

driven by a large current, due to its capability to produce very strong and stable magnetic field. Maintaining such a large magnetic field requires a high reduction in the resistance of the superconducting wires to approach almost zero. This can be done by immersing it in liquid helium to cool it to a temperature close to absolute zero $\left(-273.15^{\circ} \mathrm{C}\right.$ or $\left.0 \mathrm{~K}\right)$.

When a subject is placed in the scanner, the hydrogen atoms (protons) either align with or against $B_{0}$ and precess at the Larmor frequency or precession frequency, $\omega_{0}[\mathrm{rad} / \mathrm{sec}]$. This frequency is linearly related to the main magnetic field $B_{0}$ and calculated from Larmor equation, $\omega_{0}=\gamma B_{0}$, where $\gamma$ is the gyromagnetic ratio or a constant depending on the nucleus.

\subsubsection{The Gradient Coils}

The gradient coils are a set of 3 magnets responsible for spatially-linear variations in the magnetic field in the three orthogonal directions $(x, y, z)$. This variation in the magnetic field permits localization of image slices without requiring the patient being scanned to move. Typical gradient systems are capable of producing gradients from 20-100 mT/m. During the imaging process, sounds or noises are produced due to the fast switching of the gradients. Rapid variation in magnetic field produces undesirable electric currents (eddy currents) in conducting materials throughout the scanner. The eddy currents create magnetic fields that can reduce the performance of gradient field as well as distort the images. 


\subsubsection{RF Coils}

Radio frequency coils (RF coils) are antennas responsible for transmitting RF pulses perpendicular to the main magnetic field $B_{0}$ in the transverse plane (x-y plane), and receiving the returned signals from the patient. During transmission phase, the radio frequency magnetic pulses of flux $B_{1}$ are tuned to the Larmor frequency to flip the protons by a certain flip angle from the steady state alignment (parallel to $B_{0}$ ) leading to an energy absorption by the protons. When the pulsed radio frequency transmission is stopped, the protons start to release the absorbed energy to return to the steady state. This energy will be then detected by an RF coil as MR signal to create the image.

In MRI, we can distinguish between two basic types of RF coils : Volume transmit coils or body coils and surface receive coils. For RF transmission, volume coils are desirable to use due to their capability to generate nearly homogeneous RF magnetic field over a large field of view. For MR signal reception, surface coils are desirable due to their high magnetic sensitivity obtained when placed directly over the region of interest. And because they extend over a small field of view, a better image quality is obtained due to the improvement in the signal to noise ratio (SNR) for the region of interest.

\subsubsection{RF Power Amplifiers}

MRI scanners, operating at high magnetic field strength not only increase the resonance frequency of the hydrogen atoms, but also increase the demand on RF power required to produce the RF pulses. The required RF power for excitation is proportional to the square of the magnetic field strength [24]. Therefor, at $1.5 \mathrm{~T}$ magnetic field strength, the RF power required is about nine times that needed at $0.5 \mathrm{~T}$ strength. To satisfy this requirement, RF high power amplifiers are placed at the end of the transmit chain in the system to drive the RF coils with suitable power level.

\subsubsection{T/R Switch}

The T/R switch is a major device in an MRI system whenever a single coil is used for transmit and receive (transceive). During transmit mode, it directs a high power RF signal from the RF amplifier toward the coil and protects the receive preamplifier. During receive mode, it directs the MR signal detected by the coil toward the receiver and isolates the transmitter. 
Fig. 2.2 shows a compact T/R switch has been produced in our lab based on Watkins' design [25]. It has low insertion loss of $0.5 \mathrm{~dB}$, and quite high preamplifier isolation during transmit of $+55 \mathrm{~dB}$. Fig. 2.3 shows our modified version that we have fabricated by two microstrip line (MSL) hybrid couplers printed on two Rogers corporation RO3010 substrates $\left(\varepsilon_{\mathrm{r}}=10.2\right.$, $\tan \delta=0.0022$ ) of $1.27 \mathrm{~mm}$ thickness. Their ground planes join each other to form a double face circuit. An internal connection between the couplers has been achieved by using thin wires passing through the ground planes. This version is larger but more robust than the first version in terms of heat dissipation when applying $1 \mathrm{~kW}$ peak power.

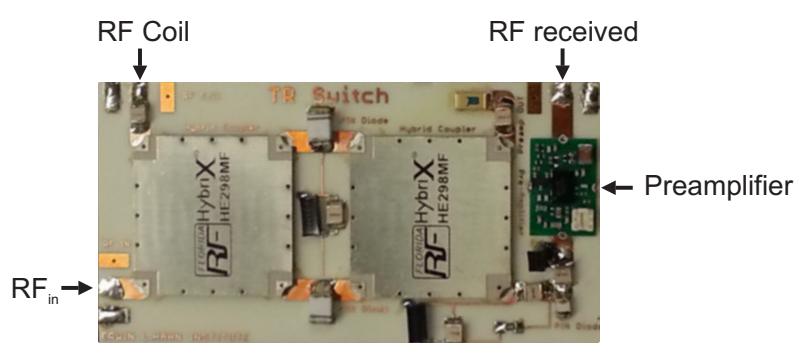

Figure 2.2: TR switch with preamplifier for $7 \mathrm{~T}$ system $(81.5 \mathrm{~mm} \times 42.5 \mathrm{~mm})$.

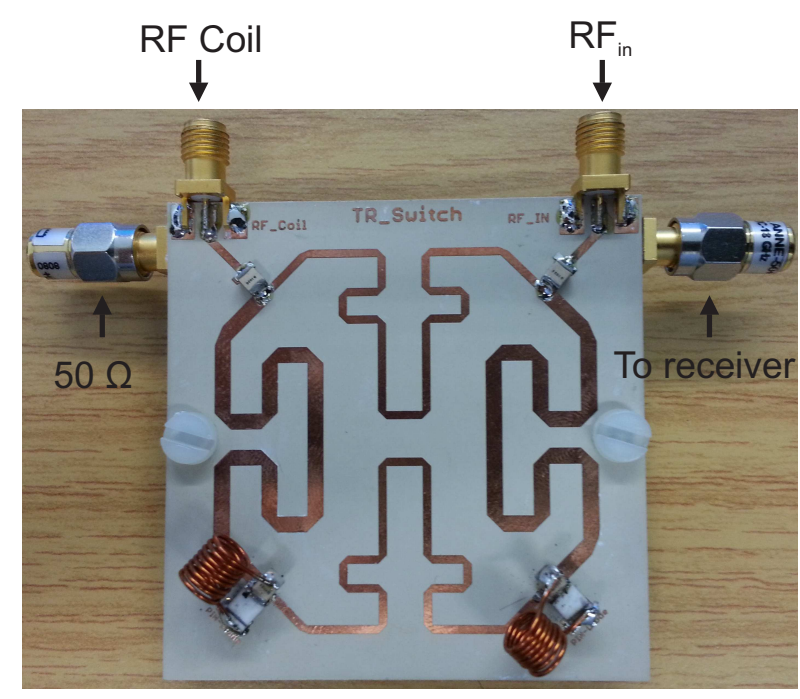

(a)

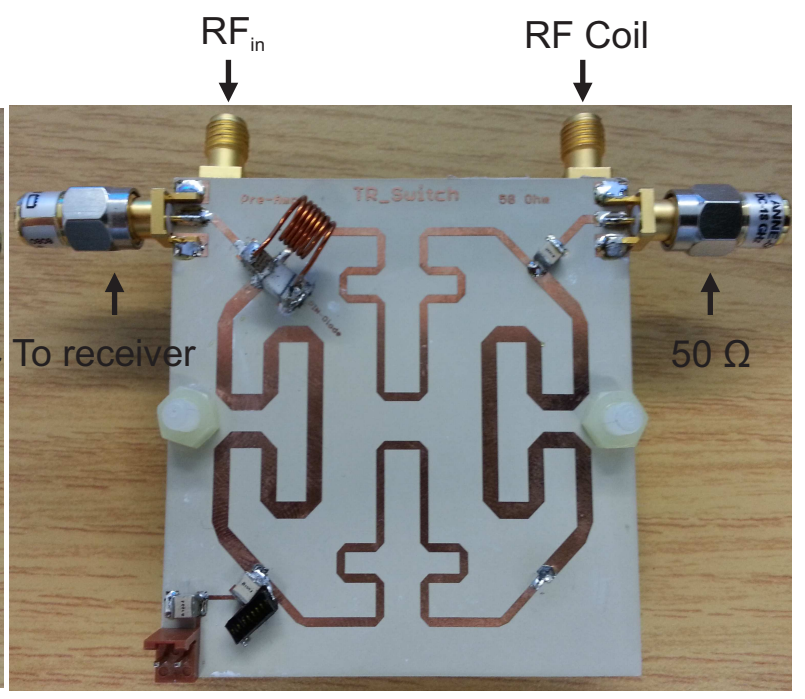

(b)

Figure 2.3: TR switch using double face MSL hybrid couplers for 7T system $(62 \mathrm{~mm} \times 62 \mathrm{~mm})$. (a) The 1st face, (b) the 2nd face.

\subsection{Parallel RF Transmission}

Ultra high magnetic field (7 $\mathrm{T}$ and higher) MRI scanners are superior over lower magnetic field MRI scanners in terms of obtainable signal to noise ratio (SNR) which scales linearly with the 
main magnetic field ( $\mathrm{SNR} \propto B_{0}$ ) [26]. Higher SNR allows to produce higher resolution images. However, also the Larmor frequency is proportional to the main magnetic field strength. This will decrease the wavelength of the exciting RF signal. With the dielectric property of a tissue, the wavelength inside this tissue can be determined by $\lambda_{\text {tissue }}=c /\left(\sqrt{\varepsilon_{\mathrm{r}}} \cdot f_{0}\right)$, where $c$ is the light velocity, $\varepsilon_{\mathrm{r}}$ is dielectric permittivity and $f_{0}$ is the Larmor frequency. At $7 \mathrm{~T}$, the wavelength inside the human body $\left(\varepsilon_{\mathrm{r}, @ 7 \mathrm{~T}} \approx 40\right)$ is approximately $15 \mathrm{~cm}$ and inside the head $\left(\varepsilon_{\mathrm{r}, @ 7 \mathrm{~T}} \approx 64\right)$ is approximately $12.5 \mathrm{~cm}$. This corresponds well to a fraction of human organ sizes. As a result, $B_{1}$ is affected by strong electromagnetic interactions like diffraction and reflection inside the body. These interactions lead to strong inhomogeneous excitation of the $B_{1}$ field due to interference patterns that arise and might cause destructive RF interference [27,28]. This inhomogeneity in excitation will disturb the image quality.

Another challenge at ultra high fields is the safety aspect. RF power deposition in human tissue increases as a function of magnetic field strength resulting in higher SAR levels which scales with the square of the field strength $\left(\mathrm{SAR} \propto B_{0}^{2}\right)$ [29].

To overcome the aforementioned challenges, several techniques have been proposed. The most widely considered are RF shimming [30,31], Transmit SENSE [32], and TIAMO [33]. These

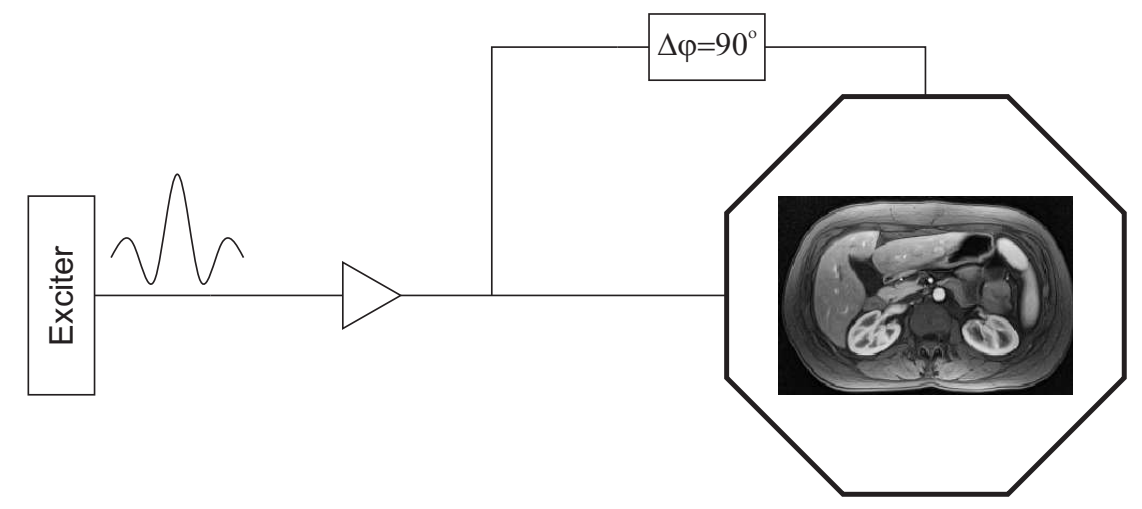

Figure 2.4: Quadrature excitation for RF body coil.

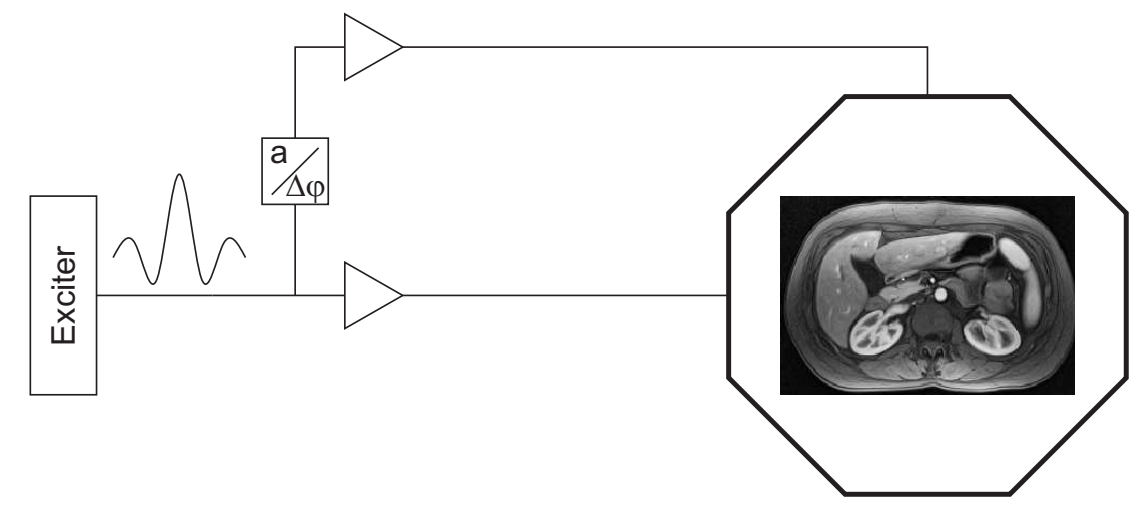

Figure 2.5: Two-channel RF shimming system. 
techniques drive the system by MultiTransmit parallel RF transmission technology, using arrays of multiple RF transmit/receive coils.

Before we discuss the parallel RF transmission technology, let us firstly discuss the conventional excitation for a classical MRI system. Fig. 2.4 shows the schematic for a conventional excitation system utilizing one RF exciter and one PA which drives the body coil at two different ports in quadrature manner. Quadrature drive ensures the circular polarized excitation to generate the circular polarized $B_{1}^{+}$field. A hybrid power splitter is responsible to split the power amplifier output signal into two identical signals with $90^{\circ}$ out of phase. This excitation technique works appropriately in case the coil is not loaded or loaded by a low a permittivity subject. As soon as we insert a high permittivity subject (i.e. human body), the homogeneity of RF excitation inside the subject gets distorted, and the purity of the circular polarized excitation will be lost. To recover the field homogeneity, we change the amplitude and the phase at one port of the coil as shown in Fig. 2.5, to get back the circular polarized excitation inside the subject. Usually, we avoid manipulating the RF signal at the high power side. Instead, we do it directly after the exciter at the small signal side before the PA.

For higher magnetic field strengths, additional degrees of freedom are required to achieve homogeneity. In general, we drive the RF coil by more transmit channels where the complex weighting (amplitude and phase) of each channel is adjusted aiming at more homogeneous $B_{1}$ field inside the subject. In this case, the resulting $B_{1}$ field inside the subject is a superposition of the $B_{1}$ fields generated by each channel element. Fig. 2.6 shows the example of an 8-element body coil array driven by 8 independent RF channels. The process of adjusting the phase and amplitude of the excitation signal in each transmit channel and applying these signals to the

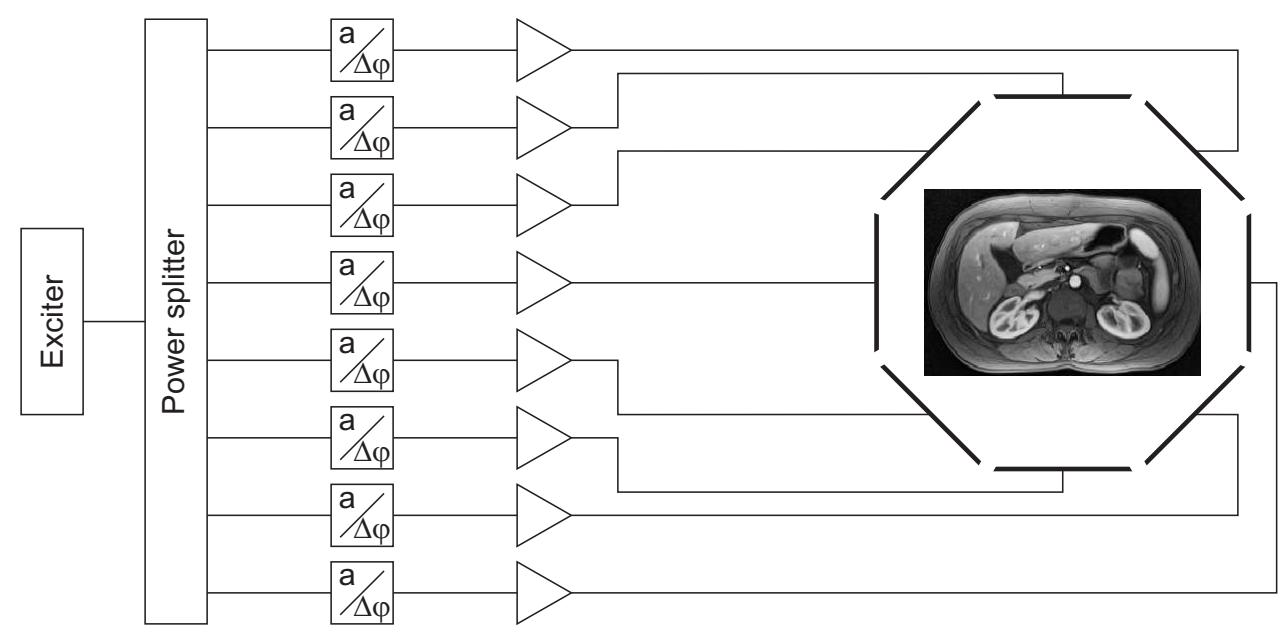

Figure 2.6: Static RF shimming. 


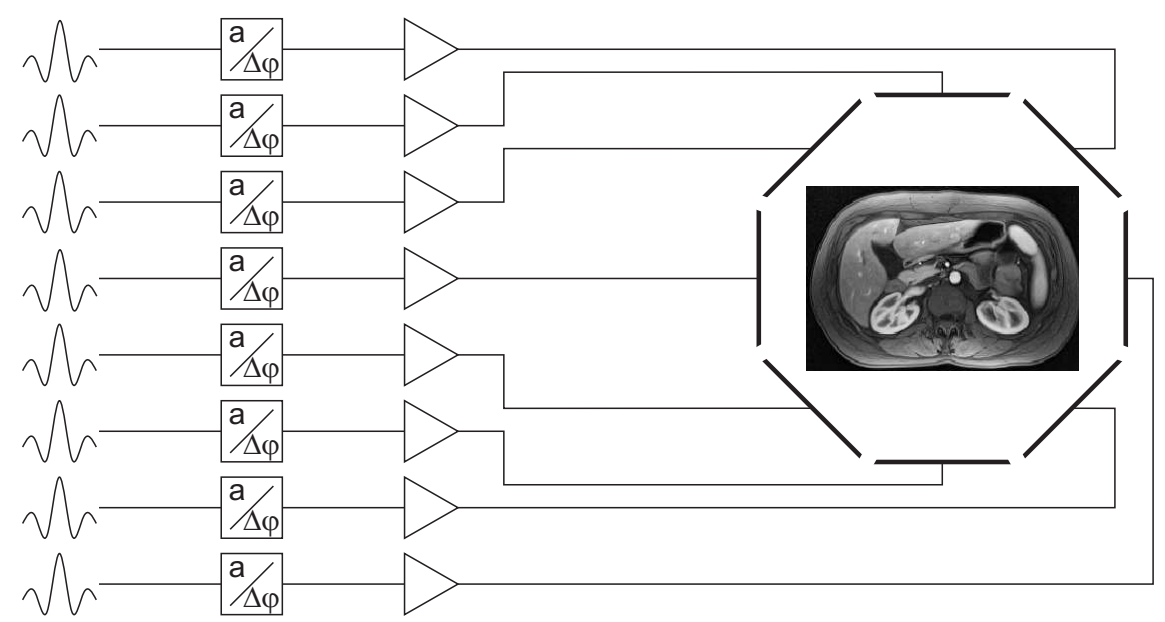

Figure 2.7: Dynamic RF shimming.

corresponding element in the RF coil array is known as RF shimming. Fig. 2.7 shows another technique proposed to reduce transmit $B_{1}$ inhomogeneity which is called dynamic RF shimming (Transmit SENSE). It is similar to RF shimming, but adds more degrees of freedom to the RF pulse envelope design. It applies arbitrary excitation profiles to each channel by manipulating the pulse envelope and leads to dynamic complex weighting of each channel as a function of time.

\subsection{High Q-factor MR Coils}

Recently, researchers in Ultra-high magnetic field MRI systems have preferred centrally-fed microstrip line elements [34,35], due to their high quality factor (Q) obtained from their narrowband design. This property increases the Signal-to-Noise Ratio (SNR) at receive which is essential to improve the quality of MR images. High transmit power efficiency can be obtained by well-tuning the RF coil to the operating frequency and well-matching the coil impedance to the $50 \Omega$ generator impedance.

In [36], a modification of the microstrip line element has been implemented by adding meanders at both ends of the microstrip lines. These meanders show their capability to decrease the coupling between neighbouring elements in a coil array in addition to some improvement in the penetration characteristic inside the sample.

In [37,38], a new version of centrally-fed meandered microstrip line elements has been proposed by loading the meanders with high-dielectric substrates. These substrates add advantage in terms of the safety aspect by reducing the specific absorption rate (SAR) values of the coil element.

More recently, Orzada has compared the field distribution of the aforementioned elements for a use in multichannel body coil array at 7 Tesla [39]. These three elements are shown in 


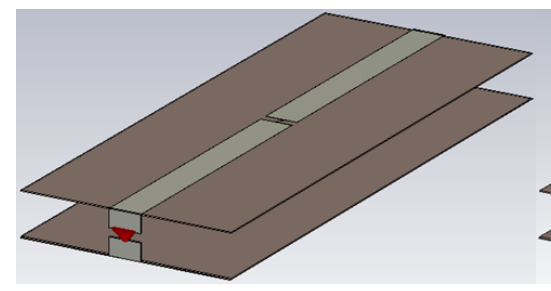

(a)

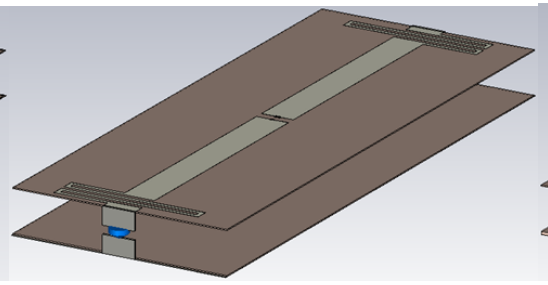

(b)

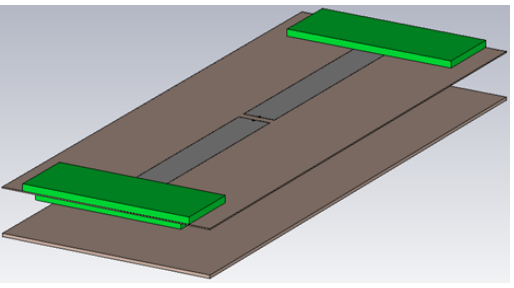

(c)

Figure 2.8: (a) MSL element, (b) Meander element, (c) Meander element with dielectric.

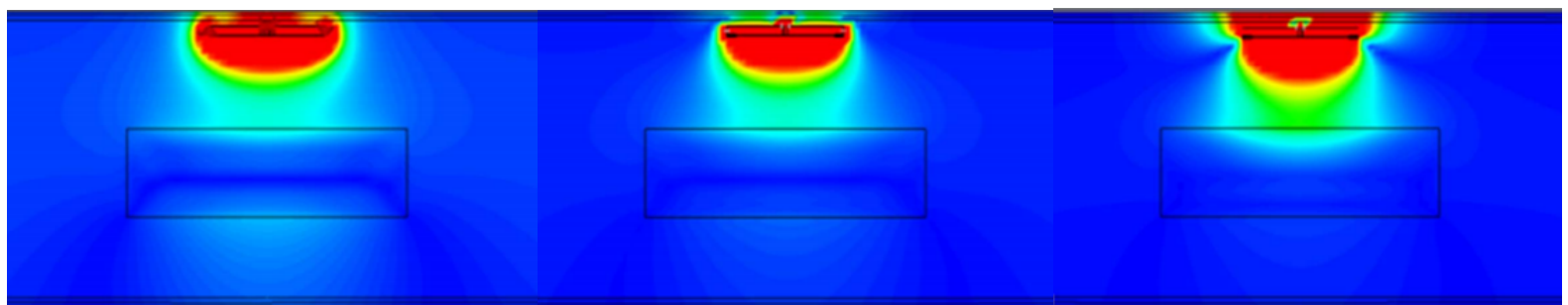

(a)

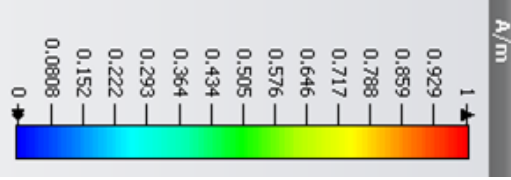

(b)

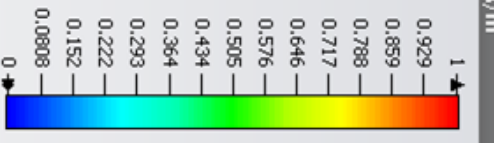

(c)

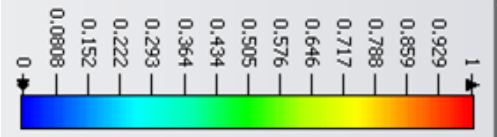

Figure 2.9: Simulated $|H|$ fields in a mid-sagittal section for (a) MSL element, (b) Meander element, (c) Meander element with dielectric. From [39]

Fig. 2.8. All the elements we fed with a $\lambda / 2$ balun and used a Brunner matching network [34]. Fig. 2.9 shows the simulated $|H|$ field distribution for the three different elements when each element is fed with $0.5 \mathrm{~W}$ power. It is noticeable that each element has a distinct field distribution. The first element shows a slightly broader field distribution leading to less focused $H$-field distribution towards the target region with higher radiation loss. The second element shows better focused $H$-field distribution with lower radiation loss. The third element shows a significant improvement in the field distribution where almost all the field is focused in the direction of the phantom with negligible radiation loss. The corresponding $\left|B_{1}^{+}\right|$distribution shows in Fig. 2.10. The best localized sensitivity can be obtained by the third element while a significant transmit sensitivity on the opposite side of the phantom appears by the first element.

Coupling between the transmit coil elements and mismatch of the loaded coils are considered a significant challenge for transmit coil arrays in a setup using a conventional transmit chain. In the following subsections we will discuss this issue by showing the effect on the new version of RF coil. 


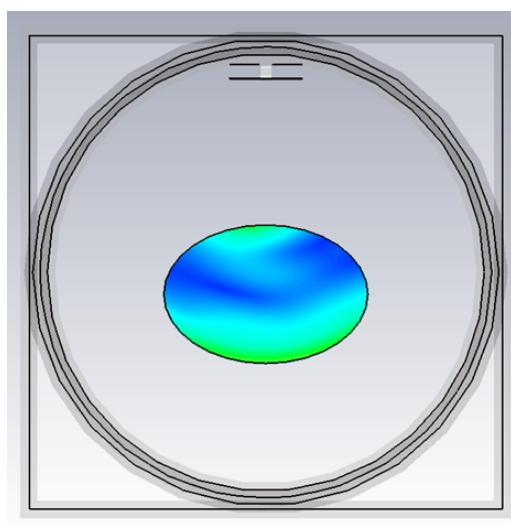

(a)

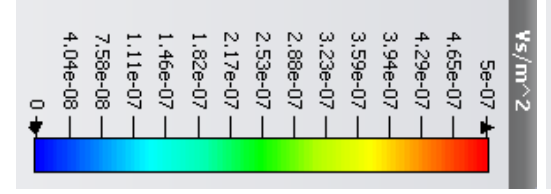

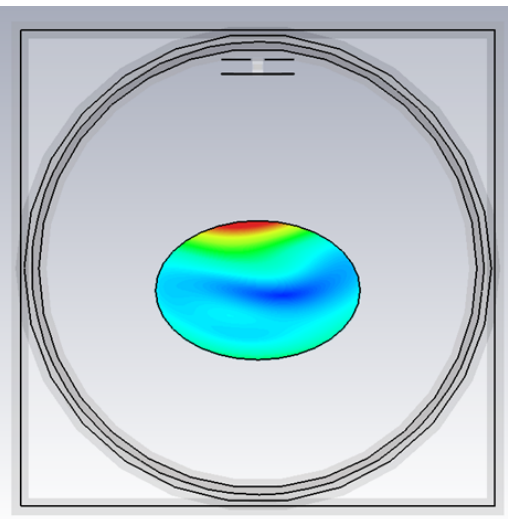

(b)

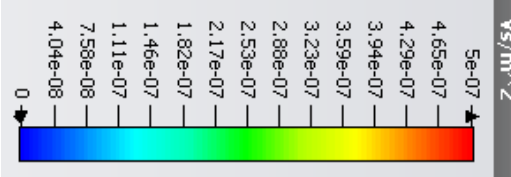

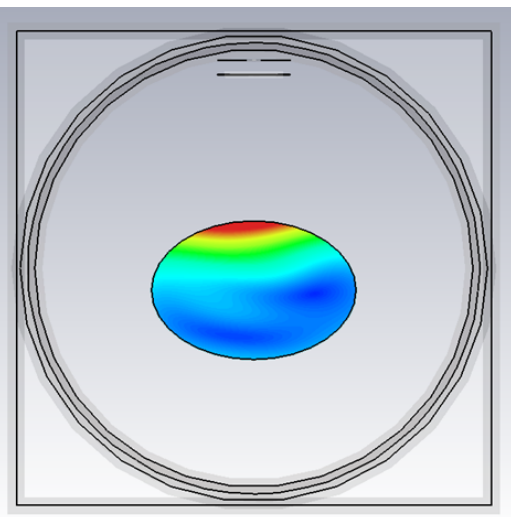

(c)

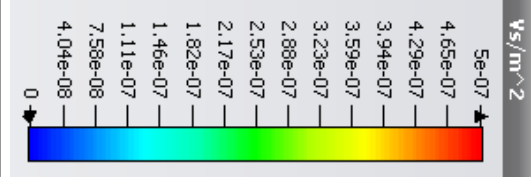

Figure 2.10: Simulated $\left|B_{1}^{+}\right|$fields in a mid-transversal section for (a) MSL element, (b) Meander element, (c) Meander element with dielectric. From [39]

\subsubsection{Loading Effect}

As is well-known from MR coils, phantom distance from the coil surface has a significant influence on the coil impedance and on the resonance frequency of the coil. Fig. 2.11 shows the simulation setup (using the FEM tool Ansoft HFSS) for a single meander coil [40], loaded by a homogeneous phantom $\left(\varepsilon_{\mathrm{r}}=45.3, \sigma=0.8 \mathrm{~S} / \mathrm{m}\right)$ with the dimensions of $(600 \mathrm{~mm} \times 90 \mathrm{~mm}$ $\times 370 \mathrm{~mm})$, placed at a distance $d$ above the coil and an aluminium plate $(600 \mathrm{~mm} \times 1 \mathrm{~mm} \times$ $370 \mathrm{~mm}$ ) is placed at a height $h$ below it. At $d=200 \mathrm{~mm}$ and $h=10 \mathrm{~mm}$, the coil has been tuned to the Larmor frequency for $7 \mathrm{~T}(298 \mathrm{MHz})$ and matched to $50 \Omega$. To demonstrate the loading effect, the distance $d$ has been decreased in steps. Two different cases are demonstrated. The first case is when the coil has been matched at the Larmor frequency at phantom distance $d=200 \mathrm{~mm}$. Then this distance has been decreased from $200 \mathrm{~mm}$ until $10 \mathrm{~mm}$ without any modification on the matching network. Fig. 2.12 (a) shows the reflection coefficient $\left(\mathrm{S}_{11}\right)$ as function of distance for this case, while Fig. 2.12 (c) shows the corresponding reflection coefficient $(\Gamma)$ on the Smith chart. The second case is when the coil has been matched at resonance, i.e., the matching network has been modified at each distance $d$. Fig. 2.12 (b) and (d) illustrate the reflection coefficient of this case. The second case shows more clearly the shifting of the resonance frequency of the coil when the phantom approaches the coil. An interpretation of the decrease of the resonant 


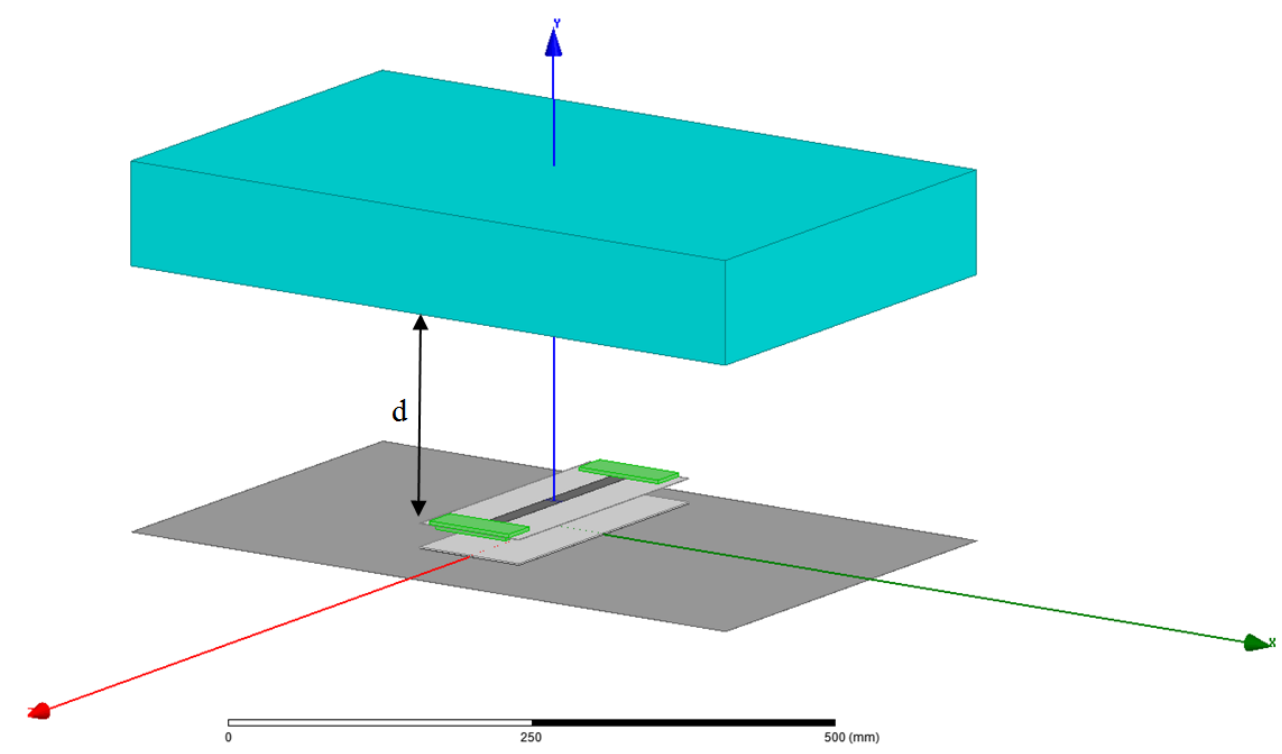

Figure 2.11: Simulation setup in HFSS software for single meander coil with a homogeneous phantom above the coil and RF shield below it.

frequency may recall the basic field distribution of the stripline coil which exhibits a strong electric stray field from one meander to the other meander. The stored electric energy of this field can be represented as a capacitance $C$ in an equivalent circuit which is a part of a series resonant circuit as shown in Fig. 2.13. With the field lines immersed in the high permittivity of the phantom, this capacitance increases leading to a decrease of the resonant frequency, where $f_{0}$ is given by:

$$
f_{0}=\frac{1}{2 \pi \sqrt{L C}}
$$

Because the phantom (or in general, the sample) medium is specified as lossy dielectric (complex permittivity) with conductivity, a dissipative (resistive) component appears which complements the coil resistance, leading to an increase of the coil series resistance $R_{\mathrm{c}}$ [40].

\subsubsection{Coupling Effect}

From the previous subsection, we have seen how the coil impedance is affected by loading. Another factor that also has a significant effect on the coil impedance is the mutual coupling between the coil array elements. Assume two parallel aligned coil elements with RF shield and phantom arranged in planar surfaces as shown in Fig. 2.14, where the gap width between the two 


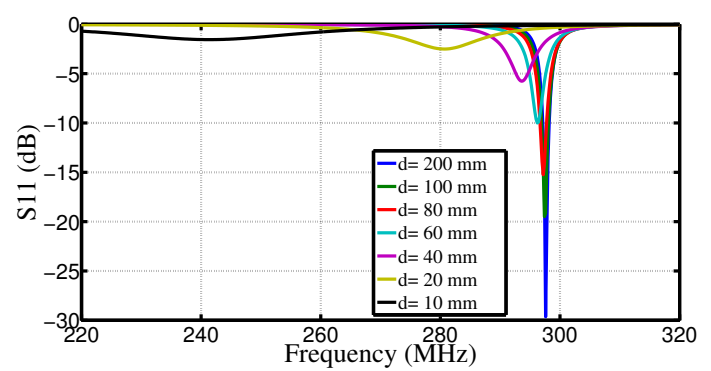

(a)

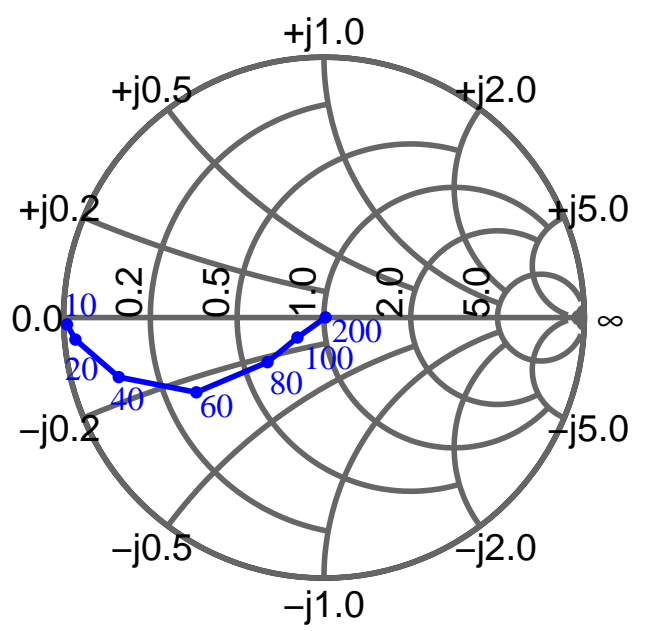

(c)

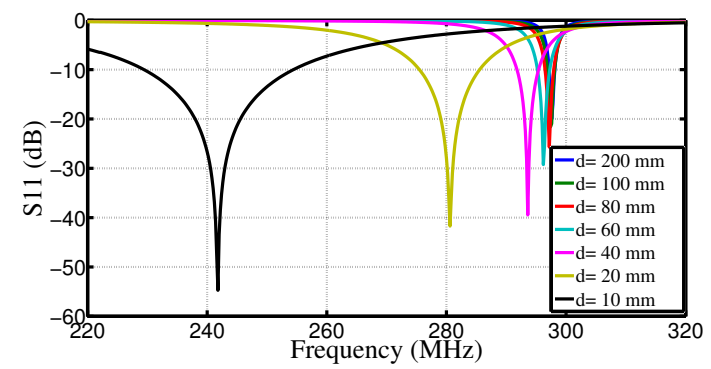

(b)

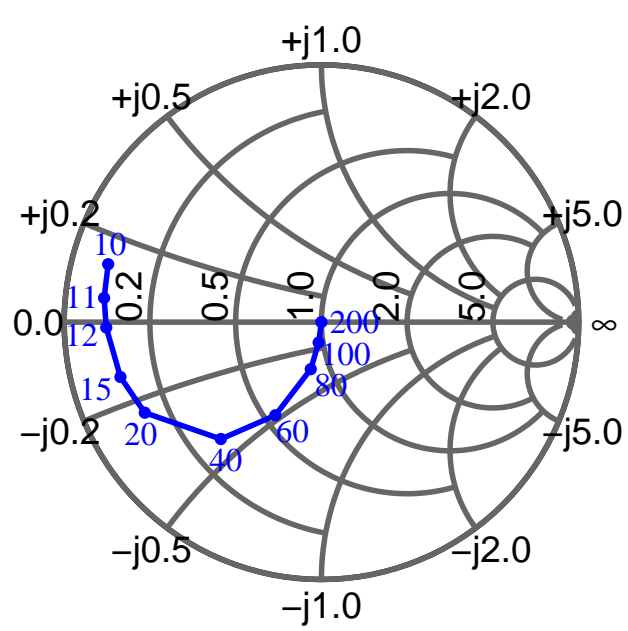

(d)

Figure 2.12: Demonstration of the loading effect. (a) Reflection coefficient $\left(S_{11}\right)$ vs. frequency as function of distance while the coil is matched at the Larmor frequency, (b) reflection coefficient $\left(S_{11}\right)$ vs. frequency as function of distance while the coil is matched at resonant frequency, (c) the corresponding reflection coefficient $(\Gamma)$ for the first case on the Smith chart, $(d)$ the corresponding reflection coefficient $(\Gamma)$ for the second case on the Smith chart.

elements $g=100 \mathrm{~mm}$, the distance between the phantom and the coil surface $d=200 \mathrm{~mm}$, and the height of the two elements above the RF shield $h=10 \mathrm{~mm}$, measured from the coil ground plane. From antenna theory, two common equivalent networks can model the coupling between elements: $T$-network and pi-network [41]. Fig. 2.15 (a) shows the $T$-equivalent network model for two coupled active elements where the relationship between the voltages and currents across the element ports can be shown in the following based on the impedance matrix:

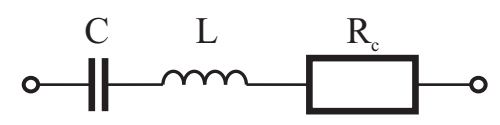

Figure 2.13: Series resonant circuit behaviour of meander coil. 


$$
\left[\begin{array}{l}
V_{1} \\
V_{2}
\end{array}\right]=\left[\begin{array}{ll}
Z_{11} & Z_{12} \\
Z_{21} & Z_{22}
\end{array}\right]\left[\begin{array}{l}
I_{1} \\
I_{2}
\end{array}\right]
$$

where $V_{\mathrm{i}}$ and $I_{\mathrm{i}}$ are the voltage and current across the $\mathrm{i}^{\text {th }}$ element port, $Z_{\mathrm{ii}}$ is the self-impedance of the $\mathrm{i}^{\text {th }}$ element (typically a tuned and matched coil), $Z_{\mathrm{i}}$ is the input impedance seen at $I_{\mathrm{i}}$ port and due to reciprocity, $Z_{\mathrm{ij}}=Z_{\mathrm{ji}}$, where $\mathrm{i} \neq \mathrm{j},\{\mathrm{i}, \mathrm{j}\}=1,2$. To demonstrate the coupling effect on

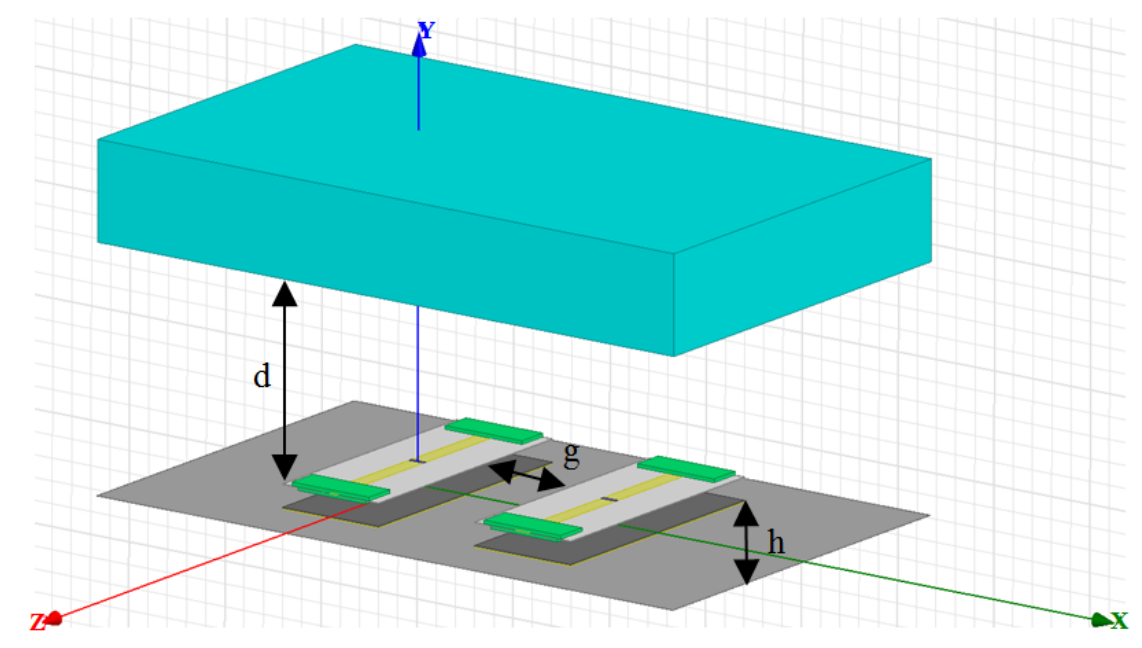

Figure 2.14: Simulation setup in HFSS software for two-coupled meander coils

the coil impedance, let us calculate the (coupled) current $I_{\mathrm{m}}$ passing through the matched coil element as a result of coupling from the active coil element as seen in Fig. 2.15 (b). Based on Kirchhoff's law, the voltage $V_{1}$ can be described by

$$
V_{1}=I_{1}\left(Z_{11}-Z_{21}\right)+\left(I_{1}+I_{\mathrm{m}}\right) Z_{12}
$$

and the coupled voltage $V_{\mathrm{m}}$ produced across the matched load impedance $Z_{\mathrm{L}}$ can be described by

$$
V_{\mathrm{m}}=Z_{21} I_{1}+Z_{22} I_{\mathrm{m}}=-I_{\mathrm{m}} Z_{\mathrm{L}}
$$

By rearranging Eq. 2.4, the coupled current $I_{\mathrm{m}}$ produced in the matched terminated element is 


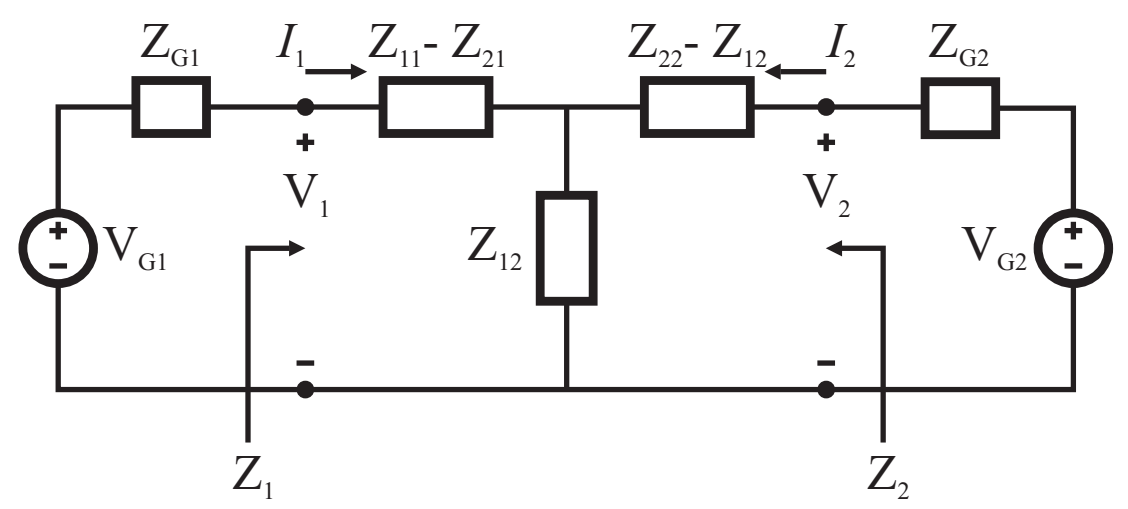

(a)

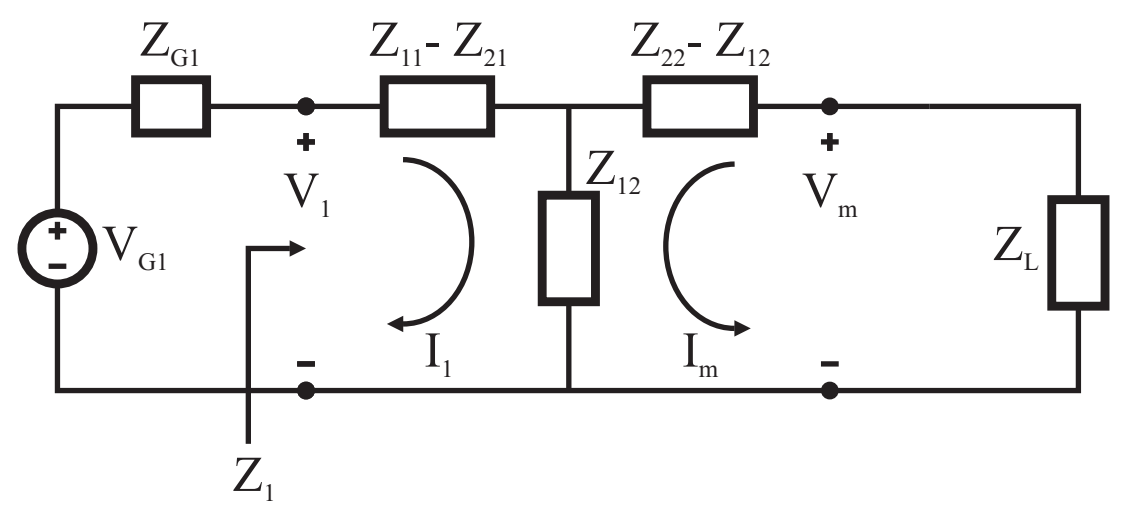

(b)

Figure 2.15: $T$-equivalent network model for two coupled coil array elements. (a) Two active elements array, (b) one active element (left) and one matched terminated element (right).

$$
I_{\mathrm{m}}=\frac{-Z_{12}}{Z_{\mathrm{L}}+Z_{22}} I_{1}
$$

By substituting Eq. 2.5 into Eq. 2.3 and rearranging, the input impedance $Z_{1}$ can be expressed as

$$
\frac{V_{1}}{I_{1}}=Z_{11}-\frac{Z_{12}^{2}}{Z_{L}+Z_{22}}
$$

Returning back to Fig. 2.15 (a), assume the second coil element is matched terminated by $Z_{\mathrm{L}}$, then the input impedance $Z_{1}$ can be calculated as a ratio of the voltage $V_{1}$ across the $1^{\text {st }}$ element port to the coil current $I_{1}$ (Eq. 2.6) which is made equal to $50 \Omega$ by matching both coils under coupling. This case is identical to a case when the second coil element is connected to generator with input power $P_{\mathrm{G} 2}=0 \mathrm{~W}$. If this generator starts to feed the coil element with a certain power, 
the coupled voltage $V_{\mathrm{m}}$ and current $I_{\mathrm{m}}$ will superimpose the voltage $V_{1}$ and current $I_{1}$ of the first case leading to a change in the ratio of the voltage across port 1 and the current in coil 1 . Thus, the input impedance $Z_{1}$ will change accordingly. A demonstration of coil impedance $Z_{1}$ variation or input reflection coefficient $\Gamma_{1}$ variation due to coupling effect is shown in Fig. 2.16. This demonstration has been done for the coil configuration in Fig. 2.14, where the coupling coefficient is approximately $-18 \mathrm{~dB}$. The first coil element has been driven by $10 \mathrm{dBm}$ input power while the second coil element has been driven in steps of power and by varying the phase through one full cycle.

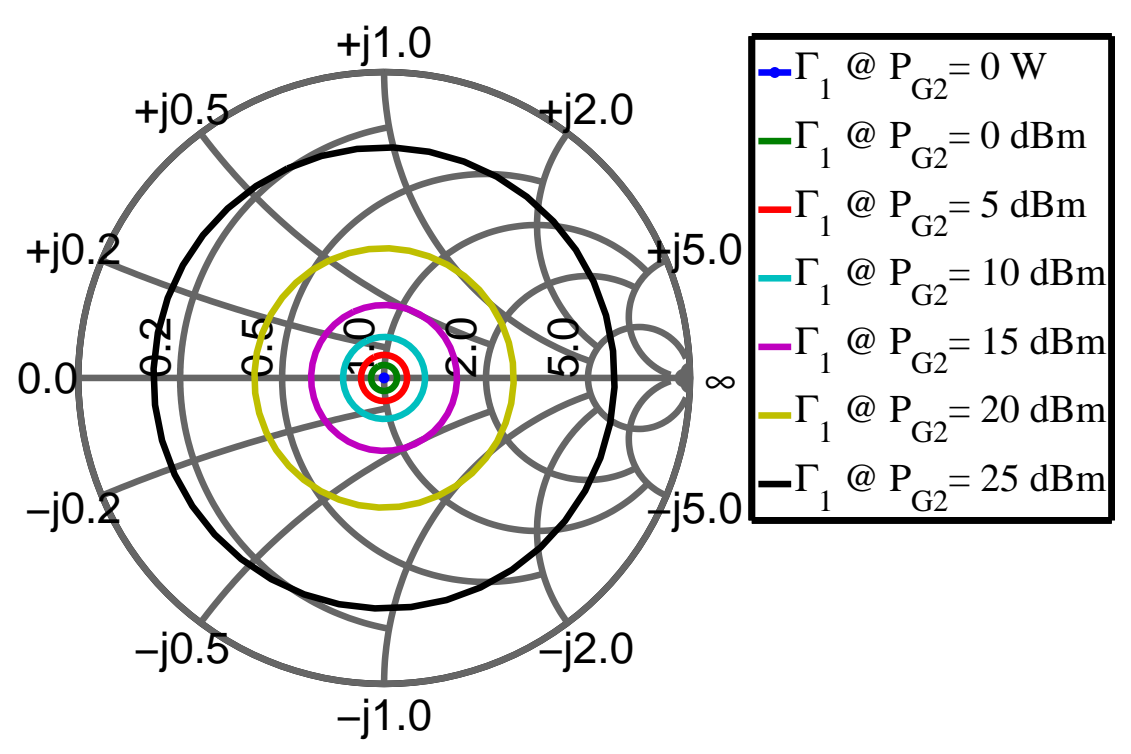

Figure 2.16: Reflection coefficient of a matched coil due to mutual coupling as function of power into the second coil.

\subsubsection{Equivalent Circuit Model of Coupled Meander Coils}

To verify whether the equivalent network model that has been described in the previous subsection is applicable to our coupled coil array elements, we have developed an equivalent circuit employing series RLC resonators and a $T$-network. A series RLC resonator is used to model each coil element, whereas the $T$-network is used to model the interaction between the two coil elements as seen in Fig. 2.17. The meander coil parameters such as reflection coefficient, input impedance, Q-factor, etc. have been identified by a simulation using HFSS for single coil. The resonant frequency of $298 \mathrm{MHz}$ and the Q-factor of 180 obtained by the EM simulation have been used in the equivalent circuit as initial values. Optimization goals for the equivalent circuit model include conditions on the resonant frequencies and Q-factors of the coils in addition to 


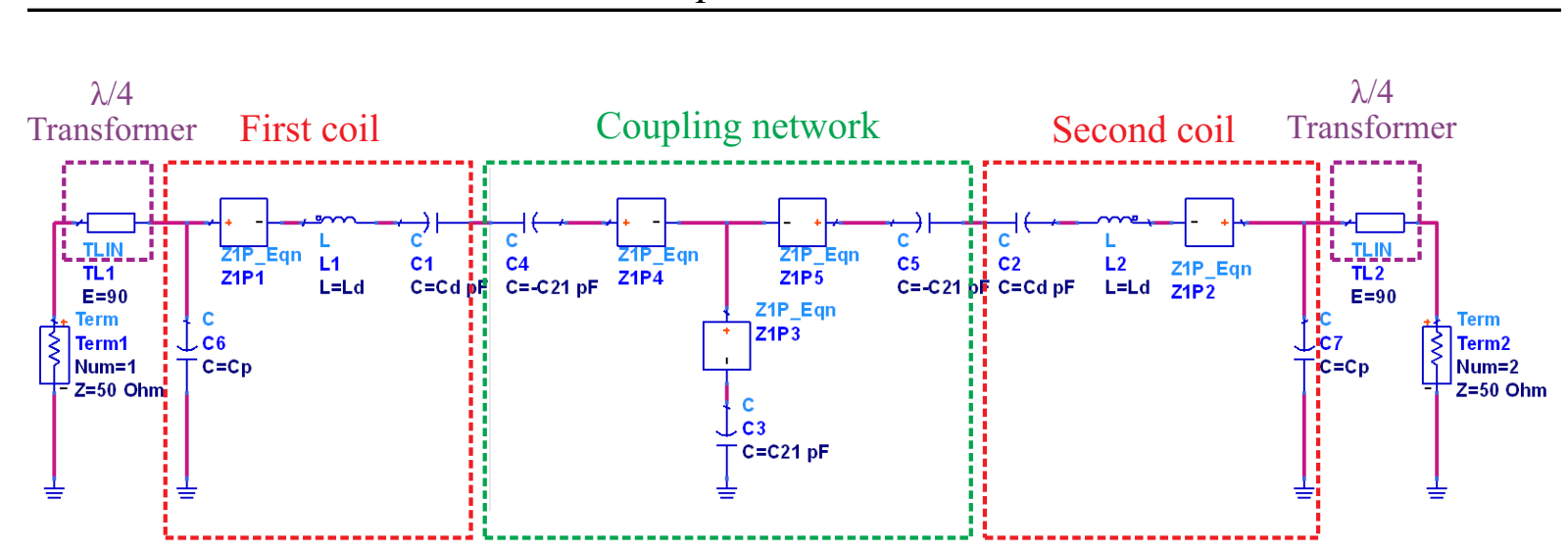

Figure 2.17: Equivalent circuit model of two-parallel meander coils.

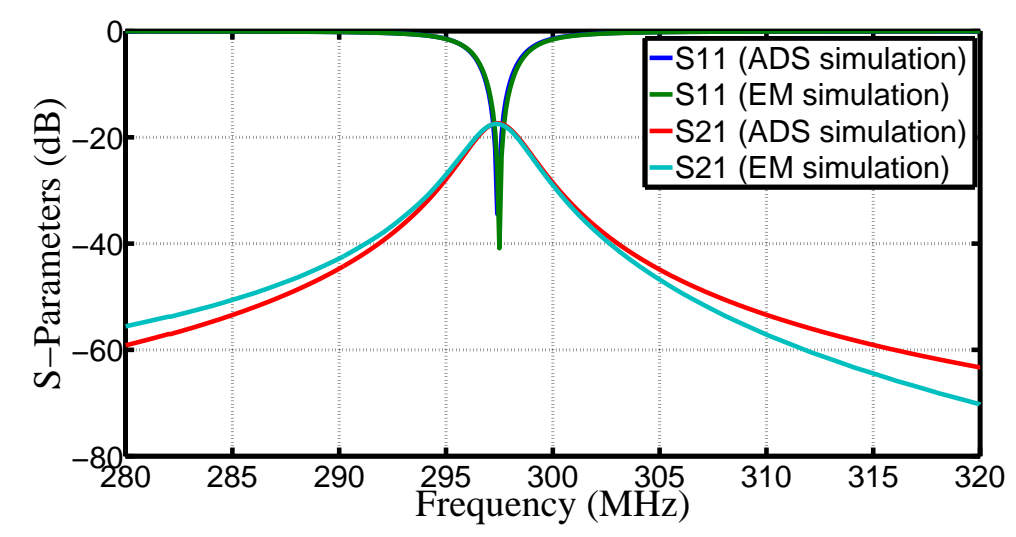

Figure 2.18: $S_{11}$ and $S_{21}$ based on ADS simulation and EM simulation.

a good agreement between EM-simulation and circuit simulation (ADS simulator) relating to the mutual impedance between the two elements. Fig. 2.17 shows the equivalent circuit model obtained using ADS simulator for the coils configuration in Fig. 2.14. A series RLC resonator consists of $Z 1 P 1=Z 1 P 2=R_{\mathrm{d}} \cdot\left(f / f_{0}\right)^{2}$ where $R_{\mathrm{d}}=2.5 \Omega, L 1=L 2=256.3 \mathrm{nH}, C 1=C 2=1.12 \mathrm{pF}$, and an additional shunt capacitor to represent the stray electric fields between the two halves of the meanders $C 6=C 7=3.79 \mathrm{pF}$ [42]. The $T$-network consists of $C 3=-C 4=-C 5=863.6 \mathrm{pF}$, $Z 1 P 3=-Z 1 P 4=-Z 1 P 5=R_{\mathrm{m}} \cdot\left(f / f_{0}\right)^{2}$ where $R_{\mathrm{m}}=0.194 \Omega$. A quarter-wave transformer is utilized as the matching network with characteristic impedance $T L 1=T L 2=11.7 \Omega$. Fig. 2.18 shows how the equivalent network behaves well with the EM model.

From this section (2.4), we can conclude that the coil impedance varies considerably due to loading and coupling in an array. This is a problem because the delivered PA power decreases due to return loss, the phase of generated $B_{1}$ varies due to change in resonant frequency or due to out-of-phase currents induced by neighbor coils. As a consequence, the design of the PA should allow compensation of such degradation effects (discussed in Ch. 3-5). 


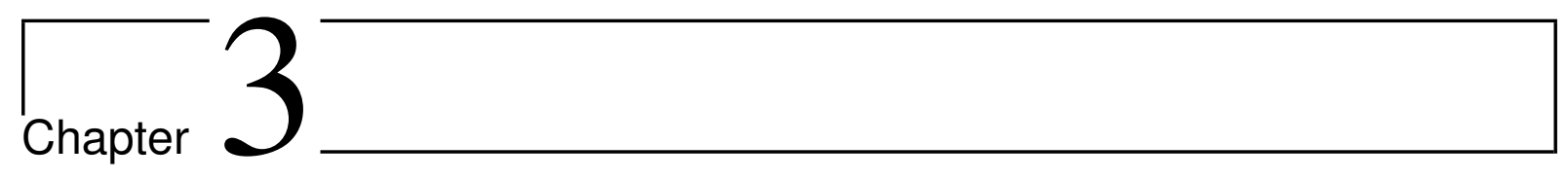

\section{Conventional RF Power Amplifiers}

\section{FOR MRI}

\subsection{Introduction}

In the RF transmitter system, the radio frequency power amplifier (RFPA) is considered as the heart of the MRI scanner. It is responsible to amplify the frequency synthesizer signal to sufficient power in order to excite the nuclei of the tissue. The amount of power required from the power amplifier depends on the magnetic field strength and the body volume to be imaged. In case of ideal power amplifiers, the output signal would be a perfect replica of the input signal scaled up by a factor. In contrast, conventional RFPAs behave non-linearly, causing distortion of the waveform being transmitted.

For MRI, RFPAs are optimized for pulse operation to provide high RF power pulses within a short period of time. The maximum duty cycle of the RF pulses are on the order of 10$15 \%$. Pulse RF amplifiers have different design criteria compared to continuous wave (CW) RF amplifiers in terms of power supply and heat management. The demand for high average power from CW amplifiers requires large DC power supplies in addition to large heat sinks with fan or liquid heat exchanger to remove heat from the transistor. In terms of RF circuitry, CW amplifiers and pulse RF amplifiers share the same circuit design. 


\subsection{The Architecture of RFPAs for MRI}

Since the RFPAs in MRI operate in pulse mode, the RF source at the input side would synthesize a pulsed, amplitude modulated RF signal as seen in Fig. 3.1. After the signal is synthesized with appropriate amplitude generally on the order of $0 \mathrm{dBm}$, frequency and phase, it has to be amplified by an RFPA to a sufficient power level in the $\mathrm{kW}$-range in order to excite a target tissue. High power pulses with short pulse duration are required to achieve the desired flip angles. Once the pulsed RF signal reaches the RFPA, it passes through two low power amplifier stages (pre-driver and driver) to amplify the input signal from low level to higher level which is enough to drive the high power amplifier output stage. The microcontroller (MC) is responsible for monitoring important RFPA operating parameters such as: DC bias, transistor temperature, RF output power, etc. In case of fault operation mode, it switches off the PA as a safety procedure. A directional coupler is used to monitor the forward and reflected power to guarantee safe operation. The DC power supply provides all the amplification stages with appropriate DC power. [43]

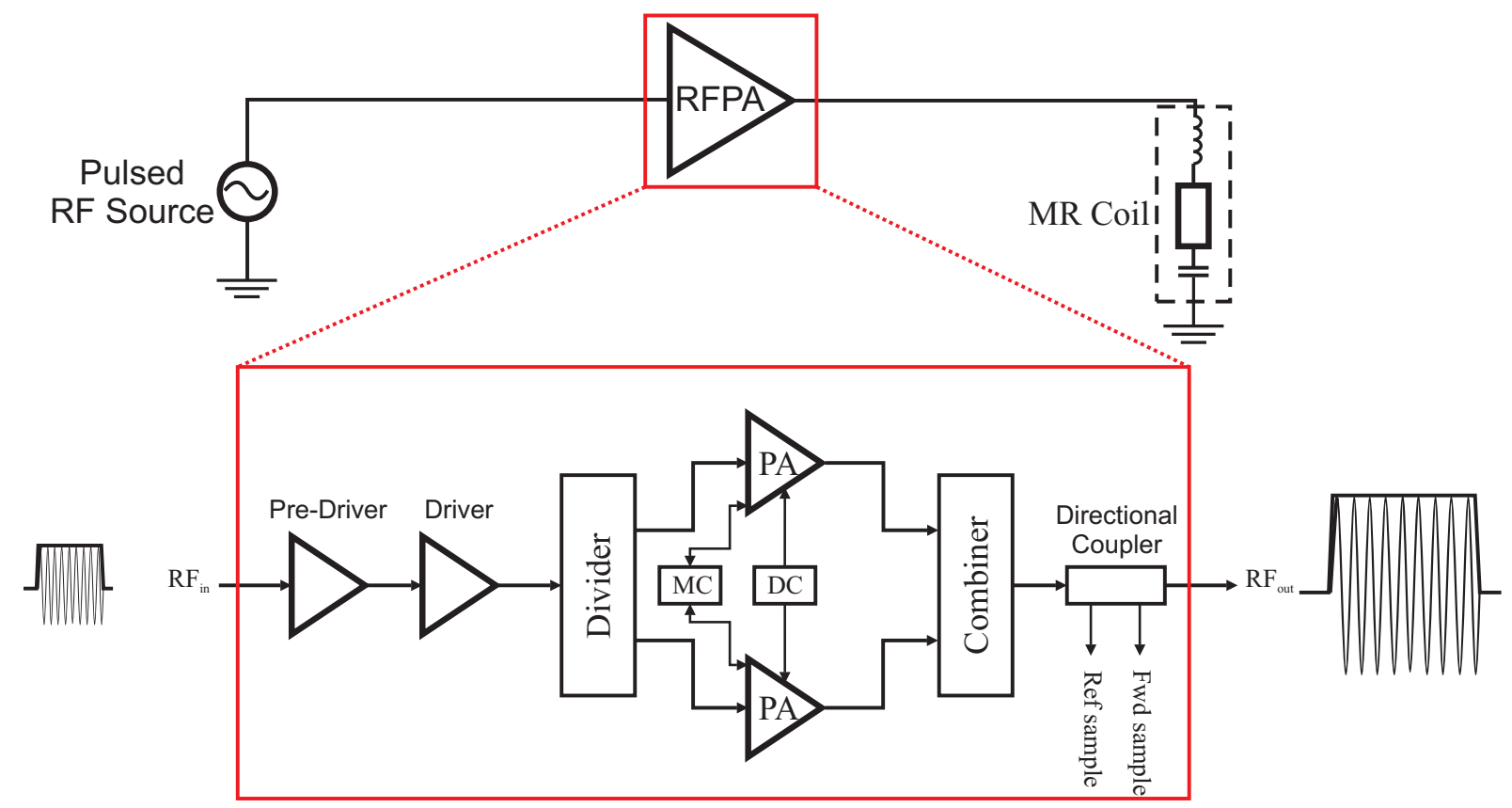

Figure 3.1: Block diagram of RFPA architecture.

\subsection{RFPA Classes}

RF power amplifiers are classified based on the bias condition of their last stage transistors, which in turn determines their operating point. By choosing the operating point, the performance 
of the power amplifier can be evaluated in terms of linearity and efficiency. The term linearity refers to the purity of amplification (i.e. distortion level), whereas the term efficiency refers to the ability to convert the DC power into RF signal power. The operating point is related directly with the specification of the conduction angle of the drain current for various amplifier classes. Designing power amplifiers requires a trade-off between efficiency and linearity depending on the application. For wireless mobile communication based on non-constant envelope modulation, linear PAs are required, whereas highly efficient amplifiers are used in phase or frequency modulation systems which require high efficiency, e.g., in satellite application [44]. For MRI applications, where RFPAs operate in pulse mode, moderately high linear amplification is demanded to meet the pulse sequences specifications in terms of amplitude and phase stability throughout each pulse and the pulse sequence duration.

\section{Class A Operation}

The transistor operating class A is characterized by high linearity because the operating point at $I_{\max } / 2$ is set half way of the linear region of its transfer function characteristic as seen in Fig. 3.2. Such operating point provides a distinct conduction angle $\theta_{c}=2 \pi$. From the power efficiency point of view, class A amplifiers have low efficiency $(<35 \%)$ due to their high power dissipation, which requires due consideration of heat handling.

\section{Class B Operation}

The transistor operating class B has no DC bias current. Its operating point is set close to the threshold voltage as seen in Fig. 3.2. This means, the transistor conducts for one half of the input waveform with conduction angle equal $180^{\circ}$. In this case, the linearity decreases whereas the power efficiency increases (ca. 70\%).

\section{Class AB Operation}

The class AB amplifier is considered a compromise between class A and class B amplifiers. It offers a flexible solution for a trade-off between acceptable linearity and efficiency. The operating point for this class is set between class A and class B operating points. Therefore, the conduction angle extends between $180^{\circ}$ and $360^{\circ}$. The obtainable efficiency from this class varies between $50 \%$ and $70 \%$.

Extremely non-linear amplifiers (class D,E) that operate in switch mode are characterized by 
high power efficiency (> 90\%). In order to make them usable for MRI, a complex linearisation technique is required.

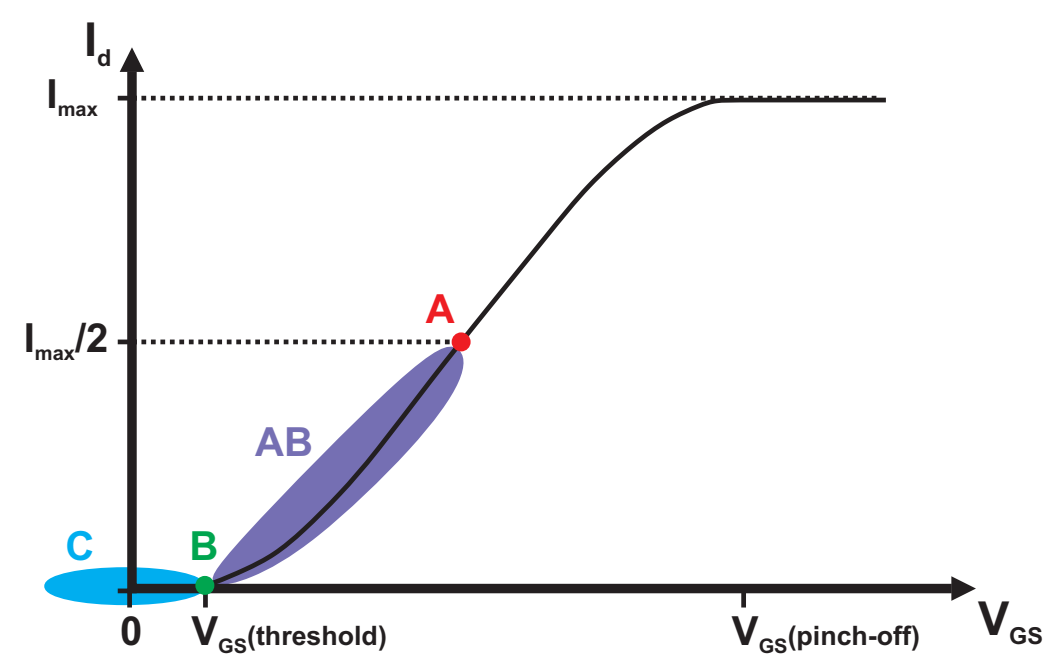

Figure 3.2: Operating points of an enhancement mode FET for different classes.

\subsection{Push-Pull Amplifier}

Since Ultra-high magnetic field MRI scanners demand power amplifiers that have a capability to offer at least $1 \mathrm{~kW}$ on each transmit channel, power transistors in push-pull configuration are highly recommended. A push-pull amplifier is an amplifier which employs two identical transistors where the transistors conduct alternately for their respective half cycle of the RF input signal. This feature improves the linearity of the power amplifier (e.g., class $A B$ and B) because the transistors conduct alternately for the entire input cycle to reproduce the entire waveform at the output. The output power capability of these amplifiers is twice that obtained by the single-ended amplifiers. Thus, these power amplifiers are considered as good candidates to be used in situations where high output power, with high linearity and efficiency are required. Push-pull amplifiers can be biased to operate in the different classes of operation (A, AB, B, or C). The most common class in MRI is class AB, where a good linearity is obtainable without huge penalties in dissipation heating [45-47].

\section{Theory of operation}

A power amplifier based on a push-pull design consists of two identical transistors (e.g., an LDMOSFET) as seen in Fig. 3.3. In case the transistors are biased to zero DC current, this ensures that both transistors are in cut-off region and operate in class B mode. Once a full cycle 
RF signal is applied to the input as shown in Fig. 3.3, it is split into two antiphase signals by passing through the Balun which works as power splitter and phase shifter. A Balun (Balanced Unbalanced) is an electrical device that is used to couple a balanced system that is symmetrical with respect to ground, and unbalanced system with one side grounded. The balanced signal passes through the matching network before driving the transistors. The input matching network is designed based on the complex-conjugate matching technique to provide maximum gain, whereas the output matching network is designed based on the large-signal power matching technique to achieve maximum power transfer. The idea behind using such a matching technique at the output side is that the optimum load impedance varies as a function of output power. Once the balanced signal reaches the gate terminal of both transistors, the upper transistor will conduct during the positive half cycle and the lower transistor will conduct during the negative half cycle due to the signal inversion. Since each transistor amplifies half cycle of the input signal, the output balun combines the two halves to produce the entire waveform. From Fig. 3.3, we notice that the output signal is distorted in the region where the current conduction crosses from one transistor to another. This occurs because the DC bias current is zero, and both transistors gradually move into conduction when the input signal increases above their threshold voltage. Such distortion is known as crossover distortion and appears as a clipping effect on the output waveform within a period of time between the positive and negative alteration of the input signal.

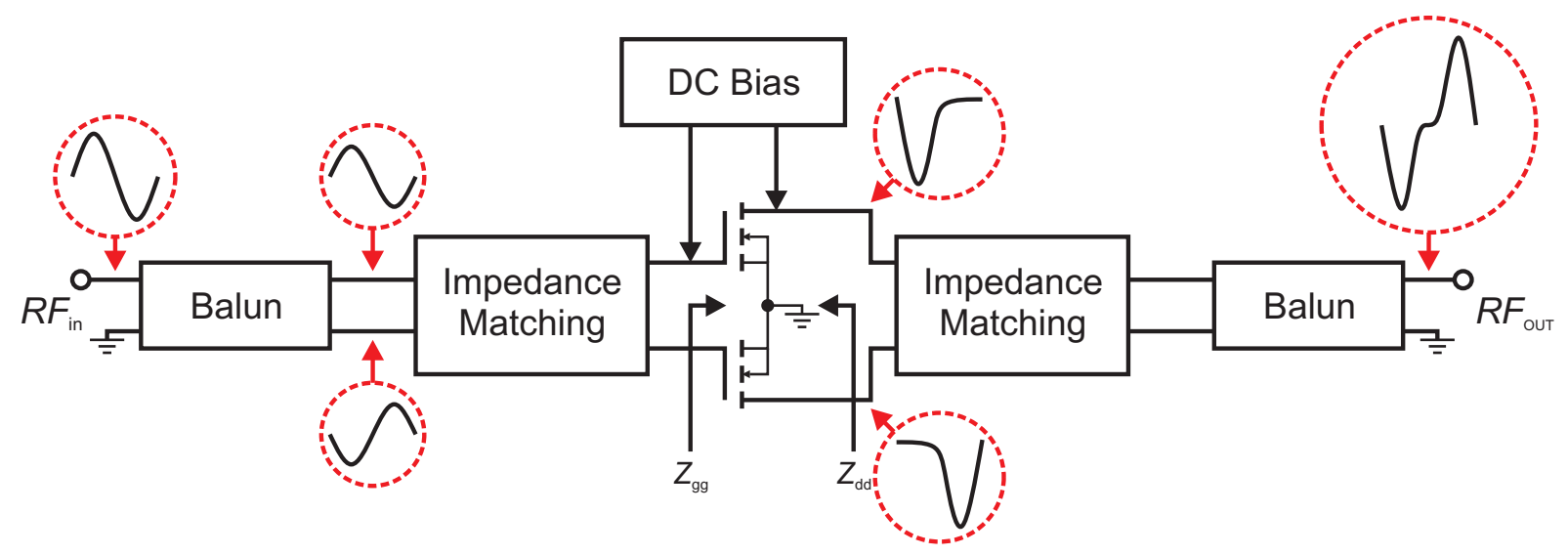

Figure 3.3: Block diagram of Push-Pull amplifier.

To overcome crossover distortion present in class "B" amplifier design, both transistors should be biased slightly above threshold to operate in class AB mode. This ensures slight conduction of both transistors even when no input signal is present. Fig. 3.4 and Fig. 3.5 show the output characteristics for push-pull amplifiers operating in class B and class AB modes respectively. Fig. 3.4 shows how the crossover distortion is produced on the output waveform 
when the operating point is set close to the threshold voltage in order to operate the transistors in class B mode, whereas Fig. 3.5 shows how the crossover distortion is eliminated when the operating point is placed slightly higher than threshold.

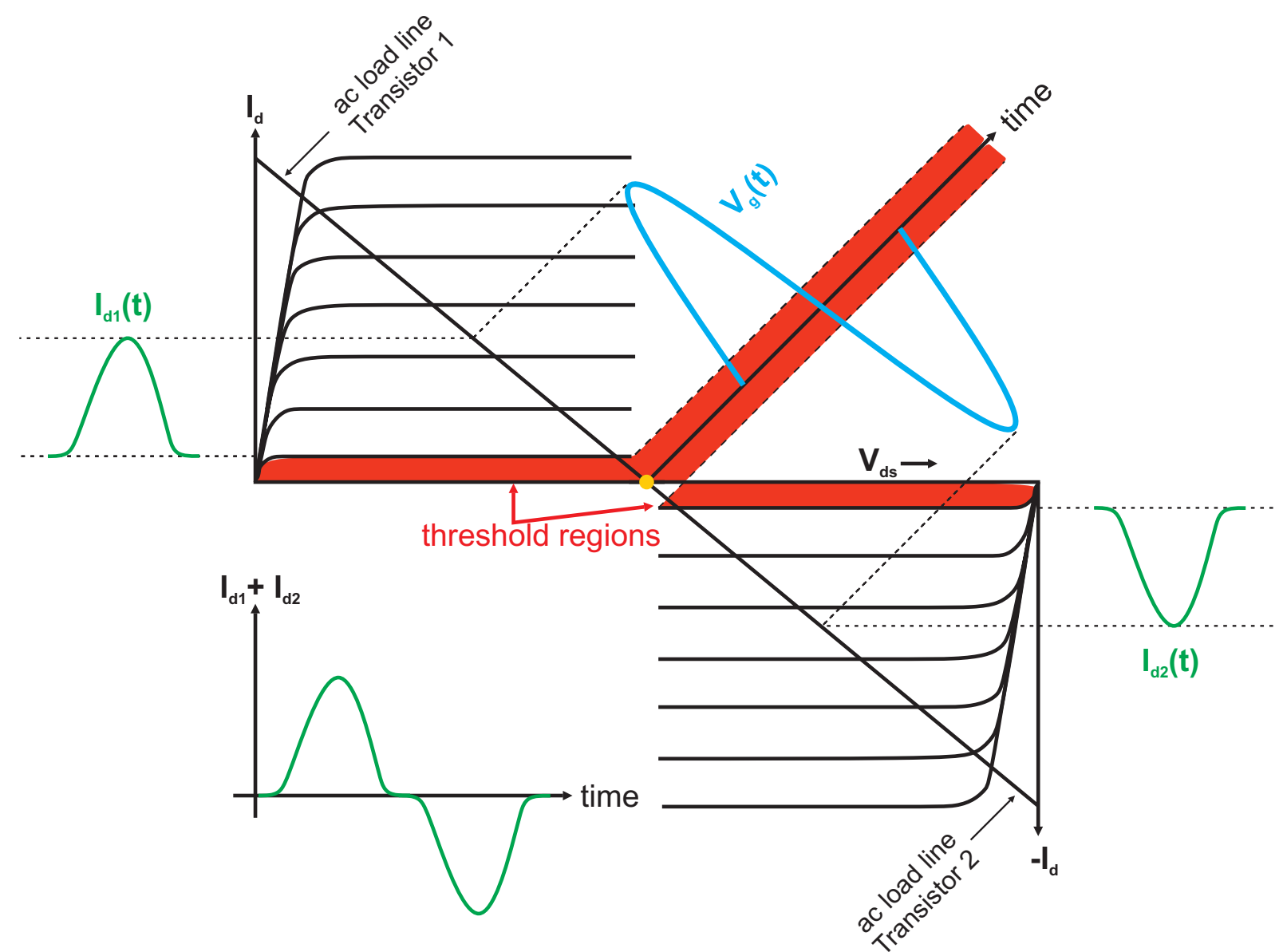

Figure 3.4: Output characteristic with output wave forms for push-pull amplifier operating in class B mode.

\section{Advantages of push-pull amplifiers}

- Input and output impedances of transistors in push-pull configuration $\left(Z_{\mathrm{gg}}\right.$ and $\left.Z_{\mathrm{dd}}\right)$ are four times higher than that for the single ended transistor [48]. This can be easily proven by placing a balanced center-tapped transformer between the input and the gate terminals of both transistor as shown in Fig. 3.6 (a). A center tapped transformer has a center tapped secondary, producing two separate output voltages which are identical in magnitude, but with anti-phase polarity. For any ideal transformer as in Fig. 3.6 (b), the following equation is valid 


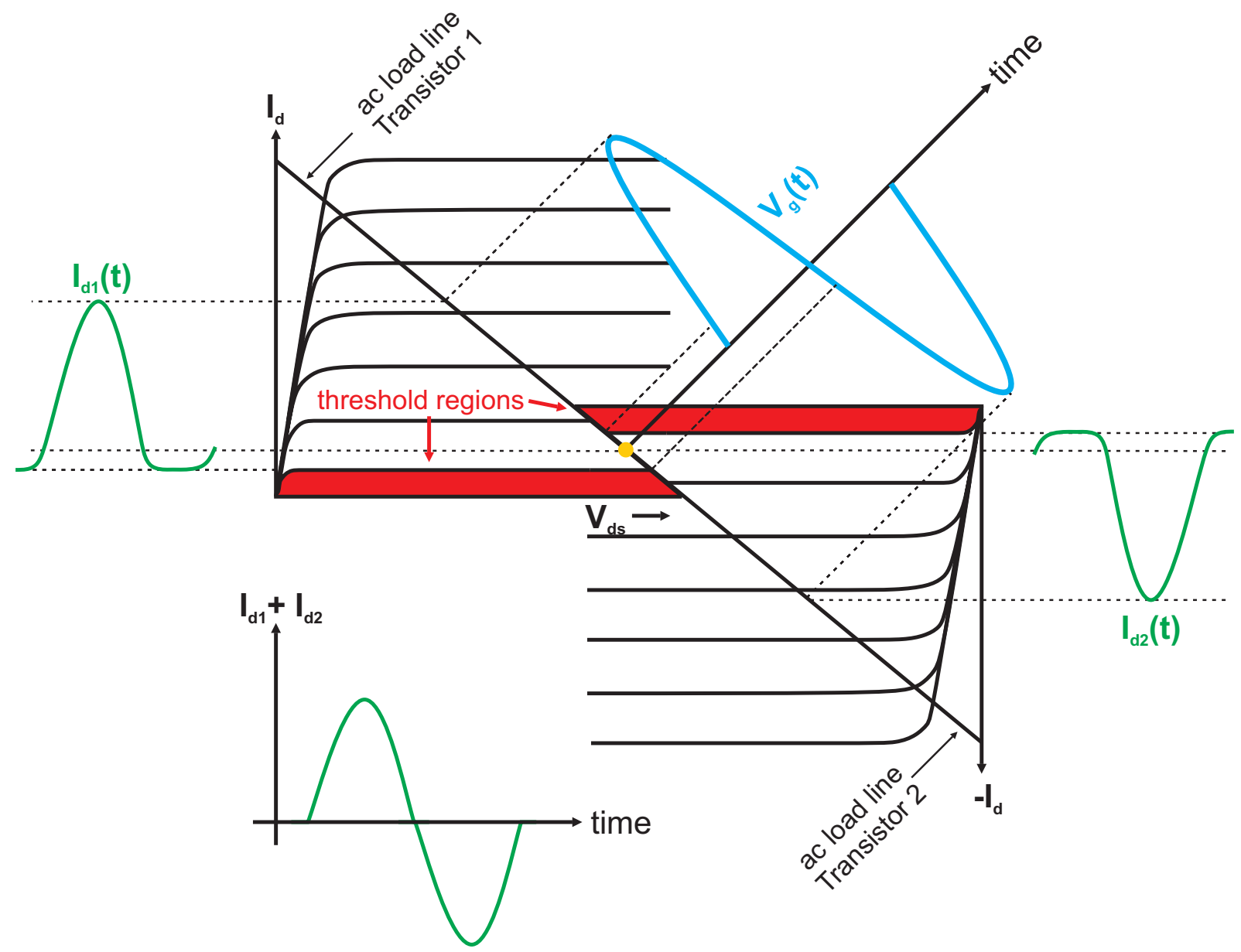

Figure 3.5: Output characteristic with output wave forms for push-pull amplifier operating in class AB mode.

$$
\frac{V_{\mathrm{p}}}{V_{\mathrm{s}}}=\frac{N_{\mathrm{p}}}{N_{\mathrm{s}}}=\sqrt{\frac{Z_{\mathrm{p}}}{Z_{\mathrm{s}}}}
$$

where $N_{\mathrm{p}} / N_{\mathrm{s}}$ is the turns ratio between the primary and the secondary sides, $V_{\mathrm{p}} / V_{\mathrm{s}}$ is the voltages ratio, and $Z_{\mathrm{p}} / Z_{\mathrm{s}}$ is the impedances ratio. For a case of center tapped-transformer with the turns ratio of 1 , the voltage ratio between the primary and a half of the secondary is $V_{1} / V_{2}=2$, whereas the impedances ratio is

$$
Z_{1}=Z_{2}\left(\frac{n_{1}}{n_{2}}\right)^{2}=4 Z_{2}
$$

With this result, we conclude that the impedance matching for push-pull amplifier is easier 
than that for a single ended transistor. In addition, the virtual ground in push-pull amplifiers makes the matching network topology more simpler and compact.

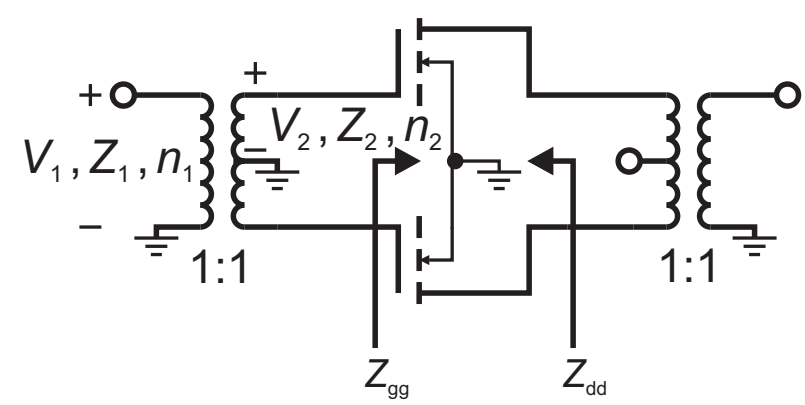

(a)

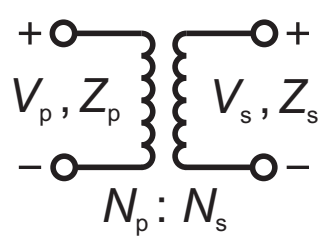

(b)

Figure 3.6: (a) Push-pull amplifier using balanced center-tapped transformers, (b) ideal transformer.

- The push-pull amplifier with symmetrical circuit configuration eliminates the DC component and all even order harmonics (2nd, 4th, etc.) in the output [49].

To prove the correctness of this feature, both transistors are assumed to be identical. The Fourier series expansion for the drain current of the upper MOSFET transistor can be written as

$$
\begin{aligned}
i_{\mathrm{D} 1} & =I_{\mathrm{D}}+i_{\mathrm{d} 1}+i_{\mathrm{d} 2}+i_{\mathrm{d} 3}+\sum_{\mathrm{n}=4}^{\mathbf{N}} i_{\mathrm{dn}} \\
& =I_{\mathrm{D}}+I_{\mathrm{d} 1} \cos (\omega t)+I_{\mathrm{d} 2} \cos (2 \omega t)+I_{\mathrm{d} 3} \cos (3 \omega t)+\sum_{\mathrm{n}=4}^{\mathbf{N}} I_{\mathrm{dn}} \cos (\mathrm{n} \omega t),
\end{aligned}
$$

where $I_{\mathrm{D}}$ is the DC current, $N$ is the harmonic order, $i_{\mathrm{dn}}$ is the RF current of the respective harmonic order, and $I_{\mathrm{dn}}$ is the peak drain current of the respective harmonic order. Because of the drain current of the lower MOSFET transistor is $180^{\circ}$ out of phase with respect to the upper drain current $\left(i_{\mathrm{D} 1}\right)$ 


$$
\begin{aligned}
i_{\mathrm{D} 2}= & i_{\mathrm{D} 1}\left(\omega t-180^{\circ}\right) \\
= & I_{\mathrm{D}}+I_{\mathrm{d} 1} \cos \left(\omega t-180^{\circ}\right)+I_{\mathrm{d} 2} \cos 2\left(\omega t-180^{\circ}\right)+I_{\mathrm{d} 3} \cos 3\left(\omega t-180^{\circ}\right) \\
& +\sum_{\mathrm{n}=4}^{\mathrm{N}} I_{\mathrm{dn}} \cos \mathrm{n}\left(\omega t-180^{\circ}\right) \\
= & I_{\mathrm{D}}-I_{\mathrm{d} 1} \cos (\omega t)+I_{\mathrm{d} 2} \cos 2(\omega t)-I_{\mathrm{d} 3} \cos 3(\omega t) \\
& +\sum_{\mathrm{n}=2}^{\mathrm{N} / 2} I_{\mathrm{d}(2 \mathrm{n})} \cos ((2 \mathrm{n}) \omega t)-\sum_{\mathrm{n}=3}^{(\mathrm{N}+1) / 2} I_{\mathrm{d}(2 \mathrm{n}-1)} \cos ((2 \mathrm{n}-1) \omega t) .
\end{aligned}
$$

Thus, the total drain current produced by push-pull amplifier becomes

$$
i_{\mathrm{D}}=i_{\mathrm{D} 1}-i_{\mathrm{D} 2}=2 I_{\mathrm{d} 1} \cos (\omega t)+2 I_{\mathrm{d} 3} \cos 3(\omega t)+\sum_{\mathrm{n}=3}^{(\mathrm{N}+1) / 2} 2 I_{\mathrm{d}(2 \mathrm{n}-1)} \cos ((2 \mathrm{n}-1) \omega t) .
$$

From equation 3.5, it is clear that the total drain current contains only the odd harmonics components after the cancellation of DC component and the even harmonics components. This reduces the distortion of the output signal.

\subsection{Specifications of RFPAs}

In order to fully characterize the amplifiers in MRI systems, it is necessary to analyse its performance in three domains: Time domain, Frequency domain, and Power domain.

\subsubsection{Specifications in Time Domain}

This class of specifications analyses the RF pulse waveform after the amplification process done by the RFPA. It studies several RF pulse parameters which can explain the distortion that appears by using a typical MRI RFPA. Fig. 3.7, shows the ideal pulse waveform produced by an ideal RFPA and the actual pulse waveform produced by non-ideal RFPA. Table 3.1 defines the actual RF pulse parameters. A brief explanation for these parameters will be given in the following [43]: 
- Bias enable, disable transient: This distortion appears as a voltage spike on the RF pulse due to the rapidly changing bias current when pulse operating mode is enabled.

- Un-Blanking, Blanking propagation delay time: During the MRI signal acquisition period, RFPAs are shut off to reduce any electrical noise that might be emitted. Turning the RFPA on and off requires a TTL signal that is synchronized with the RF pulse sequence. This signal is called un-blank signal which is applied to the RFPA though its bias voltage. The measure of an RFPA's ability to rapidly turn on and off is called un-blanking delay. In MRI, the maximum duration of this delay is 2 usec.

- Pulse pre-shoot, post-pulse backswing: This distortion appears as a superimposed half or more cycles of a low frequency signal on the un-blanked noise voltage. It occurs when the RFPA is un-blanked or the RF pulse is terminated.

- Rise, fall time: It is also known as transition duration. This defines two time intervals, the starting and the termination intervals of the RF pulse waveform. When the RF pulse is started, the time to transition from $10 \%$ to $90 \%$ of the voltage waveform is called rise time. On the contrary, when the RF pulse is terminated, the time to transition from $90 \%$ to $10 \%$ of the voltage waveform is called fall time. In MRI, these parameters are limited between $250 \mathrm{nsec}$ and 10 usec.

- Overshoot, rising/falling edge: This distortion appears as a voltage spike on the rising and falling edges of the RF pulse. It occurs due to rapid current change through inductors within the RFPA circuitry. In MRI, this parameter is allowed to extend up to $13 \%$.

- Pulse overshoot ringing/decay time: This distortion appears directly after the RF rise time as amplitude modulation of RF pulse. This modulation occurs by a low frequency, damped sinusoidal wave which is generated due to energy transfer between inductive and capacitive circuits in the RFPA. In MRI, this parameter is allowed to extend up to 5 usec.

- Pulse tilt (positive or negative): This distortion appears as a tilt on the RF pulse either positively (the amplitude increases with time) or negatively (the amplitude decreases with time). This occurs due to the increase in temperature of transistor which leads the gain of the RFPA to decrease over time (or increase for certain transistor types). In MRI, this parameter is allowed to extend up to $8 \%$ over a $20 \mathrm{msec}$ rectangular pulse. 

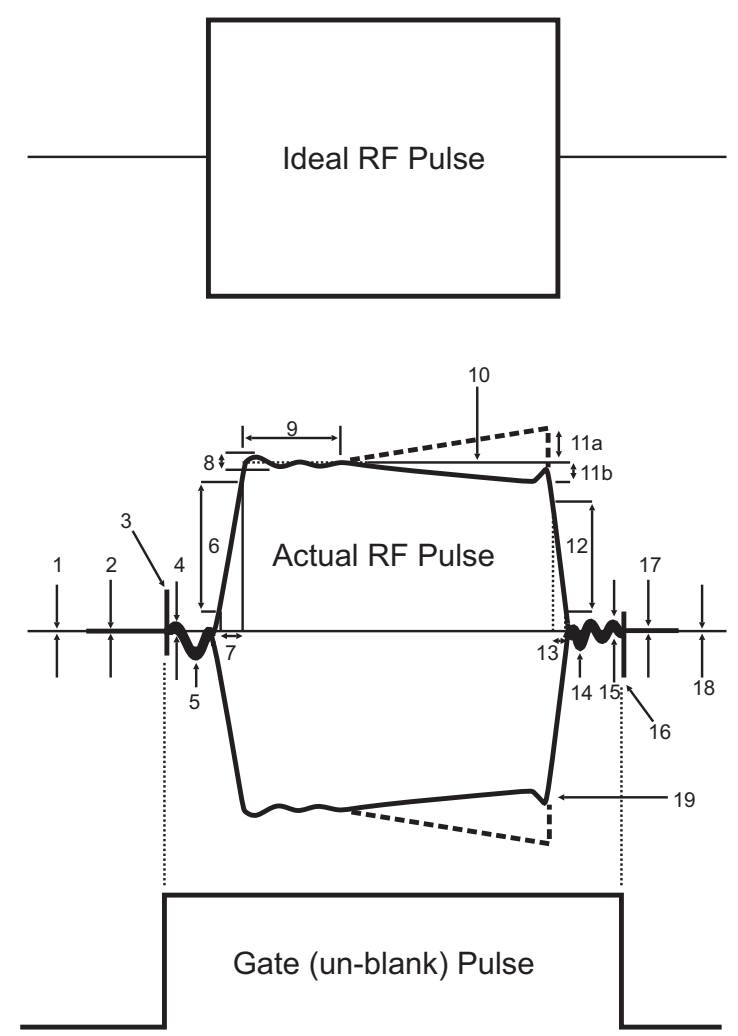

Figure 3.7: Ideal pulse waveform vs. actual pulse waveform. After [43]

- Long term amplitude/phase stability: This distortion appears as a change in amplitude and phase while amplifying a sequence of pulses which can be several minutes or hours. This occurs due to the influence of the RFPA gain and insertion phase by the temperature of the heat sink. In MRI, this parameter is allowed to extend up to $3 \%$ over a 24 hour period under constant ambient temperature.

- Phase error over-pulse: This distortion occurs due to the change in output power across the duration of rectangular RF pulse where the pulse tilt takes a place. This leads to a slight change in phase shift from the input of RFPA to the output causing AM-PM (Amplitude Modulation to Phase Modulation) distortion.

\subsubsection{Specifications in Frequency Domain}

This class of specifications analyses the distortion produced by real RFPAs over a wide range of frequencies. Since an ideal amplifier exhibits linear transfer characteristic, it amplifies the input signal with a scale value to produce the output signal without affecting the waveform of the signal. In other words, the output frequency spectrum of the amplifier is a perfect replica of the input frequency spectrum with larger amplitude, where no extra frequency components 
Table 3.1: Pulse parameter definitions. After [43]

\begin{tabular}{|c|c|c|c|}
\hline $\begin{array}{l}\text { Ref. in Fig. } 3.7 \\
\text { and Fig. 3.11 }\end{array}$ & Parameter name & Description & Dimension \\
\hline 1,18 & $\begin{array}{l}\text { Ideal, zero volts } \\
\text { reference line }\end{array}$ & $\begin{array}{l}\text { Mathematical construct, point of } \\
\text { reference for all pulse voltages }\end{array}$ & volts \\
\hline 2,17 & $\begin{array}{l}\text { Blanked noise } \\
\text { voltage }\end{array}$ & Noise out of RFPA when blanked & volts \\
\hline 3 & $\begin{array}{c}\text { Bias enable } \\
\text { transient voltage }\end{array}$ & $\begin{array}{l}\text { voltage spike exiting RFPA when RFPA is } \\
\text { initially un-blanked pre-RF pulse }\end{array}$ & volts \\
\hline 4,15 & $\begin{array}{l}\text { Un-blanked noise } \\
\text { voltage }\end{array}$ & $\begin{array}{c}\text { Noise voltage out of RFPA when } \\
\text { un-blanked }\end{array}$ & volts \\
\hline 5 & Pulse pre-shoot & $\begin{array}{c}\text { Amplifier output zero volt reference } \\
\text { deviation prior to pulse rise }\end{array}$ & $\%$ \\
\hline 6 & Pulse rising edge & $10-90 \%$ voltage transition range & volts \\
\hline 7 & $\begin{array}{l}\text { Pulse rise } \\
\text { transition } \\
\text { duration }\end{array}$ & $\begin{array}{l}\text { Time required for amplifier to transition } \\
\text { from } 10-90 \% \text { of peak output voltage }\end{array}$ & $\begin{array}{l}\text { micro/nano sec- } \\
\text { onds }\end{array}$ \\
\hline 8 & $\begin{array}{l}\text { Rising pulse } \\
\text { overshoot }\end{array}$ & $\begin{array}{c}\text { Portion RF pulse that exceeds } 100 \% \\
\text { amplitude during post rising transition } \\
\text { duration }\end{array}$ & $\%$ \\
\hline 9 & $\begin{array}{l}\text { Pulse overshoot } \\
\text { ringing/decay } \\
\text { time }\end{array}$ & $\begin{array}{l}\text { Time for damped sinusoidal envelope } \\
\text { peak to decay }\end{array}$ & $\begin{array}{l}\text { micro/nano sec- } \\
\text { onds }\end{array}$ \\
\hline 10 & $\begin{array}{l}100 \% \text { pulse RF } \\
\text { amplitude } \\
\text { reference }\end{array}$ & $\begin{array}{l}\text { Point on RF pulse that is chosen to be } 100 \\
\% \text {, usually after overshoot and ringing are } \\
\text { done }\end{array}$ & volts \\
\hline $11 \mathrm{a}, \mathrm{b}$ & $\begin{array}{c}\text { Pulse tilt } \\
\text { positive/negative }\end{array}$ & $\begin{array}{l}\text { Amount that peak RF voltage slopes } \\
\text { throughout pulse width duration }\end{array}$ & $\%$ \\
\hline 12 & Pulse falling edge & $90-10 \%$ voltage transition range & volts \\
\hline 13 & $\begin{array}{l}\text { Pulse falling } \\
\text { transition } \\
\text { duration }\end{array}$ & $\begin{array}{l}\text { Time required for amplifier to transition } \\
\text { from } 90-10 \% \text { of peak output voltage }\end{array}$ & $\begin{array}{l}\text { micro/nano sec- } \\
\quad \text { onds }\end{array}$ \\
\hline 14 & $\begin{array}{l}\text { Post pulse } \\
\text { backswing }\end{array}$ & $\begin{array}{l}\text { Amplifier output zero volt reference } \\
\text { deviation after pulse fall time }\end{array}$ & $\%$ \\
\hline 16 & $\begin{array}{l}\text { Bias disable } \\
\text { transient }\end{array}$ & $\begin{array}{l}\text { Voltage spike exiting RFPA when RFPA } \\
\text { is blanked post-RF pulse }\end{array}$ & volts \\
\hline 19 & $\begin{array}{l}\text { Falling pulse } \\
\text { overshoot }\end{array}$ & $\begin{array}{l}\text { Portion of RF pulse that exceeds final tilt } \\
\text { amplitude pre-falling transition duration }\end{array}$ & $\%$ \\
\hline 20 & $\begin{array}{l}\text { AM-AM } \\
\text { distortion }\end{array}$ & $\begin{array}{l}\text { Inter-pulse amplitude ratio distortion, also } \\
\text { known as gain linearity }\end{array}$ & $\mathrm{db}$ \\
\hline 21 & $\begin{array}{l}\text { AM-PM } \\
\text { distortion }\end{array}$ & $\begin{array}{l}\text { Inter-pulse relative phase distortion, also } \\
\text { known as phase linearity }\end{array}$ & degrees \\
\hline 22 & $\begin{array}{l}\text { Amplitude } \\
\text { stability }\end{array}$ & Long term gain stability over time & db/hours \\
\hline 23 & Phase stability & Long term phase stability over time & degrees/hours \\
\hline 24 & $\begin{array}{l}\text { Phase-error, } \\
\text { over-pulse }\end{array}$ & $\begin{array}{l}\text { Phase shift across the duration of a pulse } \\
\text { width }\end{array}$ & degrees \\
\hline
\end{tabular}


have been introduced. In reality, all RFPAs exhibit a non-linear transfer characteristic, i.e., the amplification factor is a function of signal level. It can be described by a complex transfer function containing non-linear terms. In the MRI application, we usually apply only single RF tones (sinusoidal signals); therefor, in the following, the discussion of nonlinearity effects will be limited to this situation. By applying a sinusoidal input signal to an amplifier, we will get an output signal with a waveform that is non-identical (distorted) to the input one and the output frequency spectrum will contain extra frequency components (integer multiples) beside the input frequency. These frequency components (harmonic frequencies) are known as Harmonic Distortion. To characterize the non-linear behaviour of RFPAs, a non-linear mathematical model is needed. Polynomial approximation containing non-linear terms based upon Taylor series expansion is considered an appropriate description for "soft" non-linearity. [50]

The Taylor series expansion which models the non-linearity of RFPAs can be written as

$$
y(t)=y_{0}+a_{1} x(t)+a_{2} x^{2}(t)+a_{3} x^{3}(t)+\ldots+a_{\mathrm{N}} x^{\mathrm{N}}(t)=y_{0}+\sum_{\mathrm{n}=1}^{\mathrm{N}} a_{\mathrm{n}} x^{\mathrm{n}}(t)
$$

where $y(t)$ is the output signal, $y_{0}$ is the output operating point (DC bias voltage), $x(t)$ is the input signal and $a_{\mathrm{i}}$ are the coefficients of the Taylor series.

To understand the harmonic distortion more precisely, we need to study the higher order components that are introduced in the output frequency spectrum in more details.

\section{Second-Order Nonlinearity}

A Taylor series of 2nd order can describe the simplest non-linearity of amplitude by adding a squared term to the ideal transfer characteristic. And by neglecting the DC bias $y_{0}=0$, the input-output relationship will be written as

$$
y(t)=G_{1} x(t)+G_{2} x^{2}(t)
$$

where $G_{1}$ is the linear gain and $G_{2}$ is the gain of the quadratic term. Now, by applying a single-tone RF input signal

$$
x(t)=A_{1} \sin \left(\omega_{0} t\right)
$$




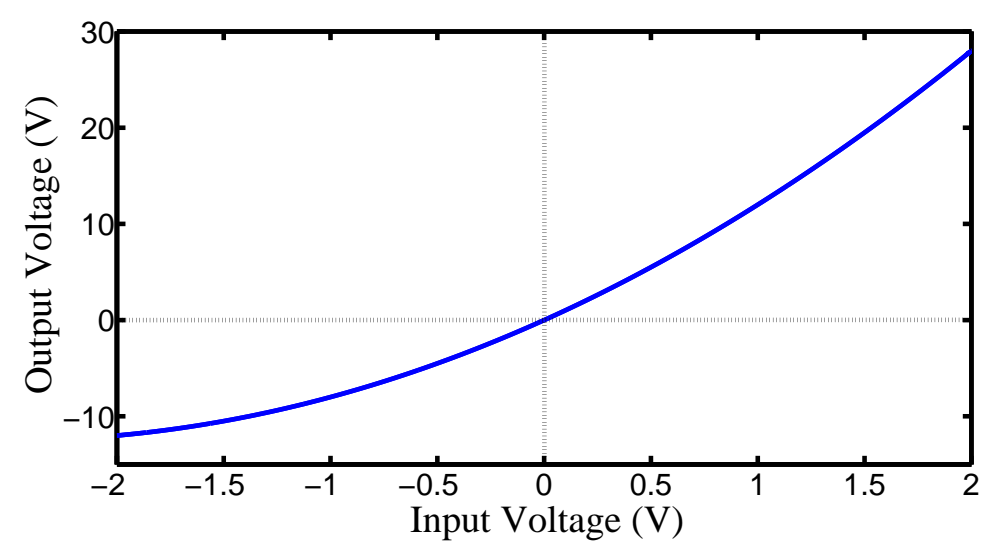

(a)
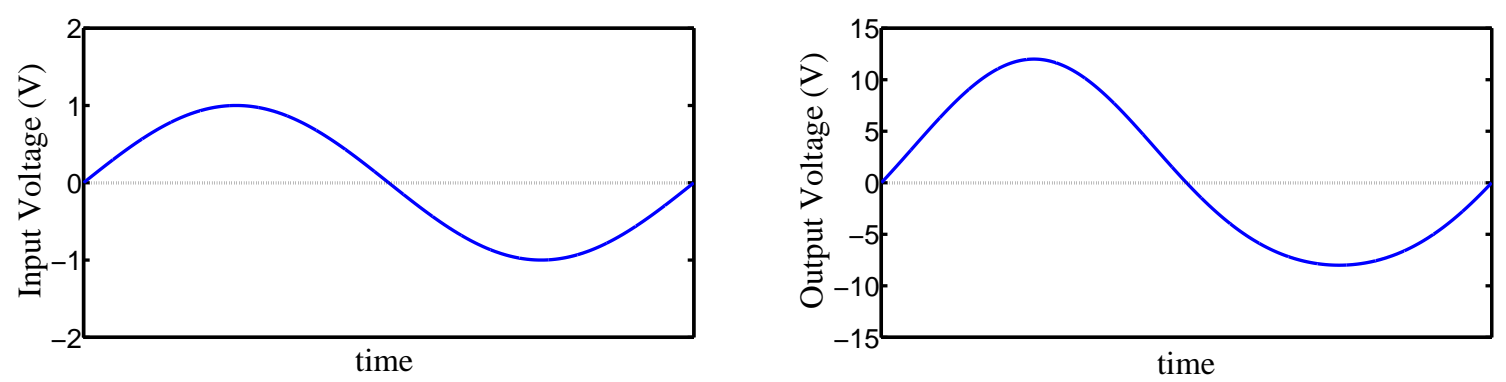

(b)
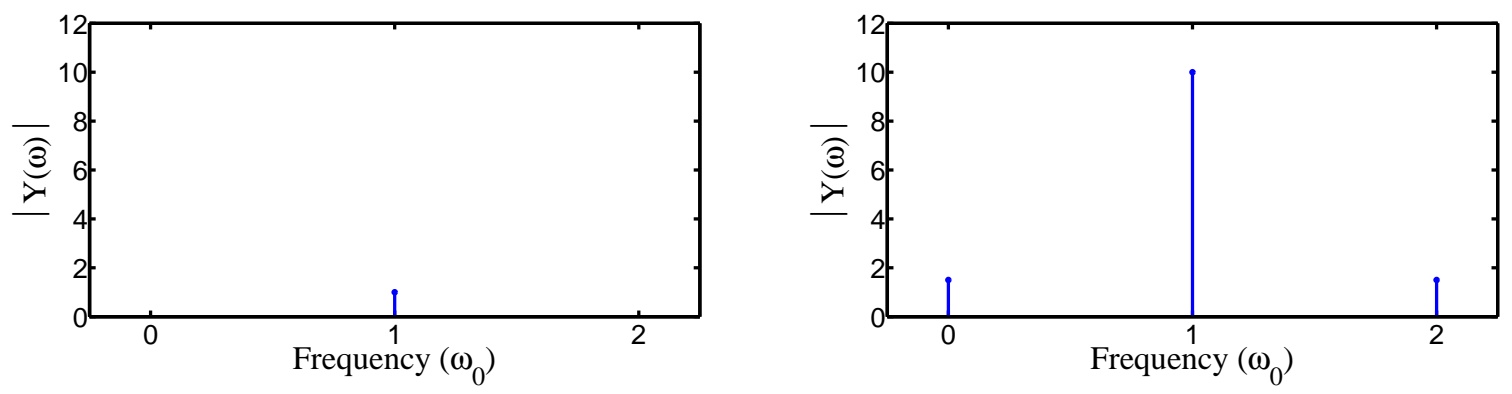

(c)

Figure 3.8: Second order nonlinearity. (a) Second order transfer characteristic, (b) time domain characteristic, (c) frequency domain characteristic.

to the second-order transfer characteristic, the input-output relationship becomes

$$
\begin{aligned}
y(t) & =G_{1} A_{1} \sin \left(\omega_{0} t\right)+G_{2} A_{1}^{2} \sin ^{2}\left(\omega_{0} t\right) \\
& =G_{1} A_{1} \sin \left(\omega_{0} t\right)+G_{2} A_{1}^{2} \frac{1-\cos \left(2 \omega_{0} t\right)}{2} \\
& =\underbrace{\frac{1}{2} G_{2} A_{1}^{2}}_{\text {DC component }}+\underbrace{G_{1} A_{1} \sin \left(\omega_{0} t\right)}_{\text {fundamental component }}-\underbrace{\frac{1}{2} G_{2} A_{1}^{2} \cos \left(2 \omega_{0} t\right)}_{\text {second harmonic }},
\end{aligned}
$$

where $A_{1}$ is the amplitude of the input signal. From Equation (3.9), we notice that the output 
signal consists of three terms: a DC component, the fundamental component, and a new component whose frequency is twice the fundamental frequency. Fig. (3.8) shows an example that demonstrates the effect of the second-order non-linear characteristic on a pure sinusoidal input signal for the case where $A_{1}=1 V, G_{1}=10$, and $G_{2}=3$. The distortion appears due to the curvature in the transfer characteristic which compresses the excursion of the output signal on the negative side. By looking at the output frequency spectrum, we can notice that this distortion produces a second harmonic component but has no influence on the fundamental component. Such "out-of-band" distortion can be removed by band-pass or low-pass filtering. [50]

\section{Third-Order Nonlinearity}

The third order non-linearity adds a third-order term to the ideal transfer characteristic and creates a different type of distortion. By considering $y_{0}=0$, the input-output relationship will be written as

$$
y(t)=G_{1} x(t)+G_{3} x^{3}(t)
$$

where $G_{3}$ is the gain of the cubic term. Now, by applying a single-tone RF input signal to the third-order transfer characteristic, the input-output relationship becomes

$$
\begin{aligned}
y(t) & =G_{1} A_{1} \sin \left(\omega_{0} t\right)+G_{3} A_{1}^{3} \sin ^{3}\left(\omega_{0} t\right) \\
& =G_{1} A_{1} \sin \left(\omega_{0} t\right)+G_{3} A_{1}^{3}\left[\frac{3}{4} \sin \left(\omega_{0} t\right)-\frac{1}{4} \sin \left(3 \omega_{0} t\right)\right] \\
& =\underbrace{\left(G_{1} A_{1}+\frac{3}{4} G_{3} A_{1}^{3}\right) \sin \left(\omega_{0} t\right)}_{\text {fundamental component }}-\underbrace{\frac{1}{4} G_{3} A_{1}^{3} \sin \left(3 \omega_{0} t\right)}_{\text {third harmonic }} .
\end{aligned}
$$

From Equation 3.11, we notice that the output signal has a new component whose frequency is three times the fundamental frequency. No DC component is generated contrary to the second order non-linearity. But instead, a new term which is proportional to the cube of the input amplitude has been superimposed onto the fundamental component. Such in-band distortion cannot be removed by filtering. Fig. 3.9 shows an example of the third-order non-linearity characteristic for the case where $A_{1}=1 \mathrm{~V}, G_{1}=10$ and $G_{3}=-3$. From the output waveform in time domain, we recognize that the distortion creates a soft-clipped signal due to a saturation 


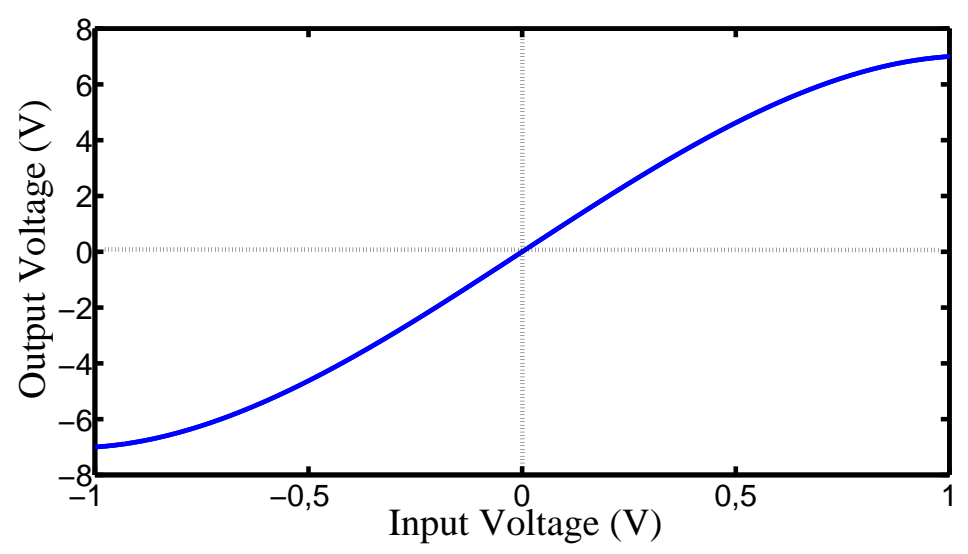

(a)
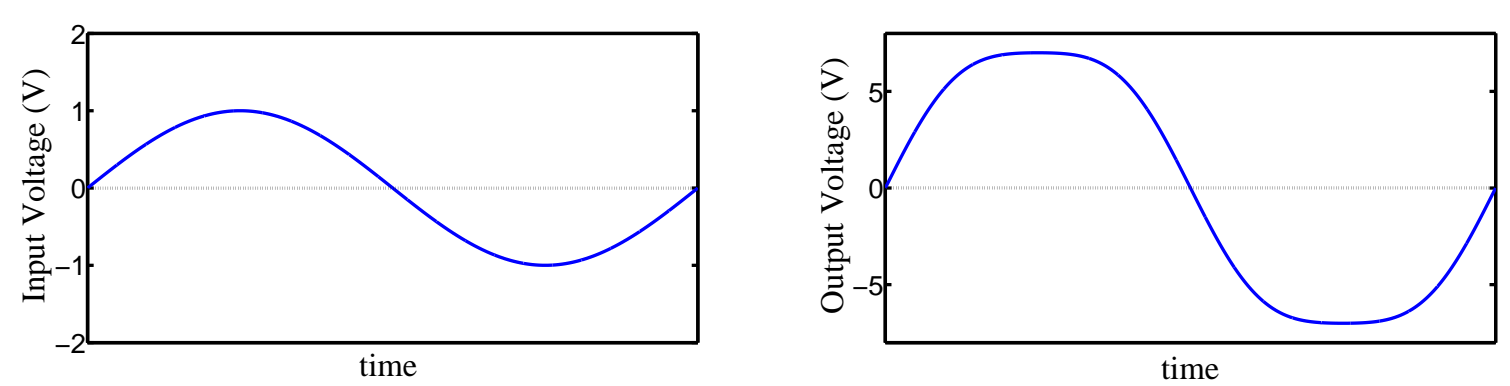

(b)
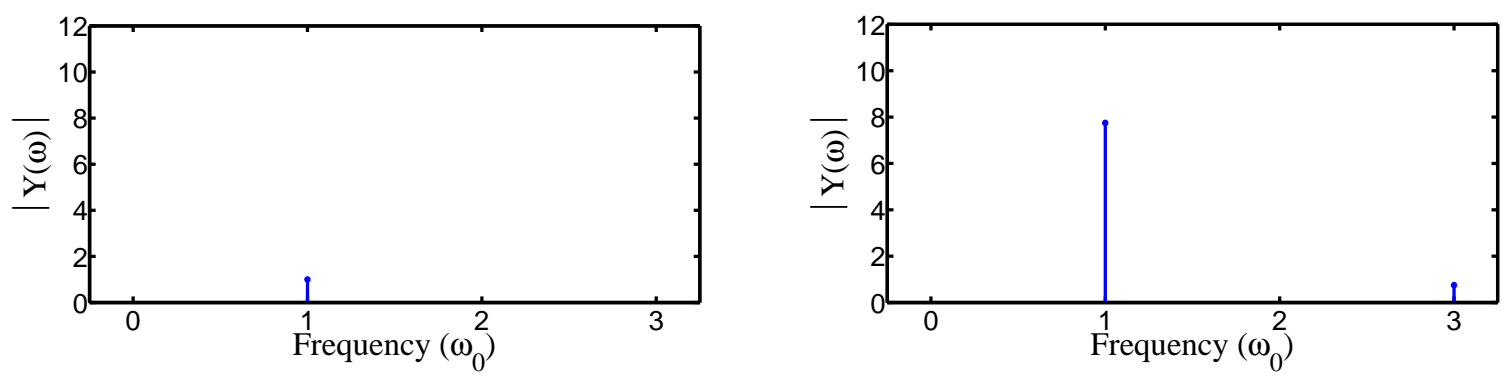

(c)

Figure 3.9: Third order nonlinearity. (a) Third order transfer characteristic, (b) time domain characteristic, (c) frequency domain characteristic.

effect in the transfer characteristic. The frequency spectrum shows a gain compression at the fundamental frequency due to the in-band distortion.

\section{Higher-Order Nonlinearities}

In order to generalize the non-linear transfer characteristic of the RFPA more precisely, we need to derive a non-linear model containing all the components that contribute in the distortion of the fundamental component. This can be done by analysing the nth-order non-linearity in terms of 
even-order and odd-order non-linearity.

\section{- Even-order Nonlinearity}

The input-output relationship by considering the nth (even)-order non-linearity can be characterized by

$$
y(t)=\sum_{\mathrm{n}=1}^{\mathrm{N} / 2} G_{2 \mathrm{n}} x^{2 \mathrm{n}}(t) .
$$

Now, by applying a single-tone RF input signal from Equation (3.8), the output becomes

$$
y(t)=\sum_{\mathrm{n}=1}^{\mathbf{N} / 2} G_{2 \mathrm{n}} A_{1}^{2 \mathrm{n}} \sin ^{2 \mathrm{n}}\left(\omega_{0} t\right)
$$

By benefiting from the trigonometric power formulas in [51], the sine term can be expanded as the following

$$
\sin ^{2 \mathrm{n}} x=\frac{1}{2^{2 \mathrm{n}}}\left(\begin{array}{c}
2 \mathrm{n} \\
\mathrm{n}
\end{array}\right)+\frac{(-1)^{\mathrm{n}}}{2^{2 \mathrm{n}-1}} \sum_{\mathrm{k}=0}^{\mathrm{n}-1}(-1)^{\mathrm{k}}\left(\begin{array}{c}
2 \mathrm{n} \\
\mathrm{k}
\end{array}\right) \cos [2(\mathrm{n}-\mathrm{k}) x]
$$

Thus, the general formulation of the nth (even)-order non-linearity can be written as

$$
y(t)=\underbrace{\sum_{\mathrm{n}=1}^{\mathrm{N} / 2}\left(\begin{array}{c}
2 \mathrm{n} \\
n
\end{array}\right) \frac{G_{2 \mathrm{n}} A_{1}^{2 \mathrm{n}}}{2^{2 \mathrm{n}}}}_{\text {DC component }}+\underbrace{\sum_{\mathrm{n}=1}^{\mathbf{N} / 2} \frac{(-1)^{\mathrm{n}} G_{2 \mathrm{n}} A_{1}^{2 \mathrm{n}}}{2^{2 \mathrm{n}-1}} \sum_{\mathrm{k}=0}^{\mathrm{n}-1}(-1)^{\mathrm{k}}\left(\begin{array}{c}
2 \mathrm{n} \\
\mathrm{k}
\end{array}\right) \cos \left[2(\mathrm{n}-\mathrm{k}) \omega_{0} t\right]}_{\text {even harmonics }} .
$$

From Equation (3.15), we conclude that an even-order non-linearity has no influence on the fundamental component. It produces DC components in addition to the harmonic components at even multiples of the fundamental frequency. 


\section{- Odd-order Nonlinearity}

For the nth (odd)-order non-linearity, the input-output relationship can be characterized by

$$
y(t)=\sum_{\mathrm{n}=1}^{(\mathrm{N}+1) / 2} G_{2 \mathrm{n}-1} x^{2 \mathrm{n}-1}(t) .
$$

Now, by applying a single-tone RF input signal from Equation (3.8), the output becomes

$$
y(t)=\sum_{\mathrm{n}=1}^{(\mathrm{N}+1) / 2} G_{2 \mathrm{n}-1} A_{1}^{2 \mathrm{n}-1} \sin ^{2 \mathrm{n}-1}\left(\omega_{0} t\right)
$$

From the trigonometric power formulas in [51], the sine term can be expanding as the following

$$
\sin ^{2 \mathrm{n}-1} x=\frac{1}{2^{2 \mathrm{n}-2}} \sum_{\mathrm{k}=0}^{\mathrm{n}-1}(-1)^{\mathrm{n}-\mathrm{k}-1}\left(\begin{array}{c}
2 \mathrm{n}-1 \\
\mathrm{k}
\end{array}\right) \sin [(2(\mathrm{n}-\mathrm{k})-1) x]
$$

The general formulation of the nth (odd)-order non-linearity can be written as

$$
\begin{aligned}
y(t)= & \sum_{\mathrm{n}=1}^{(\mathrm{N}+1) / 2} \frac{G_{2 \mathrm{n}-1} A_{1}^{2 \mathrm{n}-1}}{2^{2 \mathrm{n}-2}} \sum_{\mathrm{k}=0}^{\mathrm{n}-1}(-1)^{\mathrm{n}-\mathrm{k}-1}\left(\begin{array}{c}
2 \mathrm{n}-1 \\
\mathrm{k}
\end{array}\right) \sin \left[(2(\mathrm{n}-\mathrm{k})-1)\left(\omega_{0} t\right)\right] \\
= & \underbrace{\sum_{\mathrm{n}=1}^{(\mathrm{N}+1) / 2} \frac{G_{2 \mathrm{n}-1} A_{1}^{2 \mathrm{n}-1}}{2^{2 \mathrm{n}-2}} \sum_{\mathrm{k}=0}^{\mathrm{n}-1}(-1)^{\mathrm{n}-\mathrm{k}-1}\left(\begin{array}{c}
2 \mathrm{n}-1 \\
\mathrm{k}
\end{array}\right) \sin \left(\omega_{0} t\right)}_{\text {fundamental component }} \\
& +\underbrace{\sum_{\mathrm{n}=2}^{(\mathrm{N}+1) / 2} \frac{G_{2 \mathrm{n}-1} A_{1}^{2 \mathrm{n}-1}}{2^{2 \mathrm{n}-2}} \sum_{\mathrm{k}=0}^{\mathrm{n}-2}(-1)^{\mathrm{n}-\mathrm{k}-1}\left(\begin{array}{c}
2 \mathrm{n}-1 \\
\mathrm{k}
\end{array}\right) \sin \left[(2(\mathrm{n}-\mathrm{k})-1)\left(\omega_{0} t\right)\right]}_{\text {odd harmonics }} .
\end{aligned}
$$

From Equation (3.19), we conclude that an odd-order non-linearity produces odd-order harmonics in addition to a fundamental component. 


\section{- Total Distortion}

The general formulation of the nth-order non-linearity can be obtained by adding Equation (3.15) and (3.19) together with the DC bias in Equation (3.6)

$$
\begin{aligned}
& y(t)=\underbrace{y_{0}+\sum_{\mathrm{n}=1}^{\mathbf{N} / 2}\left(\begin{array}{c}
2 \mathrm{n} \\
\mathrm{n}
\end{array}\right) \frac{G_{2 \mathrm{n}} A_{1}^{2 \mathrm{n}}}{2^{2 \mathrm{n}}}}_{\text {DC component }} \\
& +\underbrace{\sum_{\mathrm{n}=1}^{(\mathrm{N}+1) / 2} \frac{G_{2 \mathrm{n}-1} A_{1}^{2 \mathrm{n}-1}}{2^{2 \mathrm{n}-2}} \sum_{\mathrm{k}=0}^{\mathrm{n}-1}(-1)^{\mathrm{n}-\mathrm{k}-1}\left(\begin{array}{c}
2 \mathrm{n}-1 \\
\mathrm{k}
\end{array}\right) \sin \left(\omega_{0} t\right)}_{\text {fundamental component }} \\
& +\underbrace{\sum_{\mathrm{n}=1}^{\mathrm{N} / 2} \frac{(-1)^{\mathrm{n}} G_{2 \mathrm{n}} A_{1}^{2 \mathrm{n}}}{2^{2 \mathrm{n}-1}} \sum_{\mathrm{k}=0}^{\mathrm{n}-1}(-1)^{\mathrm{k}}\left(\begin{array}{c}
2 \mathrm{n} \\
\mathrm{k}
\end{array}\right) \cos \left[2(\mathrm{n}-\mathrm{k}) \omega_{0} t\right]}_{\text {even harmonics }} \\
& +\underbrace{\sum_{\mathrm{n}=2}^{(\mathrm{N}+1) / 2} \frac{G_{2 \mathrm{n}-1} A_{1}^{2 \mathrm{n}-1}}{2^{2 \mathrm{n}-2}} \sum_{\mathrm{k}=0}^{\mathrm{n}-2}(-1)^{\mathrm{n}-\mathrm{k}-1}\left(\begin{array}{c}
2 \mathrm{n}-1 \\
\mathrm{k}
\end{array}\right) \sin \left[(2(\mathrm{n}-\mathrm{k})-1)\left(\omega_{0} t\right)\right]}_{\text {odd harmonics }} .
\end{aligned}
$$

In an MRI system, the usual limitation of even order harmonics is $-20 \mathrm{dbc}$, and for odd order harmonics it is $-12 \mathrm{dbc}$. This rather "loose" limit is acceptable because the MR-coils are resonant circuits which act as band-pass filters.

\subsubsection{Specifications in Power Domain}

This class of specifications characterizes the output power of the RFPA when the input power is swept across a certain dynamic range. It characterizes the non-linearity of the RFPA in the form of Amplitude-to-Amplitude conversion (AM/AM) and Amplitude-to-Phase (AM/PM) conversion as seen in Fig. (3.10). These two response parameters describe the amplifier gain and phase as a function of the input power. 


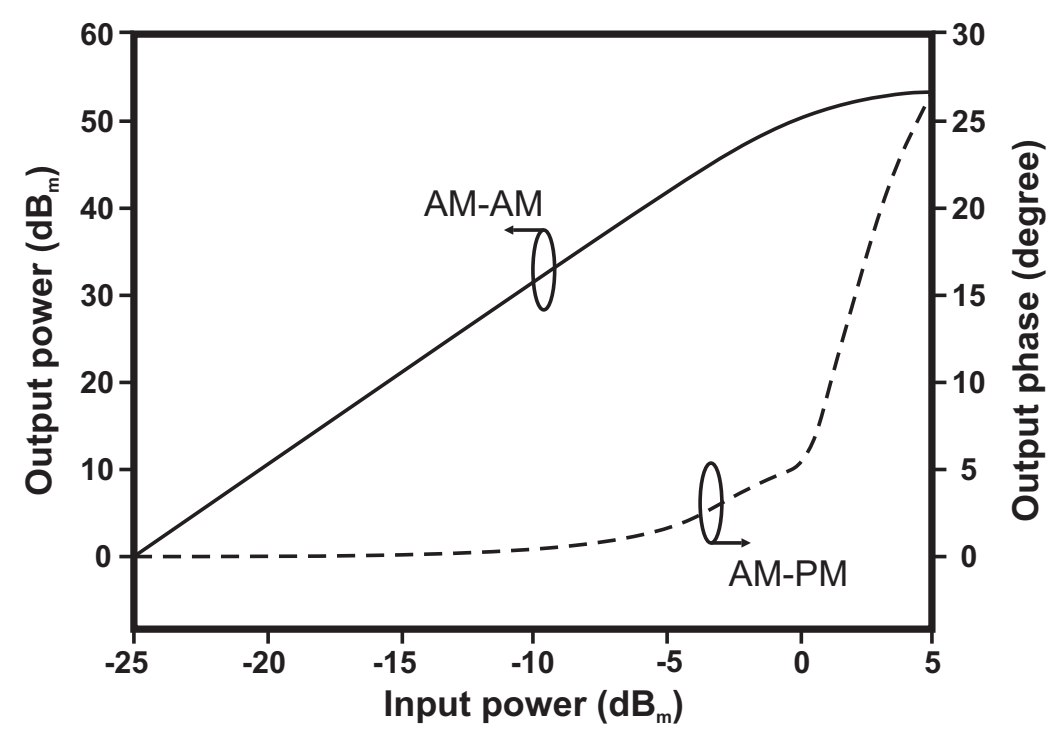

Figure 3.10: Typical (AM/AM) and (AM/PM) curves.

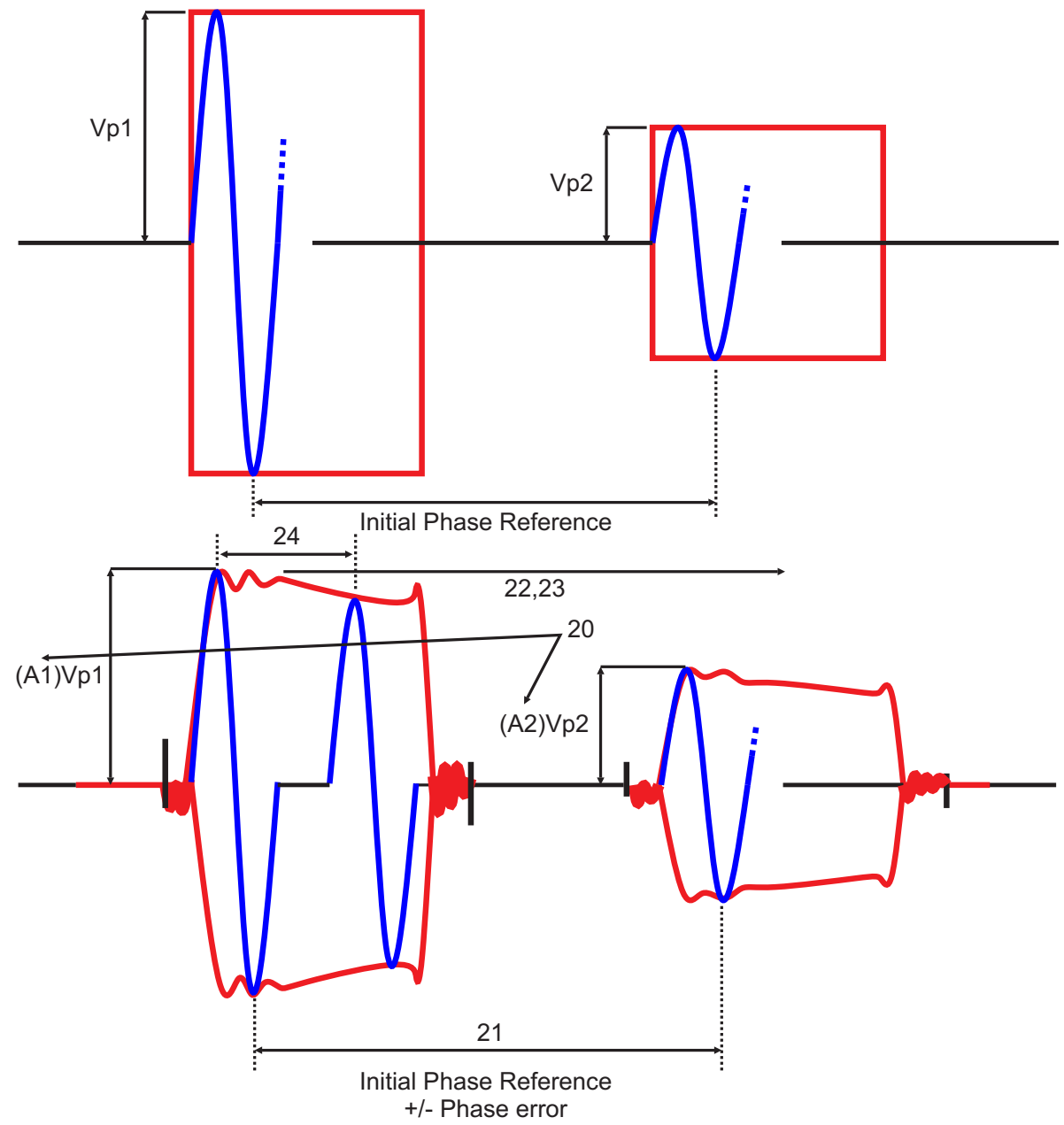

Figure 3.11: Representation of gain distortion and phase distortion in a given pulse sequence. After [43] 


\section{Gain Linearity}

Gain linearity is defined as the ability of an amplifier to hold its gain constant throughout the application of an RF input signal with varying power levels [52]. An ideal linear amplifier has linear transfer relationship between the input and output power over all power levels, while an actual amplifier has power gain which is input power level dependent. Fig. (3.11) shows different power gain factors $A_{1}$ and $A_{2}$ for different pulse amplitudes. In MRI systems, the allowable gain variation is $\pm 1 \mathrm{~dB}$ over $40 \mathrm{~dB}$ dynamic power range [43].

\section{Phase Linearity}

Phase linearity is defined as the ability for an RFPA to hold its insertion phase constant over varying output power levels [52], where the insertion phase is a relative phase added by the amplifier to the output signal and causing a relative phase shift between the input and output. For an ideal linear amplifier, the insertion phase remains constant throughout the amplification process over the dynamic range. Fig. (3.11) shows how the insertion phase changes between low power pulse and high power pulse with respect to the ideal case. In MRI systems, the allowable insertion phase variation is $\pm 7.5^{\circ}$ over $40 \mathrm{~dB}$ dynamic power range [43]. 


\subsection{Near-Magnet Power Amplifier}

\subsubsection{Introduction}

As mentioned in section 2.2.5, MRI systems with high magnetic field strength need to employ transmitters that have a capability to produce relatively high RF power. In 7 Tesla MRI systems, parallel RF transmission using multiple RF chains and multiple transmit elements is employed, where each channel is excited by an RFPA of $1 \mathrm{~kW}$ output power. This will offer several thousand watts which might be needed to be applied to the patient's body. But unfortunately a considerable portion of power is dissipated in transmit cables extended between the scanner and the technical room where the power amplifiers normally is placed. In order to decrease the cable length (i.e., the cable loss), a near-magnet power amplifier has been proposed [53] to be placed inside the scanner room in a rack behind the scanner. Such power amplifiers have to be built using non-magnetic components to maintain its functional characteristics when placed in the high magnetic stray field environment close to the magnet; this prohibits the use of circulators at the output stage of RFPAs which leads to load-dependency of the amplifier characteristics, e.g., of the output power from the RFPA.

\subsubsection{Power Amplifier with Built-in Coil Current Sensing Concept}

The near-magnet power amplifier that has been proposed in [53] employs a new concept of coil current sensing unlike concepts in [12,13] which use a current sensor integrated in a transmit coil to measure the current flows through the coil that is responsible to generate the $B_{1}$ field. The new concept with a special combination of the PA and the coil is shown in Fig 3.12. At the end of the output matching stage of the PA, two probes are used to sample the output voltage across a quarter-wave $(\lambda / 4)$ transmission line [54]. A meander dipole coil utilizes a quarter-wave $(\lambda / 4)$ transformer as a matching network which matches the low input impedance of the coil to the $50 \Omega$ coaxial impedance. The PA output is connected to the coil by a multiple of half-wave length $(N \times \lambda / 2)$ cable. Based on the current forcing property of a quarter wave lossless transmission line, the current $I_{2}$ into the coil is independent of the load impedance proportional to the voltage $U_{1}$ at the transformer primary side. To prove that, we need the following voltage and current waves on a lossless transmission line shown in Fig 3.13 

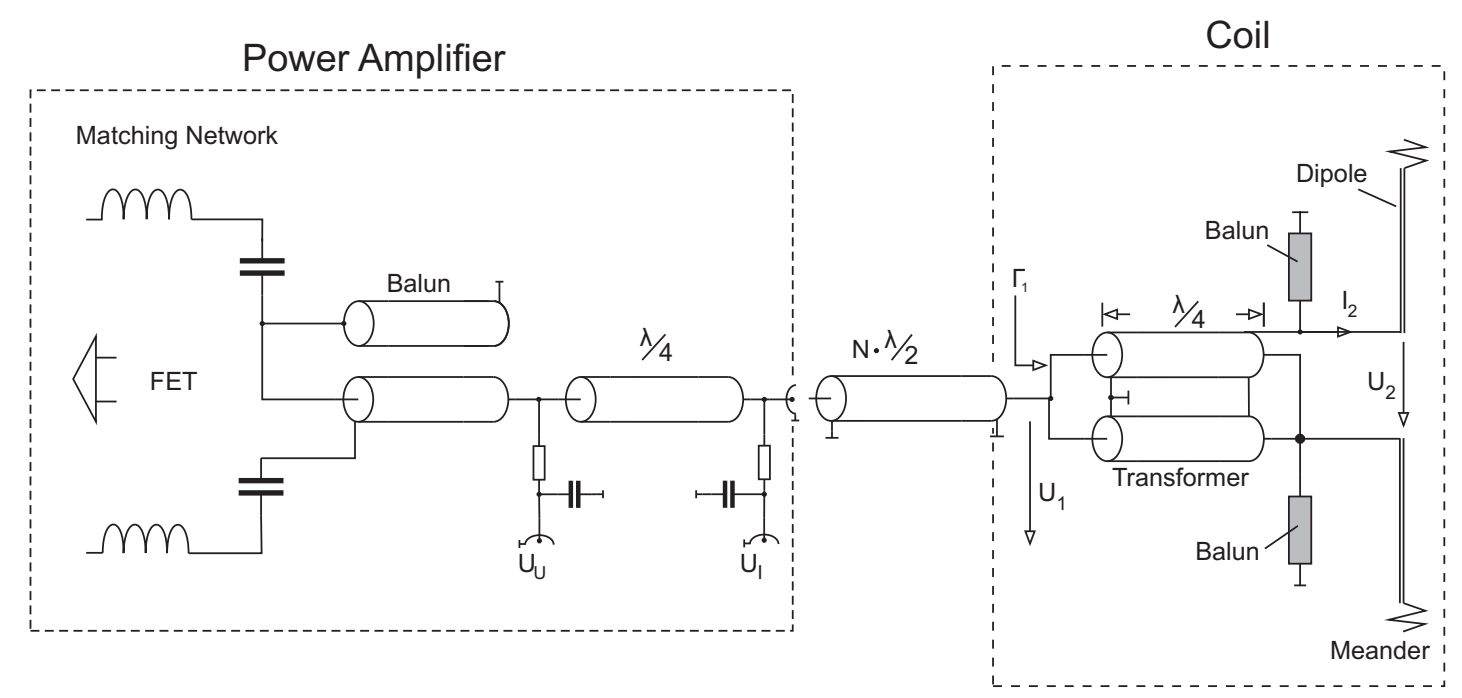

Figure 3.12: Circuit diagram for the new concept of power amplifier with built-in coil current sensing.

$$
\begin{aligned}
U(z) & =U^{+}(z)+U^{-}(z)=U_{\mathrm{o}}^{+} e^{-\mathrm{j} \beta z}+U_{\mathrm{o}}^{-} e^{\mathrm{j} \beta z} \\
I(z) & =I^{+}(z)+I^{-}(z)=I_{\mathrm{o}}^{+} e^{-\mathrm{j} \beta z}+I_{\mathrm{o}}^{-} e^{\mathrm{j} \beta z}
\end{aligned}
$$

where $U^{+}(z)$ and $I^{+}(z)$ are the forward travelling waves, $U^{-}(z)$ and $I^{-}(z)$ are the backward travelling waves, $U_{\mathrm{o}}^{+}$and $I_{\mathrm{o}}^{+}$are the wave amplitudes in the forward direction, $U_{\mathrm{o}}^{-}$and $I_{\mathrm{o}}^{-}$are the wave amplitudes in the backward direction, $e^{-\mathrm{j} \beta z}$ is the wave propagation in the $+z$ direction and $e^{\mathrm{j} \beta z}$ is the wave propagation in the $-z$ direction.

To find $U_{\mathrm{o}}^{+}$and $U_{\mathrm{o}}^{-}$, the terminal conditions at the load $(z=0)$ are required, say $U_{2}=U(z=0)$ and $I_{2}=I(z=0)$. Substituting these in Eq. 3.21 and using the telegrapher equation to relate

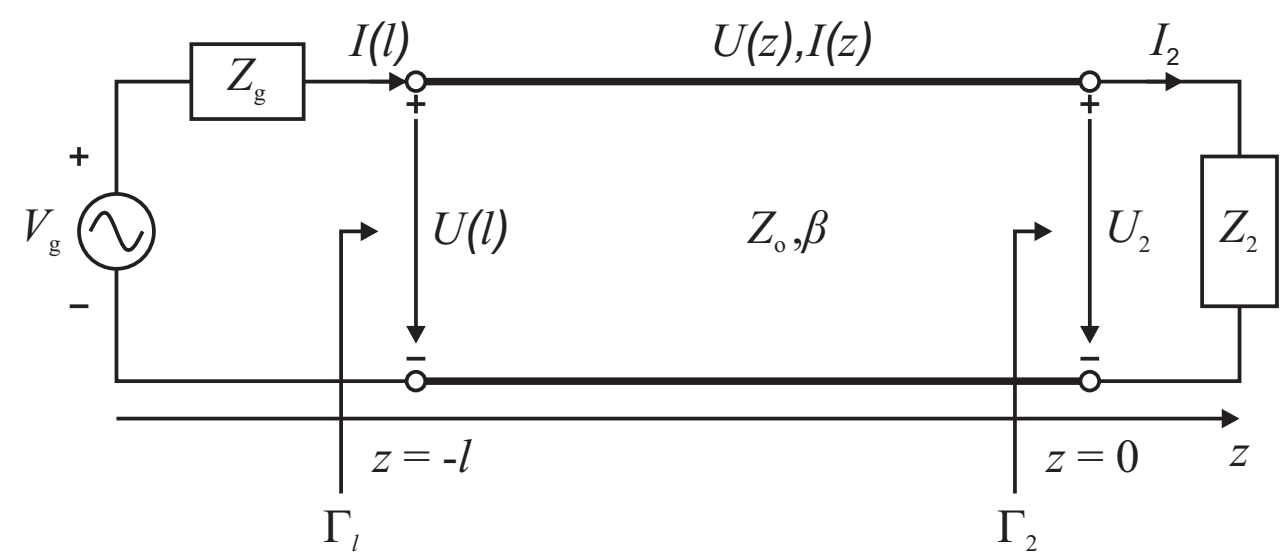

Figure 3.13: Voltage and current definitions for a lossless transmission line of length $l$, terminated by a load $Z_{2}$. 
the currents to the voltages of the waves results in

$$
\begin{gathered}
U_{2}=U_{\mathrm{o}}^{+}+U_{\mathrm{o}}^{-} \\
I_{2}=I_{\mathrm{o}}^{+}+I_{\mathrm{o}}^{-}=\frac{U_{\mathrm{o}}^{+}}{Z_{\mathrm{o}}}-\frac{U_{\mathrm{o}}^{-}}{Z_{\mathrm{o}}},
\end{gathered}
$$

where $Z_{\mathrm{o}}$ is the characteristic impedance of the transmission line which relates the voltage and current on the line as

$$
Z_{\mathrm{o}}=\frac{U_{\mathrm{o}}^{+}}{I_{\mathrm{o}}^{+}}=\frac{-U_{\mathrm{o}}^{-}}{I_{\mathrm{o}}^{-}}
$$

Rearranging the voltage and current at the load in Eq. 3.22, we get

$$
\begin{aligned}
U_{\mathrm{o}}^{+} & =\frac{1}{2} I_{2}\left(Z_{2}+Z_{\mathrm{o}}\right) \\
U_{\mathrm{o}}^{-} & =\frac{1}{2} I_{2}\left(Z_{2}-Z_{\mathrm{o}}\right)
\end{aligned}
$$

where $Z_{2}=U_{2} / I_{2}$. By substituting the expressions for $U_{\mathrm{o}}^{+}$and $U_{\mathrm{o}}^{-}$into the voltage wave in Eq. 3.21 at $z=-l$, we get

$$
\begin{aligned}
U(z=-l) & =\frac{1}{2} I_{2}\left[Z_{2}\left(e^{\mathrm{j} \beta l}+e^{-\mathrm{j} \beta l}\right)+Z_{\mathrm{o}}\left(e^{\mathrm{j} \beta l}-e^{-\mathrm{j} \beta l}\right)\right] \\
& =I_{2}\left[Z_{2} \cos (\beta l)+\mathrm{j} Z_{\mathrm{o}} \sin (\beta l)\right]
\end{aligned}
$$

For transmission line with quarter-wave length $(l=\lambda / 4)$, the voltage wave equation becomes

$$
U\left(l=\frac{\lambda}{4}\right)=\mathrm{j} I_{2} Z_{\mathrm{o}}
$$

By rearranging the Eq. 3.26, the load current can be expressed as 


$$
I_{2}=\frac{U\left(l=\frac{\lambda}{4}\right)}{Z_{\mathrm{o}}} \angle-90^{\circ}
$$

Using the load impedance $\left(Z_{2}=\frac{U_{2}}{I_{2}}\right)$, Eq. 3.27 in terms of output voltage $\left(U_{2}\right)$ becomes

$$
U_{2}=\frac{-\mathrm{j} Z_{2} U\left(l=\frac{\lambda}{4}\right)}{Z_{\mathrm{o}}} .
$$

Eq. 3.27 shows the mathematical definition of the current forcing property. Applying that in Fig. 3.12, we realize the proportionality between the coil current $I_{2}$ and the voltage $U_{1}$ at the other end of the transformer. The probe voltage $U_{\mathrm{I}}$ at the PA output is equal to this voltage if we assume that the connecting cable of length $N \cdot \lambda / 2$ is lossless. Thus, the probe voltage $U_{\mathrm{I}}$ is proportional to the coil current $I_{2}$. Furthermore, the other probe voltage $U_{\mathrm{U}}$ is proportional to the voltage appearing at the coil terminals $U_{2}$. The characteristic impedance of the quarter-wave transformer and the probe factor of the probes have direct influence on both proportionality constants. The probe voltage $U_{\mathrm{I}}$ cancels the need for an extra field sensor, as it can be used to control the coil current in case a Cartesian feedback loop is utilized (see Ch.4 and Ch.5).

\section{Quarter-wave lossy transmission line}

In practice, real transmission lines are lossy due to finite conductivity of the conductors and the lossy dielectric material between them. Signals propagating through lossy lines will be attenuated based on the attenuation constant $(\alpha)$ of these lines. Thus, the propagation constant $(\gamma)$ of lossy lines consists of two components: the attenuation constant $(\alpha)$ and the phase constant $(\beta)$. In order to obtain the voltage wave equation in lossy transmission line, we need to modify the special case equation Eq. 3.25 into a general case equation by substituting $\gamma=\alpha+\mathbf{j} \beta$ in $\mathbf{j} \beta$.

$$
U(z=-l)=\frac{1}{2} I_{2}\left[Z_{2}\left(e^{(\alpha+\mathrm{j} \beta) l}+e^{-(\alpha+\mathrm{j} \beta) l}\right)+Z_{\mathrm{o}}\left(e^{(\alpha+\mathrm{j} \beta) l}-e^{-(\alpha+\mathrm{j} \beta) l}\right)\right] .
$$

By benefiting from the hyperbolic functions $\left(\sinh (x)=\frac{e^{x}-e^{-x}}{2}\right)$ and $\left(\cosh (x)=\frac{e^{x}+e^{-x}}{2}\right)$, 
Eq. 3.29 can be written as the following

$$
U(z=-l)=I_{2} Z_{2} \cosh (\alpha+\mathrm{j} \beta) l+I_{2} Z_{\mathrm{o}} \sinh (\alpha+\mathrm{j} \beta) l
$$

The following relationship between hyperbolic and trigonometric functions $(\sinh (x)=-\mathrm{j} \sin (\mathrm{j} x))$ and $(\cosh (x)=\cos (\mathrm{j} x))$ are used to simplify the expression

$$
\begin{aligned}
U(z=-l) & =I_{2} Z_{2} \cos \mathrm{j}(\alpha+\mathrm{j} \beta) l-\mathrm{j} I_{2} Z_{\mathrm{o}} \sin \mathrm{j}(\alpha+\mathrm{j} \beta) l \\
& =I_{2} Z_{2} \cos (\mathrm{j} \alpha-\beta) l-\mathrm{j} I_{2} Z_{\mathrm{o}} \sin (\mathrm{j} \alpha-\beta) l
\end{aligned}
$$

To decompose the sine and cosine arguments of equation above, the sum and difference formulas for trigonometric equations have been used

$$
\begin{aligned}
U(z=-l)= & I_{2} Z_{2}[\cos (\mathrm{j} \alpha l) \cos (\beta l)+\sin (\mathrm{j} \alpha l) \sin (\beta l)] \\
& -\mathrm{j} I_{2} Z_{\mathrm{o}}[\sin (\mathrm{j} \alpha l) \cos (\beta l)-\cos (\mathrm{j} \alpha l) \sin (\beta l)] .
\end{aligned}
$$

For transmission line with quarter-wave length $(l=\lambda / 4)$, the voltage equation becomes

$$
U\left(l=\frac{\lambda}{4}\right)=I_{2} Z_{2} \sin (\mathrm{j} \alpha l)+\mathrm{j} I_{2} Z_{\mathrm{o}} \cos (\mathrm{j} \alpha l) .
$$

By rearranging Eq. 3.33, the load current can be expressed as

$$
I_{2}=\frac{U\left(l=\frac{\lambda}{4}\right)}{Z_{2} \sin (\mathrm{j} \alpha l)+\mathrm{j} Z_{\mathrm{o}} \cos (\mathrm{j} \alpha l)}
$$

From Eq. 3.34, we conclude that the lossy quarter-wave length cable has no influence on the phase relationship between the load current and input voltage. 


\section{Half-wave transmission line}

In Fig. 3.12, the PA output is connected to the coil by a multiple of half-wave length $(N \cdot \lambda / 2)$ cable. In this subsection we try to investigate the influence of lossy transmission line on the relationship between the input and output voltages of multiple half-wave length cable. Starting from Eq. 3.32

$$
U\left(l=N \cdot \frac{\lambda}{2}\right)=-I_{2} Z_{2} \cos (\mathrm{j} \alpha l)+\mathrm{j} I_{2} Z_{\mathrm{o}} \sin (\mathrm{j} \alpha l) .
$$

By rearranging Eq. 3.35, and substituting $\frac{U_{2}}{Z_{2}}$ in $I_{2}$, the load voltage in terms of hyperbolic function becomes

$$
U_{2}=\frac{-Z_{2} U\left(l=N \cdot \frac{\lambda}{2}\right)}{Z_{\mathrm{o}} \sinh (\alpha l)+Z_{2} \cosh (\alpha l)} .
$$

For lossless transmission line the load voltage becomes

$$
U_{2}= \pm U\left(l=N \cdot \frac{\lambda}{2}\right)
$$

In our new concept as illustrated in Fig. 3.12, a multiple of half-wave length cable is employed to connect the power amplifier to the coil. Eq. 3.37 shows that the load voltage at the end of a half-wave lossless transmission line is independent of load impedance and cable characteristic impedance. Rather it lags the input voltage by $180^{\circ}$. Whereas Eq. 3.36 shows the dependence on those two parameters in case of lossy cable. By using a lossy multiple half-wave length cable, the unity voltage ratio of the output to the input $\left(U_{2} / U(l)\right.$ ) ( see Fig 3.13) starts to decrease as a function of length as seen in Fig. 3.14 (a), by using $R G 223$ cable with characteristic impedance $Z_{o}=50 \Omega$, inner conductor diameter $=0.9 \mathrm{~mm}$, dielectric $(\mathrm{PE})$ diameter $=2.95 \mathrm{~mm}$, and attenuation factor of $0.23 \mathrm{~dB} / \mathrm{m}$ at $298 \mathrm{MHz}$. In addition, the phase ratio deviates from the $180^{\circ}$ level as seen in Fig. 3.14 (b). In our application of Fig. 3.12, $U(l)$ is the probe-voltage $U_{\mathrm{I}}$ which is used to control the current $I_{2}$. The discrepancy between $U(l)$ and $U_{2}$ in Eq. 3.36 leads to an error for the control voltage $U_{\mathrm{I}}$, such that the current $I_{2}$ is over-estimated with a phase error 


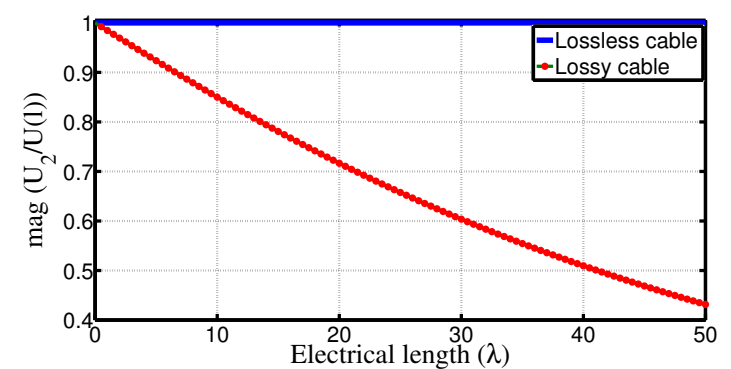

(a)

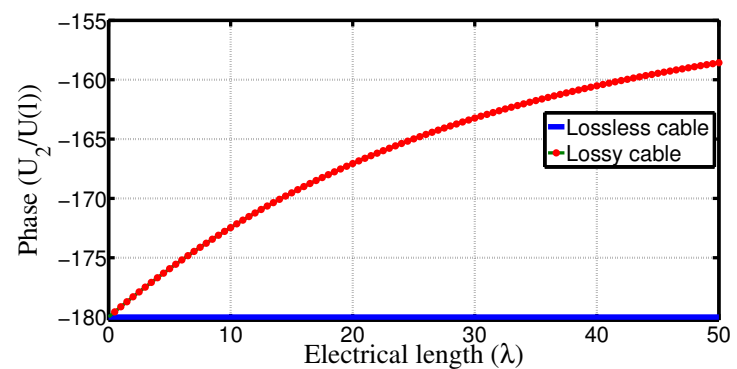

(b)

Figure 3.14: The influence of attenuation $(\alpha=0.23 \mathrm{~dB} / \mathrm{m})$ in (multiple) half-wave cable on the ratio of the load voltage to the input voltage with load impedance $Z_{2}=25+\mathrm{j} \cdot 25 \Omega$. (a) Magnitude, (b) phase.

depending on the actual coil impedance. The accuracy problem of measurement at the other end of the cable can also be seen in Smith chart by measuring the reflection coefficient comparing lossless and lossy half-wave cable. Starting from the reflection coefficient definition at any point on the cable

$$
\Gamma_{l}=\frac{U_{\mathrm{o}}^{-} e^{-\gamma l}}{U_{\mathrm{o}}^{+} e^{\gamma l}} .
$$

The reflection coefficient at the load $(l=0)$ is

$$
\Gamma_{2}=\frac{U_{\mathrm{o}}^{-}}{U_{\mathrm{o}}^{+}}=\frac{Z_{2}-Z_{\mathrm{o}}}{Z_{2}+Z_{\mathrm{o}}}
$$

and for $l=N \cdot \lambda / 2$, Eq. 3.38 becomes

$$
\Gamma_{N \cdot \frac{\lambda}{2}}=\Gamma_{2} e^{-2 \gamma N \cdot \frac{\lambda}{2}}=\Gamma_{2} e^{-2 \alpha N \cdot \frac{\lambda}{2}}
$$

For lossless cable, $\Gamma_{N \cdot \frac{\lambda}{2}}=\Gamma_{2}$, whereas the reflection coefficient by using lossy cable converges to zero for very long cable as described in Eq. 3.40. Fig. 3.15 shows the reflection coefficient calculated at the other end of a lossless and a lossy (multiple) half-wave cable when a load impedance $Z_{2}=25+\mathrm{j} \cdot 25 \Omega$ is connected. 


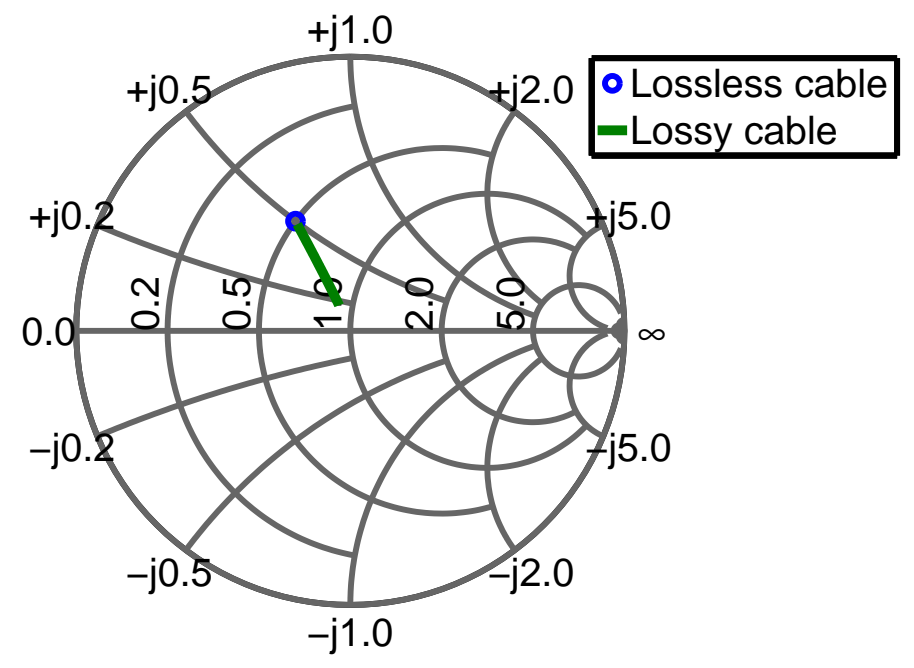

Figure 3.15: Reflection coefficient calculated after lossless and lossy (multiple) half-wave cable in the range between $(\lambda / 2)$ and $100 *(\lambda / 2)$ and load impedance $Z_{2}=25+\mathrm{j} \cdot 25 \Omega$.

\subsubsection{Design of a $1 \mathrm{~kW}$ power amplifier}

The near-magnet power amplifier reported in [53] was designed and produced at the High Frequency Technology department, Duisburg-Essen University. The initial design of the highpower stage was based on the manufacturer's data sheet, in particular the output matching circuit topology was adopted and later modified based on circuit simulation and experiment. In the following, the design and simulation results are presented while experimental results can be found in the appendix A and B. The near-magnet power amplifier has been designed to work at the Larmor frequency for $7 \mathrm{~T}(298 \mathrm{MHz})$ and to deliver up to $1 \mathrm{~kW}$ RF power to a matched load. It consists of two amplification stages: the MRF6V2010 in a driver stage and the BLF188XR LDMOS field effect push-pull transistor pair in a balanced final stage as shown in Fig. 3.16. To design our power amplifier, we need matching networks for both transistors in order to deliver maximum output power. The conjugate matching method based on small signal S-parameter analysis is generally used in small-signal amplifier design to achieve the maximum gain. However, our power amplifier deals with large signals and its output impedance is power dependent. Therefore, a matching method based on large-signal analysis is required. The EM/Circuit Co-Simulation feature in Agilent ADS software which enables to combine results from EM simulation with large-signal circuit simulation, was used for the design. 


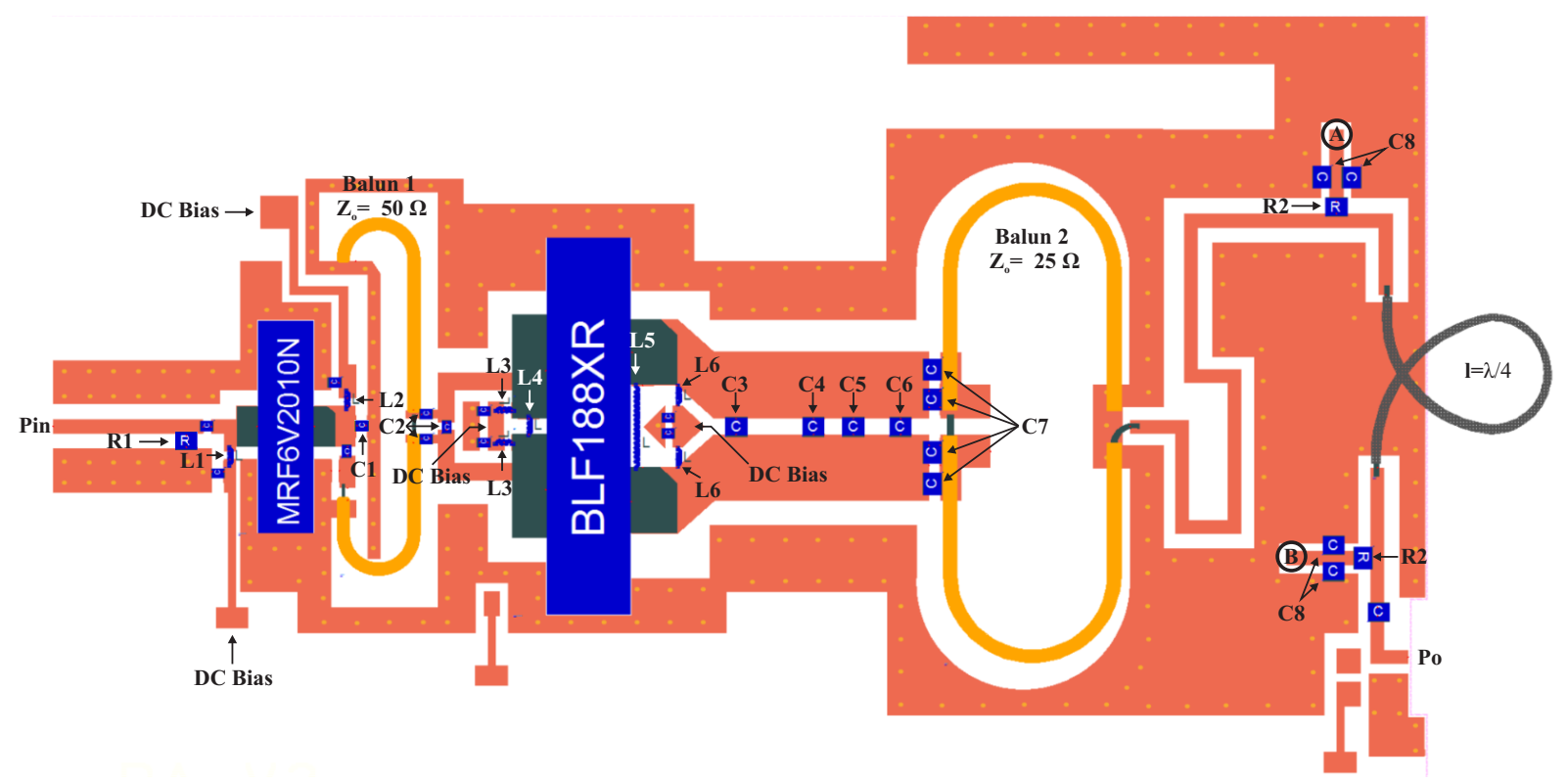

Figure 3.16: Simulation setup in ADS software for 2-stage $1 \mathrm{~kW}$ power amplifier for 7T MRI.

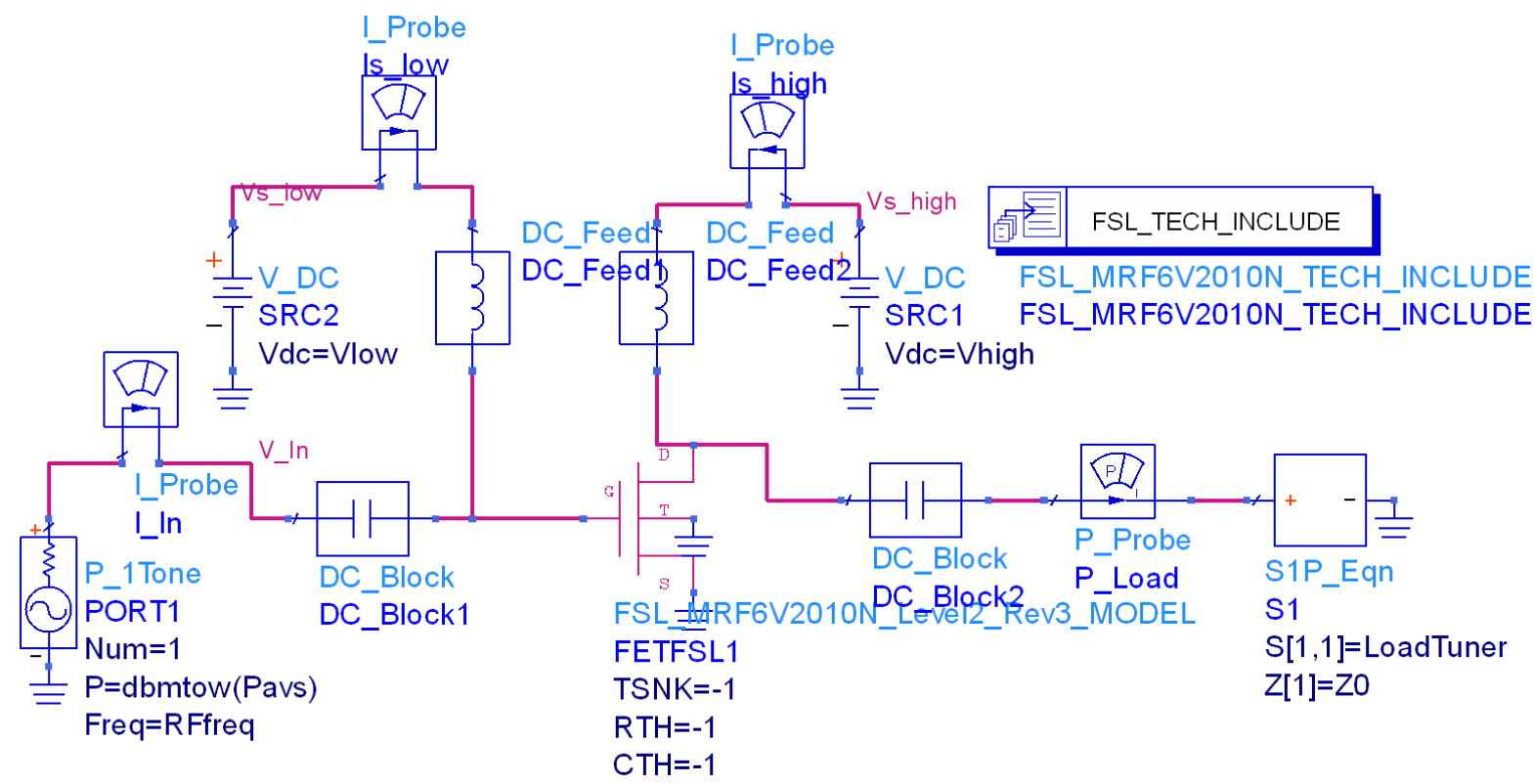

Figure 3.17: Load pull simulation setup for the driver stage transistor (MRF6V2010).

\section{Load Pull Analysis}

The load pull technique $[55,56]$ is the most common method for power matching of power amplifiers. It is considered fast, easy and accurate method and is implemented in different CAD tools. In Agilent ADS software, a load pull simulation setup is available and ready to use after replacing the existing transistor with ours. In simulation, the load pull technique is performed based on the harmonic balance simulation. Harmonic balance is a powerful technique for the analysis of largely nonlinear devices. It is a frequency domain analysis technique for simulating 


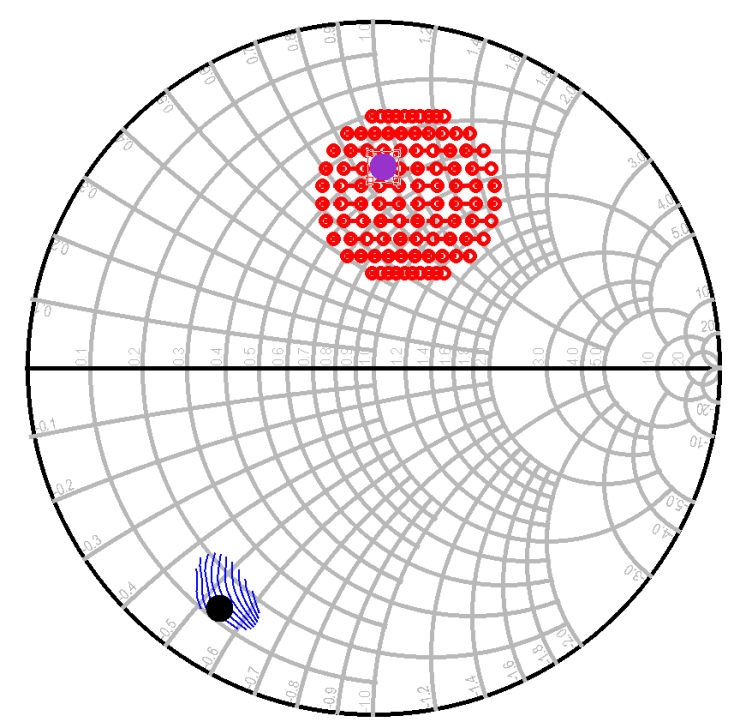

(a)

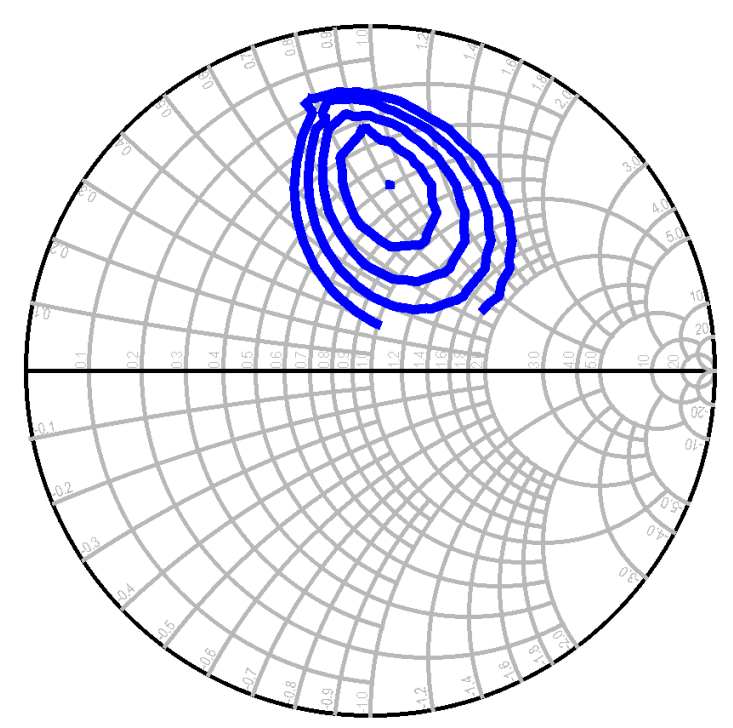

(b)

Figure 3.18: Load pull simulation results for the MRF6V2010 transistor. (a) Region of load impedances covered by load tuner, (b) contours of equal power delivered with step size $0.5 \mathrm{~dB}$.

distortion arising form these devices. It has the ability to calculate the steady-state spectral content of voltages or currents. Fig. 3.17 shows the impedance load pull simulation setup for the driver stage transistor (MRF6V2010) as it appears in ADS workspace. It employs a load tuner to vary the load impedance presented to the transistor to monitor a set of performance parameters such as output power, DC power consumption, gain, power added efficiency and other parameters. To perform such a simulation, several settings should be specified. For the MRF6V2010 transistor, we have specified the following settings: input frequency is $298 \mathrm{MHz}$, input drive power is $14 \mathrm{dBm}$, input $\mathrm{DC}$ bias is $3.3 \mathrm{~V}$ to drive the transistor in class $\mathrm{AB}$, output DC bias is $50 \mathrm{~V}$, the order of generated harmonics is 5 . In addition, we need to specify a circular region for load reflection coefficients on the Smith chart. This region appears in Fig. 3.18 (a) as circular red area where the blue area on the same Smith chart represents the corresponding input impedances which we have to provide conjugate matching. Inside the red area there is a small violet circle which represents the optimum load impedance for maximum power transfer. The corresponding input impedance is indicated by a small black circle. For the MRF6V2010 transistor, the optimum load impedance that has been obtained is $25.88+\mathrm{j} \cdot 44.60 \Omega$ while the corresponding input impedance is $8.31-\mathrm{j} \cdot 22.83 \Omega$. The maximum output power which can be delivered with this combination of impedances is $40.5 \mathrm{dBm}$. According to the specified reflection coefficient region and the total number of generated reflection coefficient points (the desired total number of points is specified before running the simulation), the load pull simulation will 


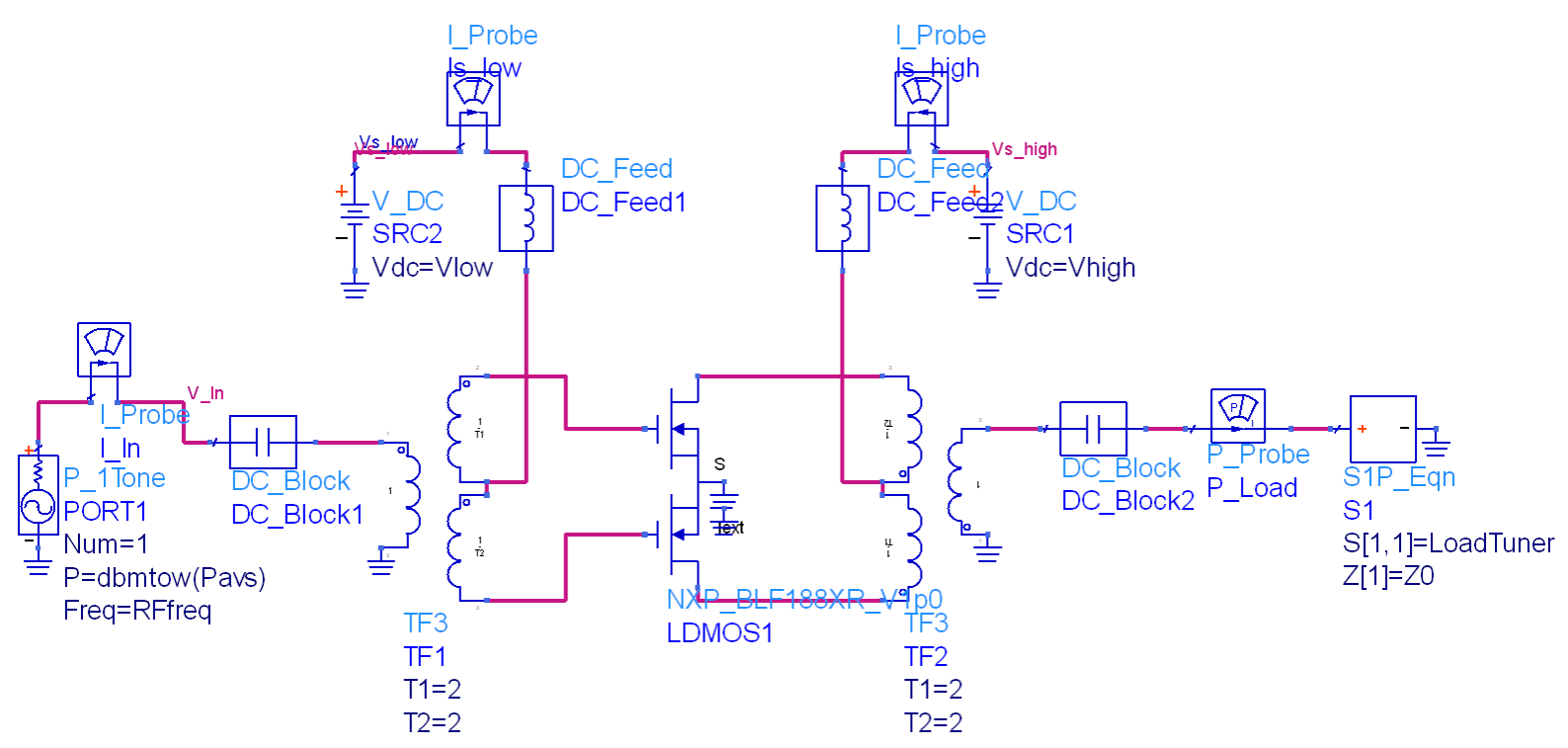

Figure 3.19: Load pull simulation setup for the final stage transistor (BLF188XR).

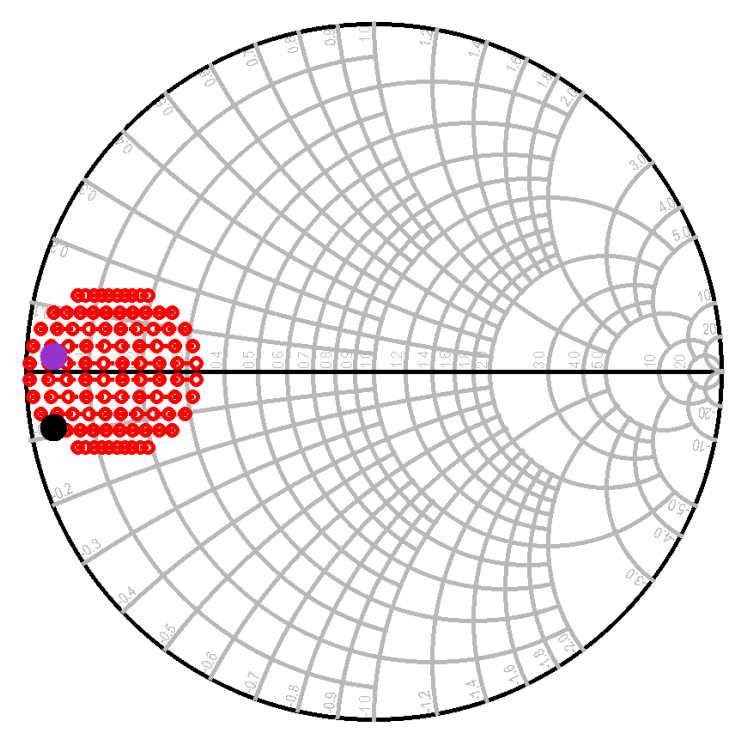

(a)

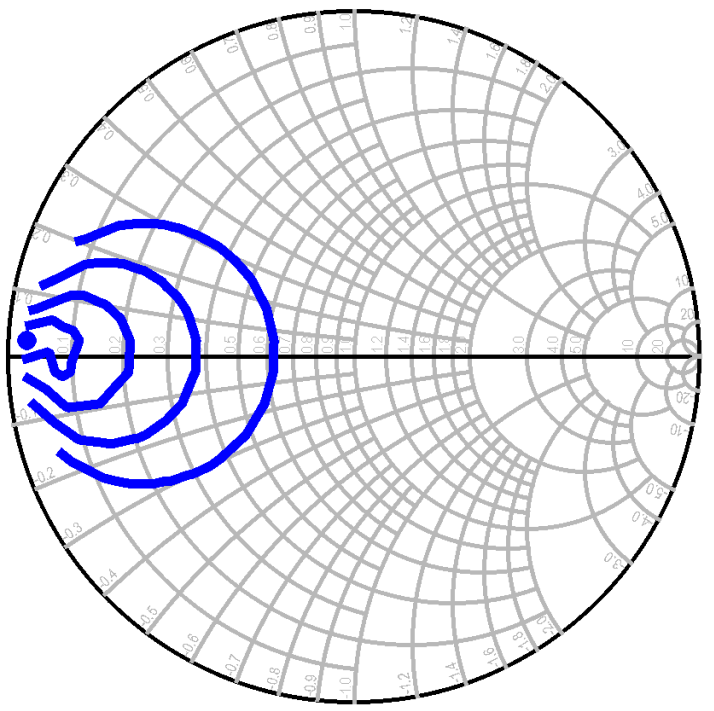

(b)

Figure 3.20: Load pull simulation results for BLF188XR transistor. (a) Region of load impedances covered by load tuner, (b) contours of equal power delivered with step size $2 \mathrm{~dB}$.

create a set of isocontours on a Smith chart where each contour includes impedance values that can provide the same output power. These contours converge to a single point as seen in Fig. 3.18 (b). The single point represents the optimum load impedance with maximum output power of $40.5 \mathrm{dBm}$, while the other contours are distributed away from the maximum power by a step size of $0.5 \mathrm{~dB}$. This matching method has been also applied to the final stage transistor BLF188XR where the load pull simulation setup is shown in Fig. 3.19. A center-tapped transformer is used with a turns ratio of 1 to analyze the actual output impedances. Therefore, 
the turns ratio between the primary and a half of the secondary (T1 and T2) is 2. To perform the load pull simulation for the BLF188XR transistor, we have specified the following settings: input frequency is $298 \mathrm{MHz}$, input power is $37 \mathrm{dBm}$, input $\mathrm{DC}$ bias is $2 \mathrm{~V}$ to drive the transistor in class $\mathrm{AB}$, output $\mathrm{DC}$ bias is $50 \mathrm{~V}$, the order of generated harmonics is 5 , and the specified region for load reflection coefficients on the Smith chart is shown in Fig. 3.20 (a). The optimum load impedance that has been obtained for maximum power transfer is $1.95+\mathrm{j} \cdot 1.8 \Omega$ while the corresponding input impedance is $1.64-\mathrm{j} \cdot 4.31 \Omega$. The maximum output power which can be delivered with this combination of impedances is $61.54 \mathrm{dBm}$. The power contours that have been created on Smith chart with step size $2 \mathrm{~dB}$ are shown in Fig. 3.20 (b).

\section{Matching Network}

After obtaining the large signal input and output load impedances of both transistors, we are now able to build the matching network for our PA. Firstly, we have to match the large signal input impedance of the MRF6V2010 transistor to the $50 \Omega$ generator impedance. A single shunt inductor of $12 \mathrm{nH}$ indicated by $L 1$ with a single shunt resistor of $140 \Omega$ indicated by $R 1$ in Fig. 3.16 can satisfy this purpose. For the interstage matching network between the transistors, a symmetrical coaxial balun (Balun 1) has been used to transform the single-ended microstrip line (the output of driver transistor) into differential microstrip line (the input of push-pull transistor). This balun contributes to the impedance transformation with the interstage matching network. The design of the symmetrical coaxial cable balun with the corresponding equivalent circuit is illustrated in Fig. 3.21. The equivalent circuit consists of a transmission line (TL) which has the same characteristics as the coaxial balun (characteristic impedance $\left(Z_{\mathrm{o}}\right)$, permittivity

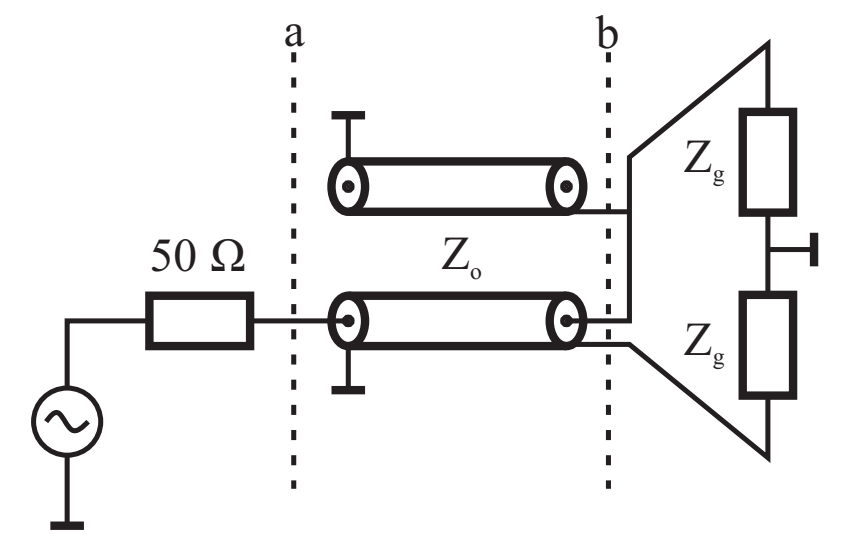

(a)

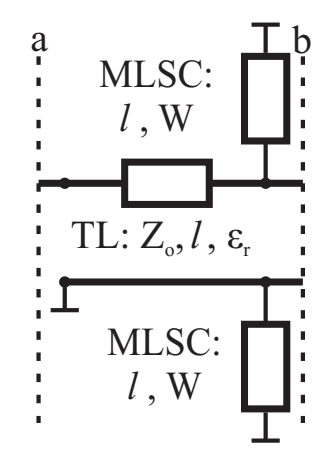

(b)

Figure 3.21: (a) Symmetrical coaxial balun, (b) symmetrical balun equivalent circuit. 


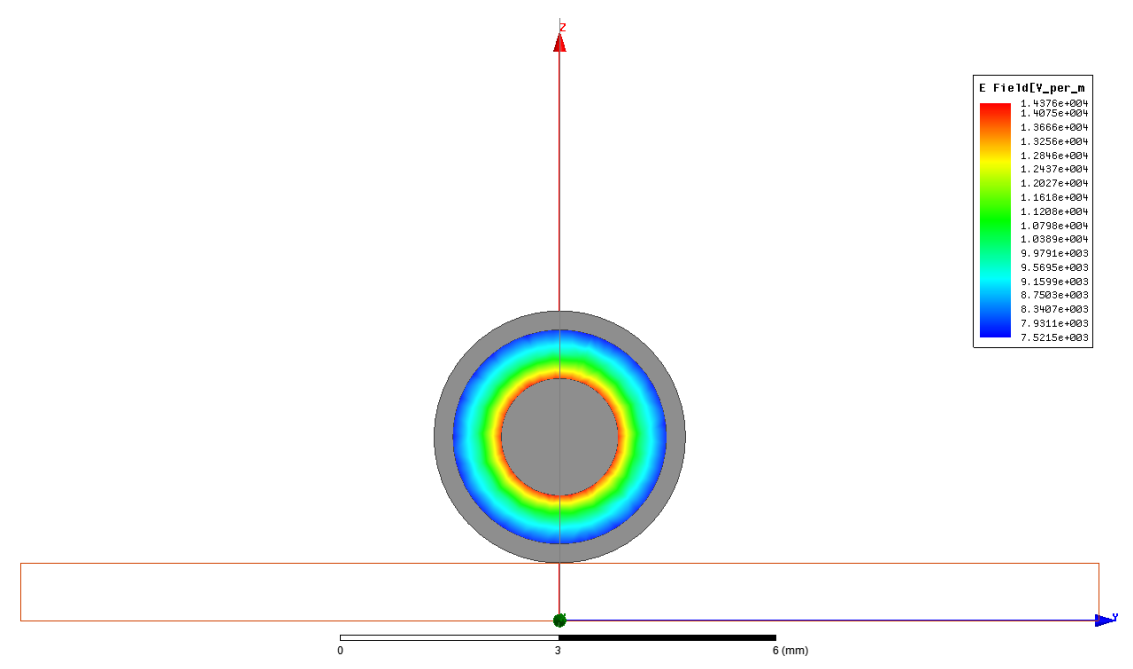

(a)

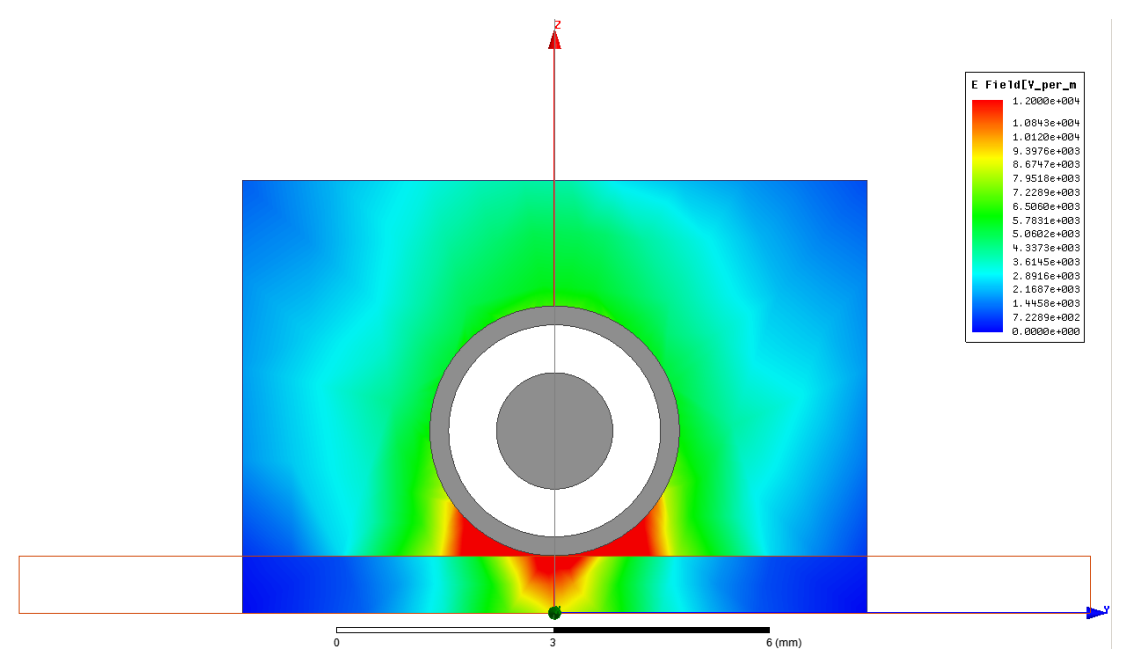

(b)

Figure 3.22: E-field pattern for balun coaxial cable. (a) First mode, (b) second mode.

$\left(\varepsilon_{\mathrm{r}}\right)$, the length $(l)$ ), and two microstrip short-circuited stubs (MLSC), representing the leakage inductance of the coaxial balun. The leakage inductance has been calculated using an FEM tool (Ansoft HFSS). For the balun that is used in the interstage matching network, the simulation setup assumes a hand formable coaxial cable (SUCOFORM 86) with $30 \mathrm{~mm}$ length, $Z_{\mathrm{o}}=50 \Omega$, inner conductor diameter $=0.53 \mathrm{~mm}$, dielectric $($ PTFE$)$ diameter $=1.63 \mathrm{~mm}$, outer conductor diameter $=2.1 \mathrm{~mm}$, on Rogers 4003 material $\left(\varepsilon_{\mathrm{r}}=3.55, \mathrm{~h}=0.81 \mathrm{~mm}\right)$. A wave port has been defined at the coaxial cable cross section in order to excite the cable with different modes. The first mode has been defined between the inner and outer conductors to analyse the characteristic impedance of the cable, whereas the second mode has been defined to analyse the characteristic impedance of the mode existing between the outer conductor and the ground plane. Fig. 3.22 shows the electric field patterns for both modes as appearing in HFSS simulation software. The calculated 
impedance from the first mode is basically the characteristic impedance of the cable that is provided by the manufacturer which is about $50 \Omega$. The calculated characteristic impedance of the second mode is about $Z_{\mathrm{m}}=38 \Omega$. By having the physical length of the cable $(l=30 \mathrm{~mm})$, the electrical length $(E=\beta l)$ at the operating frequency $(298 \mathrm{MHz})$ equals $15.557^{\circ}$. From the mode impedance $\left(Z_{\mathrm{m}}\right)$ and electrical length $(E)$, we can calculate the leakage inductance by $\mathrm{j} \omega L=$ $\mathrm{j} Z_{\mathrm{m}} \tan (\beta l)$ which is about $4.5 \mathrm{nH}$. In order to represent the balun leakage inductance in its correct frequency dependence it was modelled as a MLSC with width $(W)=2.7 \mathrm{~mm}$ and length $(l)=$ $25.27 \mathrm{~mm}$. The MLSC parameters have been calculated using Advanced Design System software (ADS). The lumped elements which have been used to build the interstage matching network are indicated in Fig. 3.16 and have the following values: $L 2=27 \mathrm{nH}, L 3=43 \mathrm{nH}, L 4=6.8 \mathrm{nH}$, $C 1=5.6 \mathrm{pF}, C 2=47 \mathrm{pF}$.

For the output matching network, another balun was used with opposite functionality in comparison to that used in the interstage matching network. A semi-rigid cable (DA25141) with $55 \mathrm{~mm}$ length, $Z_{o}=25 \Omega$, inner conductor diameter $=1.63 \mathrm{~mm}$, dielectric $(\mathrm{PTFE})$ diameter $=2.97 \mathrm{~mm}$, and outer conductor diameter $=3.58 \mathrm{~mm}$, has been used as a last stage in the matching network. Similar to the first balun, we have simulated the second balun to analyse the characteristic impedances for the interesting modes. From the first mode, the calculated impedance shows the characteristic impedance of the cable which is about $25 \Omega$, whereas the characteristic impedance calculated for the second mode is about $Z_{\mathrm{m}}=32 \Omega$. By following a similar procedure of the Balun 1 to derive the corresponding equivalent circuit for the second balun, we obtain an $9.37 \mathrm{nH}$ inductance value for the grounded inductors. The equivalent MLSC has a width $(W)=3.43 \mathrm{~mm}$ and length $(l)=46.47 \mathrm{~mm}$. The output matching network employs a multistage matching network to increase the bandwidth of matching for the balanced transistor. The lumped elements for this network are indicated in Fig. 3.16 and have the following values: $L 5=5.6 \mathrm{nH}, L 6=5.1 \mathrm{nH}$, $C 3=10 \mathrm{pF}, C 4=27 \mathrm{pF}, C 5=20 \mathrm{pF}, C 6=10 \mathrm{pF}, C 7=180 \mathrm{pF}$.

\section{The Voltage Probes}

As mentioned before and shown in Fig. 3.12, the near-magnet power amplifier utilizes two probes across a quarter-wave transmission line. The first probe voltage $\left(U_{\mathrm{U}}\right)$ is proportional to the voltage at the coil terminals $\left(U_{2}\right)$, whereas the second probe voltage $\left(U_{\mathrm{I}}\right)$ is proportional to the current into the coil $\left(I_{2}\right)$. Position $A$ after the second balun in Fig. 3.16 indicates the probe for $U_{\mathrm{U}}$, whereas position $B$ indicates the probe for $U_{\mathrm{I}}$. Both probes use a $22 \mathrm{k} \Omega$ resistor $(R 2)$ in combination with a $40 \mathrm{pF}$ shunt capacitor $(C 8)$ and a $50 \Omega$ termination connected to a coaxial 
cable (not shown) to probe the microstrip line, thus creating a broad-band voltage divider of about $54 \mathrm{~dB}$ probe attenuation.

\subsubsection{Characterization of the $1 \mathrm{~kW}$ power amplifier}

In general, the quality of power amplifiers are characterized by a list of specifications such as the output power, the gain, the phase shift and the efficiency, see section 3.5. By biasing the driver transistor and the high-power transistor of the power amplifier with appropriate operating points, the characteristics of the power amplifier can be manipulated. Fig. 3.23 shows the basic characteristics of our PA by driving the two transistors with different operating points. These results have been simulated by applying an unmodulated sinusoidal signal at $298 \mathrm{MHz}$ operating frequency to the input port whereas the output port is matched terminated. The optimal combination of operating points in order to obtain the best linearity for the PA was when the driver transistor was biased by $3.3 \mathrm{~V}$ (Dr) and the power transistor was biased by $2 \mathrm{~V}$ (PA). The maximum output power (saturated power) that can be delivered by the PA to a matched load is around $62 \mathrm{dBm}$. At optimal operating points, the PA shows a gain of $52.5 \mathrm{~dB}$ in the linear region, whereas the error in phase shift between driving in the linear region and the saturated region is around $30^{\circ}$. The linear amplification region $\left(\mathrm{P}_{1 \mathrm{~dB}}\right)$ ends at about $800 \mathrm{~W}$. The power added efficiency (PAE) at saturation can reach $80 \%$ maximum. The PA characteristic in terms of frequency response can be investigated by plotting the PA gain as a function of frequency as seen in Fig. 3.23(e) which shows a wide bandwidth for a drive level in the linear region.

Fig. 3.24 demonstrates how the optimal load impedance is output power dependent. To deliver a maximum power from the PA, the output port should be terminated by $50 \Omega$ impedance, whereas the optimal load impedance shifts to lower impedance value when the PA is driven to lower output power as illustrated in Fig. 3.24(b).

\section{Harmonics and Intermodulation Distortion}

The linearity of power amplifiers are mainly determined by two common measurements: The $1-\mathrm{dB}$ compression $\left(\mathrm{P}_{1 \mathrm{~dB}}\right)$ point and the output intercept of $3 \mathrm{rd}$ order OIP3 point. The $\mathrm{P}_{1 \mathrm{~dB}}$ is defined by the input power at which the output power decreases $1 \mathrm{~dB}$ from its normal linear response. Once the power amplifier is driven into compression, it starts to behave nonlinearly and produces harmonics. Fig. 3.25 shows the nonlinear products caused by the second and third order nonlinearity as a function of input power when the PA is tested using a single tone. If the 


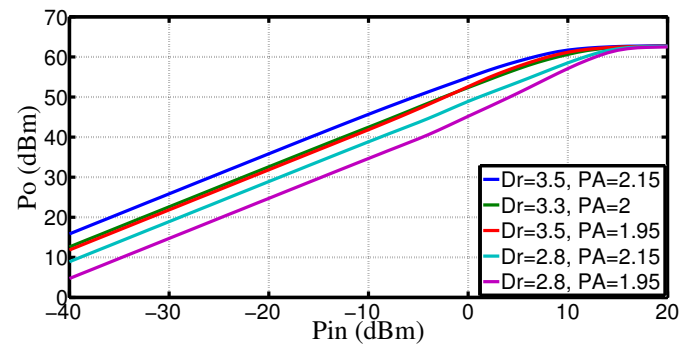

(a)

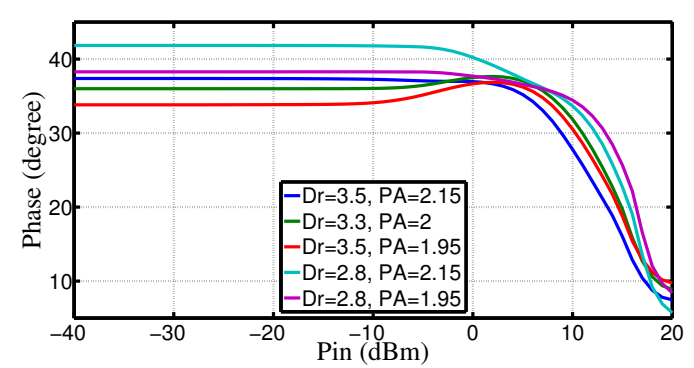

(c)

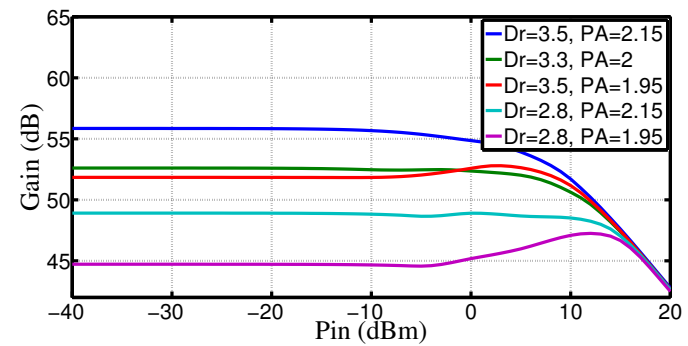

(b)

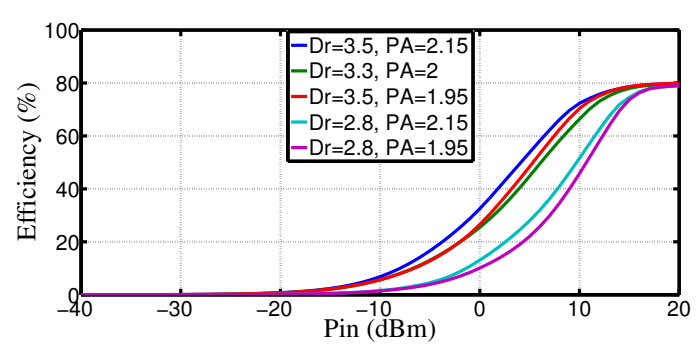

(d)

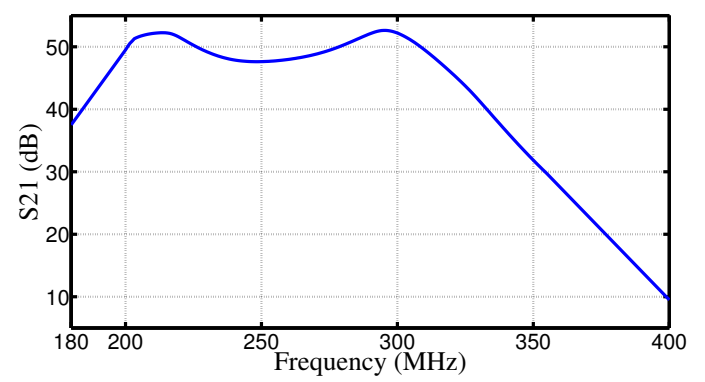

(e)

Figure 3.23: The characteristics of the PA by different operating points. (a) Output power, (b) gain, (c) phase shift, (d) efficiency, (e) gain vs frequency at bias voltages $\mathrm{Dr}=3.3 \mathrm{~V}$ and $\mathrm{PA}=2 \mathrm{~V}$.

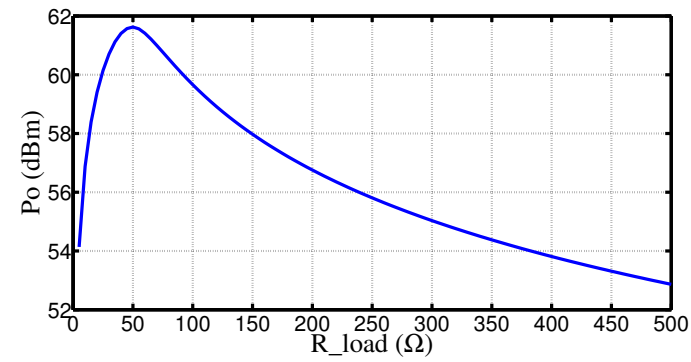

(a)

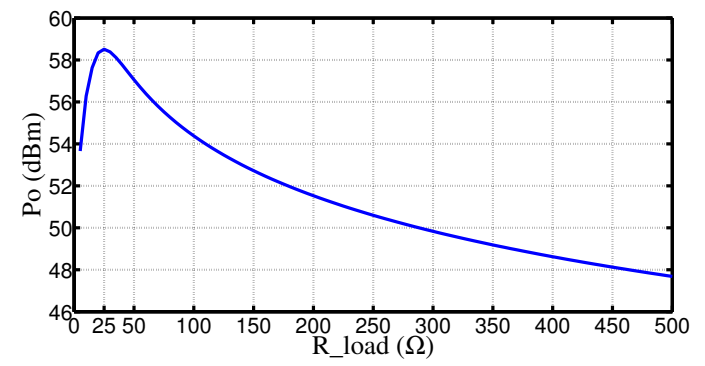

(b)

Figure 3.24: Output power vs. load impedance. (a) At maximum drive power, (b) when driven for output power of $500 \mathrm{~W}$ with $50 \Omega$ load impedance. 
PA is driven into compression and tested using two tones at $f_{1}$ and $f_{2}$ close together in frequency, intermodulation distortion is produced which contains the third-order products at $2 \mathrm{f}_{1}-\mathrm{f}_{2}$ and $2 \mathrm{f}_{2}-\mathrm{f}_{1}$ near the input signals. Fig. 3.26 shows the OIP3 point due to two test signals.

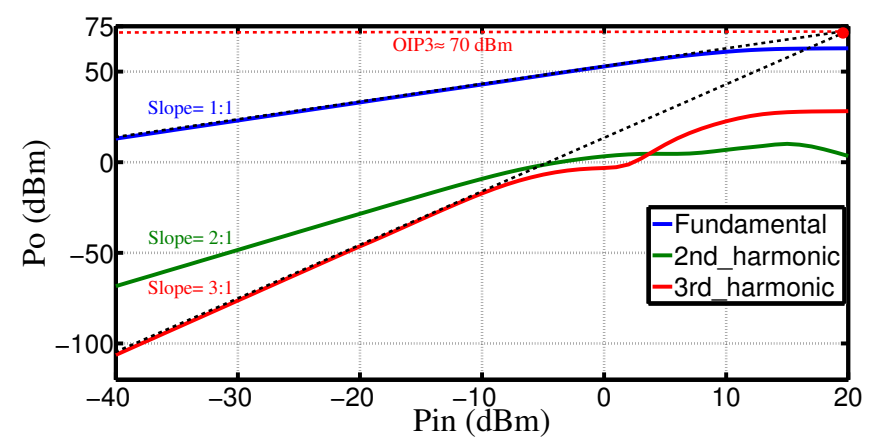

Figure 3.25: Transfer characteristic for fundamental, 2nd-order and 3rd-order signals of a single test signal.

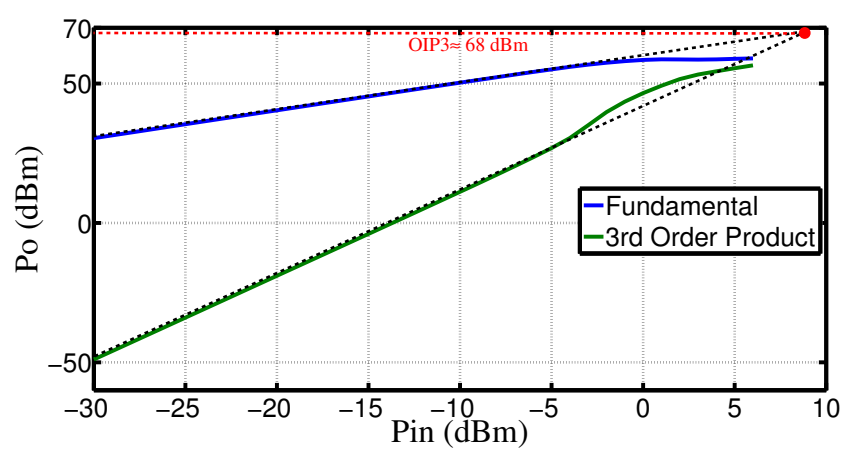

Figure 3.26: Transfer characteristic for fundamental and 3rd-order signals of a two test signals.

\subsubsection{Stability Analysis}

Instabilities in power amplifiers are considered the major concern for the designers. It is often caused by an internal feedback which leads a part of the output signal to couple into the input and makes the power amplifier to oscillate once the positive feedback conditions are fulfilled at some frequency. Oscillations in power amplifiers are well-known as parametric oscillations which are associated with the drive power of the amplifier, the operating frequency, the bias level, and the load impedance. For small-signal power amplifiers, the stability can be determined by its S-parameters using the Rollett's stability factor $K$ [57]. In case of large-signal power amplifier, a nonlinear analysis technique maybe required to solve complex mathematical derivations. A widely used technique to analyze oscillations in PAs based on the nonlinear steady state Harmonic Balance (HB) method has been proposed in [58,59]. It makes use of a small-signal injection 
current source $i_{\text {in }}$ at frequency $f_{\mathrm{s}}$ connected to a circuit node $n$ as shown in Fig 3.27, to obtain a closed-loop transfer function of the circuit [60]. The frequency response is calculated as the impedance seen at this node by sweeping the frequency $f_{\mathrm{s}}$ :

$$
H(\mathrm{j} \omega)=Z(\mathrm{j} \omega)=\frac{v_{\text {out }}(\mathrm{j} \omega)}{i_{\text {in }}(\mathrm{j} \omega)}
$$

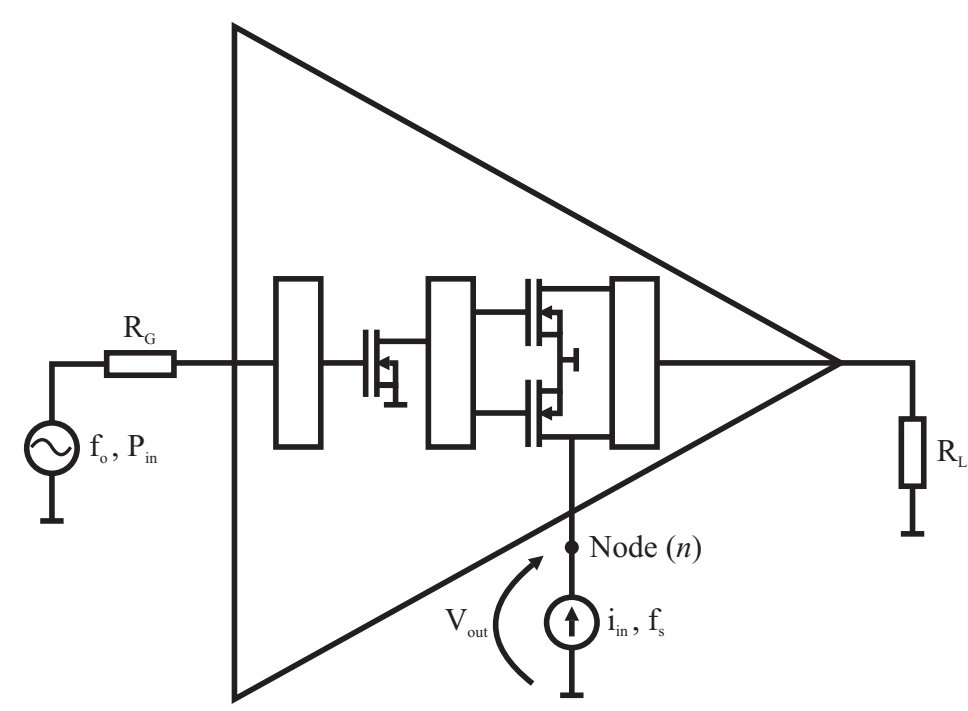

Figure 3.27: The Stability analysis technique utilizes a sinusoidal small-signal current source $i_{\text {in }}$ with frequency $f_{\mathrm{s}}$.

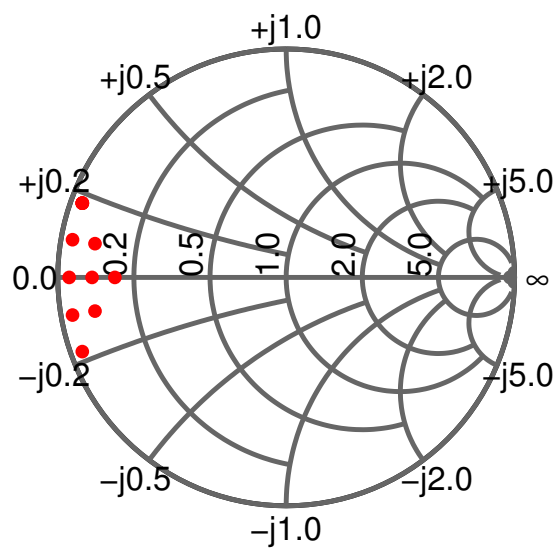

Figure 3.28: Unstable region in the reflection coefficient plane for output power of $60 \mathrm{dBm}$ at $298 \mathrm{MHz}$.

The large-signal stability analysis utilizes the conversion matrix analysis, or large-signal/small- 


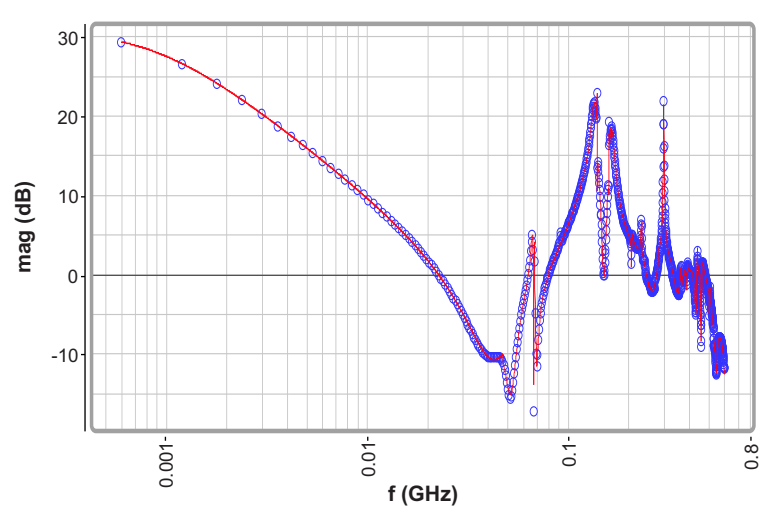

(a)

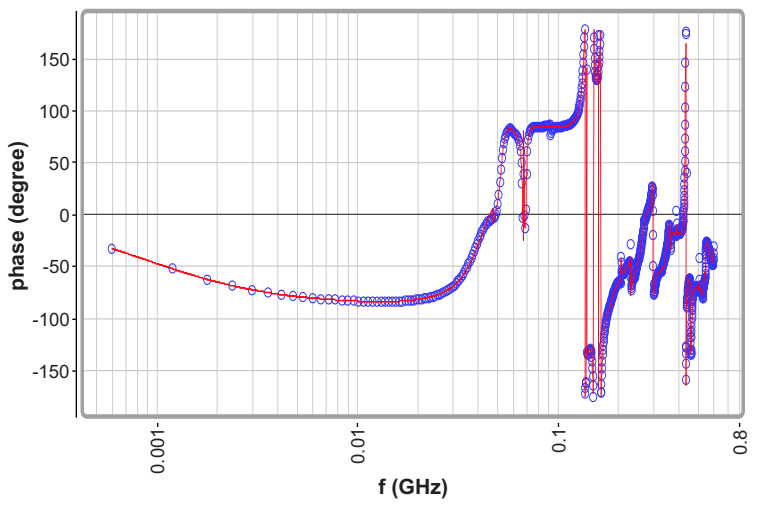

(b)

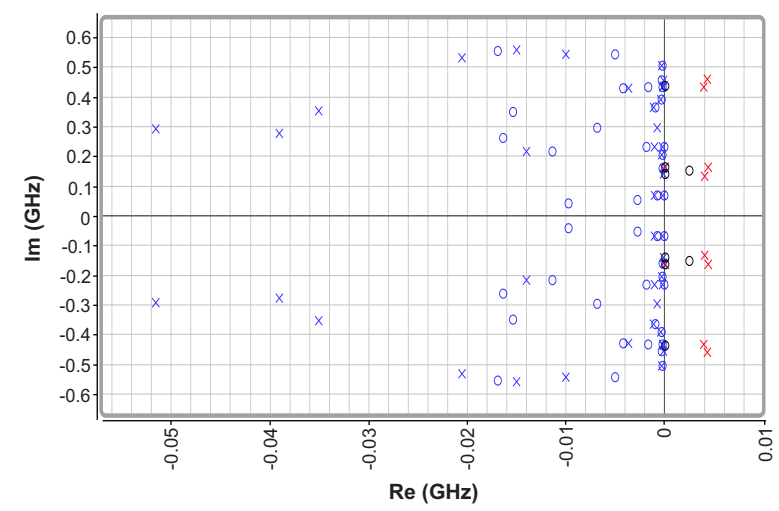

(c)

Figure 3.29: Simulated (circles) and identified (solid line) frequency response $H(j \omega)$ for $\Gamma_{\mathrm{L}}=$ $0.9 \angle 180^{\circ}$. (a) Magnitude, (b) phase, (c) associated pole-zero map ( $\times$ :poles, $\bigcirc$ :zeros).

signal analysis [61], to linearise the system about the steady-state periodic solution to calculate the small-signal response of the system under large-signal excitation. Investigation of the stability of a power amplifier may need to insert the small-signal current source at different nodes in order to detect the complete set of potential oscillation frequencies. Once the current source $i_{\text {in }}$ is defined and the node-voltage $v_{\text {out }}$ is determined, the impedance function $Z$ and the admittance function $Y$ can be calculated. These two functions are used in different identification techniques to detect potential oscillation of the system such as pole-zero identification technique, Kurokawa's condition and admittance plot [62]. The stability analysis for our PA has been performed over a wide range of load conditions (including loads with high reflection coefficient) and input power. Fig. 3.28 shows the critical area on the Smith chart that leads the PA to oscillate. The stability analysis has been accomplished by calculating the poles and the zeros of the impedance function $Z$ which represents the frequency response of an internal closed loop (not necessarily same as 


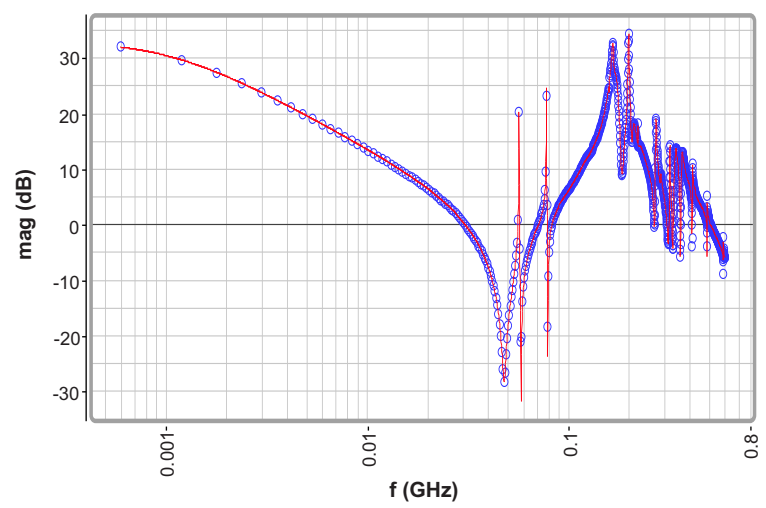

(a)

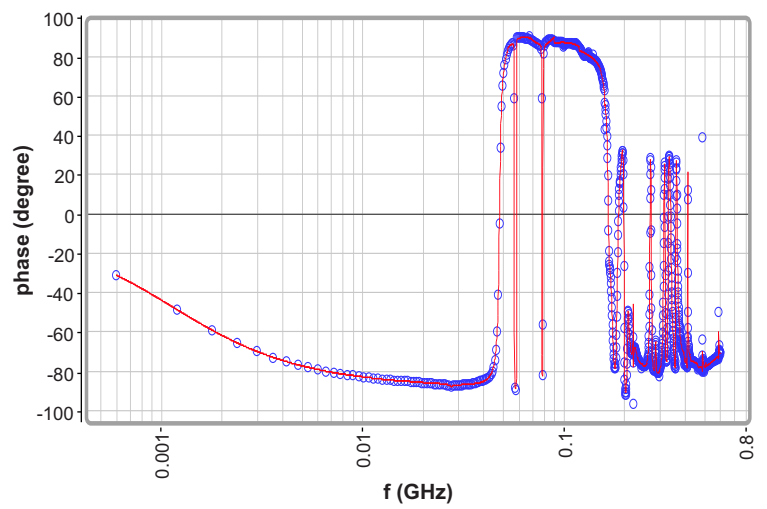

(b)

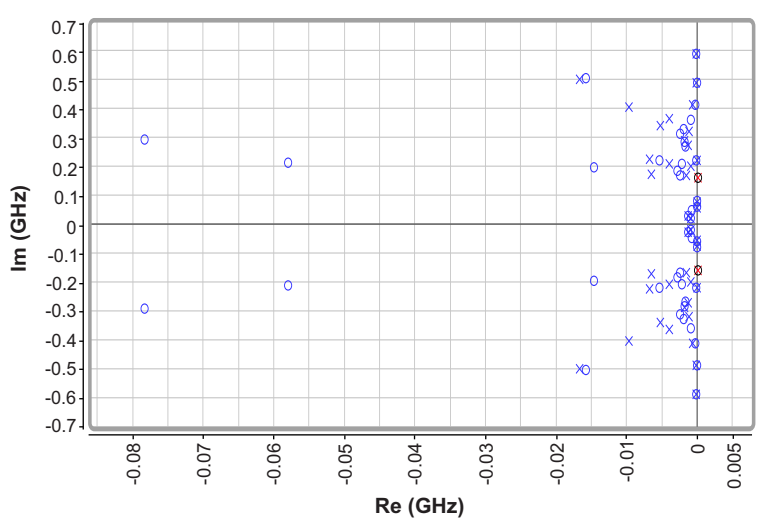

(c)

Figure 3.30: Simulated (circles) and identified (solid line) frequency response $H(\mathrm{j} \omega)$ for $\Gamma_{\mathrm{L}}=$ $0.9 \angle 180^{\circ}$ with bandstop filters. (a) Magnitude, (b) phase, (c) associated pole-zero map ( $\times$ :poles, $\bigcirc:$ zeros).

the over-all transfer function). Circuit stability requires that no poles with positive real parts exist on the complex plane. Fig. 3.29 shows the frequency response $H(j \omega)$ when the PA is terminated by a load with reflection coefficient $\Gamma_{\mathrm{L}}=0.9 \angle 180^{\circ}$, in addition to the associated poles and zeros plot. This system is unstable because some complex conjugate poles with positive real parts appear in the right-hand side of the complex plane. The location of the poles on the imaginary axis is equal to the oscillation frequency. For example in Fig. 3.29, the oscillation frequencies are $135 \mathrm{MHz}, 164 \mathrm{MHz}, 433 \mathrm{MHz}$ and $461 \mathrm{MHz}$. These results have been obtained using the stability analysis (STAN) software developed by AMCAD Engineering in combination with Advanced Design System (ADS) software. Different stabilization techniques have been considered in [63] to stabilize an L-band push-pull amplifier such as stabilization with an odd-mode stabilization resistor, stabilization with neutralization capacitors and stabilization with feedback resistors. In 
our case, we found that the easiest way to stabilize the power amplifier is to utilize two band-stop filter where the first filter rejects signals in the range between $130 \mathrm{MHz}$ and $170 \mathrm{MHz}$ while the second filter rejects signals in the range between $430 \mathrm{MHz}$ and $470 \mathrm{MHz}$. This way has proved its capability in stabilizing the PA for different load conditions and wide range of input power. Fig. 3.30 shows the frequency response $H(\mathrm{j} \omega)$ with the associated poles and zeros ploted after stabilizing the power amplifier for the same load mentioned before that leads to instability. Our experimental PA prototype [53] is always stable over the entire Smith chart area and under varying the input power without using the band-stop filters. This mismatch in stability results between simulation and measured PA maybe due to a variety of causes: First, the intermediate matching network was modified to reduce the gain around $230 \mathrm{MHz}$. In addition, the lumped elements used in the matching network are ideal components while the real components in the prototype are associated with parasitics. Another cause might be an inaccurate model of the bias networks in simulation, since resistive networks were used in the prototype.

\subsubsection{Output Impedance of the Power Amplifier}

An ultra-low output impedance RF power amplifier for 3T MRI systems has been developed by a group in GE Global Research Center to simultaneously deliver maximum power to the load and improve the inter-coil isolation of a transmit array [8]. This method resembles the preamplifier decoupling approach for receive coil arrays [2], where the matching networks for receive coils behave like parallel resonant circuits. They transform the low input impedance of the preamplifiers to a high impedance at the coil, thus limiting the current flowing in each coil and the inter-coil isolation is improved. In [64], a detailed explanation of the mechanism of construction of a four-channel receive array is available. The concept of the preamplifier decoupling approach is shown in Fig. 3.31. When the PIN diode $D 1$ is conducting, a fixed capacitor $C 1$ with an inductor $L 1$ are used to form a parallel resonant circuit in order to create high impedance in series with the coil, thus decoupling the receive coil during the transmit mode. Capacitor $C_{\mathrm{DC}}$ is used as a DC block. A fuse is place in the coil loop as a safety precaution in case a high current flows in the coil due to a failure in the active detuning circuit during transmit. In receive mode, the coil should be tuned to resonance by using the fine tuning capacitor $C_{\mathrm{T}}$ and matched to the optimal noise match of the preamplifier (usually $50 \Omega$ ) using $C_{\mathrm{M}}$. The coaxial cable, including the choke balun, and $C_{\mathrm{ph}}$ transform the low preamplifier impedance to a parallel inductance across $C_{\mathrm{M}}$ for preamplifier decoupling. 


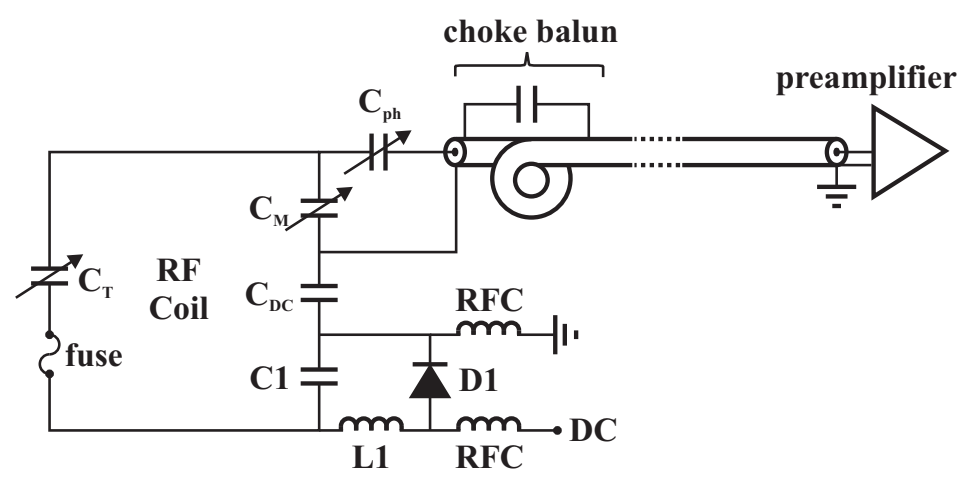

Figure 3.31: A schematic of a single receive element and preamplifier combination.

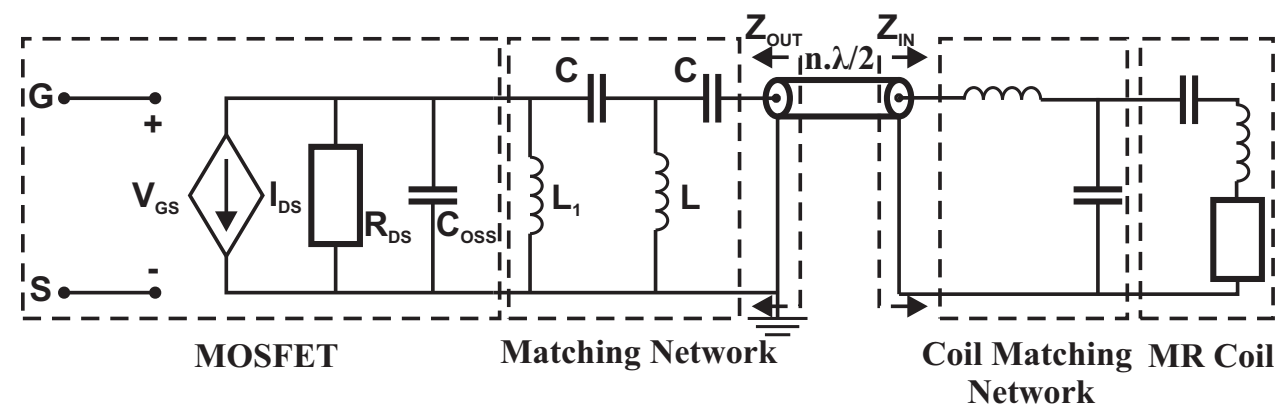

Figure 3.32: Ultra-low output impedance PA feeding a matched MR coil.

\section{The Concept of an Ultra-Low Output Impedance Power Amplifier}

In order to be able to apply a similar decoupling approach for parallel transmit, the power amplifier must present low impedance to the coil. The tuning strategy that has been followed in [8] is illustrated in Fig. 3.32. The power MOSFET can be modeled as voltage-controlled current source once it operates in the saturation region of its DC-characteristic. The current source $\left(I_{\mathrm{DS}}\right)$ is characterized by a high drain-source resistance $\left(R_{\mathrm{DS}}\right)$. The inductor $\left(L_{1}\right)$ resonates the drain-source capacitance $\left(C_{\mathrm{OSS}}\right)$ so the MOSFET's output presents a high impedance. The T-section matching network (C-L-C) is tuned for large signal power match while it transforms the drain-source resistance $\left(R_{\mathrm{DS}}\right)$ to a very low output impedance $\left(Z_{\mathrm{OUT}}\right)$. The coil matching network up-transforms the low coil impedance to $Z_{\mathrm{IN}}=50 \Omega$. With the low output impedance seen into the PA, the coil sees a parallel resonant circuit which minimizes induced coil current due to inter-coil coupling. In [9], we have proven the capability to operate our power amplifier in Fig. 3.16 as an ultra-low output impedance power amplifier by suitably tuning the matching network. The tuning strategy has been performed at the Larmor frequency for $7 \mathrm{~T}(298 \mathrm{MHz})$, and for the optimal operating point mentioned in section 3.6.4. The gate voltage of the final stage was fixed to $2 \mathrm{~V}$ to bias the drain current to $4.88 \mathrm{~A}$ at $50 \mathrm{~V}$ drain voltage. This bias setting allows the BLF188XR transistor to operate in the saturation region of its DC-characteristic. To calculate 


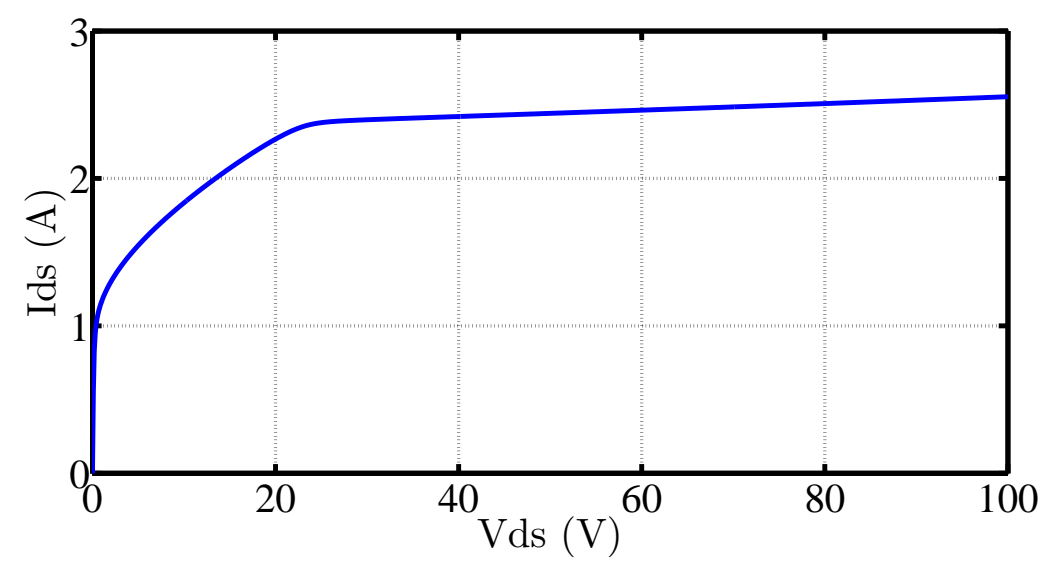

Figure 3.33: The DC output characteristic for single transistor of BLF188XR.

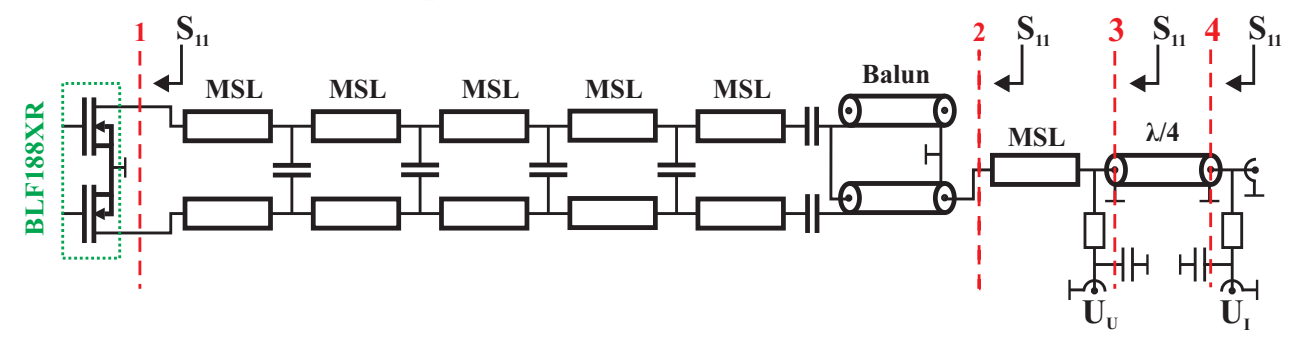

Figure 3.34: An output matching network in the last amplification stage.

the DC drain-drain resistance $\left(R_{\mathrm{DD}}\right)$, a DC output characteristic plot of the transistor is required. For a single transistor of the BLF188XR pair, $R_{\mathrm{DS}}$ can be obtained from Fig. 3.33 by calculating the inverse of the slope of the $I-V$ curve in the saturation region. The result that we obtain is $442.5 \Omega$. For both transistors of the BLF188XR pair, this result should be doubled. However, this result is valid only at DC, whereas the AC measurement result appears different. Based on an AC simulation, and when the power amplifier is powered, the output impedance of the transistor by measuring directly between both drain terminals at reference plane 1 in Fig. 3.34, $Z_{\mathrm{dd}}=0.261-\mathrm{j} \cdot 4.759 \Omega$ and the corresponding reflection coefficient is $0.99 \angle-169^{\circ}$, seen in Fig. 3.35. In [8], a shunt inductor $\left(L_{1}\right)$ is proposed at the output of the transistor to resonate the drain-source capacitance $\left(C_{\mathrm{OSS}}\right)$, thus the output of the transistor presents a high impedance. For our case this concept fails, since by adding a shunt inductor $(2.55 \mathrm{nH})$ to resonate the capacitive part, we only get a pure resistance value of $87 \Omega$ as shown in Fig. 3.35. Because of this relatively low impedance, an alternative matching concept than that proposed in [8] has been employed to fulfil the ultra-low output impedance amplifier requirement.

In Fig. 3.34, a multistage matching network in combination with a symmetrical balun transforms the coil impedance (nominally $50 \Omega$ ) to the optimum value for large signal power transfer. In the opposite direction, it transforms the $\mathrm{AC}$ drain-drain impedance $\left(Z_{\mathrm{dd}}\right)$ seen at 


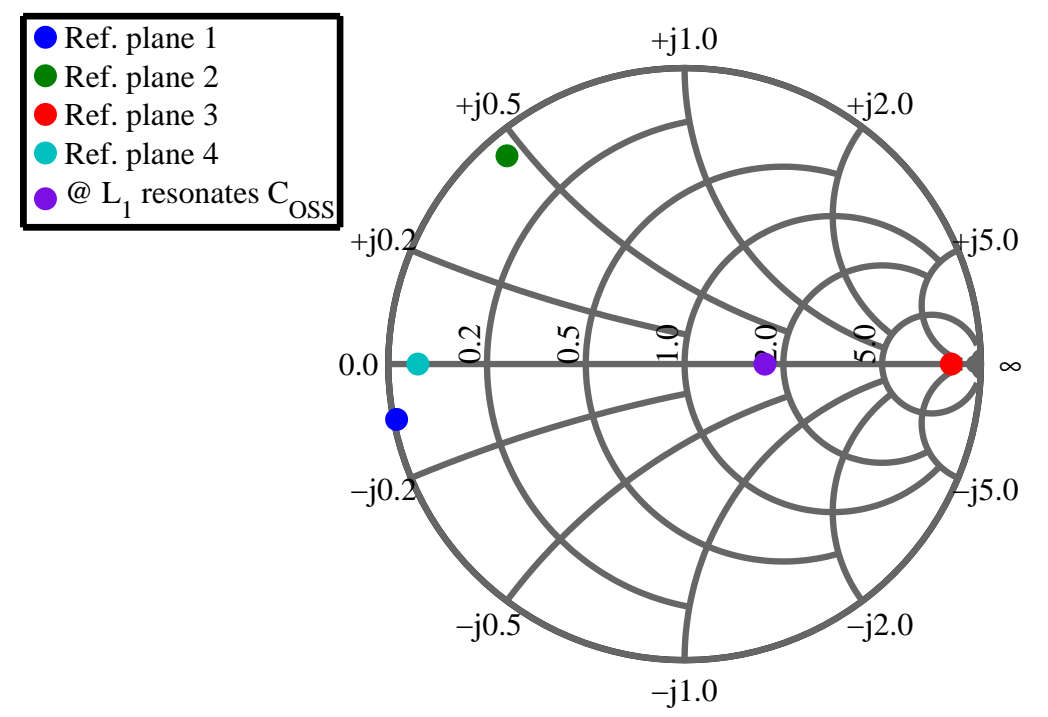

Figure 3.35: Reflection coefficients for reference planes 1-4.

reference plane 1 to an impedance which has a reflection coefficient of $0.924 \angle 130^{\circ}$ at reference plane 2. This results from transformation of $Z_{\mathrm{dd}}$ by the matching network which behaves like a low impedance transmission line with ca. $\lambda / 12$ electrical length. A $50 \Omega$ microstrip line has been added after the balun to provide an electrical delay so the output impedance seen at reference plane 3 is high with corresponding reflection coefficient of $0.90 \angle 0^{\circ}$, seen in Fig. 3.35. A quarter-wave transmission line is responsible to turn the high output impedance into a low output impedance with corresponding reflection coefficient of $0.90 \angle 180^{\circ}$ at reference plane 4 . This matching and electrical delay concept ensures simultaneously a maximum power transfer and very low output impedance for inter-coil isolation.

\section{Load Dependence Characterization}

In conventional MR systems, circulators are used in the transmit chains between the power amplifiers and the transmit coil elements in order to isolate the power amplifier from any reflected wave which might appear due to load mismatch or mutual coupling. To evaluate the load dependence of our amplifier, a comparison between two transmit chain setups is performed. Fig. 3.36(a) shows a setup with circulator while Fig. 3.36(b) shows a direct connection setup. Both setups employ a variable load impedance to emulate either mismatch or mutual coupling phenomena. For an extreme case of coupling that might occur in parallel excitation systems where the coupling into neighbor array coils can be very high, the reflection coefficient $(\Gamma)$ of the variable load has been selected to vary between -2 and +2 . In the following study, and 
for large signal operation, our amplifier is driven to deliver $500 \mathrm{~W}$ peak power to a $50 \Omega$ load corresponding to a peak output voltage $V_{\text {out }}$ of around $225 \mathrm{~V}$. In order to obtain an isolation between array coils by suppression of induced current, the PA should behave like a voltage source (low output impedance), meaning that the output voltage value should be maintained for all load impedance values. Fig. 3.37(a) shows the output voltages for both setups by sweeping the real reflection coefficient. The first observation from these two curves is that both curves

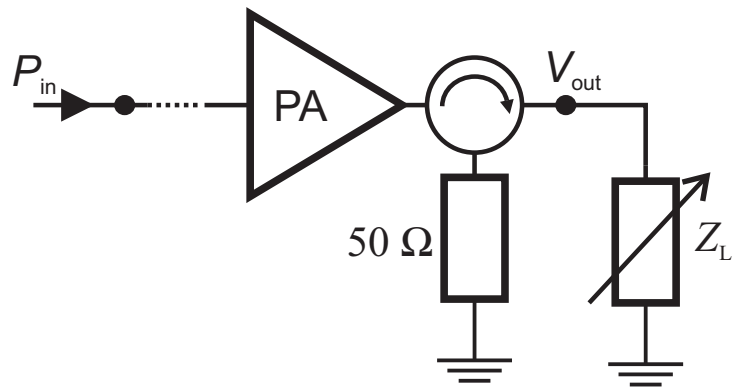

(a)

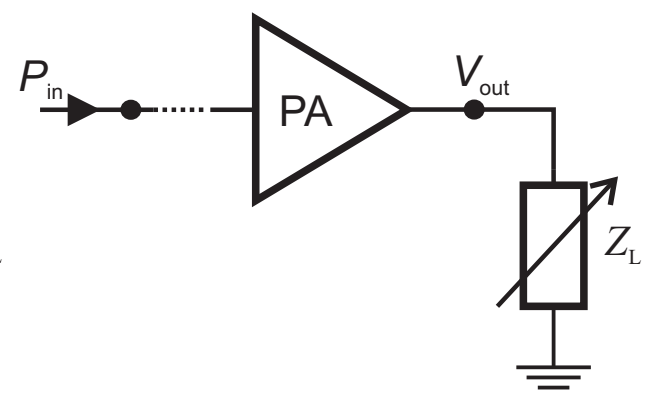

(b)

Figure 3.36: (a) Conventional transmit chain setup, (b) connection without circulator.

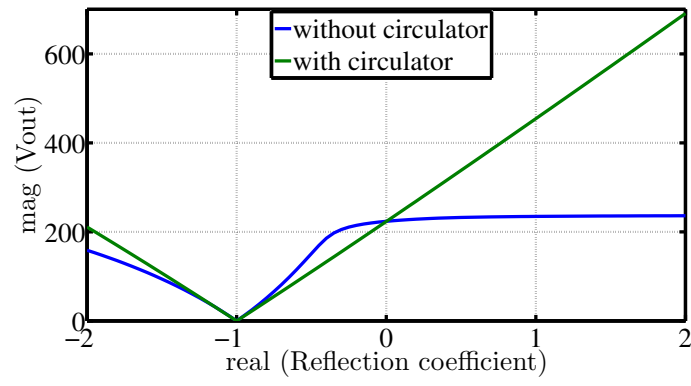

(a)

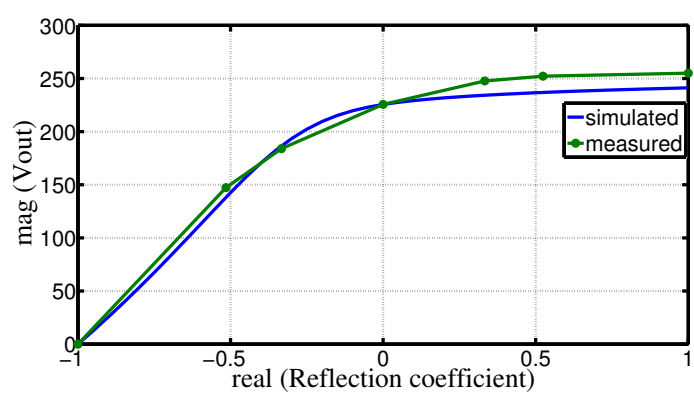

(b)

Figure 3.37: (a) Magnitude of output voltage for both setups under the sweep of the real reflection coefficient between -2 and 2, (b) simulated and measured output voltage at $500 \mathrm{~W}$ peak power (at $50 \Omega$ load) for direct connection setup under the sweep of the real reflection coefficient between -1 and 1 .

naturally intersect in two points : when $\Gamma=0$ (matched load), and when $\Gamma=-1$ (short circuit load). When $-1<\Gamma<0$, the power amplifier in direct connection setup tries to push up the output voltage levels to reach the matched load level and this provides more constant output level than the conventional setup. For $\Gamma>0$, the increase of the output voltage over the matched load level is negligible while in the conventional setup the output voltage increases with the reflection coefficient, as to be expected from a generator of $50 \Omega$ impedance. For $-2<\Gamma<-1$, the direct connection setup has no advantages over the conventional setup. These results prove that our PA 
design, to a good approximation, and over a wide range of impedances behaves like a voltage source with much lower impedance than $50 \Omega$. Fig. 3.37(b) compares a test result obtained by driving the prototype PA to deliver $500 \mathrm{~W}$ peak power to $50 \Omega$ load under the sweep of the real reflection coefficient between -1 and 1 . A good agreement between simulated and measured results is noticeable.

On the other hand, the PA design exhibits dependencies on the load impedance as to be expected. Fig. 3.38(a) shows the output power as a function of the load impedance. We realize that the saturated power will be less than what is generated at $50 \Omega$ for any other load; what may also be important for an application is the variation of the amplifier gain below the saturation (the lower the load impedance, the larger the gain) and the variation in the compression level (the lower the load impedance, the lower the input compression level). This characteristic is not seen in PAs with circulator output and may make the excitation control of a Tx array difficult. In the our target application, however, it is the PA output voltage which has to be controlled. As seen in Fig. 3.38(b), the gain variation below the compression level is much reduced when the output voltage is compared for different load impedances due to the low output impedance. But still the compression levels vary with the load and the output voltage varies strongly with the load impedance once the PA is driven into deep saturation. This characteristic would limit the application of our PA to low and medium drive power levels if no compensation technique is applied, like the feedback control, as described in $\mathrm{Ch}$. 5.

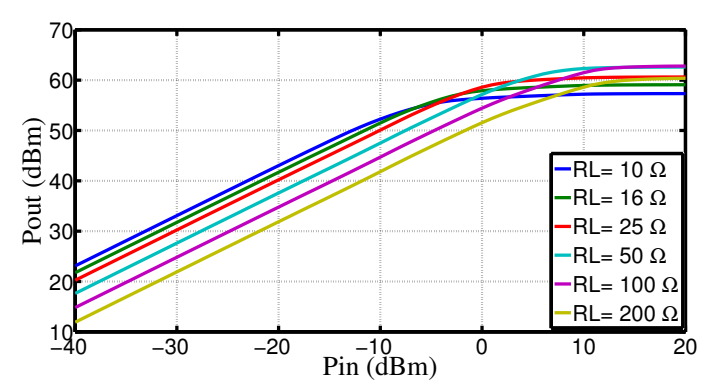

(a)

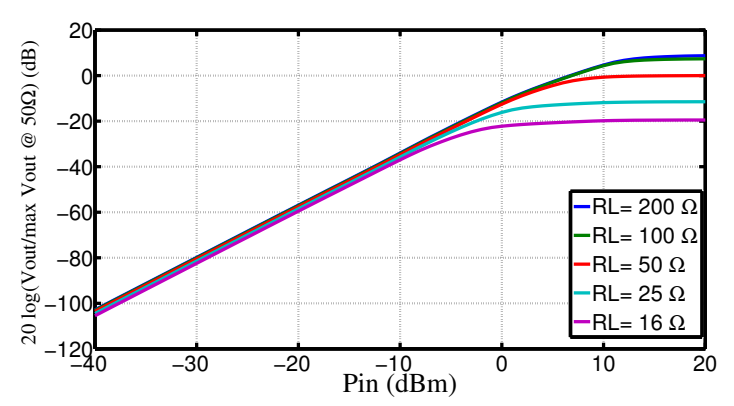

(b)

Figure 3.38: PA load dependence of (a) output power, (b) output voltage.

\section{Coil Decoupling Performance by our Amplifier}

The decoupling of the coils by presenting an ultra-low impedance by the connected PA was tested experimentally and by simulation. In our measurement, a meander coil with high-dielectric substrates and a quarter-wave balun transformer has been employed as shown in Fig. 3.39. A pair 
of geometrically decoupled pick-up loops placed over the meander coil measures the isolation provided by different types of load (open, short, $50 \Omega$ ) and in addition by loading the coil with the PA output (the PA powered but inactive). From Fig. 3.40, we can see the coupling due to the PA termination to be similar to the behaviour of the short circuit termination. We can observe that the highest coupling occurs by open circuit (O.C.) termination as this permits the highest current to flow in the coil. The lowest coupling is seen by short circuit (S.C.) termination because this produces infinite impedance in series with the coil impedance, prohibiting any current to flow. In Table 3.2, coupling results are normalized to the case of the matched termination to produce a measure of isolation of coils from neighbouring coils. As a result, we find our PA allows an improvement of $18.7 \mathrm{~dB}$ over the conventional $50 \Omega$ termination.

The performance of PA decoupling has also been investigated by EM-simulation of two coupled meander coils as seen in Fig. 3.41, where the first element (on the left side) is fed by

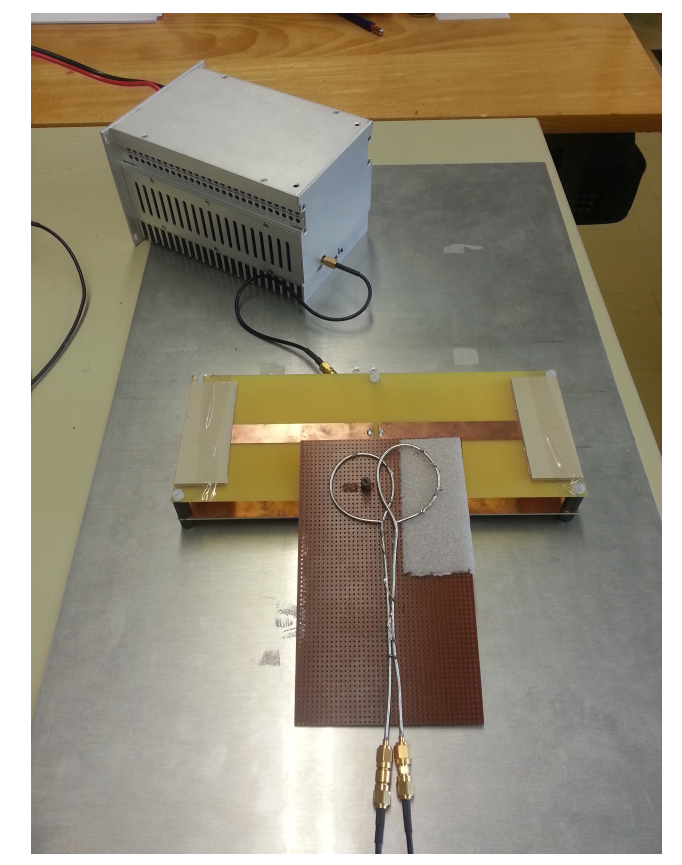

Figure 3.39: Coupling measurement setup using two decoupled pick-up loops placed over a meander coil terminated by the PA.

Table 3.2: Isolation measured for different types of termination.

\begin{tabular}{|c|c|}
\hline Termination & Isolation $(\mathrm{dB})$ \\
\hline S.C. & 32.8 \\
\hline PA & 18.7 \\
\hline O.C. & -5.23 \\
\hline $50 \Omega$ & 0 \\
\hline
\end{tabular}




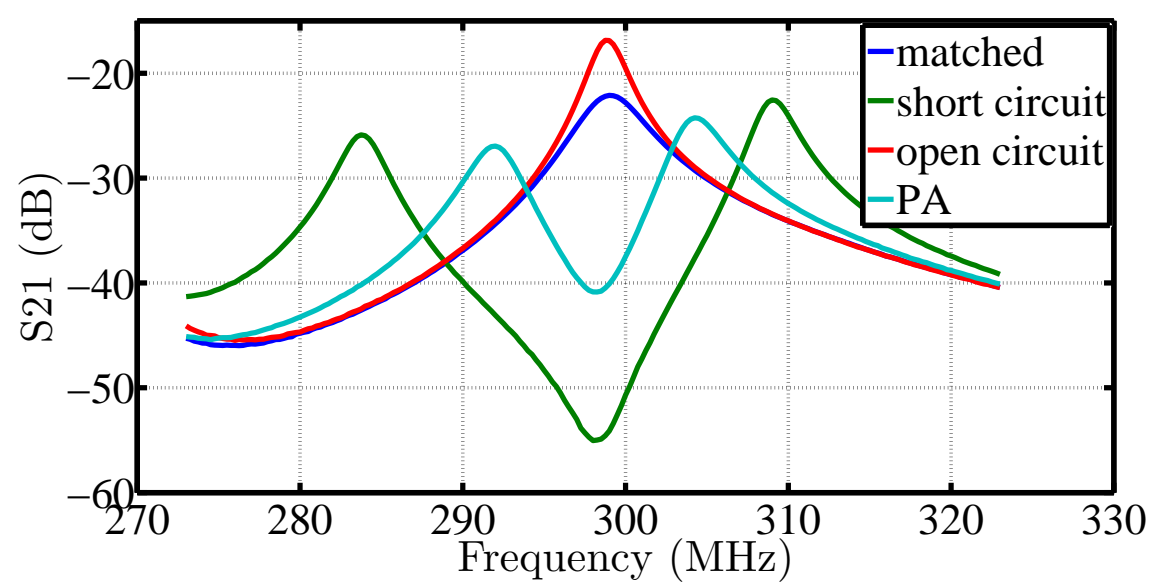

Figure 3.40: Measured coupling through coil with different coil terminations.

$0.5 \mathrm{~W}$ accepted power while the second element (on the right side) is either matched terminated as in Fig. 3.41(a) or terminated by the PA output reflection coefficient as in Fig. 3.41(b). Numerical simulation was performed using a FDTD tool (CST Microwave Studio) by simulating two parallel aligned meander coils with $100 \mathrm{~mm}$ gap distance and $200 \mathrm{~mm}$ below a homogeneous phantom $\left(\varepsilon_{r}=45.3, \sigma=0.8 \mathrm{~S} / \mathrm{m}\right)$. Due to high coupling between the two meander coil elements $\left(S_{21}=-9.61 \mathrm{~dB}\right.$ in the case of $50 \Omega$ termination), strong induced current flows in the coupled element, producing strong magnetic field, as seen in Fig. 3.41(a). Contrary, Fig. 3.41(b) shows a much smaller field around the coupled coil element when it is terminated by the PA.

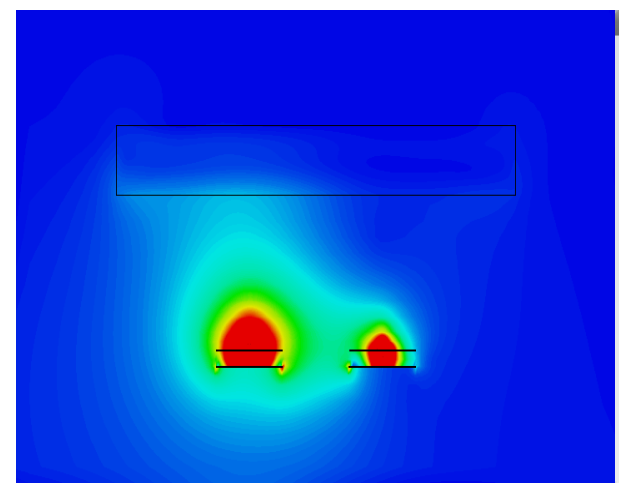

(a)

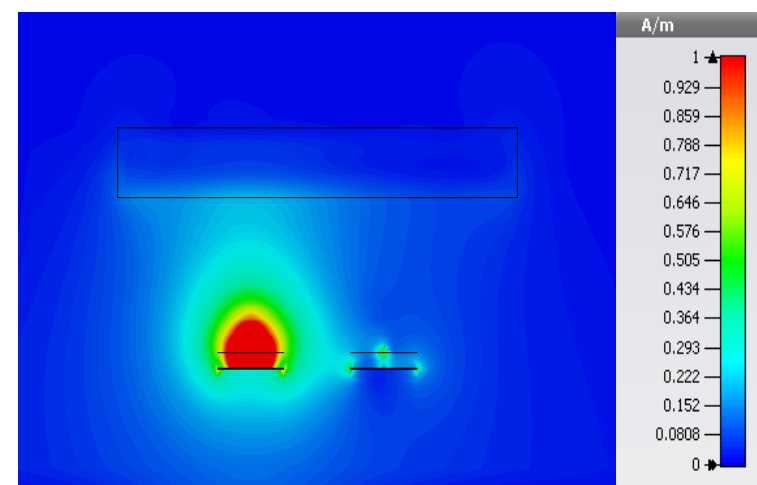

(b)

Figure 3.41: $|H|$ in mid-transversal section for coupled meander coils as seen in FDTD simulator. (a) The second element is matched terminated, (b) the second element is terminated by the PA output impedance. 


\section{Output reflection coefficient of power amplifier}

Characterizing a linear device using small signal S-parameters is considered as a trivial issue for RF circuits designers. By moving to characterize nonlinear devices especially when they are excited with high power, special considerations are required concerning the measurement concept. For example, high power amplifiers have different load lines (e.g., load line that allows maximum power, maximum gain, maximum efficiency, etc.) between which the designer might choose depending on the particular application of the amplifier. In addition, the optimum load impedance of a power amplifier varies with the RF excitation level. A meaningful measurement for the output impedance (i.e., output reflection coefficient) should be done while applying a realistic drive level to the input of the power amplifier. Such measurement is known as "hot S22". Measuring "hot S22" is quite difficult because the power produced by the power amplifier maybe high enough to damage the network analyser. Additionally, a discrimination between the reflected output signal and the amplified drive signal should be obtained. This can be done once the "hot S22" measurement is made with a frequency that is slightly offset from the drive signal frequency. Fig 3.42 shows the simulation setup for "hot S22" which utilizes the "sniffer" circuit ( an ideal four-port circuit, non-realizable because it breaks the conservation of energy rule). It has zero loss, zero reflection and zero phase shift between the ports. An incident signal on port 1, will exit port 2 and port 3 equal in phase and amplitude. An incident signal on port 2 , will exit port 1 and port 4 equal in phase and amplitude. Thus the reflection coefficient at port 1 can be obtained from the ratio $V_{\mathrm{P} 3} / V_{\mathrm{P} 4}$. This simulation setup allowed us to study the PA output large-signal reflection coefficient $\left(\Gamma_{\mathrm{PA}}\right)$. In the first case, the PA's input port is matched terminated (inactive mode) and the PA is powered, whereas a reverse power $\left(P_{\text {rev }}\right)$ enters into the output port from an external source (emulating the coupling from a neighbour coil). In the

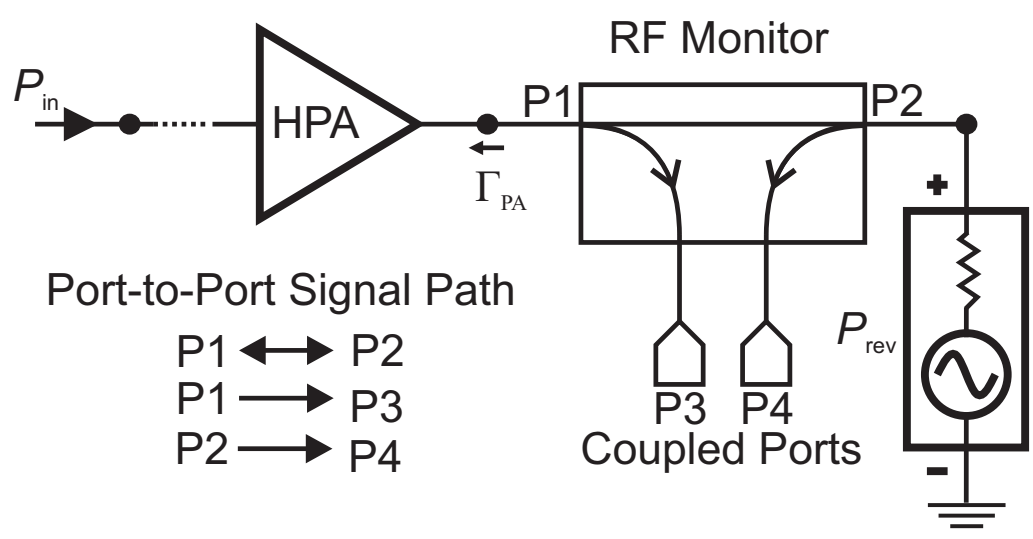

Figure 3.42: Measurement setup for "hot S22". 


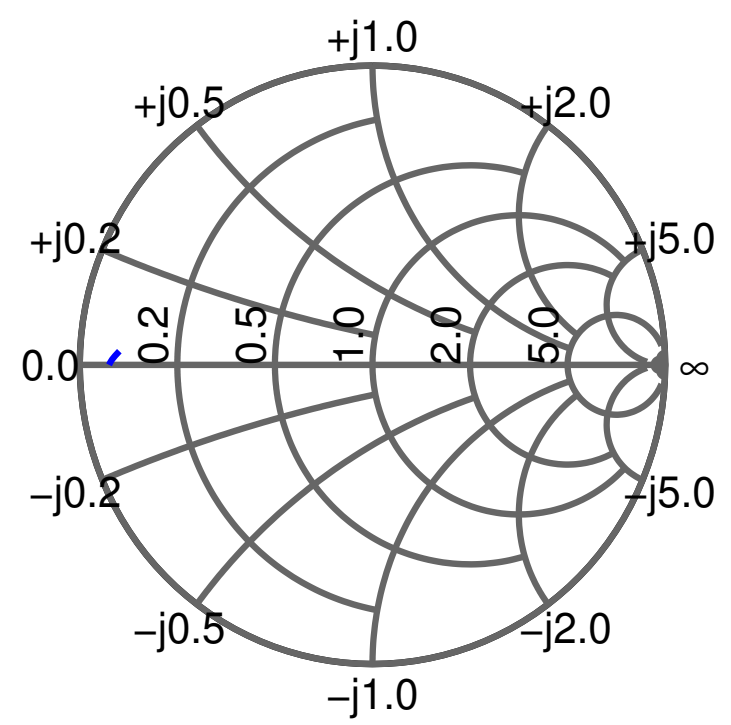

(a)

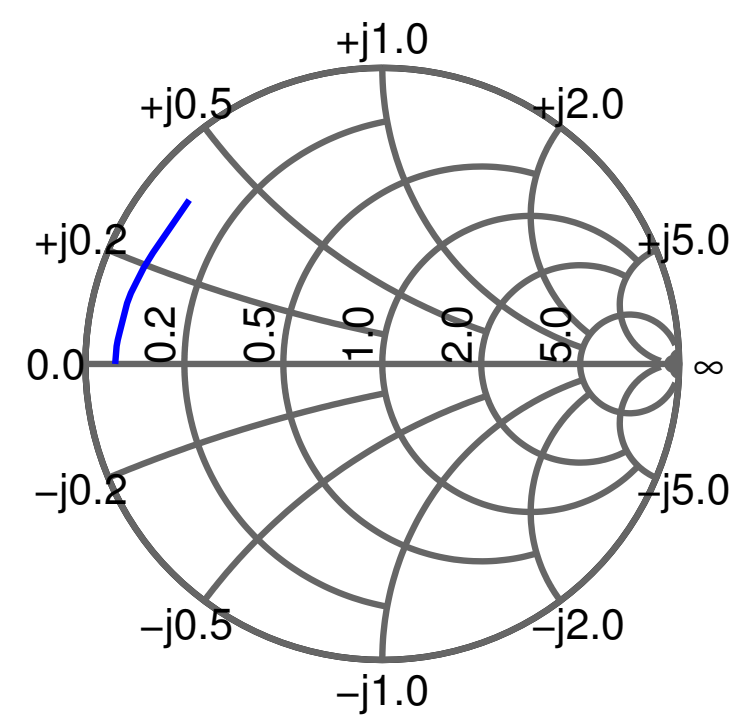

(b)

Figure 3.43: Simulated PA reflection coefficient $\left(\Gamma_{\mathrm{PA}}\right)$ as function of (a) reverse power $(0 \mathrm{~W}$ $\longrightarrow 200 \mathrm{~W})$, (b) forward power $(0 \mathrm{~W} \longrightarrow 1 \mathrm{~kW})$.

second case, the PA is driven (active mode) and delivers power to the load $\left(P_{\mathrm{fwd}}\right)$. Fig.3.43 shows that the reflection coefficient $\left(\Gamma_{\mathrm{PA}}\right)$ for the PA remains high, while the phase shifts when delivering power to a matched load or when receiving reverse power.

\section{Effect of lossy cable on PA Decoupling Performance}

In section 3.6.2, we have discussed the influence of lossy (multiple) half-wave cable on the reflection coefficient seen at the other end of the cable. Fig. 3.44 shows the same influence on the output reflection coefficient of PA where the reflection coefficient converges approximately to zero by connecting a cable length of $100 \lambda$. This degradation in the output reflection coefficient will be reflected in the decoupling performance of the PA. Thus, the inter-coil isolation will decay as the cable length increases. In our 7T MRI installation at the Erwin L.Hahn Institute, a cable length of about $5 \mathrm{~m}$ (about $7.5 \lambda$ ) has been employed such that the degradation of PA decoupling was acceptably low. 


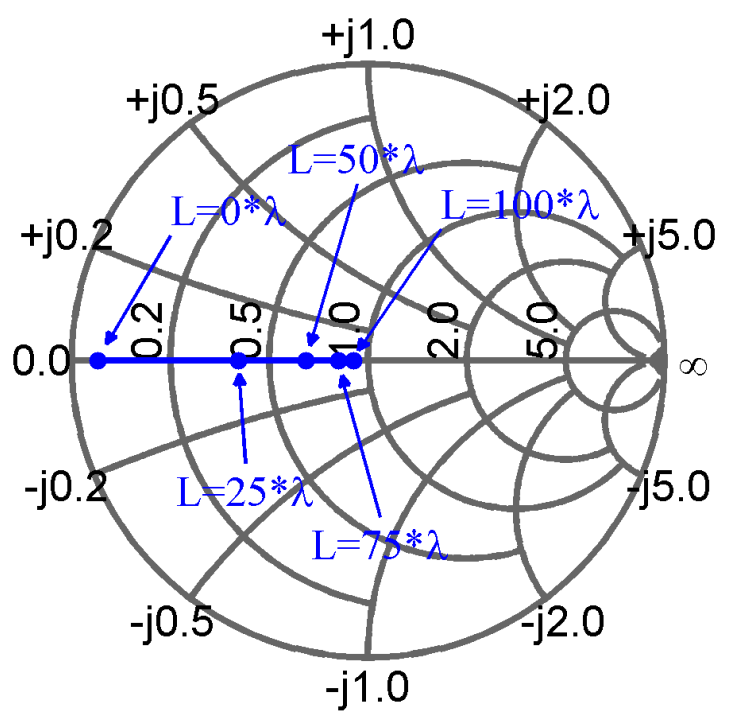

Figure 3.44: The output reflection coefficient of HPA calculated after lossy (multiple) half-wave cable in the range between $0 \lambda$ and $100 \lambda$. 


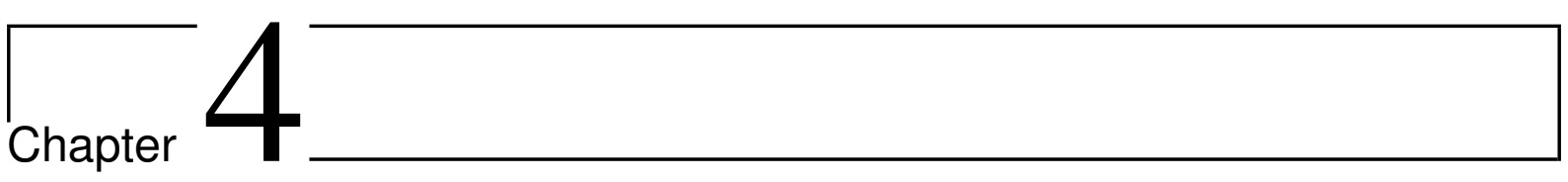

\section{FEEDBACK SYSTEM}

\subsection{Introduction}

Since positive feedback tends to lead to instability, negative feedback is used instead because it tends to stabilize the system by reducing the fluctuation in the output. Negative feedback is extensively used in mechanical (e.g., a thermostat in the heater) and electronic (e.g., op-amp with negative feedback) engineering. It can be seen also in physical system such as the climate. Biological negative feedback is also existing within living organisms where several physiologic parameters (e.g., human body temperature or human blood pressure) are controlled.

In communications, the demand of highly linear RF power amplifiers increases to be used in several applications such as radio and TV transmitter, wireless communications and satellite communication system. In [50], a linearization technique using the feedback method is demonstrated in its different categories: RF feedback, envelope feedback, polar feedback, and Cartesian feedback. The method is used to address the distortion problems introduced by RF power amplifiers and improve its linearity. Once a feedback is applied to a high efficiency amplifier, the trade-off between linearization and efficiency is relaxed.

In MRI systems, feedback loops have been widely used to address the problem of array coil interactions during transmission [12], [13]. The feedback loops are designed to reduce the induced currents generated due to mutual coupling between the elements in coil array.

Feedback loop systems should be designed carefully to overcome any possibility of instability. A sufficient phase margin is highly desirable in feedback systems to fulfil the Bode stability criterion even when unwanted phase changes are introduced in the loop. Once the loop gain of a feedback system is one or more by the frequency at which the phase shift of $180^{\circ}$ has been 
achieved, the negative feedback becomes positive feedback and the amplifier will become an oscillator and produce unwanted signal.

\subsection{A brief history of Feedback}

In the context of electronic amplification, the term "negative feedback" was first used in September 1933 by Alan Blumlein's and Henry Clark's patent [65], which specifies the use of a negative feedback loop in an amplifier. They outlined a method that combines both current and voltage feedback. This patent pre-dates Black's negative feedback patent [66] which outlined the method in January 1934. He describes and explains the theory of the feedback principle and demonstrates how stability of amplification and reduction of modulation products follow when stabilizing feedback is applied to an amplifier. In the early 1930's, Harry Nyquist of Bell Labs discovered the general condition under which a negative feedback amplifier becomes stable [67]. His criterion generally is called "Nyquist stability theorem", which is one of the most general stability tests for designing and analyzing systems with feedback. The earliest published literature on feedback amplifiers and stability investigation, at least to the knowledge of the author, was in 1945 [68]. Voyce [69] implemented an IF (Intermediate Frequency) feedback for linearizing a power amplifier. This technique allows loop compensation and added loop gain to be performed at the intermediate frequency. The polar loop transmitter was introduced by Petrovic in 1979 [70]. His transmitter has polar feedback which utilizes the magnitude and the phase of the power amplifier output signal as feedback signal to address the distortion problems introduced by the PA. In the early 1980's, Petrovic invented the Cartesian feedback loop which uses inphase and quadrature signals for the same purpose [71].

In 1989, the method of Cartesian feedback was first mentioned in the context of magnetic resonance under the title of quadrature feedback [72], while the first utilization in an MR system was in 2004 by Hoult [10,11], to address the interactions between the phased-array coils. In 2008, he demonstrated the blocking capability by increasing the gain-bandwidth product (GBWP) of the feedback loop [13], whereby unwanted induced currents can be blocked efficiently.

\subsection{Basic Feedback System}

Consider a basic negative feedback system shown in Fig. 4.1. If any changes occur at the system output, the negative feedback will counteract this change by affecting the input. The forward 


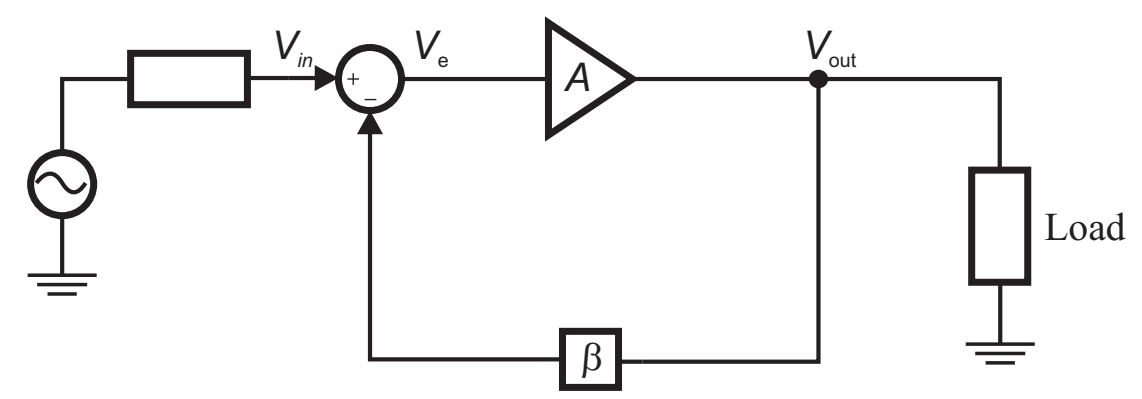

Figure 4.1: Direct negative Feedback loop.

path consists of an amplifier of gain $A$ and the feedback path consists of voltage divider of gain $\beta$. The comparator subtracts the feedback signal from the input signal to form the error signal $V_{\mathrm{e}}$ which drives the system. The error signal will be amplified to produce a system output signal $V_{\text {out }}$ :

$$
V_{\text {out }}=V_{\mathrm{e}} A
$$

The error signal is defined by

$$
V_{\mathrm{e}}=V_{\text {in }}-\beta V_{\text {out }}
$$

Substitute Eq. 4.2 into Eq. 4.1

$$
\frac{V_{\text {out }}}{A}=V_{\text {in }}-\beta V_{\text {out }}
$$

Rearranging Eq. 4.3

$$
V_{\text {out }}\left(\frac{1}{A}+\beta\right)=V_{\text {in }}
$$

From Eq. 4.4, we can obtain the closed loop gain

$$
A_{C L}=\frac{V_{\text {out }}}{V_{\text {in }}}=\frac{A}{1+A \beta} .
$$

When the closed loop gain approaches $\infty$, the loop becomes unstable. This occurs when the loop gain $A \beta$ is -1 (i.e., $|A \beta|=1$ and $\angle(A \beta)=180^{\circ}$ ), or when $1+A \beta$ is 0 . Minimizing the error signal requires large loop gain due to their inverse proportionality

$$
V_{\mathrm{e}}=\frac{V_{i n}}{1+A \beta} \text {. }
$$




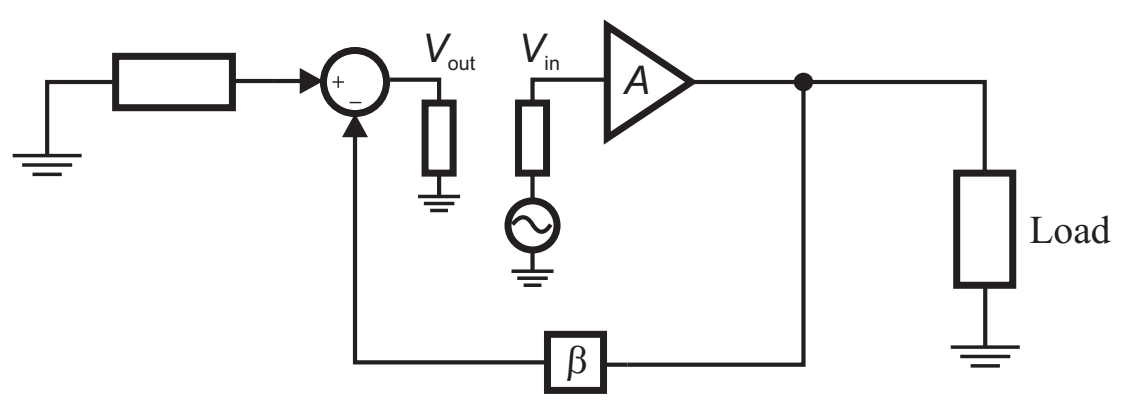

Figure 4.2: Return ratio test circuit.

In order to test the stability of a feedback system, the Return Ratio $(R R)$ method was suggested to be used [73] by which the "open" loop gain frequency response can be obtained. To apply this method, we need to replace all independent sources by their internal impedance. Then the feedback loop has to be broken between the source and the rest of the circuit to insert an independent test source. A test signal is injected to drive the circuit whereas the other end of the break is terminated by the equivalent impedance seen in the original circuit at this breaking point. Then the return ratio can be found by calculating the ratio of the signal measured at the equivalent impedance to the injected test signal. From Fig. 4.2, the $R R$ can be determined by the following:

$$
R R=\frac{V_{\text {out }}}{V_{\text {in }}}=-A \beta
$$

As is obvious from the equation above, the system's loop gain is the negative of the Return Ratio. After plotting the loop gain frequency response, we can determine the phase margin simply by measuring the phase difference between $\angle A \beta\left(f_{0 d B}\right)$ and $-180^{\circ}$ where $f_{0 d B}$ is the frequency at which $|A \beta|=0 \mathrm{~dB}$. The gain margin can also be determined by measuring the difference between $\left|A \beta\left(f_{180^{\circ}}\right)\right|$ and $0 \mathrm{~dB}$ where $f_{180^{\circ}}$ is the frequency at which $\angle A \beta=-180^{\circ}$.

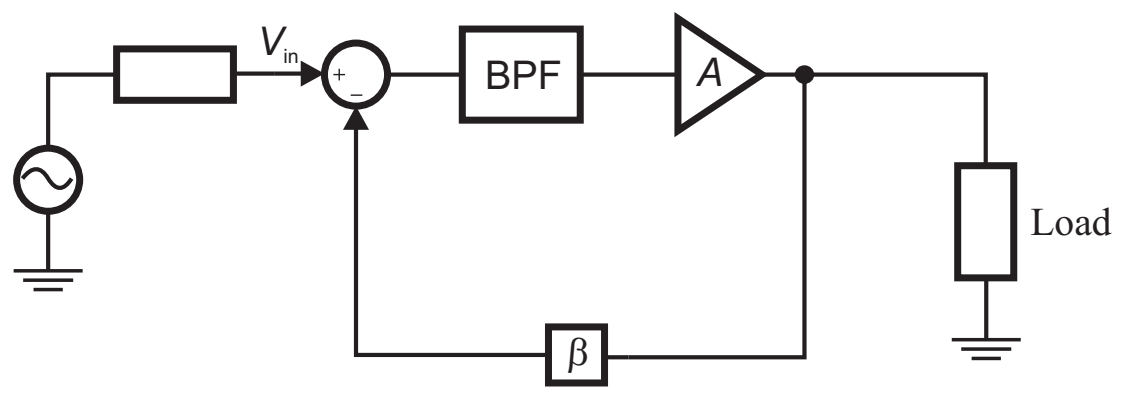

Figure 4.3: Direct negative FBL with BPF.

At VHF frequencies and in the microwave region, the use of direct negative feedback requires 

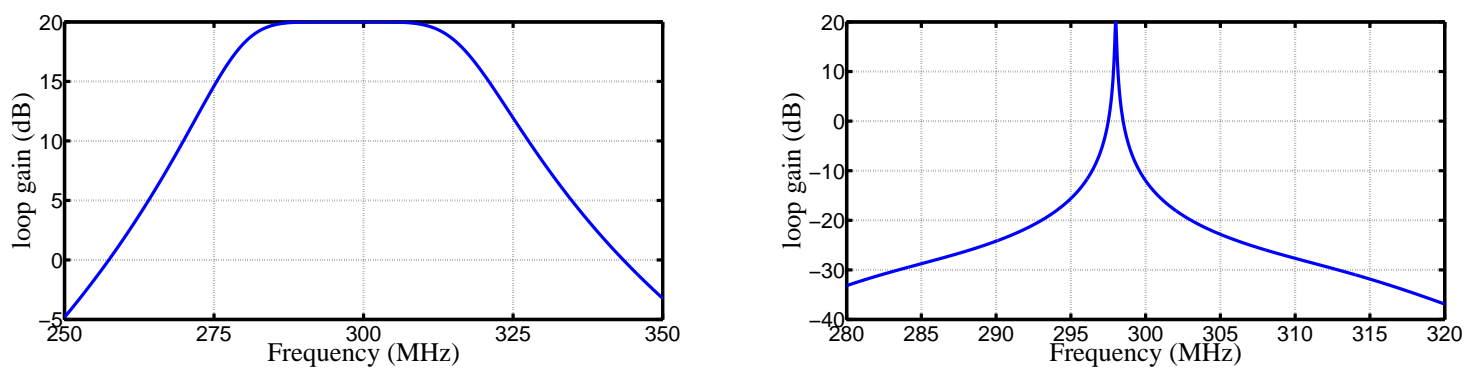

(a) loop gain magnitude
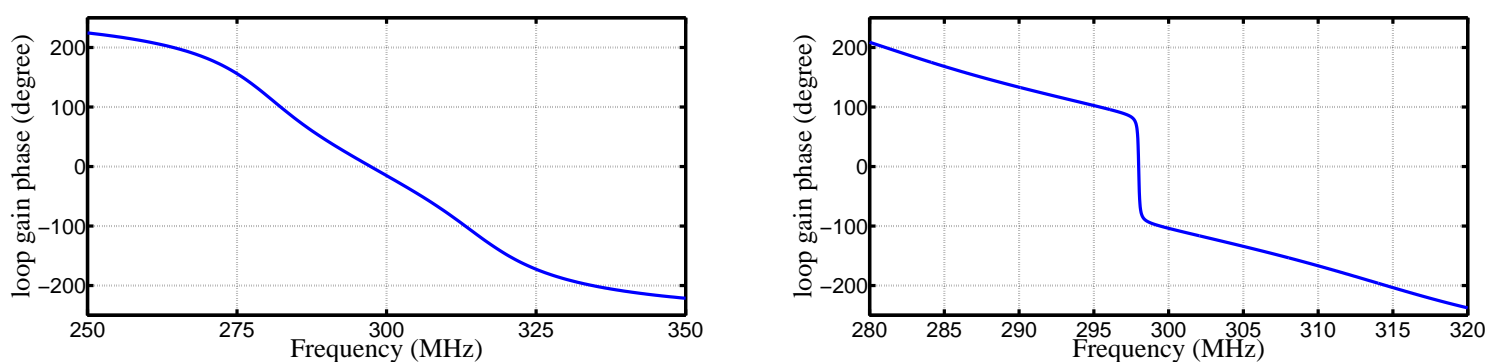

(b) loop gain phase
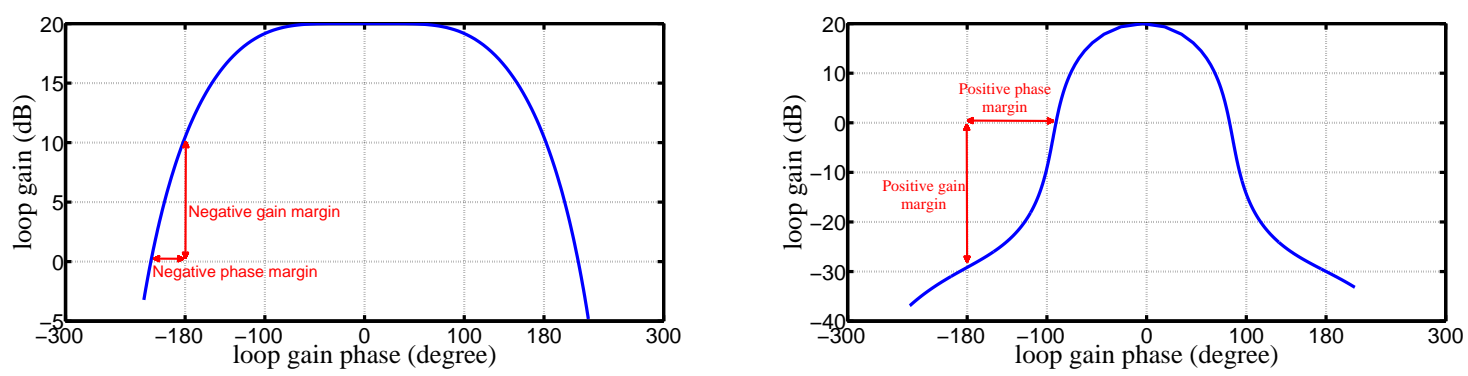

(c) magnitude vs phase

Figure 4.4: Stability analysis diagrams for: direct negative Feedback loop (left) and direct negative Feedback loop with BPF (right).

a considerable care from the circuit designers to stabilize the feedback loop. The forward path $A$ has a phase characteristic which can vary rapidly with frequency. The phase of the feedback path $\beta$ is adjusted to ensure subtraction at the operating frequency. However, unstable conditions have a good chance to be fulfilled leading to oscillation at a nearby frequency: Consider a direct negative feedback loop with loop gain of $20 \mathrm{~dB}$ which employs a power amplifier with operating frequency $298 \mathrm{MHz}$. When the occupied bandwidth by the loop is primarily determined by the power amplifier, this can be around several tens of MHz. However, due to several amplifier stages with several reactive components, the amplifier may exhibit considerable delay and its transfer phase may vary rapidly versus frequency, and a negative gain margin and a negative phase margin are obtained from the loop gain diagram of the open loop transfer function, as shown in Fig 4.4(left), leading to instability. In addition, the transmission lines required in a 
feedback path may produce delay and phase variation comparable to that of the amplifier. To stabilize the loop, a band pass filter (BPF) characterized by very narrow bandwidth (usually a very small fraction of the operating frequency) is required to be inserted within the loop [74] as shown in Fig. 4.3. The BPF keeps the loop stable by reducing the loop gain to less than $0 \mathrm{~dB}$ by the frequency at which a phase shift of $180^{\circ}$ has been achieved. Thus, positive phase and gain margins are retained as shown in Fig. 4.4(right).

\subsection{Conventional Cartesian Feedback Loop}

Although utilizing a BPF in the direct negative FBL stabilizes the loop, it decreases the degree of freedom in the operating frequency. The Cartesian feedback loop overcomes the frequency restriction and the problem of delay in the RF feedback loop by downconverting the RF signals to the baseband frequency, where the narrow-band filtering is performed without restriction in the RF operating frequency. The basic concept behind this technique still is negative feedback. It employs two identical, decoupled feedback loops after separating the RF signal into two orthogonal components: in-phase $I$ and quadrature $Q$ components. The Cartesian coordinates of these baseband components explain the reason of its name. The comparison between the reference signal and the output signal is done either at baseband [75] or at RF frequency as seen in Fig. 4.5. The distorted PA outputs due to power amplifier non-linearity or load mismatch is sampled and compared to the reference signal. The error signal is quadrature down-converted. The resulting quadrature baseband signals are then amplified by operational amplifiers to adjust the loop gain and filtered by low pass filters to stabilize the loop by limiting the loop bandwidth. The loop gain is preferable to be as large as possible to reduce the residual error in the loop, but is limited by the loop stability. The larger the loop gain, the smaller the phase margin. The amplified error in its quadrature form is up-converted at the local oscillator (LO) frequency to form the control signal that drives the power amplifier. Once the loop is stable and works properly, the control signal represents the pre-distorted signal of the reference signal that is needed to compensate for the distorted PA output signal. The control signal can be expressed in terms of the quadrature signals as the following:

$$
C(t)=I(t) \cos \omega_{c} t+Q(t) \sin \omega_{c} t
$$

where $\omega_{c}$ is the RF carrier frequency. 


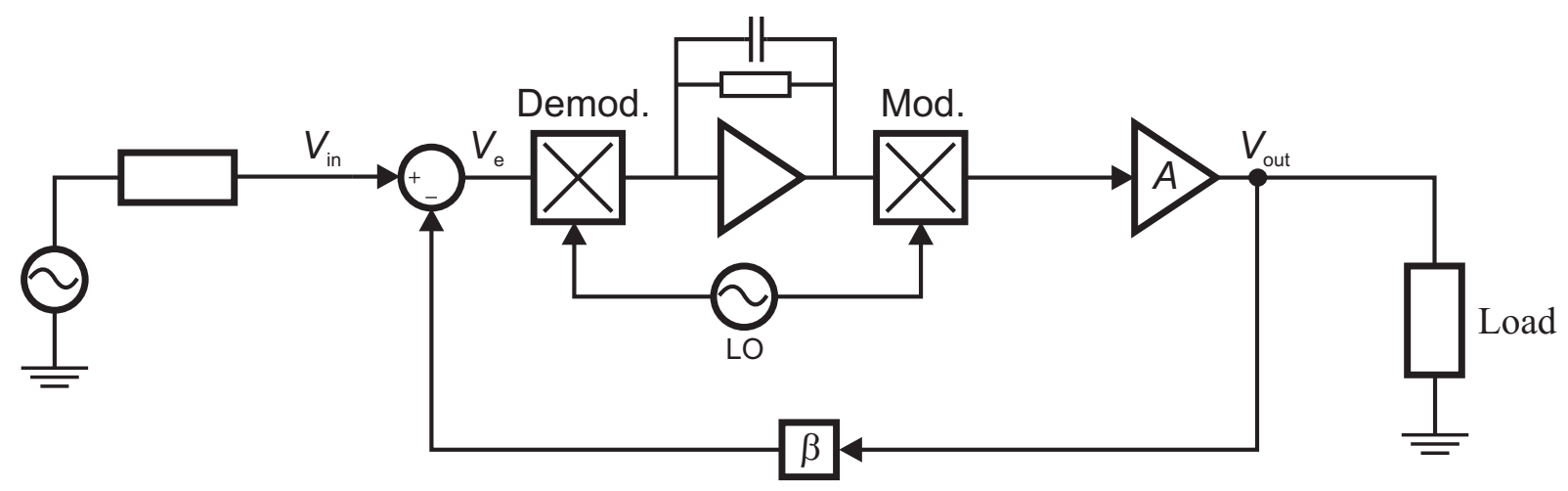

Figure 4.5: Conventional Cartesian Feedback loop with comparison at RF.

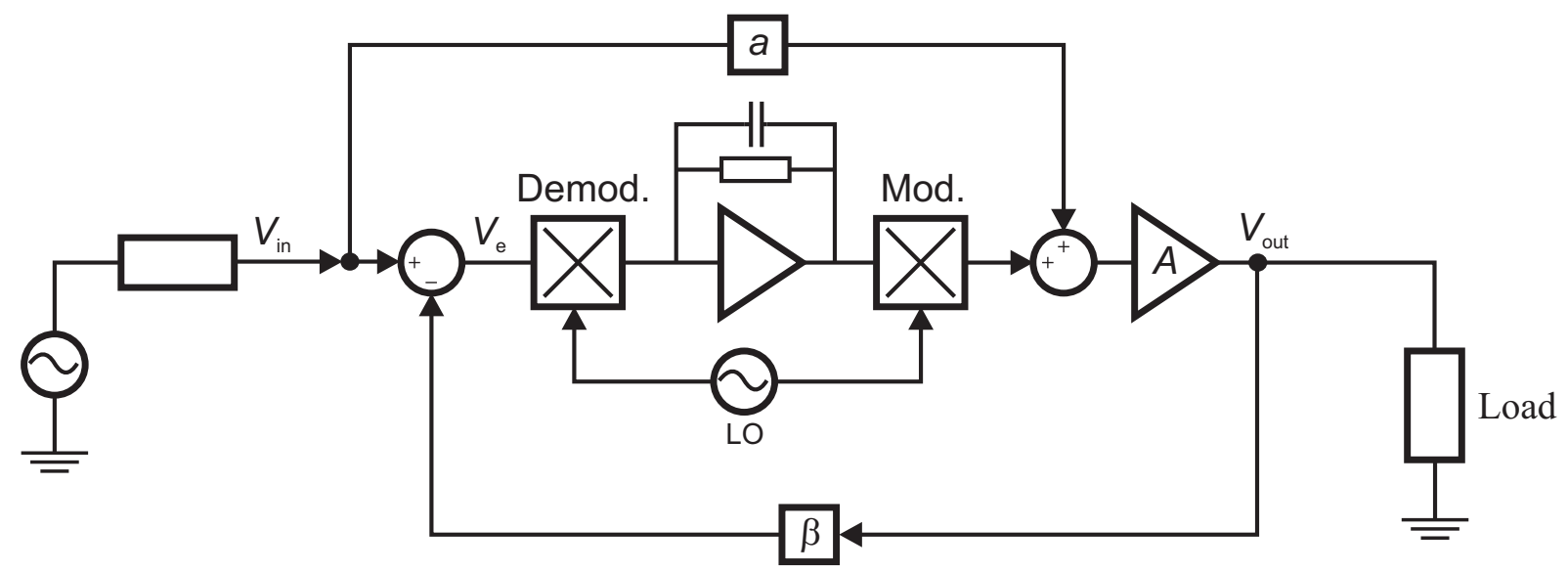

Figure 4.6: Conventional Cartesian Feedback loop with extra forward path.

In the Cartesian feedback loop, the down-converter and the up-converter are synchronised by splitting a common RF signal (LO). In practice, however, phase alignment between the reference signal and feedback signal requires a phase shifter to compensate for phase shift. Once the phase shifter is adjusted incorrectly, cross-coupling between the $I$ and $Q$ components occurs and the stability of the system is compromised. The impact of phase misalignment in Cartesian feedback systems has been studied by Dawson [76].

Another version of Cartesian feedback is seen in Fig. 4.6, which offers the flexibility of driving the power amplifier by an extra forward path whenever the feedback loop is unwanted. In case the feedback is used, the extra path drives the amplifier at a gain of $a \cdot A$ if the error signal $V_{\mathrm{e}}$ vanishes. This situation can be created by adjusting the feedback path $\beta$ for a given load (e.g., matched load) and at a certain power level (e.g., below compression). Only, when the amplifier is driven into saturation or the load impedance is changed, an error signal is created which adds a correcting signal at the amplifier input. The extra path can relax the loop gain requirement and thus improve stability as will be seen in Ch.5. 



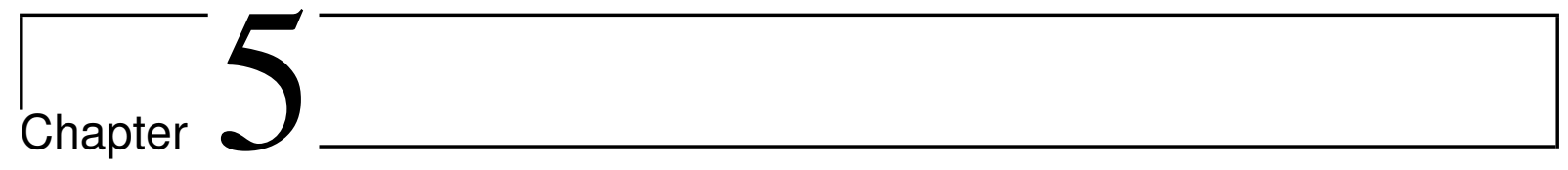

\section{UNCONVENTIONAL CARTESIAN}

\section{FEEDBACK}

\subsection{Concept of Unconventional Cartesian Feedback Loop}

The concept of the unconventional Cartesian feedback loop in Fig. 5.1 is a modification of the conventional Cartesian feedback loop with extra path as shown in Fig. 4.6. We see the extra forward path that drives the amplifier in case the error signal $V_{\mathrm{e}}$ vanishes. However, the second multiplier block (the modulator or up-converter) is driven by the I/Q baseband signal and the RF input signal instead of the fixed-amplitude LO. This leads to a major change in the loop gain characteristic as the up-converter gain becomes proportional to the RF input signal amplitude. This property of dynamic loop gain provides special advantages in the target application as described in the following sections.

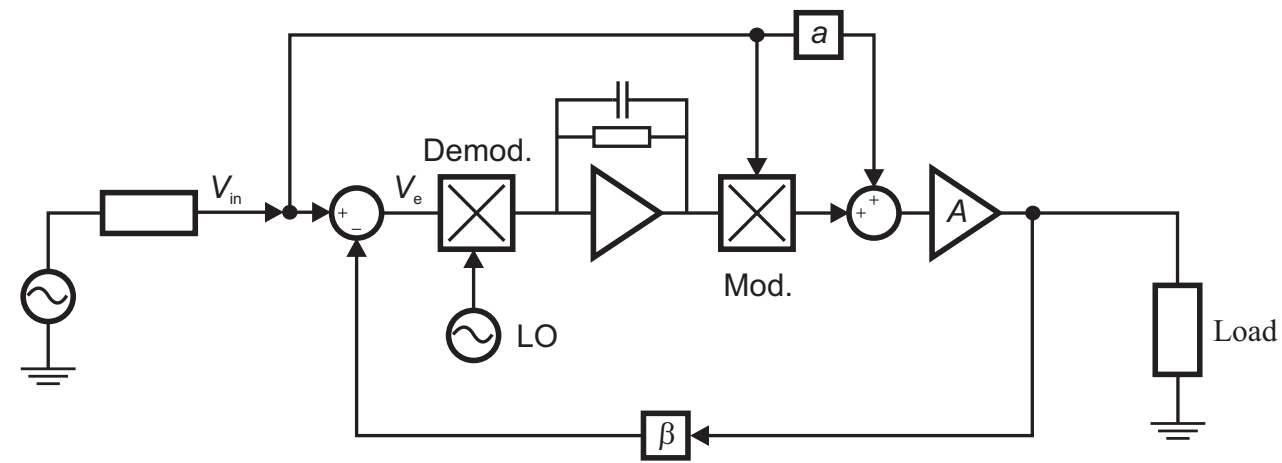

Figure 5.1: Unconventional Cartesian Feedback loop. 


\subsection{System Architecture and Simulation Setup}

The unconventional Cartesian feedback has been developed to be combined with the near-magnet pulse power amplifier (Fig. 3.16), which employs the concept of built-in coil current sensing, in order to control the current in the coil. In a conventional Cartesian FBL, a local oscillator (LO) is employed to drive the down-converter as well as the up-converter with constant signal level, whereas in our unconventional Cartesian FBL the LO is replaced by the RF input signal driving the down-converter through a limiter (i.e., with constant driving level) while the up-converter is driven dynamically according to the input signal level as seen in Fig. 5.2. The block diagram shows the amplifier system where an MR coil is connected to a $1 \mathrm{~kW}$ power amplifier (PA) by a $\lambda / 4$ coaxial cable. The geometry of the coil as well as several coil properties are explained in [40]. Based on the current forcing property that was explained in subsection (3.6.2), the coil current can be kept constant independent on mismatch due to the patient load or due to mutual coupling if the PA output voltage is controlled to be constant for a given input power. This can be accomplished by the feedback loop consisting of: IQ-Modulator (up-converter), IQ-demodulator (down-converter), active filters using differential amplifiers with 1st order low pass filters, phase shifter, attenuator and limiter. The D/A sets the modulator I/Q bias whereby it functions as the extra forward path in Fig. 5.1 setting the gain of this stage if no error signal has evolved. It is important to note that this setting has to be "backed off" from the maximum output power setting in order to allow a positive compensation (increase of gain) in the case that the output voltage is too low. The back off used in the simulations was set to $3 \mathrm{~dB}(500 \mathrm{~W} @ 50 \Omega$ ). The pre-amplifier consists of a digitally controlled variable-gain amplifier and a monolithic amplifier stage. The power amplifier employs the BLF 188XR LDMOS field effect transistor in a balanced final stage and the MRF6V2010 transistor in a driver stage. The attenuator in the forward path is used to drive the modulator in its linear region, whereas the attenuator and the phase shifter in the feedback path are used to match the RF sampled signal with the reference signal (the input signal) at the comparator input. The phase shifter in the LO path of the demodulator is responsible to realize the proper negative feedback by adjusting the loop phase to $180^{\circ}$, whereas the limiter in the same path is used to feed the LO terminal of the demodulator with a constant signal level independent of the RF input power. The Advanced Design System (ADS simulator) was used to model the entire system where each block in the diagram of Fig. 5.2 is represented to emulate the functionality of the real prototype components. The S-parameters for different coil and phantom configurations have been imported to ADS from EM simulations using FEM 
tool (Ansoft HFSS).

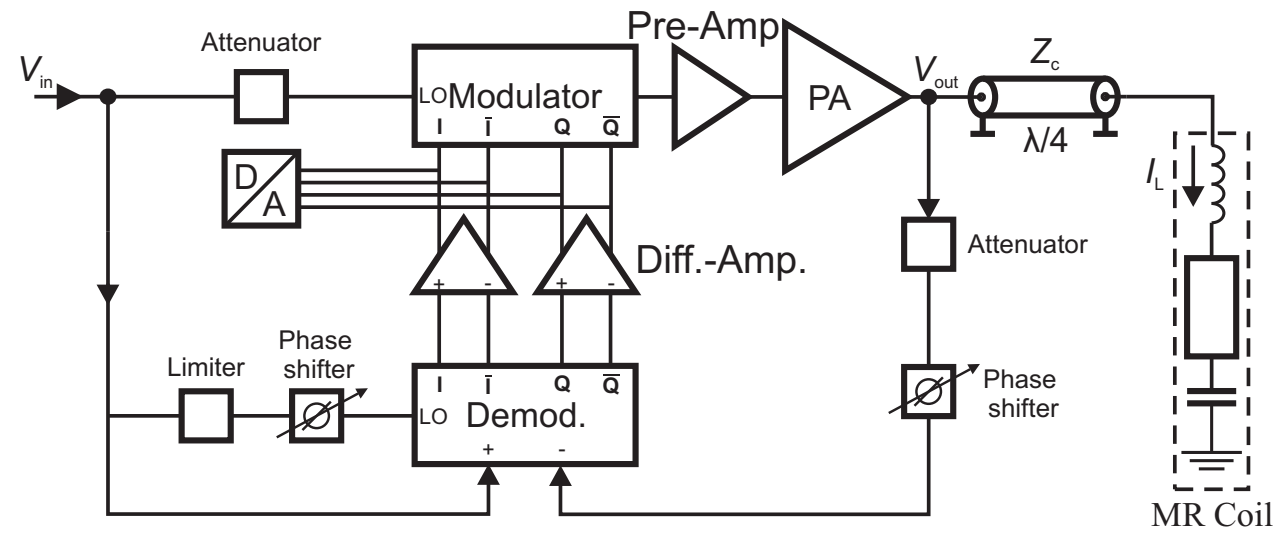

Figure 5.2: Block diagram of simulation setup for one meander coil with unconventional Cartesian Feedback loop power amplifier.

\subsection{Stability Analysis}

Loop stability is considered as a significant challenge faced by feedback loop systems. From loop gain diagram of the open loop transfer function, the stability margin can be obtained. To achieve loop stability, a low-pass filter is used to limit the loop bandwidth. The loop bandwidth should be set high enough to cover all MRI pulses modulation bandwidths required for all MRI applications which may extend up to $100 \mathrm{kHz}$. Three different bandwidth settings are selected to investigate the loop stability while the loop gain is adjusted to the obtainable maximum value before driving the modulator into saturation $(18 \mathrm{~dB})$. Fig. 5.3 shows the loop gain diagram of the open loop transfer function for loop bandwidths $100 \mathrm{kHz}, 500 \mathrm{kHz}$ and $1 \mathrm{MHz}$, while the corresponding stable and unstable regions in the reflection coefficient plane for Pin $=0 \mathrm{dBm}$ are shown in Fig. 5.4. The loop gain frequency response has been obtained by applying the Return Ratio (RR) in section (4.3). From simulation results in Fig 5.4, we conclude that the combination of $18 \mathrm{~dB}$ loop gain with $100 \mathrm{kHz}$ loop bandwidth is the convenient choice to insure loop stability within the region of $-1 \leq \Gamma \leq 1$ in the reflection coefficient plane.

Before we show the improvement in stability that our new feedback design offers through the dynamic loop gain, we prefer to derive the transfer function and the loop gain to get good insight about how the loop works. To ease our task, we will use a simplified version of the block diagram just for analysis which is shown in Fig. 5.5. The transfer function derived in the following ignores the extra forward path (modulator I/Q-bias) which has no influence on the loop stability. 


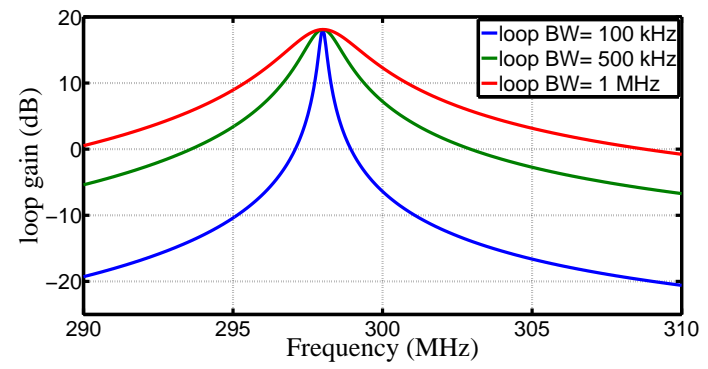

(a)

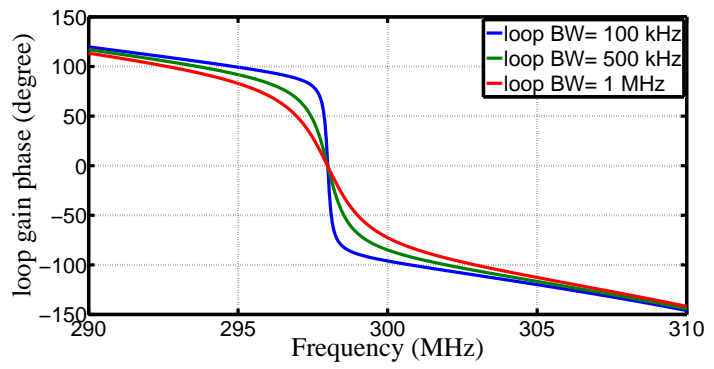

(b)

Figure 5.3: Loop gain diagram of the open loop transfer function for different loop bandwidth. (a) Magnitude, (b) phase.

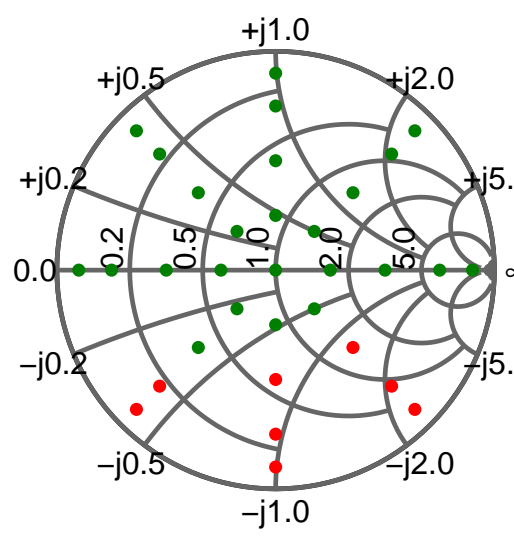

(a)

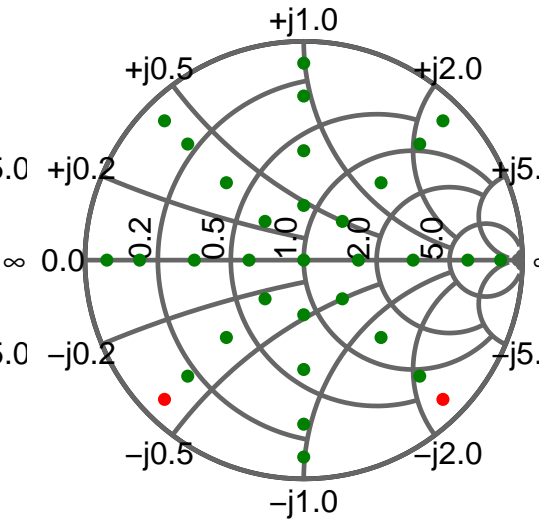

(b)

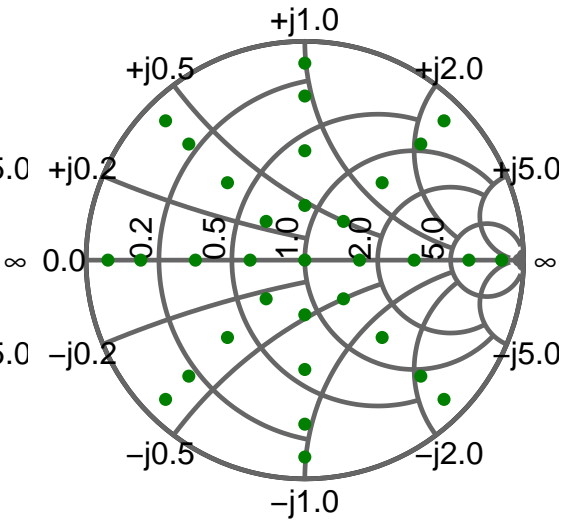

(c)

Figure 5.4: Stable (green color) and unstable (red color) regions for $P i n=0 \mathrm{dBm}$, Pout $=57 \mathrm{dBm}$ and loop bandwidth equal to (a) $1 \mathrm{MHz}$, (b) $500 \mathrm{kHz}$, (c) $100 \mathrm{kHz}$.

The output signal is initially defined by

$$
V_{\text {out }}=V_{\mathrm{m}} A e^{\mathrm{j} \Theta}
$$

The output signal of the modulator can be defined by

$$
V_{\mathrm{m}}=\left(a_{1} V_{\mathrm{in}}\right)\left(V_{\mathrm{e}} k_{\mathrm{d}} F\right) k_{\mathrm{m}} .
$$

$V_{\mathrm{e}}$ as the resulting error signal from the comparison process, defined by

$$
V_{\mathrm{e}}=V_{\text {in }}-a_{2} e^{\mathrm{j} \phi} V_{\text {out }}
$$

The output signal after the substitution of Eq. 5.2 and Eq. 5.3 in Eq. 5.1 is as follows 


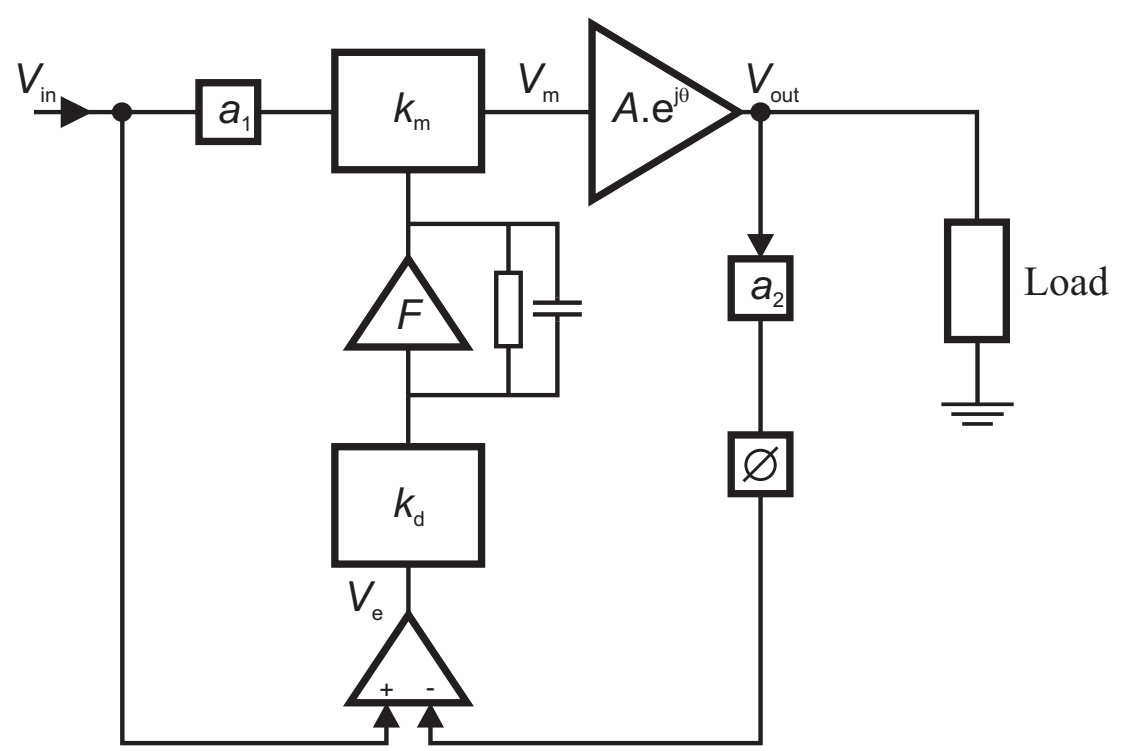

Figure 5.5: Simplified block diagram for unconventional Cartesian feedback loop.

$$
V_{\text {out }}=A e^{\mathrm{j} \Theta}\left(a_{1} V_{\text {in }}\right)\left(k_{\mathrm{d}} F k_{\mathrm{m}}\right)\left(V_{\text {in }}-a_{2} e^{\mathrm{j} \phi} V_{\text {out }}\right)
$$

and after rearrangement we get

$$
V_{\text {out }}=V_{\text {in }}\left[\frac{A e^{\mathrm{j} \Theta} a_{1} V_{\text {in }} k_{\mathrm{d}} F k_{\mathrm{m}}}{1+A e^{\mathrm{j} \Theta} a_{1} V_{\mathrm{in}} k_{\mathrm{d}} F k_{\mathrm{m}} a_{2} e^{\mathrm{j} \phi}}\right] .
$$

Eq. 5.5 shows the closed loop transfer function

$$
G_{\mathrm{CL}}=\left[\frac{A e^{\mathrm{j} \Theta} a_{1} V_{\mathrm{in}} k_{\mathrm{d}} F k_{\mathrm{m}}}{1+\underbrace{A e^{\mathrm{j} \Theta} a_{1} V_{\mathrm{in}} k_{\mathrm{d}} F k_{\mathrm{m}} a_{2} e^{\mathrm{j} \phi}}_{G_{\mathrm{OL}}}}\right],
$$

where $V_{\text {in }}$ is the input signal voltage, $V_{\text {out }}$ is the output signal voltage, $a_{1}$ is the attenuation factor for the input signal, $k_{\mathrm{m}}$ is the modulator gain, $k_{\mathrm{d}}$ is the demodulator gain, $F$ is the transfer function of the active low pass filter, $V_{\mathrm{m}}$ is the input signal voltage of the power amplifier, $A$ is the power amplifier gain, $\Theta$ is the phase shift of power amplifier, $a_{2}$ is the attenuation factor of an attenuator and $\phi$ is the phase shift of a phase shifter in the feedback path. $G_{\mathrm{OL}}$ is the open loop gain. Because an active first order low pass filter is placed in the loop with transfer function

$$
F(\mathrm{j} \omega)=\frac{v}{1+\mathrm{j} \omega \tau}
$$




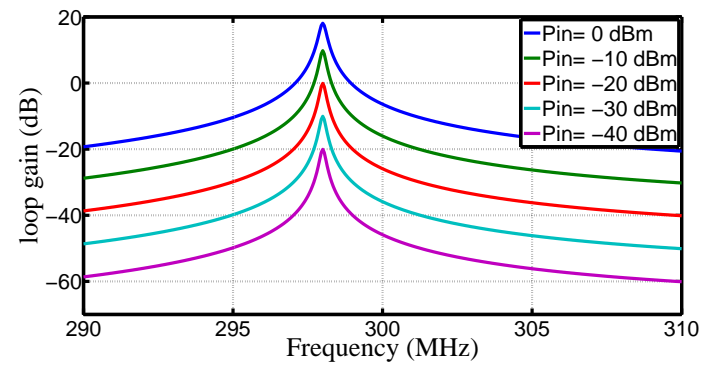

(a)

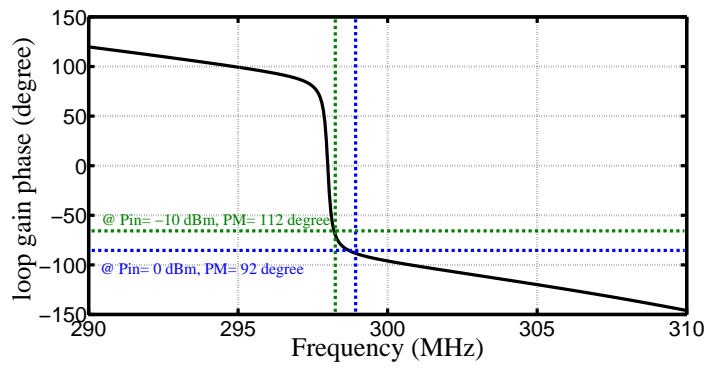

(b)

Figure 5.6: Dynamic loop gain as a function of input power for unconventional Cartesian feedback loop. (a) Magnitude, (b) phase.

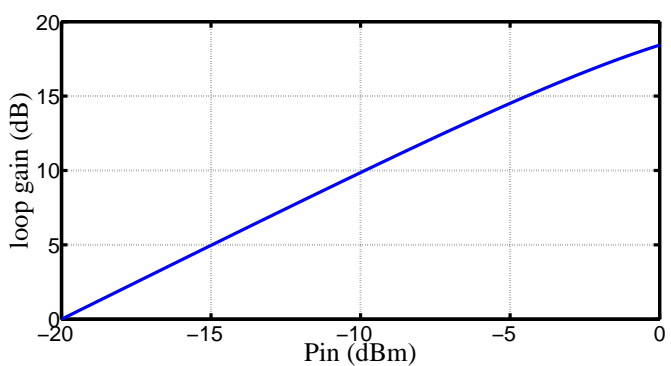

(a)

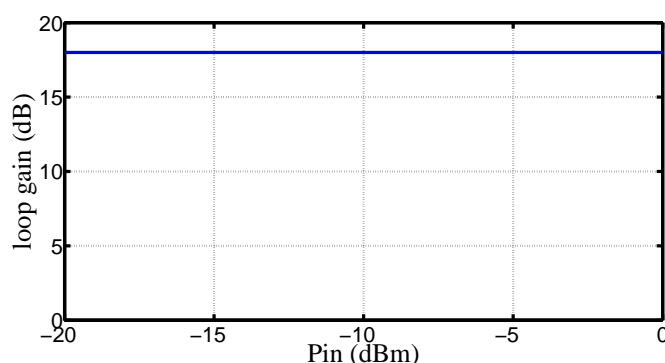

(b)

Figure 5.7: Loop gain as a function of input power for (a) unconventional Cartesian feedback loop, (b) conventional Cartesian feedback loop.

the open loop gain becomes

$$
G_{\mathrm{OL}}=\frac{v A e^{\mathrm{j} \Theta} a_{1} V_{\mathrm{in}} k_{\mathrm{d}} k_{\mathrm{m}} a_{2} e^{\mathrm{j} \phi}}{1+\mathrm{j} \omega \tau}
$$

where $v$ is the gain and $\tau$ is the time constant of the active filter. From Eq. 5.8, it is clear that the open loop gain is proportional to the RF input voltage while its bandwidth is mainly limited by the time constant of the filter. Such effects can be seen explicitly in Fig. 5.6 (a). The dynamically driven modulator creates a dynamic loop gain. This feature increases the stability margin for lower input power as seen in Fig. 5.6 (b): For Pin $=0 \mathrm{dBm}$, the phase margin PM= $92^{\circ}$ and for $\mathrm{Pin}=-10 \mathrm{dBm}, \mathrm{PM}=112^{\circ}$. The proportionality between the loop gain and the input power can be demonstrated in another representative form as seen in Fig. 5.7 (a), whereas in the conventional FBL the loop gain is independent on the input power as seen in Fig. 5.7 (b). This difference in loop gain behaviour between conventional and unconventional feedback loops will show different instability regions in the reflection coefficient plane. In Fig. 5.8, a comparative stability study is presented for both loops when the HPA is terminated with a load characterized 
by high reflection coefficient (i.e., $-2 \leq \Gamma \leq 2$ ). In an array system, high reflection coefficients $|\Gamma|>1$ can be expected due to the coupling to neighbor coils which may be driven much higher than the coil under consideration, e.g., due to RF shimming. For the conventional FBL case (Fig. 5.8(a)), instability regions start to appear directly when the reflection coefficient exceeds one, independent of the excitation power level, while for the unconventional FBL case (Fig. 5.8(b)), an explicit improvement appears in the stability region once the excitation power level of the amplifier decreases.
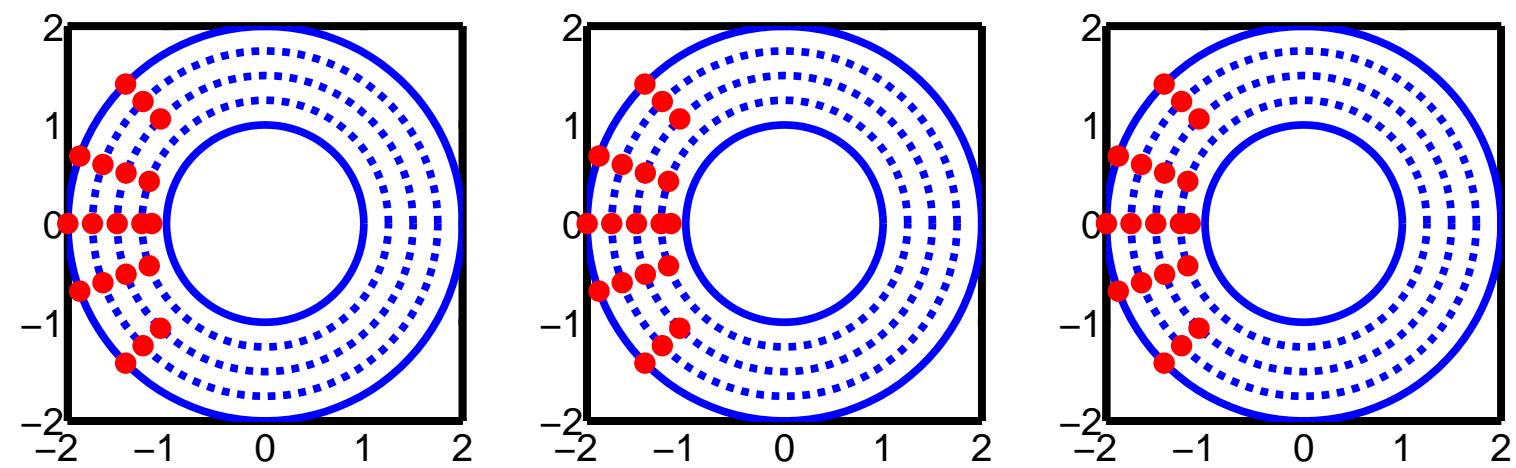

(a)
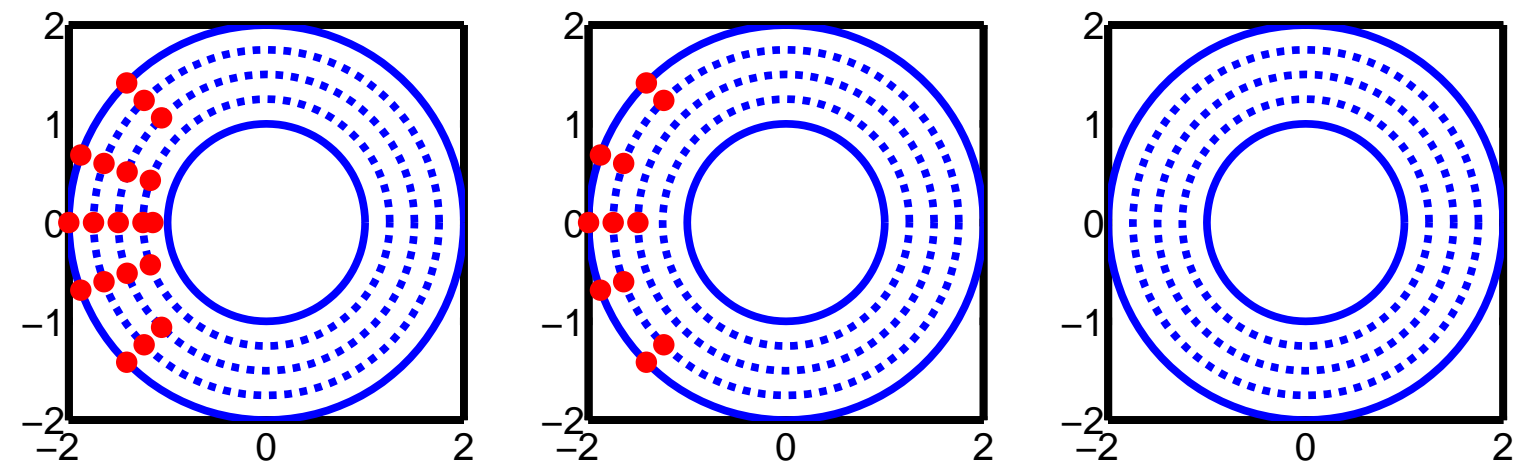

(b)

Figure 5.8: Instability regions in the reflection coefficient plane $(-2 \leq \Gamma \leq 2)$ at $\mathrm{Pin}=0 \mathrm{dBm}$ (left), Pin= $-10 \mathrm{dBm}$ (middle) and Pin= $-20 \mathrm{dBm}$ (right) for (a) conventional Cartesian FBL, (b) unconventional Cartesian FBL.

\subsection{Experimental Prototype}

The fabricated prototype of the control part (combined with the pre-amplifier) of our power amplifier is shown in Fig. 5.9. The input signal with maximum input power of $0 \mathrm{dBm}$ passes through a $25 \mathrm{~dB}$ attenuator before reaching the vector modulator (Analog Devices AD8345) in order to drive it in its linear region. A digital-to-analog converter (DAC) (Maxim MAX5115) 
which is controlled by $\mathrm{I} 2 \mathrm{C}$ bus is used to bias the vector modulator at its quadrature inputs setting the operating point of the amplifier chain and representing the extra forward path. In order to allow an increase of $3 \mathrm{~dB}$ in output power if needed to compensate a mismatch, the bias was set such that the maximum input power would generate $50 \%$ of the saturated power of the PA (3 $\mathrm{dB}$ back-off). The vector modulator output signal drives the digitally controlled variable-gain amplifier (MAX2027) which in turn drives the last stage of the pre-amplifier (Mini Circuits PHA-1+) to end up with a maximum output power of $20 \mathrm{dBm}$. To enable the functionality of the feedback loop, either the I2C bus in combination with an 8-bit register (PCA9554) is used or a manual toggle switch is used. The enable signal controls the logarithmic amplifier (AD8309) and I/Q downconverter (HMC597) by turning them on and off. The logarithmic amplifier is used to provide the local oscillator (LO) signal for the I/Q demodulator with constant signal level independent of the input signal power. The phase adjustment of the LO is achieved by an additional vector modulator controlled by a second DAC. Table 5.1 contains the main components used to build up the Cartesian feedback loop power amplifier with model number, gain and maximum input and output levels. An on-chip RF balun in the "HMC597" downconverter allows differential driving of its RF port which is utilized as a comparator between the RF reference signal (the input signal) and a sample of the RF output signal (to be controlled). In case of a matched load, the probed output voltage signal is adjusted such that the two signals are matched in amplitude and phase so that apart from an off-set voltage no quadrature signals are generated and the amplifier operates as if without feedback loop. Once an error appears due to mismatch or mutual coupling to active neighbouring RF coils, quadrature signals are generated and pass through the active low pass filter (AD8132) with $18 \mathrm{~dB}$ gain and $100 \mathrm{kHz}$ bandwidth before reaching the vector modulator. The vector modulator in turn increases or reduces its gain in the I- and Q-channels in such a way that the error signal is counteracted. This response is limited in particular by the operating point of the amplifier (back-off level) and by the feedback loop gain. In order to verify our simulation model, a loop gain measurement was performed at $-10 \mathrm{dBm}$ input power showing a good agreement with the simulated one, see Fig. 5.10. A test for instability was run by shifting the probed output signal phase around $360^{\circ}$. Oscillations occur as expected with anti-phase at input power levels which yield a loop gain above $0 \mathrm{~dB}$. 


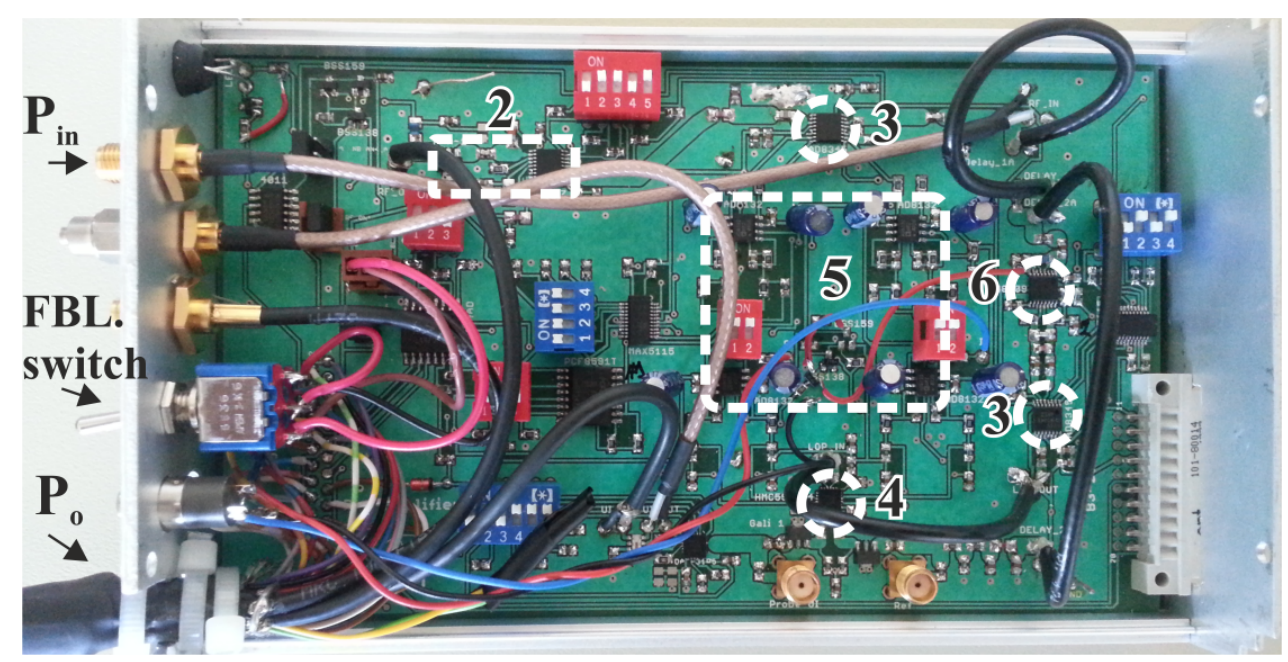

Figure 5.9: The prototype of our unconventional Cartesian feedback loop control unit including the pre-amplifier.

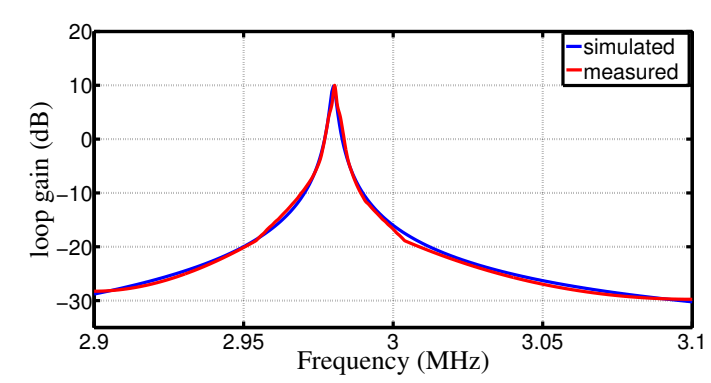

(a)

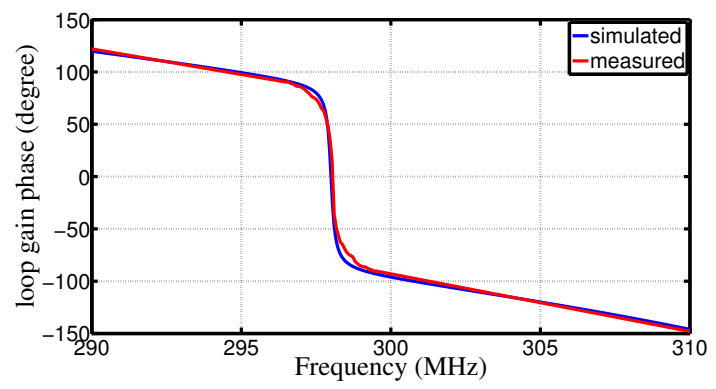

(b)

Figure 5.10: Measured and simulated loop gain. (a) Magnitude, (b) phase.

Table 5.1: Components of power amplifier and feedback loop.

\begin{tabular}{|c|c|c|c|c|}
\hline Component & Model number & $\begin{array}{c}\text { Gain } \\
(\mathrm{dB})\end{array}$ & $\begin{array}{c}\text { Max Input } \\
\left(\mathrm{dB}_{\mathrm{m}}\right)\end{array}$ & $\begin{array}{c}\text { Max Output } \\
\left(\mathrm{dB}_{\mathrm{m}}\right)\end{array}$ \\
\hline 1. High Power Amplifier & $\begin{array}{c}\text { MRF6V2010 } \\
\text { +BLF188XR }\end{array}$ & 46 & +20 & +62 \\
\hline 2. Pre-Amplifier & $\begin{array}{c}\text { Max2027 } \\
\text { PHA-1+ }\end{array}$ & 8 to 31 & +20 & +20 \\
\hline $\begin{array}{c}\text { 3. Up- } \\
\text { Converter/Modulator }\end{array}$ & AD8345 & up to +25 & +10 & +2 \\
\hline $\begin{array}{c}\text { 4. Down- } \\
\text { Converter/Demodulator }\end{array}$ & HMC597 & -6 & +20 & +7 \\
\hline $\begin{array}{c}\text { 5. Active Low pass } \\
\text { filter }\end{array}$ & AD8132 & +16 & - & - \\
\hline $\begin{array}{c}\text { 6.Logarithmic } \\
\text { Amplifier/Limiter }\end{array}$ & AD8309 & +80 to -30 & +22 & -10 \\
\hline
\end{tabular}




\subsection{Performance Evaluation of Feedback Loop}

\subsubsection{Loading Effect}

In 2.4.1, the effect of loading on the coil impedance as well as on the resonance frequency of the coil has been demonstrated. This will also effect the output voltage of the power amplifier. In our simulation, the coil has been tuned to the Larmor frequency for 7T (298 MHz) and matched to $50 \Omega$ under loading condition when the phantom is placed $200 \mathrm{~mm}$ above the coil. This means, the reference output voltage is equal to $224 \mathrm{~V}$ at $d=200 \mathrm{~mm}$, when the power amplifier is driven into $3 \mathrm{~dB}$ back-off from saturation $(500 \mathrm{~W})$. Fig. 5.11 shows the output voltages by sweeping the phantom distance $d$ above the coil with and without FBL activated for two different matching scenarios as used in Fig. 2.12. It is distinguishable from the figure that the residual error produced by matching the coil at the Larmor frequency is larger than that produced by matching the coil at resonance. The main objective of using our Cartesian feedback technique is to compensate for the mismatch of the loaded RF coils at the Larmor frequency as good as possible. Because the coil current is proportional to the power amplifier output voltage based on the current forcing property of a quarter wave transmission line (section 3.6.2), we need to maintain the output voltage and phase at the same level as for the matched load case. The feedback loop gain and the back-off level of the amplifier chain are the key parameters responsible for the limitation of the compensation for amplifier load mismatch, seen in Fig. 5.11 (c)(d). Rise time delay is noticeable for the PA with feedback loop activated in comparison with the case where no feedback loop is active. This delay appears due to the limited bandwidth of the loop which is dominated by the low pass filter. A demonstration of output voltage $V_{\text {out }}$ due to coil loading can also be shown in the complex domain as seen in Fig. 5.12. For both matching scenarios, we see the complex voltage as function of distance $d$ when the FBL is off or activated to compensate the error.

What we can conclude from Fig. 5.11 and Fig. 5.12 is that the output voltage error becomes considerable once the phantom comes very close to the coil and the FBL performance shows its limitation to correct such a significant error. To evaluate the performance of our feedback loop, the residual error was analysed. The residual error definition that we have followed is given by:

$$
e_{\mathrm{R}}=\left|x_{\mathrm{r}}-x_{\mathrm{a}}\right|
$$

where $e_{\mathrm{R}}$ is the residual error, $x_{\mathrm{r}}$ is the reference value and $x_{\mathrm{a}}$ is the actual value. The reference value is considered to be the value obtained by connecting a matched load. After calculating the 


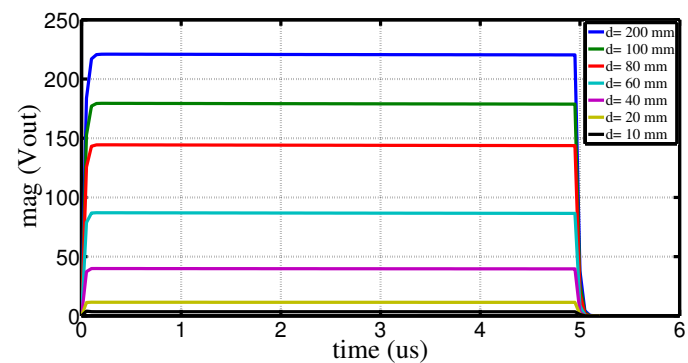

(a)

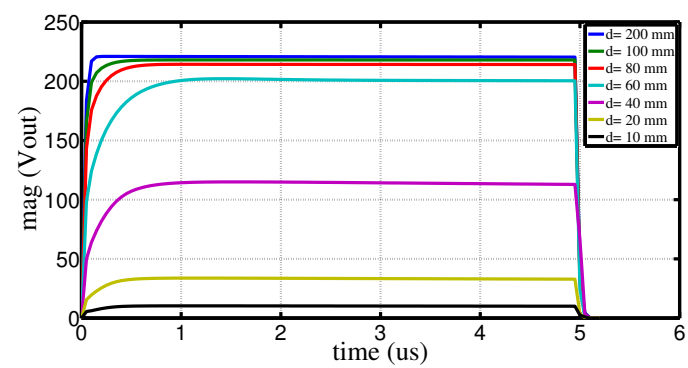

(c)

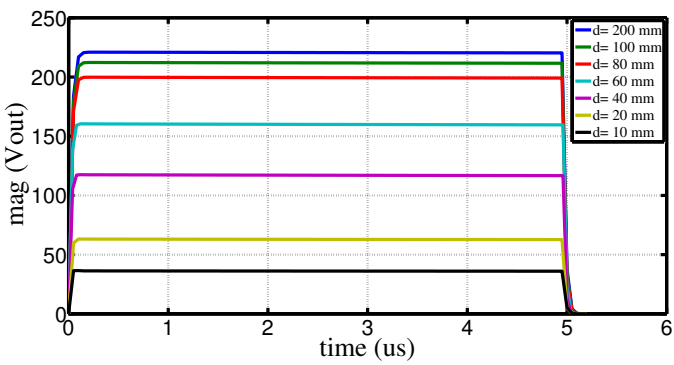

(b)

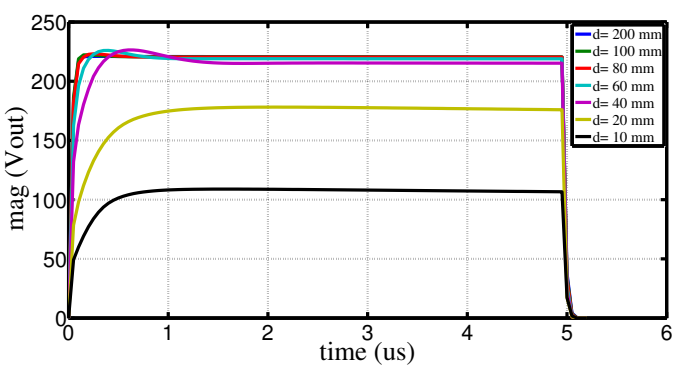

(d)

Figure 5.11: Variation of power amplifier output voltage $V_{\text {out }}$ by sweeping phantom distance $d$. (a) When the coil is matched at Larmor frequency without FBL, (b) when the coil is matched at each new resonant frequency without FBL, (c) when the coil is matched at Larmor frequency with FBL, (d) when the coil is matched at each new resonant frequency with FBL.

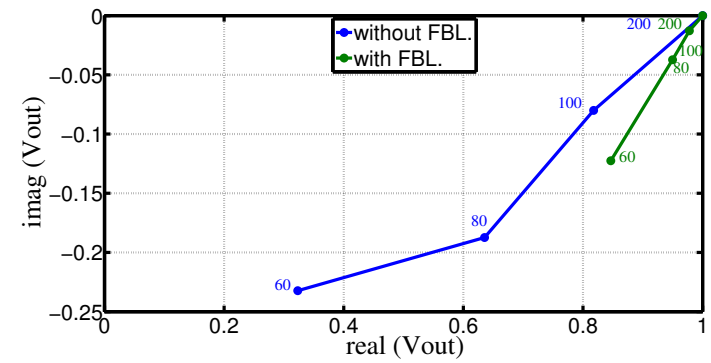

(a)

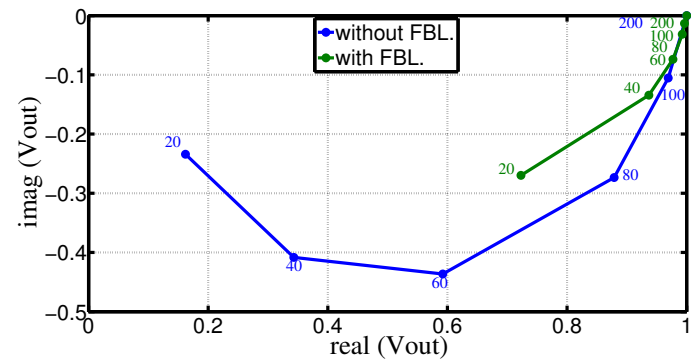

(b)

Figure 5.12: Normalized output voltage in complex domain for different coil loading situations. (a) When the coil is matched at Larmor frequency, (b) when the coil is matched at each new resonance frequency.

residual errors for each case, the mean residual error is calculated for both magnitude and phase which is given by:

$$
\overline{e_{\mathrm{R}}}=\frac{\sum e_{\mathrm{R}}}{N},
$$

where $\overline{e_{\mathrm{R}}}$ is the mean residual error, $\sum e_{\mathrm{R}}$ is the summation of all errors and $N$ is the number of cases. To get a meaningful value of the mean residual error for the magnitude, a percentage representation is desirable with respect to the reference value which is given by: 


$$
E_{\mathrm{R}} \%=\frac{\overline{e_{\mathrm{R}}}}{x_{\mathrm{r}}} \times 100
$$

where $E_{\mathrm{R}}$ is the percentage representation of mean residual error with respect to the reference value. Table 5.2 summarizes $E_{\mathrm{R}}$ for magnitude and $\overline{e_{\mathrm{R}}}$ for phase. These results have been calculated for all loading situations used in Fig. 5.11.

Table 5.2: Error $E_{\mathrm{R}}$ for output voltage magnitude and phase with and without feedback loop.

\begin{tabular}{|c|c|c|c|c|}
\hline \multirow{2}{*}{$E_{\mathrm{R}}, \overline{e_{\mathrm{R}}}$} & \multicolumn{2}{|c|}{ Magnitude } & \multicolumn{2}{c|}{ Phase } \\
\cline { 2 - 5 } & Matching at Larmor & Matching at resonance & Matching at Larmor & Matching at resonance \\
\hline Without FBL & $64.84 \%$ & $40.42 \%$ & $40.23^{\circ}$ & $39.83^{\circ}$ \\
\hline With FBL & $41.44 \%$ & $13.70 \%$ & $28.00^{\circ}$ & $11.79^{\circ}$ \\
\hline
\end{tabular}

\subsubsection{Coupling Effect}

In 2.4.2, the effect of the mutual coupling between the coil array elements on the coil impedance has been demonstrated. The change in the impedance will change the delivered voltage to the coil (i.e. the output voltage of the power amplifier). In this subsection we try to evaluate the performance of our Cartesian feedback to compensate for the mutual coupling effect by calculating the residual error for magnitude and phase of the output voltage. To do this, we have driven the first element in Fig. 2.15(a) using our PA with the Cartesian Feedback shown in Fig. 5.2 with $P_{\text {in }}=0 \mathrm{dBm}$ to deliver a power of $57 \mathrm{dBm}$ to the coil. Simultaneously, the second element in Fig. 2.15(a) is driven by a signal power $52 \mathrm{dBm} \leq P_{\mathrm{G} 2} \leq 72 \mathrm{dBm}$ and generator phase between $0^{\circ}$ and $180^{\circ}$. The coupling coefficient between the two elements was approximately $-18 \mathrm{~dB}$. A demonstration of output voltage $V_{\text {out }}$ in Fig. 5.2 which corresponds to $V_{1}$ in Fig. 2.15(a) due to mutual coupling can be shown in the complex domain with and without FBL activated for two different generator phases: Fig. 5.13(a) shows the first case of excitation $\left(\angle V_{\mathrm{G} 2}=0^{\circ}\right)$ while the signal power is swept. It is obvious that the output signal voltages is larger than the reference output voltage (blue line). Fig. 5.13(b) shows the second case of excitation $\left(\angle V_{\mathrm{G} 2}=\right.$ $180^{\circ}$ ) where the output signal voltages behaves inversely in comparison to the first case. Table 5.3 summarizes the evaluation of FBL performance for both cases which shows a significant improvement in residual error.

In both cases the activated FBL reduces the deviations in the terminal voltage and by this stabilizes the coil current against induced current by the neighbor coil. 


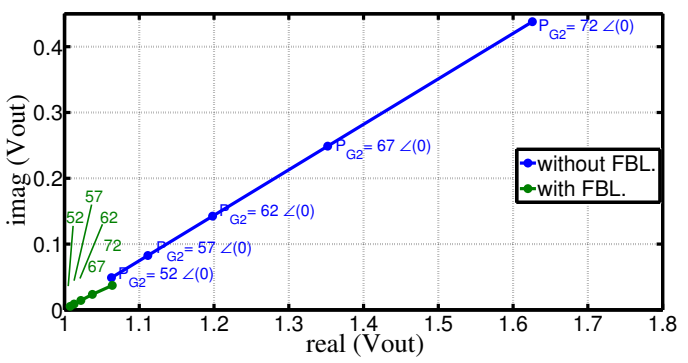

(a)

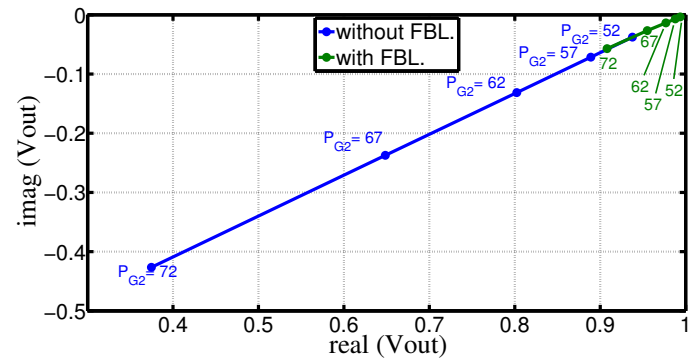

(b)

Figure 5.13: Normalized output voltage in the complex domain for different coupling power in $\mathrm{dBm}$ when the phase of the second generator is set to (a) $0^{\circ}$, (b) $180^{\circ}$.

Table 5.3: Error $E_{\mathrm{R}}$ for output voltage magnitude and phase due coupling effect with and without feedback loop.

\begin{tabular}{|c|c|c|c|c|}
\hline \multirow{2}{*}{$E_{\mathrm{R}}, \overline{e_{\mathrm{R}}}$} & \multicolumn{2}{|c|}{ Magnitude } & \multicolumn{2}{c|}{ Phase } \\
\cline { 2 - 5 } & $@ \Theta=0^{\circ}$ & $@ \Theta=180^{\circ}$ & $@ \Theta=0^{\circ}$ & $@ \Theta=180^{\circ}$ \\
\hline Without FBL & $28.87 \%$ & $22.00 \%$ & $7.47^{\circ}$ & $17.36^{\circ}$ \\
\hline With FBL & $2.78 \%$ & $3.50 \%$ & $0.98^{\circ}$ & $1.28^{\circ}$ \\
\hline
\end{tabular}

\subsubsection{Linearization}

High power amplifiers are preferred to operate as close to saturation as possible in order to achieve high power efficiency. However, by driving the PA around its compression point, the output signal will be distorted nonlinearly. In other words, the amplitude deviates from a proportional (straight line) response and the phase deviates from a constant response creating so-called amplitude modulation-to-amplitude modulation (AM-AM) distortion and amplitude modulation-to-phase modulation (AM-PM) distortion as discussed in chapter 3. This distortion is connected to a deviation of the amplifier transfer characteristic from the linear characteristic. Various power amplifier linearization techniques were developed to overcome the variation in the behaviour of a power amplifier. This section will discuss our Cartesian feedback technique used to linearize the Class AB high power amplifier. The first step is to characterize the distortion of the amplifier without feed back loop by plotting the transfer characteristic of magnitude, phase and gain, and then observe the difference when the feedback loop is activated. From Fig. 5.14, it is obvious that Class $\mathrm{AB}$ behaves nonlinearly within a certain region even before it reaches the saturation level. The main objective of the Cartesian feedback is to keep the output magnitude aligned with the linear response characteristic, the output phase aligned with the input signal phase and keep the gain constant. The linearization performance of our Cartesian feedback is noticeable in Fig. 5.14 where we find good improvement up to the PA full saturation level. 


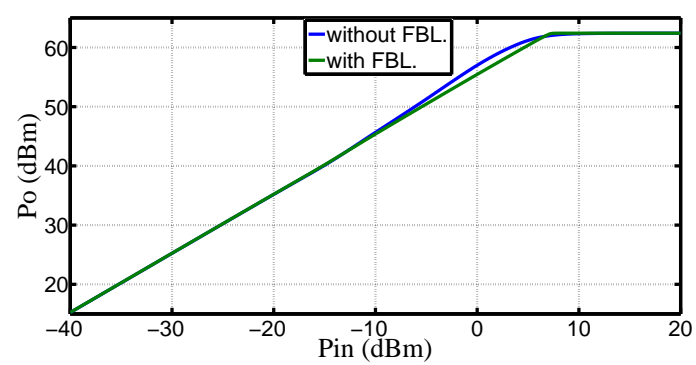

(a)

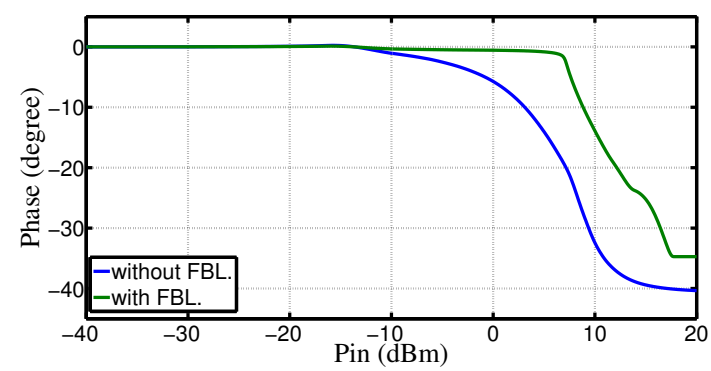

(b)

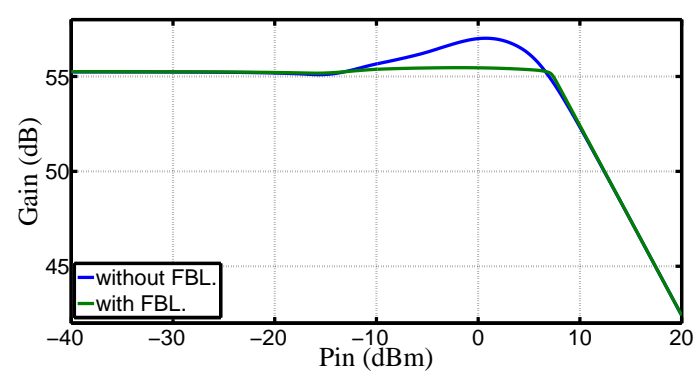

(c)

Figure 5.14: Power amplifier transfer characteristic with and without feedback loop. (a) Magnitude in $\mathrm{dBm}$, (b) phase, (c) gain.

\subsubsection{FBL Effect on Output Impedance}

In section 5.3, we have demonstrated how our FBL improves the stability when the exciting input power decreases. This improvement, on the other hand, decreases the loop gain. Which in turn decreases the performance of the FBL in terms of error compensation. This section will show the advantage of combining the FBL with an ultra-low output impedance PA (ULOI PA) with respect to the delivered output voltage. If we start at an input power equal to $0 \mathrm{dBm}$, which drives the PA into $3 \mathrm{~dB}$ back-off, we will get maximum loop gain. Thus, the FBL error correction performance will be at its maximum. In this case, the FBL performs better than the ULOI PA as seen in Fig. 5.15(a). This advantage decreases when the exciting input power is 
reduced as seen in Fig. 5.15(b-d). When the PA is driven by lower input power $(<-30 \mathrm{dBm})$, the ULOI PA will take-over most of the error compensation. However, we also recognize that the ability of the ULOI PA to operate as a voltage source (keeping the output voltage at a fixed level) improves as the drive power is reduced. From this combination of ULOI PA and FBL we conclude the following: At high drive level the error compensation by the FBL improves the ultra-low impedance properties of the PA in the area of low load impedances. With low drive level, the FBL becomes stable over a wider range of (active) load reflection coefficients but at the same time loses its effect on the stabilization of the output voltage while the output impedance properties of the PA improves and provides a good stabilization of the output voltage.

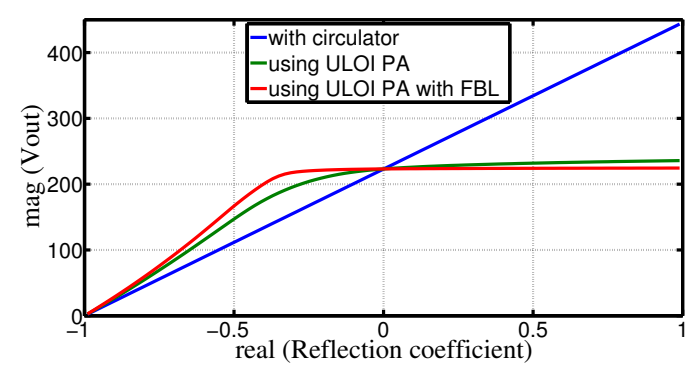

(a)

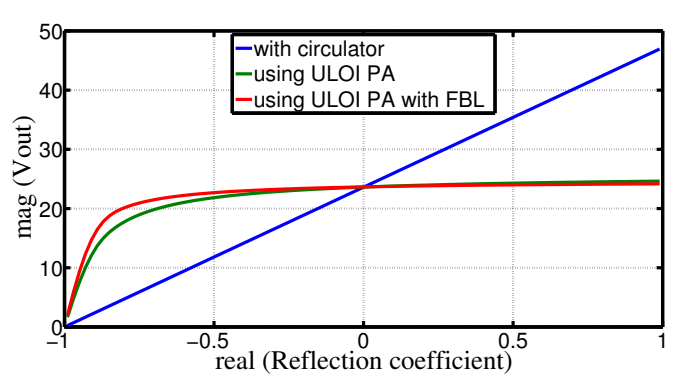

(c)

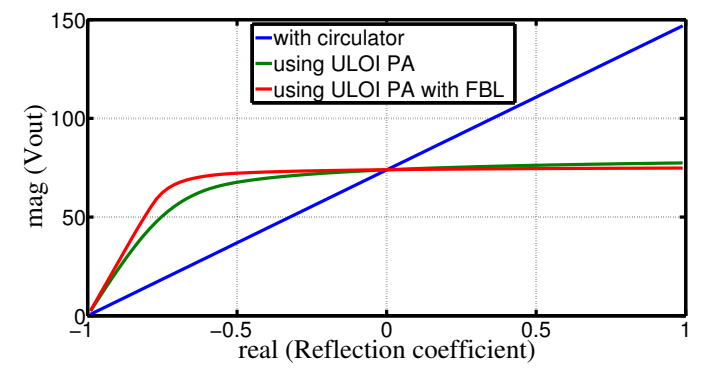

(b)

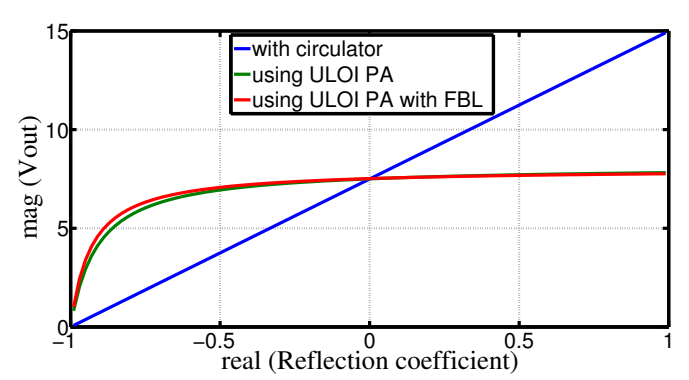

(d)

Figure 5.15: Comparison of PA output voltage using the ULOI PA and using the ULOI PA in combination with the FBL at (a) Pin $=0 \mathrm{dBm}$, (b) Pin $=-10 \mathrm{dBm}$, (c) Pin= $-20 \mathrm{dBm}$, (d) Pin= $30 \mathrm{dBm}$. 



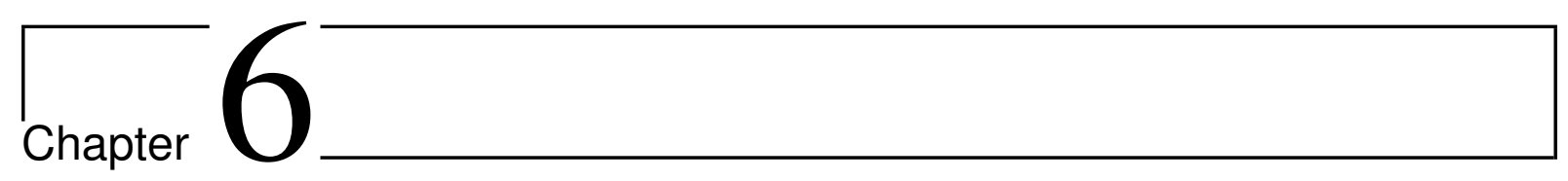

\section{CONCLUSION}

A new combination of near-magnet PA with MR coil has been successfully used to demonstrate the remote sensing of current (and voltage) at the coil. This new concept of current sensing is considered valuable in a coil array setup where multi-channels are required to be fitted within a small area (pTx system). In earlier realizations, current sensors were integrated in a transmit coil where each sensor establishes its own channel, and for a large number of array elements, this method becomes hard to apply.

The critical component in our new system is the cable between the PA and the coil. Its effective electrical length has to be precisely met at the operating frequency. In addition, the cable attenuation not only reduces the power delivered to the coil but also degrades the measurement accuracy for the coil current. An acceptable measurement accuracy is obtained by a low-loss cable with maximum length of about $4 \mathrm{~m}$. In case of a near-magnet PA, this length is quite sufficient to bridge the distance to the coil at the center of the magnet bore.

In our PA, the unconventional Cartesian feedback is used to stabilize the output voltage and by this to control the current in the coil. The feedback loop gain and the back-off level of the amplifier chain are the key parameters limiting the compensation performance. With its maximum loop gain occurring at high input power levels, our FBL compensates efficiently for current variation due to coil mismatch and coupling to neighbor coils. In addition, it linearises the Class AB power amplifier.

Due to its particular feedback loop gain dependence on the input signal power level, the error compensation at high input power level relies on the FBL, whereas at low input power level, the error compensation relies on the low output impedance of the PA.

In a pTx-system, high reflection coefficients larger than 1 can be expected due to induction 
from neighbor coils, in particular when coils are driven with large difference in power level due to, e.g., RF shimming. Therefor, a PA driven at low power level requires higher immunity against instability than a PA at high drive level and this is what the unconventional Cartesian FBL provides.

While the mentioned characteristics of our PA allows its use near the MR magnet, a comparison to the conventional solution of PA combined with a circulator highlights a practical disadvantage: Due to the direct connection of the load without isolation by a circulator, the gain, impedance, compression level and maximum output power are more or less dependent on the load impedance. Although this can be either calibrated out or taken into account in the calculation of array excitation (solving nonlinear equations), pTx operation requires much higher lead effort than with a conventional transmitter system. 


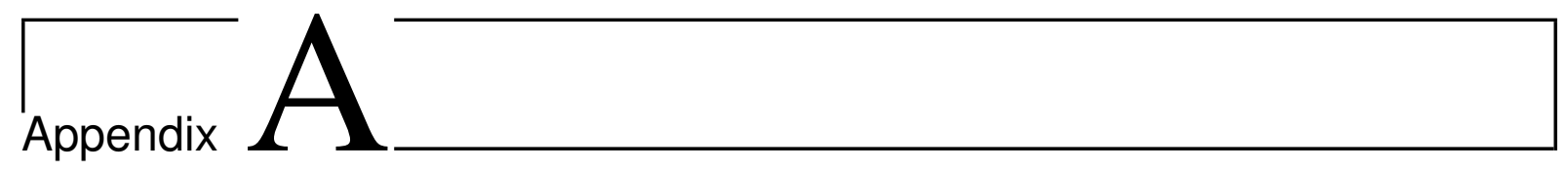

\section{Appendix A: PA Prototype}

The following figures show the boards of the high power part of the power amplifier without the shielding cassette.

The PA circuit employs a PCB based on Rogers RO4003 laminate bonded to a ground plane which is made out of a machined aluminum heatsink. The power transistors are screwed down to

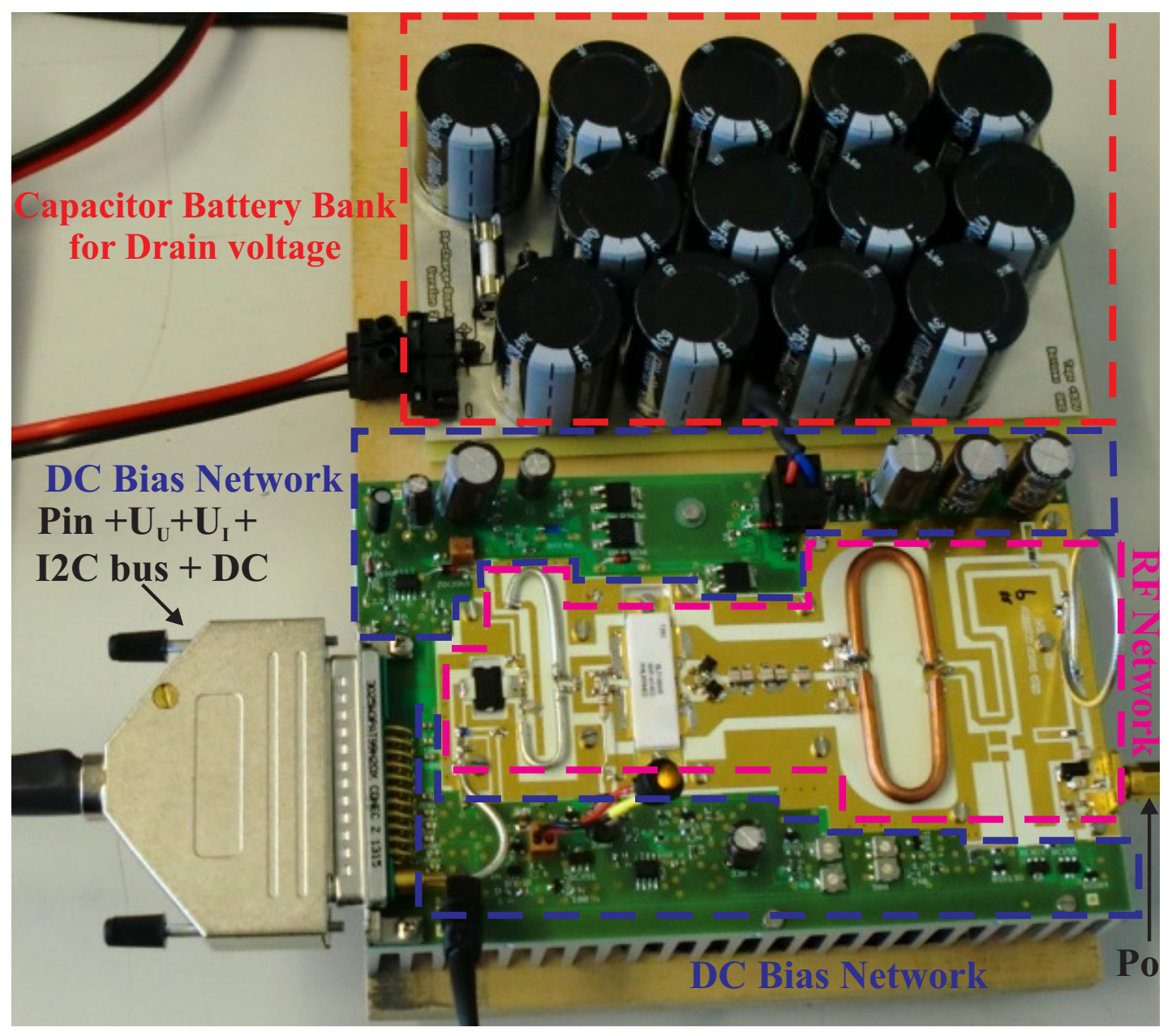

Figure A.1: PA board with capacitor bank. 


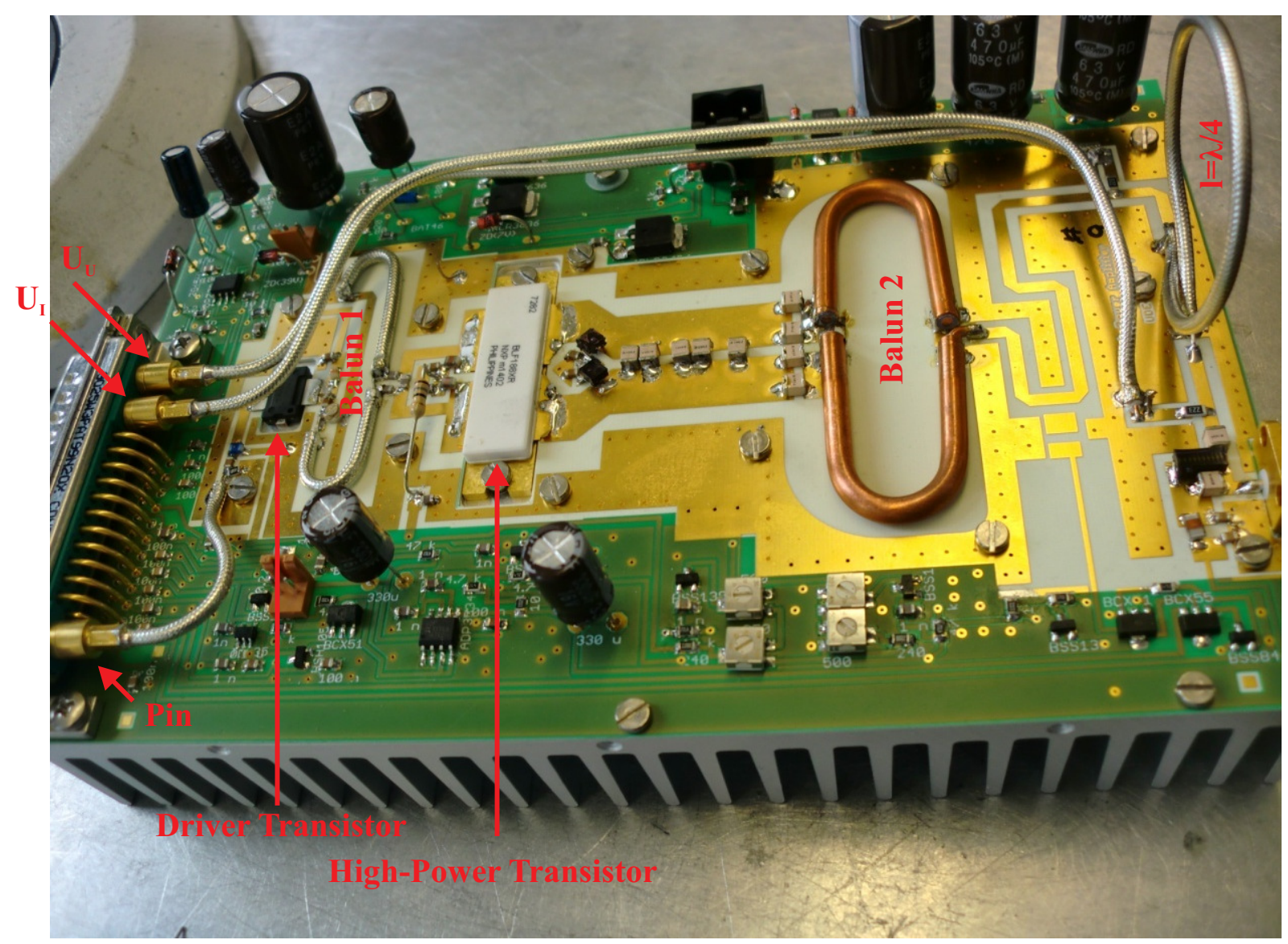

Figure A.2: High power amplifier board with the voltage probes $\left(U_{\mathrm{I}}\right.$ and $\left.U_{\mathrm{U}}\right)$ and $\lambda / 4$ transmission line in the output section.

the heatsink through openings in the PCB for better heat transfer. At both sides of the RF circuit, analog circuits control the Gate bias and stabilize the Drain voltage, among other functions. Since the power amplifier is used in pulse mode only, the Drain voltage supply is based on a PCB combining a large number of electrolytic capacitors (giving a total of $5 \mathrm{mF}$ ) charged up to $53 \mathrm{~V}$ by a remote power supply. The bank of capacitors sustains a peak pulse current of $30 \mathrm{~A}$ over a maximum pulse length of $5 \mathrm{~ms}$ with a voltage drop of 1.t. $4 \mathrm{~V}$ and a series regulator circuit close to the RF power transistors on the RF board keeps the Drain voltage constant at $48 \mathrm{~V}$. 


\section{Appendix B: PA Characteristics}

The following figures represent some experimental results for the fabricated power amplifier. Note that these are not directly applicable as verification of simulation results in chapter 4-5, since the fabricated PAs include several design modifications and are operated under conditions mostly deviating from those in the simulations.

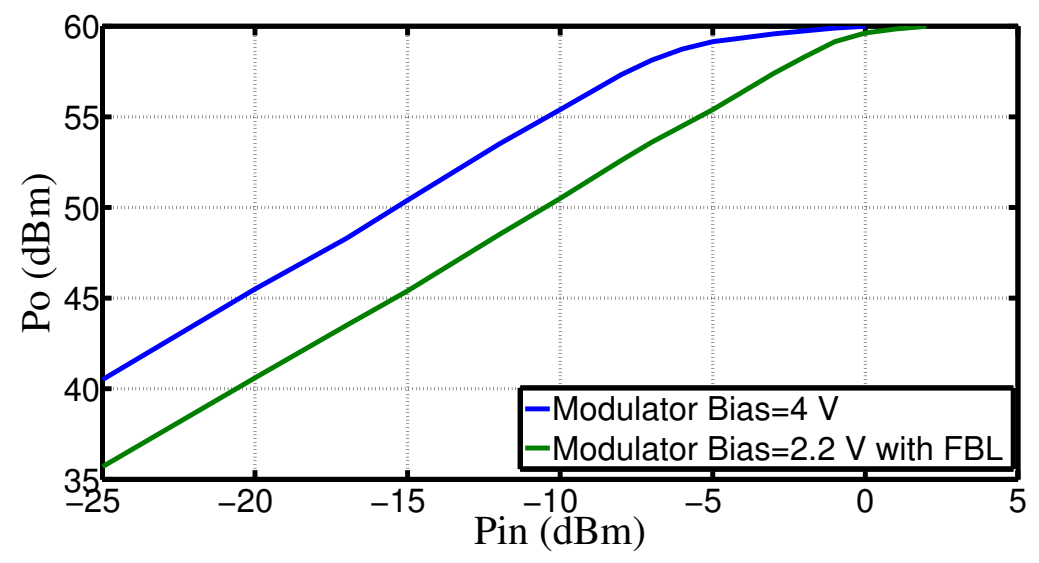

Figure B.1: Measured PA transfer characteristic.

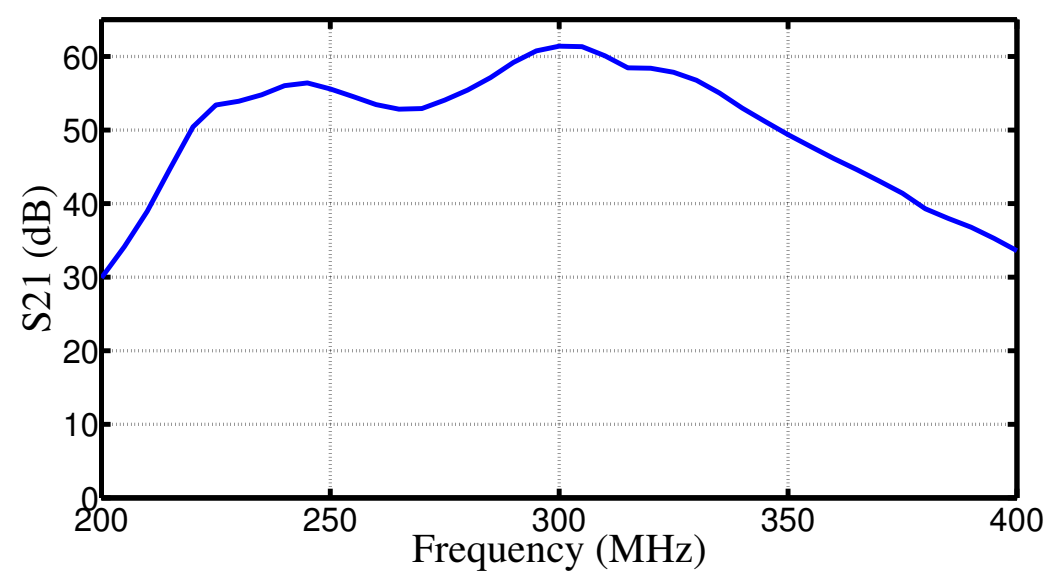

Figure B.2: Measured PA frequency response for a drive level in the linear region. 


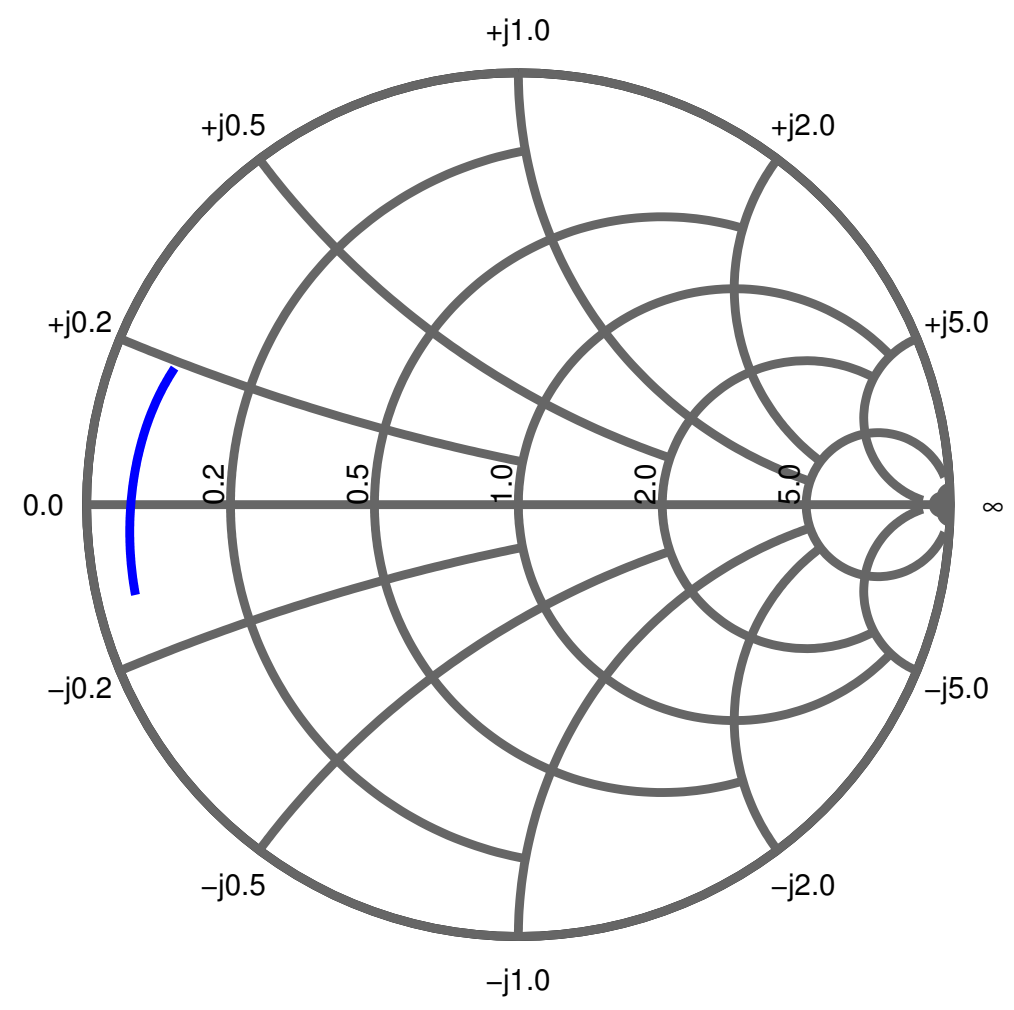

Figure B.3: Measured PA output impedance as a function of frequency between $290 \mathrm{MHz}$ and $310 \mathrm{MHz}$ (PA is deactivated but powered).

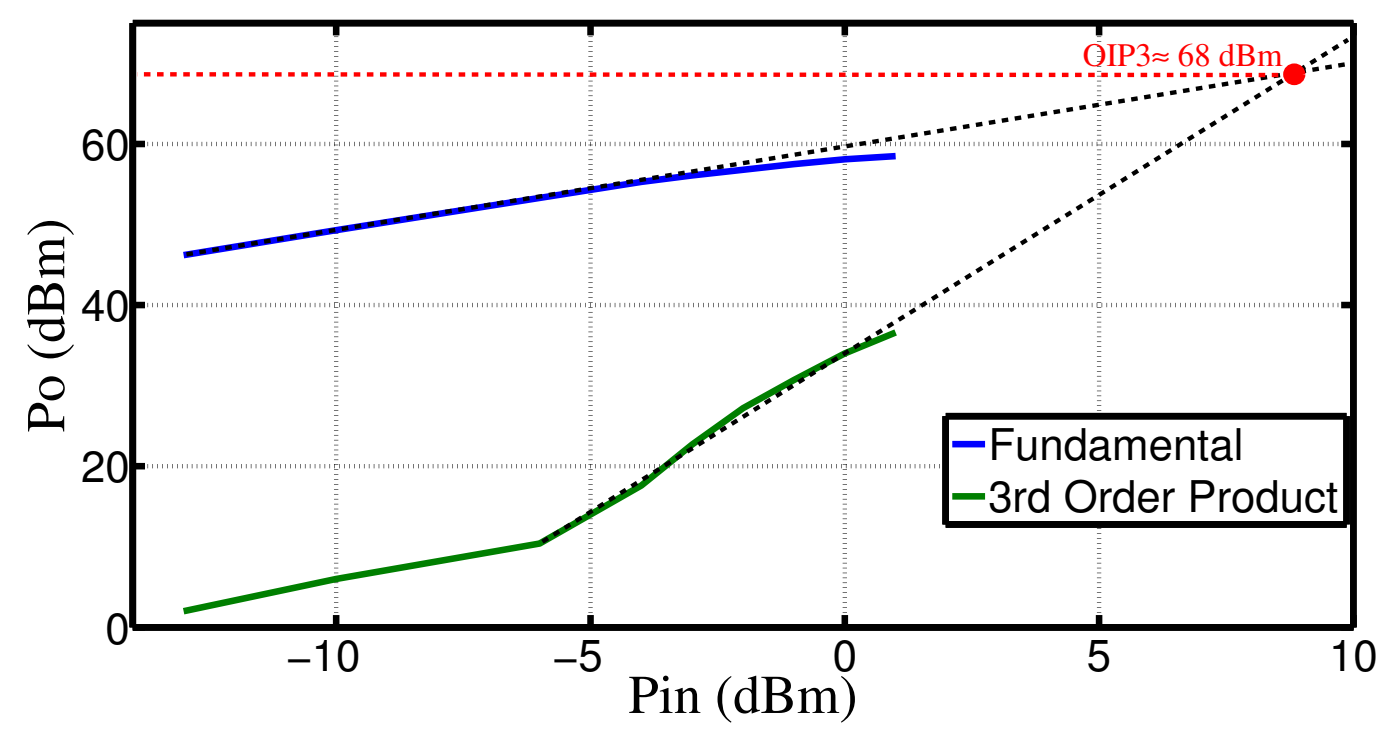

Figure B.4: Measured third-order intercept due to intermodulation distortion of two test signals $200 \mathrm{kHz}$ apart. 


Appendix

\section{Appendix C: Experimental investigation of}

\section{coil-PA interaction}

The following figures present results of an experiment to verify the interaction of the PA and the coil when the coil impedance is varied by moving close to a phantom. The coil feed current is measured by a magnetic current probe and is compared to the $U_{\mathrm{I}}$-probe voltage.

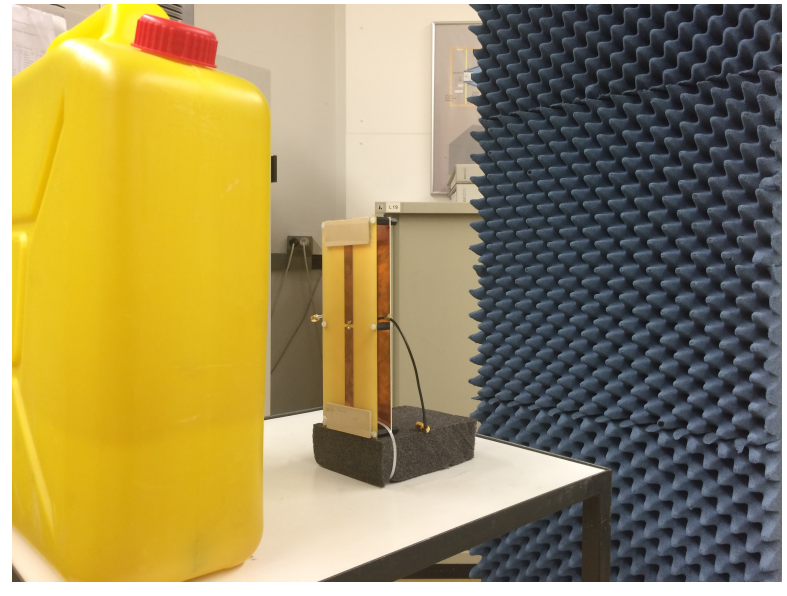

(a)

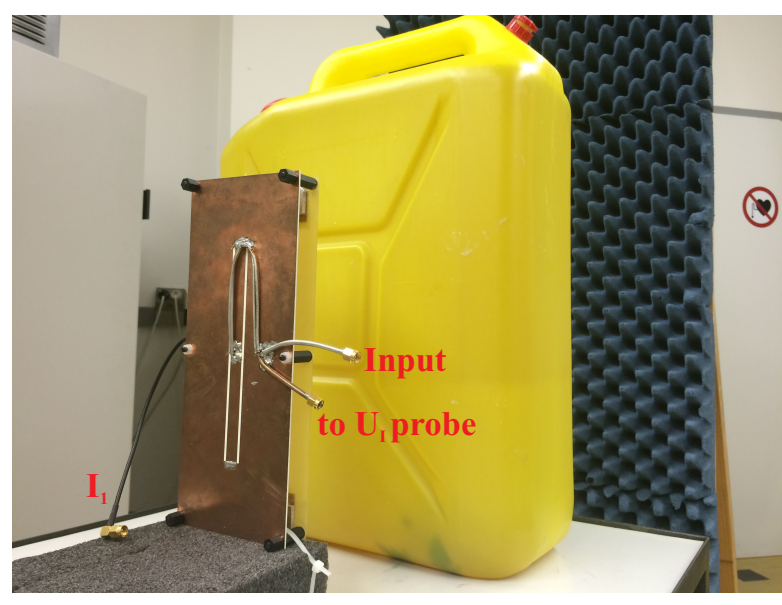

(b)

Figure C.1: Measurement setup for coil reflection coefficient and coil current measurements. (a) Meander dipole coil approaches the phantom in steps, (b) meander coil seen from the back with balun and cable connections to the input and a voltage probe at the balun input (representing $U_{\mathrm{I}}$ ). 


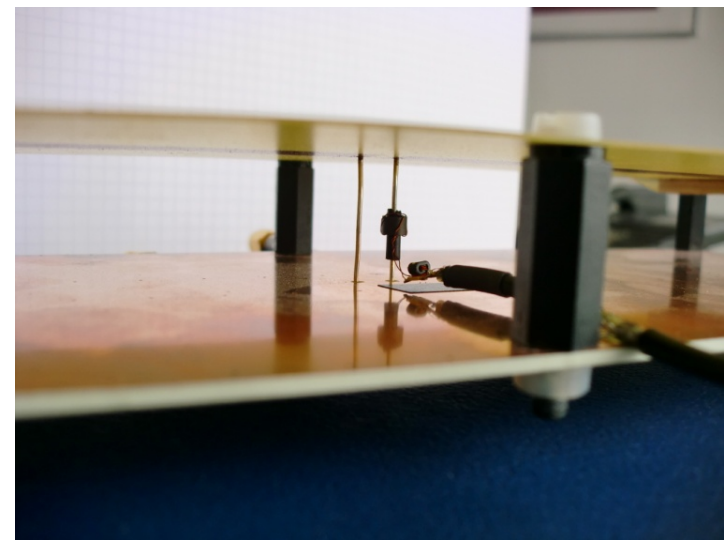

Figure C.2: Magnetic current probe at meander dipole feed point.

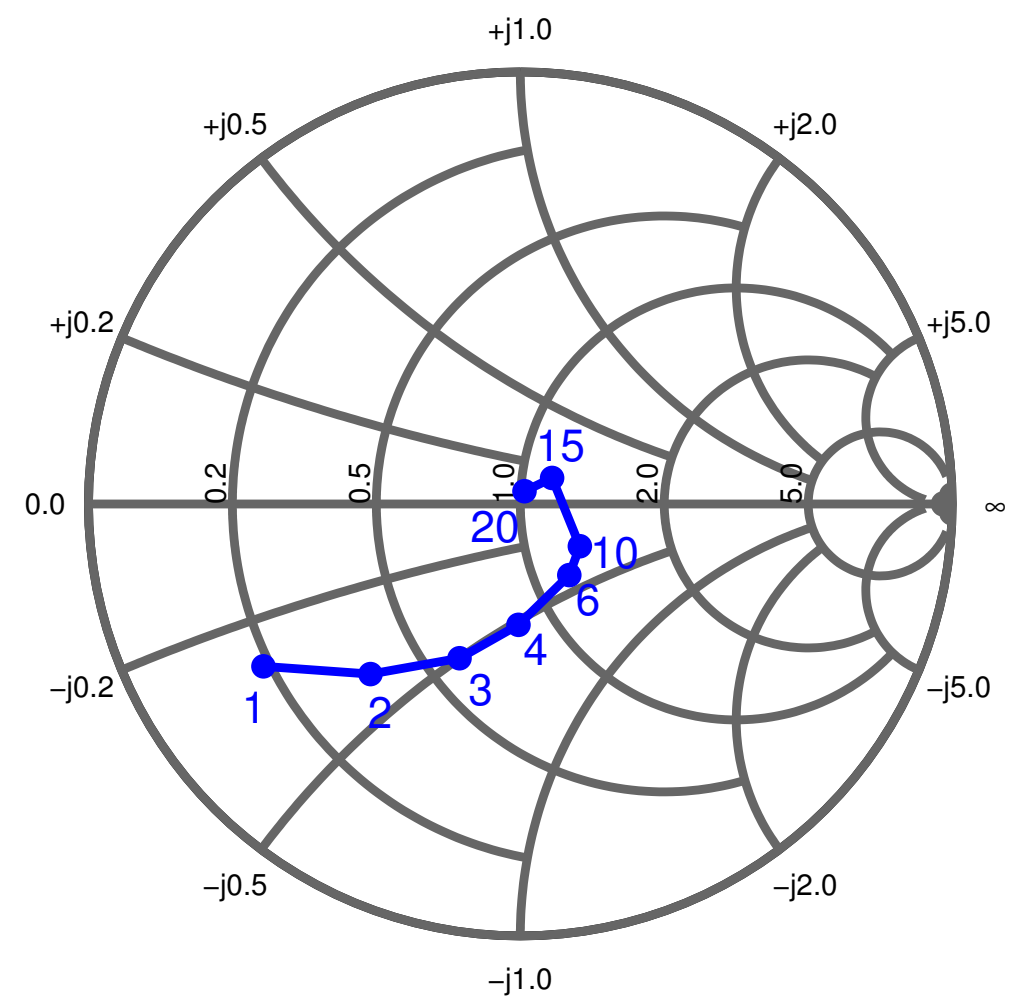

Figure C.3: Demonstration of coil reflection coefficient variation due to coil loading with phantom distance varying from $1 \mathrm{~cm}$ to $20 \mathrm{~cm}$. 


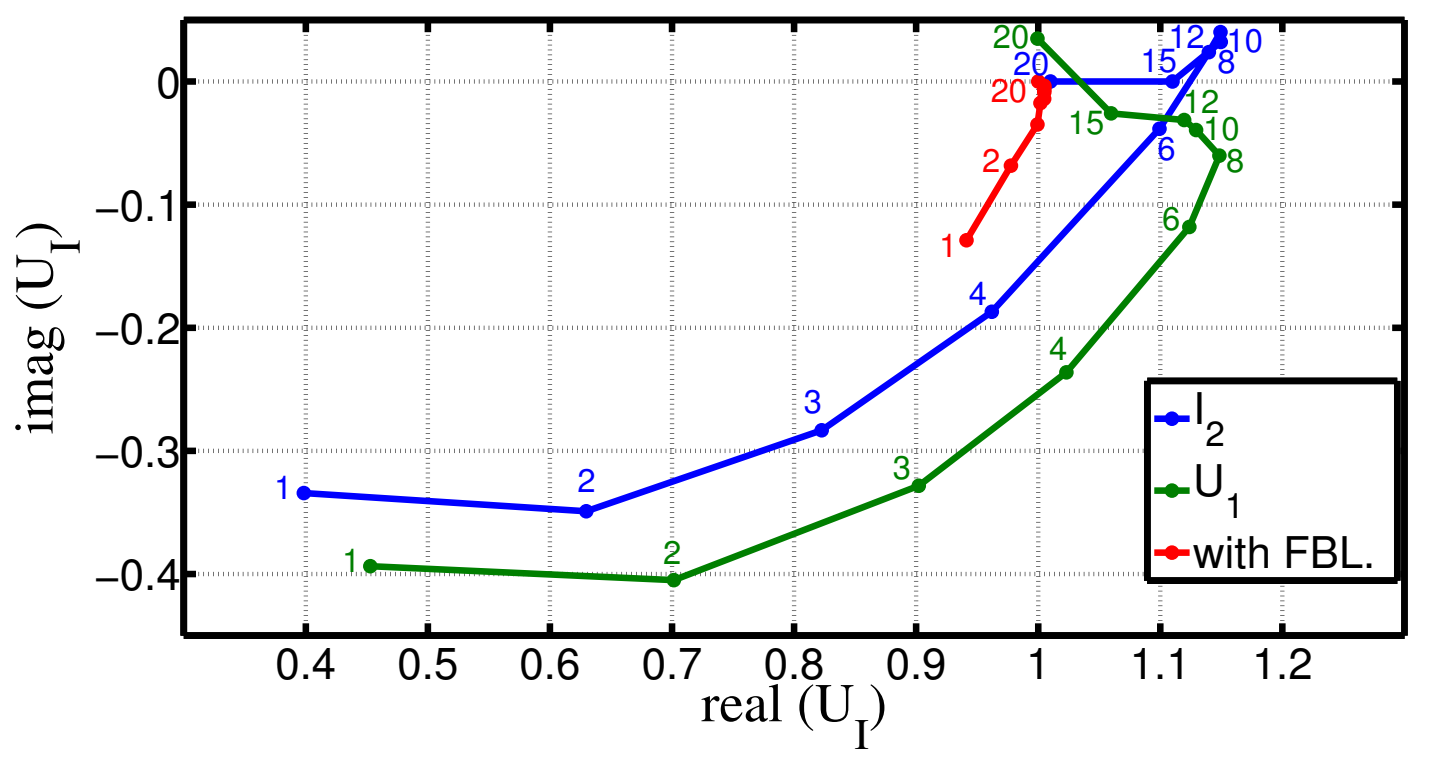

Figure C.4: Comparison of coil current variation due to coil loading using the magnetic current probe (blue) and the probe voltage $U_{\mathrm{I}}$ at the PA-Output (green). In red: $U_{\mathrm{I}}$ with correction due to FBL. 



\section{LIST OF PUBLICATIONS}

1. Abuelhaija, A; Solbach, K: "Parasitic Element Based Decoupling of 7 Tesla MRI", LAPC 2015, Loughborough, November 2015.

2. Abuelhaija, A; Solbach, K: "An Ultra-Law Output Impedance Power Amplifier for Tx Array in 7-Tesla Magnetic Resonance Imaging", ICMST 2015, Barcelona, August 2015.

3. Abuelhaija, A; Solbach, K: "7T Coil Decoupling in Near-Magnet Power Amplifier", ISMRM 2015, Toronto, May 2015.

4. Orzada, S; Bitz, A; Gratz, M; Johst, S; Völker, M; Kraff, O; Beyer, D; Mathiebe, T; Abuelhaija, A; Solbach, K: "An Integrated 8-Channel Tx/Rx Body Coil for 7 Tesla Whole-Body MRI", ISMRM 2015, Toronto, May 2015.

5. Abuelhaija, A; Solbach, K; Orzada, S: "Comprehensive Study on a Coupled Meandered Microstrip line RF Coil Elements for 7-Tesla Magnetic Resonance Imaging", EuCAP 2015, Lisbon, April 2015.

6. Abuelhaija, A; Solbach, K; Buck, A: "Power Amplifier for Magnetic Resonance Imaging using Unconventional Cartesian Feedback Loop", GeMiC 2015, Nürnberg, March 2015.

7. Solbach, K; Abuelhaija, A; Shooshtary, S: "Near-Magnet Power Amplifier with built-in Coil Current Sensing", ISMRM 2014, Milan, May 2014. 



\section{BIBLIOGRAPHY}

[1] Z. Mahmood, B. Guerin, B. Keil, E. Adalsteinsson, L. Wald, and L. Daniel, "Design of a robust decoupling matrix for high field parallel transmit arrays," in Proceedings of the 22nd Annual Meeting and Exhibition of ISMRM, Milan, Italy, 2014.

[2] P. Roemer, W. Edelstein, C. Hayes, S. Souza, and O. Mueller, “The NMR phased array,” Magnetic resonance in medicine, vol. 16, no. 2, pp. 192-225, 1990.

[3] J. Jevtic, "Ladder networks for capacitive decoupling in phased-array coils," in Proceedings of the 9th Annual Meeting of ISMRM, Glasgow, Scotland, vol. 17, 2001.

[4] B. Wu, X. Zhang, P. Qu, and G. X. Shen, "Design of an inductively decoupled microstrip array at 9.4 T," Journal of Magnetic Resonance, vol. 182, no. 1, pp. 126-132, 2006.

[5] A. Abuelhaija, K. Solbach, and S. Orzada, "Parasitic Element Based Decoupling of 7 Tesla MRI Coil Array,” LAPC 2015, Loughborough, UK, November 2015.

[6] Y. Soutome, Y. Otake, and Y. Bito, "Vertical loop decoupling method for gapped phasedarray coils," in Proc. Intl. Soc. Mag. Reson. Med, vol. 19, 2011, p. 1859.

[7] W. Lee, E. Boskamp, T. Grist, and K. Kurpad, "Radiofrequency current source (RFCS) drive and decoupling technique for parallel transmit arrays using a high-power metal oxide semiconductor field-effect transistor (MOSFET)," Magnetic Resonance in Medicine, vol. 62, no. 1, pp. 218-228, 2009.

[8] X. Chu, Y. Liu, J. Sabate, and Y. Zhu, "Ultra-low output impedance RF power amplifier array," in Proc. Intl. Soc. Mag. Reson. Med, vol. 15, 2007, p. 172. 
[9] A. Abuelhaija and K. Solbach, "An Ultra-Low Output Impedance Power Amplifier for Tx Array in 7-Tesla Magnetic Resonance Imagine," in International Conference on Microwave Science and Technology, Barcelona, Spain, vol. 2, no. 8, 2015, p. 1422.

[10] D. Hoult, G. Kolansky, D. Kripiakevich, and S. King, “The NMR multi-transmit phased array: a Cartesian feedback approach,” Journal of Magnetic Resonance, vol. 171, no. 1, pp. 64-70, 2004.

[11] D. Hoult, G. Kolansky, and D. Kripiakevich, "A 'Hi-Fi' Cartesian feedback spectrometer for precise quantitation and superior performance," Journal of Magnetic Resonance, vol. 171, no. 1, pp. 57-63, 2004.

[12] M. G. Zanchi, “Cartesian Feedback Control for MRI Transmitter Array Systems,” Ph.D. dissertation, 2010.

[13] D. Hoult, D. Foreman, G. Kolansky, and D. Kripiakevich, "Overcoming high-field RF problems with non-magnetic Cartesian feedback transceivers," Magnetic Resonance Materials in Physics, Biology and Medicine, vol. 21, no. 1-2, pp. 15-29, 2008.

[14] I. Grattan-Guinness, A survey of his life and work based on a critical edition of his monograph on the propagation of heat presented to the Institut de France in 1807. Cambridge (Mass.) ; London : MIT press, 1972.

[15] A. Roguin, "Nikola Tesla: The man behind the magnetic field unit," Journal of magnetic resonance imaging, vol. 19, no. 3, pp. 369-374, 2004.

[16] N. Tubridy and C. McKinstry, "Neuroradiological history: Sir Joseph Larmor and the basis of MRI physics," Neuroradiology, vol. 42, no. 11, pp. 852-855, 2000.

[17] M. S. Rabi I, Zacharias J and K. P., "A new method of measuring nuclear magnetic moments," Phys. Rev. 53:318, 1938.

[18] F. Bloch, W. Hanson, and P. M., “Nuclear Infraction,” Phys. Rev. 69:127, 1946.

[19] E. Purcell, H. Torrey, and R. Pound, "Resonance absorption by nuclear magnetic moments in a solid," Phys. Rev. 69:37-38, 1946.

[20] E. L. Hahn, “Spin echoes,” Phys. Rev., vol. 80, pp. 580-594, Nov 1950. 
[21] R. Damadian, “Tumor detection by nuclear magnetic resonance," Science, vol. 171, no. 3976, pp. 1151-1153, 1971.

[22] C. H and Y. I, "Britain's brains produce first NMR scans," New Scientist, 80: 588, 1978.

[23] R. Hawkes, G. Holland, W. Moore, E. Roebuck, and B. Worthington, "Nuclear magnetic resonance (NMR) tomography of the normal heart." Journal of computer assisted tomography, vol. 5, no. 5, pp. 605-612, 1981.

[24] P. Sprawls, Magnetic resonance imaging: principles, methods, and techniques. Medical Physics Publishing, 2000.

[25] R. D. Watkins, R. H. Caverly, and W. E. Doherty, "298 MHz Micro miniature 2KW Transmit Receive Switch for 7.0 Tesla TR Arrays," in $20^{\text {th }}$ Proc. Intl. Soc. MRM, p. 2686, 2012.

[26] P. Harvey, "High Field Imaging - An Overview of Technical Challenges," Weekend Syllabus, MR Physics For Physicists - Day 2, Overview of Technical Challenges, Proc. ISMRM 2006.

[27] V. de Moortele, C. Akgun, G. Adriany, S. Moeller, J. Ritter, C. M. Collins, M. B. Smith, J. T. Vaughan, and K. Ugurbil, "B1 destructive interferences and spatial phase patterns at $7 \mathrm{~T}$ with a head transceiver array coil," Magnetic resonance in medicine, vol. 54, no. 6, pp. 1503-1518, 2005.

[28] J. T. Vaughan, C. J. Snyder, L. J. DelaBarre, P. J. Bolan, J. Tian, L. Bolinger, G. Adriany, P. Andersen, J. Strupp, and K. Ugurbil, "Whole-body imaging at 7T: Preliminary results," Magnetic resonance in Medicine, vol. 61, no. 1, pp. 244-248, 2009.

[29] M. A. Bernstein, "Field Strength Dependence in MRI: Advantages and Artifacts at 3T," in Proceedings of the ISMRM, 2006.

[30] C. M. Collins, W. Liu, B. J. Swift, and M. B. Smith, "Combination of optimized transmit arrays and some receive array reconstruction methods can yield homogeneous images at very high frequencies," Magnetic resonance in medicine, vol. 54, no. 6, pp. 1327-1332, 2005. 
[31] G. J. Metzger, C. Snyder, C. Akgun, T. Vaughan, K. Ugurbil, V. de Moortele et al., "Local B1+ shimming for prostate imaging with transceiver arrays at 7T based on subjectdependent transmit phase measurements," Magnetic Resonance in Medicine, vol. 59, no. 2 , pp. 396-409, 2008.

[32] U. Katscher, P. Börnert, C. Leussler, and J. S. Van Den Brink, “Transmit SENSE,” Magnetic Resonance in Medicine, vol. 49, no. 1, pp. 144-150, 2003.

[33] S. Orzada, S. Maderwald, B. A. Poser, A. K. Bitz, H. H. Quick, and M. E. Ladd, "RF excitation using time interleaved acquisition of modes (TIAMO) to address B1 inhomogeneity in high-field MRI," Magnetic Resonance in Medicine, vol. 64, no. 2, pp. 327-333, 2010.

[34] D. Brunner, N. Zanche, J. Froehlich, D. Baumann, and K. Pruessmann, "A symmetrically fed microstrip coil array for 7T," in Proc. 15th Annu. Meeting ISMRM, 2007, p. 448.

[35] S. Sohn, A. Gopinath, and J. T. Vaughan, "Electrically auto-tuned RF coil design," International Society for Magnetic Resonance in Medicine (ISMRM), Montreal, Canada, May, vol. 7, p. 13, 2011.

[36] S. Orzada, A. Bahr, and T. Bolz, "A novel 7 T microstrip element using meanders to enhance decoupling," Meander, vol. 1, no. 36, pp. 10-7, 2008.

[37] Z. Chen, K. Solbach, D. Erni, and A. Rennings, "Dipole RF element for 7 Tesla magnetic resonance imaging with minimized SAR," in Antennas and Propagation (EuCAP), 2013 $7^{\text {th }}$ European Conference on. IEEE, 2013, pp. 1775-1778.

[38] G. Saleh, K. Solbach, A. Rennings, and Z. Chen, "SAR Reduction for Dipole RF Coil Element at 7 Tesla by using Dielectric Overlay," in Loughborough Antennas \& Propagation Conference (LAPC 2012), pp. 12-13.

[39] S. Orzada, K. Solbach, M. E. Ladd, and A. K. Bitz, "Comparison of three different microstrip transmit elements for use in multichannel Tx/Rx body coils at 7 Tesla," in $22^{\text {nd }}$ Proc. Intl. Soc. MRM, p. 1308, 2014.

[40] A. Abuelhaija, K. Solbach, and S. Orzada, "Comprehensive study on coupled meandered microstrip line rf coil elements for 7-Tesla magnetic resonance imaging," EUCAP(2015), Lisbon, Portugal, April 2015.

[41] C. A. Balanis, Antenna theory: analysis and design. John Wiley \& Sons, 2005, vol. 1. 
[42] U. Bulus, C. Famdie, and K. Solbach, "Equivalent-circuit modelling of chassis radiator," in German Microwave Conference, 2009. IEEE, 2009, pp. 1-4.

[43] J. T. Vaughan and J. R. Griffiths, RF coils for MRI. John Wiley \& Sons, 2012.

[44] A. M. S. Ahmed, "Analysis, Modelling and Linearization of Nonlinearity and Memory Effects in Power Amplifiers used for Microwave and Mobile Communications," Ph.D. dissertation, Universitaet Kassel, 2005.

[45] G. C. Scott, “MRI transmitter amplifier systems," proc. Intl. Soc. Mag. Reson. Med. 21 (2013).

[46] D. Chattopadhyay, Electronics (fundamentals and applications). New Age International, 2006.

[47] Basic Radio \& Television, 2/E. McGraw-Hill Education (India) Pvt Limited, 2003. [Online]. Available: https://books.google.de/books?id=-vSUwc8UMRwC

[48] J. Shumaker, R. Basset, and A. Skuratov, "High-power GaAs FET amplifiers: Push-pull versus balanced configurations," APPLIED MICROWAVE AND WIRELESS, vol. 14, no. 5, pp. 26-33, 2002.

[49] M. K. Kazimierczuk, RF power amplifier. John Wiley \& Sons, 2014.

[50] P. B. Kenington, High linearity RF amplifier design. Artech House, Inc., 2000.

[51] L. Rade and B. Westergren, Mathematics handbook for science and engineering. Springer Science \& Business Media, 2013.

[52] J. L. Walker, Handbook of RF and microwave power amplifiers. Cambridge University Press, 2011.

[53] K. Solbach, A. Abuelhaija, and S. Shooshtary, "Near-magnet power amplifier with built-in coil current sensing," in 22 $2^{\text {nd }}$ Proc. Intl. Soc. MRM, p. 1287, 2014.

[54] M. Parsamoghadam, S. Shooshtary, and K. Solbach, "Investigation of a Voltage Probe for Cartesian Feedback Power Amplifier Used in 7T Parallel Transmit MRI,' ESMRMB, Edinburgh, UK, October 2015. 
[55] F. M. Ghannouchi and M. S. Hashmi, Load-pull techniques with applications to power amplifier design. Springer Science \& Business Media, 2012, vol. 32.

[56] F. Ghannouchi and M. Hashmi, "Load-pull techniques and their applications in power amplifiers design,” in 2011 IEEE Bipolar/BiCMOS Circuits and Technology Meeting, 2011.

[57] S. Nawaz and H. Mehmood, "Stabilization of microwave amplifiers," 9th International Multitopic Conference, IEEE INMIC 2005.

[58] J. Collantes, N. Otegi, A. Anakabe, N. Ayllon, A. Mallet, and G. Soubercaze-Pun, "Montecarlo stability analysis of microwave amplifiers," in Wireless and Microwave Technology Conference (WAMICON), 2011 IEEE 12th Annual. IEEE, 2011, pp. 1-6.

[59] A. Suárez, S. Jeon, and D. Rutledge, "Stability analysis and stabilization of power amplifiers," Microwave Magazine, IEEE, vol. 7, no. 5, pp. 51-65, 2006.

[60] J. Jugo, J. Portilla, A. Anakabe, A. Suárez, and J. Collantes, “Closed-loop stability analysis of microwave amplifiers," Electronics Letters, vol. 37, no. 4, pp. 226-228, 2001.

[61] S. A. Maas, Nonlinear microwave and RF circuits. Artech House, 2003.

[62] F. Wang, "Design and analysis of high-efficiency L-band power amplifiers," Ph.D. dissertation, California Institute of Technology, 2006.

[63] F. Wang, A. Suárez, and D. B. Rutledge, "Bifurcation analysis of stabilization circuits in an L-band LDMOS 60-W power amplifier," Microwave and Wireless Components Letters, IEEE, vol. 15, no. 10, pp. 712-714, 2005.

[64] K. M. Gilbert and R. S. Menon, "Construction of Receive Arrays: A Four-Channel Rat Coil for 9.4 T,” proc. Intl. Soc. Mag. Reson. Med. 23 (2015).

[65] A. Blumlein and H. Clark, "Amplifier using negative feedback loop," U.K. Patent 425 553, September 1933.

[66] H. S. Black, "Stabilized Feedback Amplifiers," Bell System Technical Journal, vol. 13, no. 1, pp. 1-18, 1934.

[67] H. Nyquist, "Regeneration theory," Bell system technical journal, vol. 11, no. 1, pp. 126$147,1932$. 
[68] H. W. Bode, Network Analysis and Feedback Amplifier Design. V Van Nostrand, 1945.

[69] K. G. Voyce and J. H. McCandless, "Power amplifier linearization using IF feedback," in Microwave Symposium Digest, 1989., IEEE MTT-S International. IEEE, 1989, pp. $863-866$.

[70] V. Petrovic and W. Gosling, "Polar-Loop Transmitter," IEE Electronic Letters, pp. 286-287, vol. 15, No. 10, May 1979.

[71] V. Petrovic, "VHF SSB Transmitter employing Cartesian Feedback," in Proceedings of IEE Conference on Radio and Information Theory, May 1984, pp. 161-165.

[72] C.-N. Chen and D. Hoult, Biomedical Magnetic Resonance Technology. Adam Hilger, Bristol, 1989.

[73] R. Middlebrook, "Measurement of loop gain in feedback systems," International Journal of Electronics Theoretical and Experimental, vol. 38, no. 4, pp. 485-512, 1975.

[74] J. Y. Lu, P. P. Stang, J. M. Pauly, M. G. Zanchi, and G. C. Scott, "Analysis of Gain and Noise Relationship in RF Feedback Power Amplifier Linearization for Use at 1.5T MRI," in $22^{\text {nd }}$ Proc. Intl. Soc. MRM, p. 1334, 2014.

[75] K. K. Mohammed and R. B. Mohammed, "Linearization of power amplifier class AB using Cartesian feedback," in Systems Signals and Devices (SSD), 2010 7th International Multi-Conference on. IEEE, 2010, pp. 1-6.

[76] J. L. Dawson and T. H. Lee, "Cartesian feedback for RF power amplifier linearization," in Proceedings of the American Control Conference, vol. 1, 2004, pp. 361-366. 
